\title{
ANALYSIS OF RAD9 FUNCTIONS: ROLES IN THE CHECKPOINT RESPONSE, DNA DAMAGE PROCESSING, AND PREVENTION OF GENOMIC INSTABILITY
}

by Kara Ann Kenady Nyberg

\begin{abstract}
A Dissertation Submitted to the Faculty of the DEPARTMENT OF MOLECULAR AND CELLULAR BIOLOGY
\end{abstract}

In Partial Fulfillment of the Requirements for the Degree of DOCTOR OF PHILOSOPHY

In the Graduate College THE UNIVERSITY OF ARIZONA 
UMI Number: 3089997

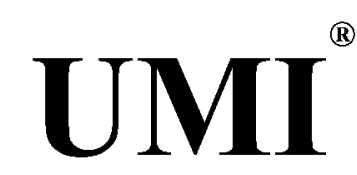

UMI Microform 3089997

Copyright 2003 by ProQuest Information and Learning Company.

All rights reserved. This microform edition is protected against unauthorized copying under Title 17, United States Code.

ProQuest Information and Learning Company 300 North Zeeb Road

P.O. Box 1346

Ann Arbor, MI 48106-1346 
THE UNIVERSITY OF ARIZONA $\circledast$ GRADUATE COLLEGE

As members of the Final Examination Committee, we certify that we have read the dissertation prepared by Kara Ann Kenady Nyberg.

entitled RNALYSIS OF RAD9 FUNCTIONS: ROLES IN THE CHECKPOINT RESPONSE DNA DAMAGE PROCESSING AND PREVENTION OF GENOMIC INSTABILITY

and recommend that it be accepted as fulfilling the dissertation requirement for the Degree of Doctor of Philosophy
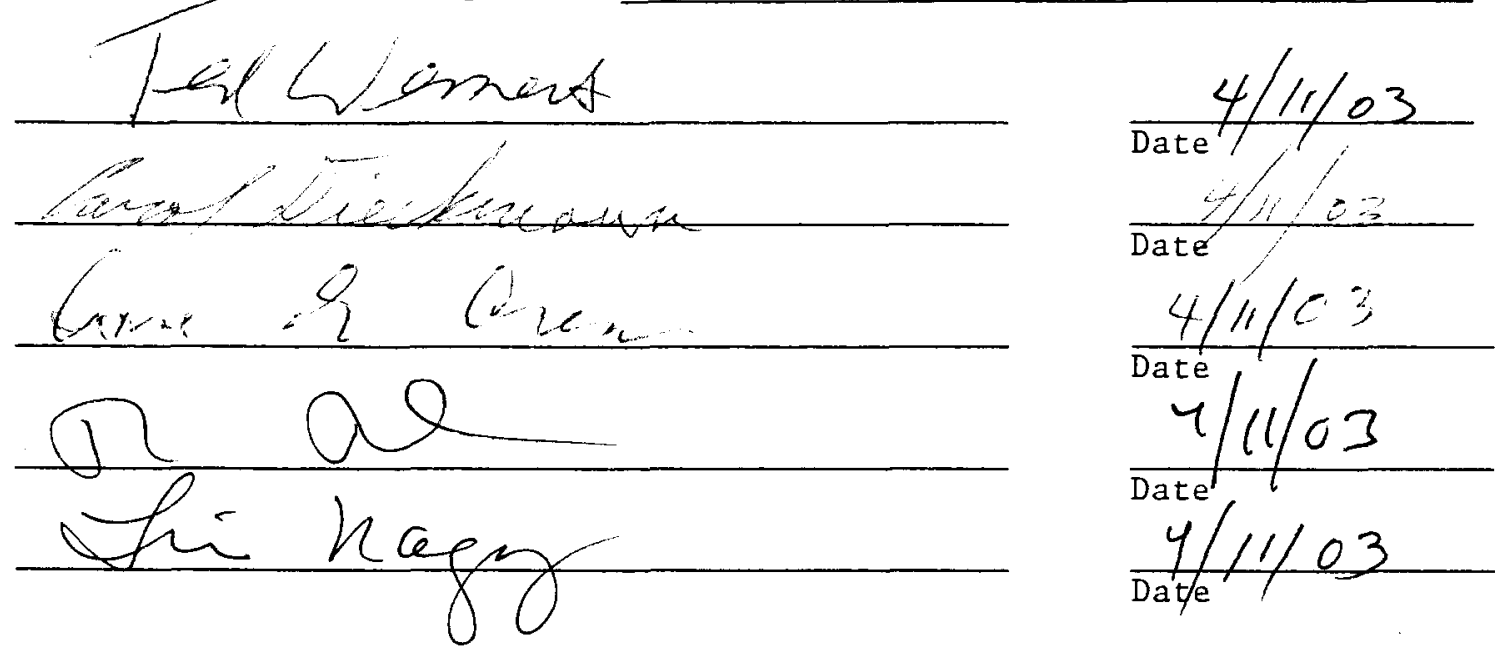

Final approval and acceptance of this dissertation is contingent upon the candidate's submission of the final copy of the dissertation to the Graduate College.

I hereby certify that I have read this dissertation prepared under my direction and recommend that it be accepted as fulfilling the dissertation requirement.
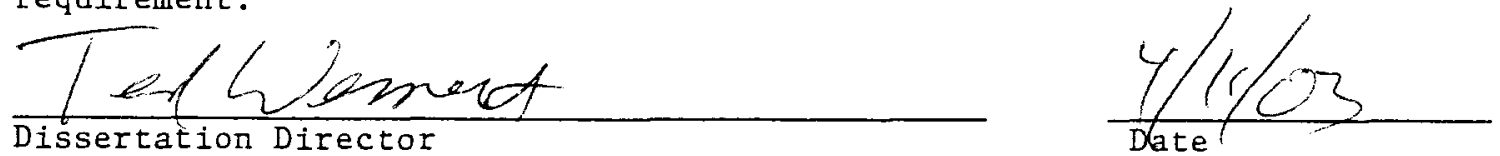


\section{STATEMENT BY AUTHOR}

This dissertation has been submitted in partial fulfillment of requirements for an advanced degree at the University of Arizona and is deposited in the University Library to be made available to borrowers under rules of the Library.

Brief quotations from this dissertation are allowable without special permission, provided that accurate acknowledgement of source is made. Requests for permission for extended quotation from or reproduction of this manuscript in whole or in part may be granted by the head of the major department or the Dean of the Graduate College when in his or her judgment the proposed use of the material is in the interests of scholarship. In all other instances, however, permission must be obtained from the author.

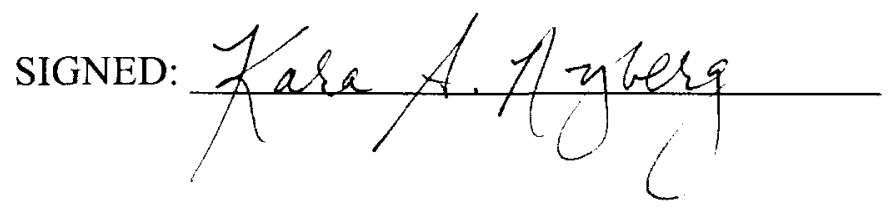




\section{ACKNOWLEDGEMENTS}

I owe great thanks to many people who were instrumental in helping me to succeed and perservere through graduate school.

First and foremost, I thank my graduate advisor, Ted Weinert. My first experience with Ted occurred when I was a senior in college. Ted came and spoke as a guest lecturer for the Genetic Analysis course I was taking. I remember being throroughly impressed by the excitement and passion he showed for his research on the DNA damage checkpoint pathway. That exuberance eventually led me to the University of Arizona and specifically to Ted's lab. For all the guidance, support, and undying enthusiasm for my science, I owe Ted endless thanks.

I would next like to acknowledge my graduate committee - including Roy Parker, Lisa Nagy, Anne Cress, and Carol Dieckmann - for being always insightful, always positive, and for always challenging me to the utmost of my abilities. Roy deserves much extra thanks, as he helped to co-mentor me throughout my graduate career. I have never before had such an incredible, influential mentor, and I feel tremendously fortunate that he helped to guide me throughout my stay here.

I owe much thanks to all my fellow laboratory members, present and past, including, Rhett Michelson, Charley Putnam, Saul Rosenstein, Nicole Danzl, Lisa Shanks, Elizabeth Little, and Parma Sundareshan. To Rhett, who I regard as a big brother, and Charley, who I regard as a father figure, I will miss you both dearly but always keep you close in my heart.

I would like to thank other labs for the advice and support they offered, especially the Parker and Vierling labs, and the entire Moelcular and Cellular Biology Deparmtent.

I owe Kenny Friedrich many thanks for helping me to laugh my way through some of the roughest parts of graduate school. He serves as an inspiration when trying to fight one's way out of the quagmire of pessimism.

Finally, I owe a million thanks to my family, especially my mother, for supporting me throughout my stay here.

This work was supported by a UA Cancer Biology Training Grant through NIH and a Breast Cancer Research and Training Grant from the Department of Defense (DAMD1701-1-0127). 


\section{DEDICATION}

My dissertation is dedicated to all of my family: my mother, Lawrence, my father, Jana, Scott, Bobby, Nana, Poppy, Grandma Gladys, Grandma Ella, Cotty, and Matie.

For always supporting me, encouraging me, and believing in me, I owe you all countless thanks. 
TABLE OF CONTENTS

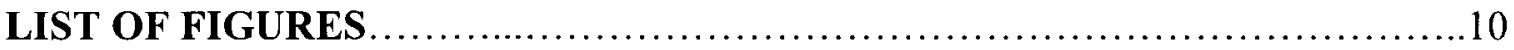

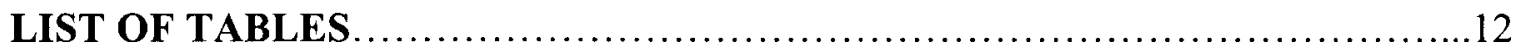

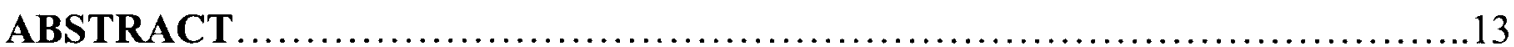

CHAPTER 1. TOWARD MAINTAINING THE GENOME: DNA DAMAGE AND

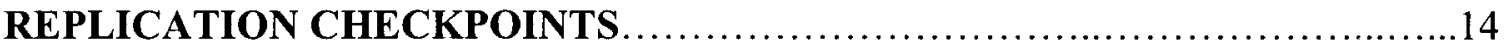

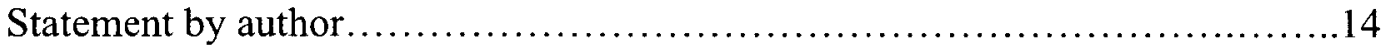

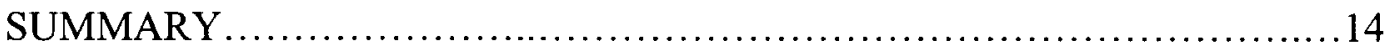

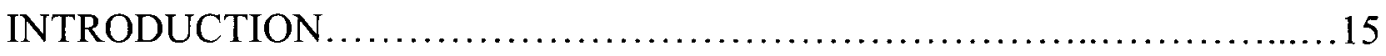

THE CHECKPOINT PROTEINS ......................................... 19

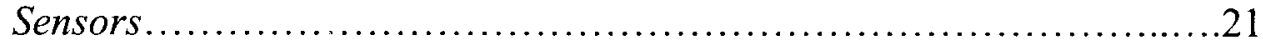

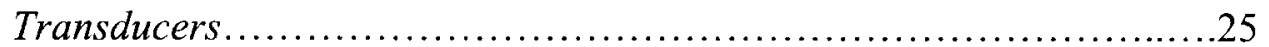

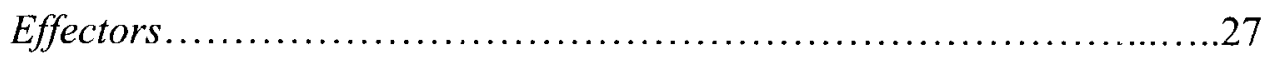

The Molecular Signals that Activate Checkpoint Responses..................27

The Molecular Pathways Associated with DNA Damage.........................31

ATR and Single-Strand DNA Response Complexes....................32

ATM and Double-Strand Break Response Complexes...................33

The Molecular Pathways Associated with Replication Forks.....................35

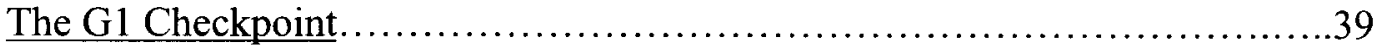

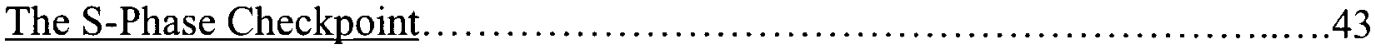

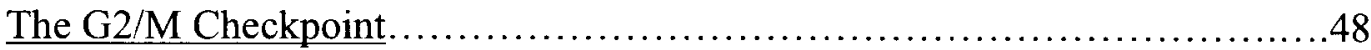

Damage Repair and Recovery .....................................5 54

DNA Checkpoints and Their Link to Repair.......................54

Recovery, Adaptation, and Apoptosis..............................58

Additional Roles of Checkpoint Proteins in DNA Metabolism.................60

Concluding Remarks Regarding Genome Stability.........................63

CHAPTER 2. ANALYSIS OF RAD9 FUNCTIONS BY IDENTIFYING

SEPARATION-OF-FUNCTION MUTATIONS .............................. 
TABLE OF CONTENTS - Continued

Statement by author....................................................66

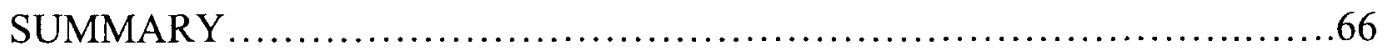

INTRODUCTION ...................................................67

The opposing role of checkpoint proteins in ssDNA formation...........71

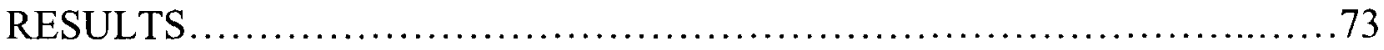

Creation of rad9 separation-of-function mutants.....................73

Screening rad 9 separation-of-function mutants.......................75

Quantitative analysis of putative separation-of-function mutants..........78

Determining the role of $M E C 1, R A D 53, D U N 1$, and $C H K 1$ in the inhibition-of-degradation phenotype $. . \ldots \ldots \ldots \ldots \ldots \ldots \ldots \ldots . \ldots . \ldots . \ldots 5$

Creation of $\operatorname{rad} 9$ point mutants not putatively recognized by Rad53 ....89

Direct detection of ssDNA formation in rad9-Y829F $F \ldots \ldots \ldots \ldots \ldots . . . \ldots 9$

Attempts to create stronger rad9 separation-of-function alleles..........95

Additional efforts to determine if arrest plays a role in attenuating an inhibition-of-degradation defect $\ldots \ldots \ldots \ldots \ldots \ldots \ldots \ldots \ldots \ldots . . . \ldots 6$

Determining the role of TOF1 in the inhibition-of-degradation phenotype........................................... 100

The role of $A S F 1$ in inhibiting DNA degradation................... 102

Experiments that would confirm and further test Asfl's role in the inhibition-of-degradation function...$\ldots \ldots \ldots \ldots \ldots \ldots \ldots \ldots \ldots$

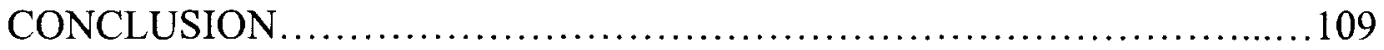

\section{CHAPTER 3. THE BRCT REPEATS OF THE RAD9 CHECKPOINT PROTEIN}

ARE PREDOMINANTLY REQUIRED FOR CONCENTRATING RAD9........112

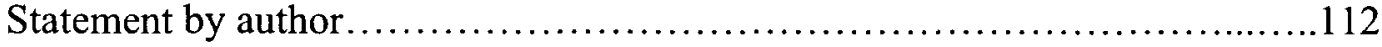

SUMMARY ........................................................... 112

INTRODUCTION ................................................ 114

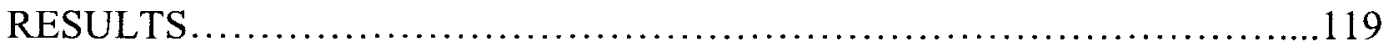

Expression of rad9 $\triangle \mathrm{BRCT}$ s results in DNA damage sensitivity.........119 


\section{TABLE OF CONTENTS - Continued}

Overexpression of rad9BRCTs confers DNA damage resistance. 120

Overexpression of the BRCTs alone does not cause a dominant-negative

effect.

Overexpression of $\operatorname{Rad} 9$ or $\operatorname{rad} 9 \Delta \mathrm{BRCT}$ s does not cause constitutive arrest.

Restoration of wild-type function of $\operatorname{rad} 9 \Delta B R C T$ s by forced dimerization.

Direct tests to confirm rad9 $\triangle B R C T s-G S T$ constitutively dimerizes.

Determining if Rad9 constitutively dimerizes irrespective of DNA damage.

Activation of downstream kinases by rad9 $\triangle B R C T s-G S T$.

$\operatorname{rad} 9 \triangle B R C T_{s}-G S T$ demonstrates cell cycle arrest kinetics identical to that of $\operatorname{Rad} 9$.

$\underline{\operatorname{rad} 9 \triangle \mathrm{BRCTs}-\mathrm{GST} \text { causes the same adaptation response as that of }}$ $\underline{\operatorname{Rad} 9}$.

The role of Rad 9 and $\operatorname{rad} 9 \triangle B R C T$ s in response to S-phase damage.

Analyzing $R A D 9 \mathrm{BRCT}$ point mutations and the effects on protein function. 138

The $\operatorname{rad} 9^{\mathrm{MutF}}, \operatorname{rad} 9^{\mathrm{MutW}}$, and $\operatorname{rad} 9^{\mathrm{MutF}+\mathrm{W}}$ proteins are all completely defective for S-phase functions.

Determining if the null phenotype of $\operatorname{rad} 9^{\mathrm{MutF}+\mathrm{W}}$ can be explained by protein localization defects................................143

Forcing aberrant Rad9 associations does not affect function. 143

Does unphosphorylatable rad9 still dimerize? 145 CONCLUSIONS. 


\section{TABLE OF CONTENTS - Continued}

$\underline{\operatorname{Rad} 9 \text { 's BRCT domains predominantly function to concentrate }}$

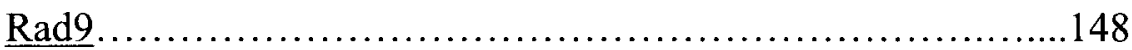

Other possible roles mediated by Rad9's BRCT domains...............149

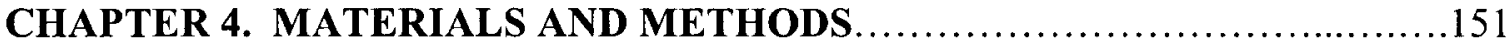

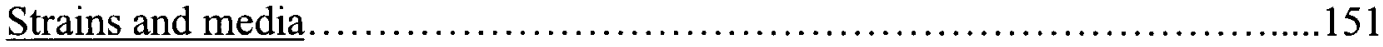

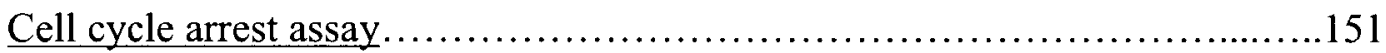

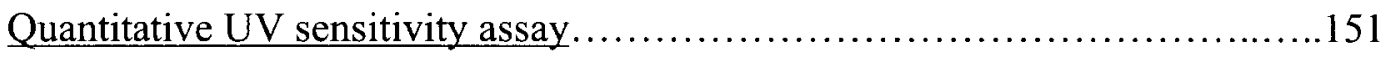

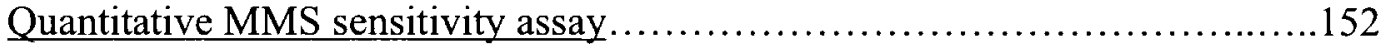

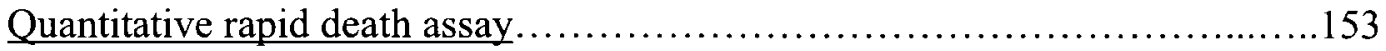

DNA purification for Quantitative Amplification of ssDNA

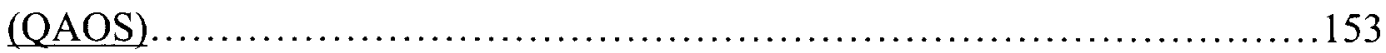

Site-directed mutagenesis............................................. 155

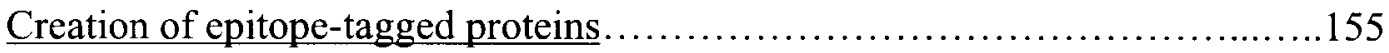

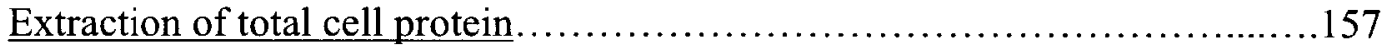

Immunoprecipitation of cell protein .................................. 157

Western blot transfer and protein detection.................................. 159

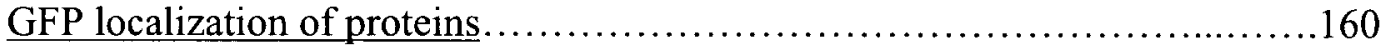

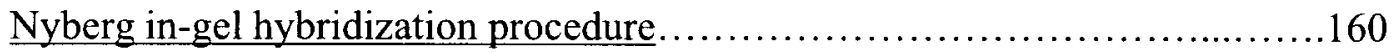

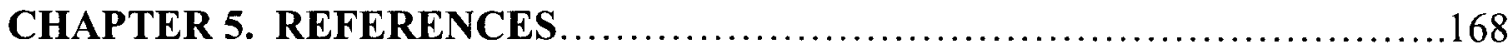




\section{LIST OF FIGURES}

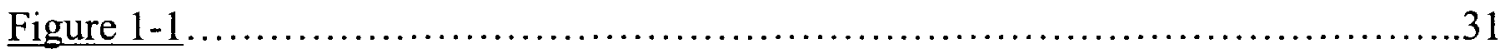

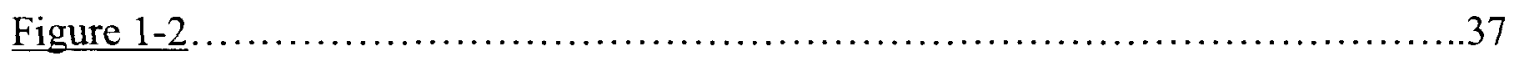

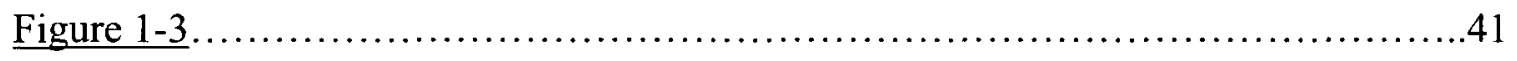

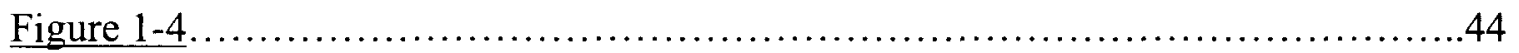

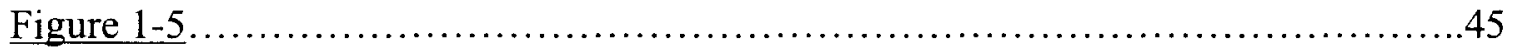

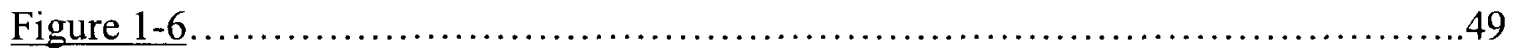

Figure 2-1. A current model of the checkpoint cascade leading to $\mathrm{G}_{2} / \mathrm{M}$ arrest..........69

Figure 2-2. The pentapeptide insertional mutagenesis scheme $\ldots \ldots \ldots \ldots \ldots \ldots \ldots \ldots .74$

Figure 2-3. Phenotypic analysis of putative rad9 separation-of-function mutants in

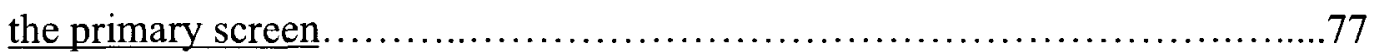

Figure 2-4. Comparison of rad9 3-E5 and rad9 16-C5 phenotypes..................81

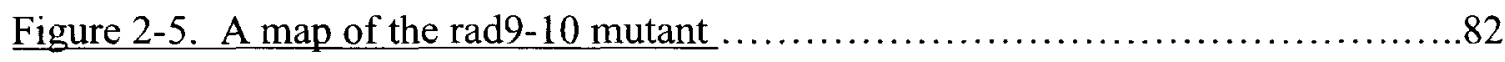

Figure 2-6. UV and MMS sensitivities of rad9-10 assayed at $30^{\circ} \mathrm{C} \ldots \ldots \ldots \ldots \ldots \ldots . \ldots 4$

Figure 2-7. Artificial arrest attenuates the inhibition-of-degradation defect.............86

Figure 2-8. Inhibition-of-degradation phenotypes of mecl smll, rad53-1, dunl, and chkl

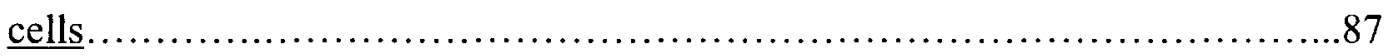

Figure 2-9. Phenotypic analysis of the rad $9-Y 829 \mathrm{~F}$ allele $\ldots \ldots \ldots \ldots \ldots \ldots \ldots \ldots . . \ldots 2$

Figure 2-10. Generation of ssDNA in the rad9-Y829F strain........................94

Figure 2-11. A rad9-Y829F mecl smll strain does not show an appreciable increase in defective inhibtion-of-degradation function...............................96

Figure 2-12. Kinetics of cell cycle progression after damage-induced arrest in rad9-10

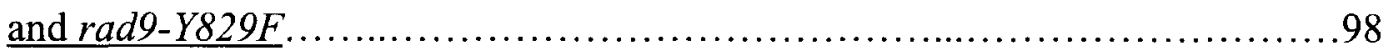

Figure 2-13. The doubly-mutated rad9-10, Y829F allele has the same inhibition-ofdegradation defect as each single mutant alone............................ 100

Figure 2-14. tofl mutation does not create a synergistic defect in rad 9 inhibition-of-

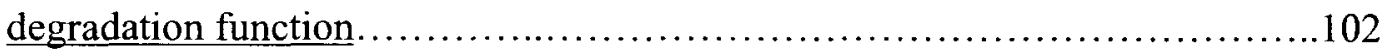




\section{LIST OF FIGURES - Continued}

Figure 2-15. A $c d c 13-1$ asfl mutant shows an inhibition-of-degradation

defect. 105

Figure 2-16. Asf1-GFP foci formation after $c d c 13-1$ damage induction. 108

Figure 3-1. A map of the Rad9 protein and phenotypic effect of deleting the BRCT domains. 120

Figure 3-2. Overexpression levels of the $\operatorname{rad} 9 \Delta B R C T$ s protein and phenotypic analysis.

Figure 3-3. Characterization of strains containing non-dimerizing $\operatorname{rad} 9 \triangle \mathrm{BRCTs}-\mathrm{Myc}$ and dimerizing rad9 $\triangle B R C T s-G S T$ proteins 125

Figure 3-4. Co-immunoprecipitation of epitope-tagged $\operatorname{Rad} 9$ and $\operatorname{rad} 9 \triangle \mathrm{BRCTs}$ proteins. 128

Figure 3-5. Co-immunoprecipitation of epitope-tagged $\operatorname{Rad} 9$ in a strain containing CDC13. 130

Figure 3-6. Activation of downstream kinases by dimerizing rad9 $\triangle B R C T s-G S T$ but not non-dimerizing $\operatorname{rad} 9 \Delta \mathrm{BRCTs}-\mathrm{Myc}$. .131

Figure 3-7. S-phase responses of cells containing non-dimerizing $\operatorname{rad} 9 \Delta B R C T s-M y c$ and

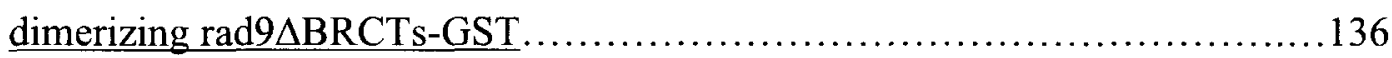

Figure 3-8. Phenotypic and protein characterization of cells containing $\operatorname{rad} 9^{\mathrm{MutF}+\mathrm{W}}$ and

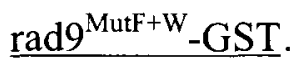

Figure 4-1. Nyberg in-gel hybridization showing ssDNA accumulation in $\mathrm{rad}^{-}$versus $\underline{R A D 9}$ strains 162 


\section{LIST OF TABLES}

Table 1-1. Orthologous checkpoint proteins ...................................20

Table 2-1. Comparison of phenotypes of putative separation-of-function mutants......79

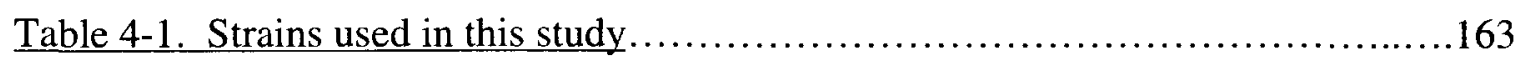

Table 4-2. Pringle pimer pairs for C-terminally tagging $R A D 9, A S F 1$, and $D D C 1 \ldots \ldots 156$ 


\begin{abstract}
In the 15 years since Rad9's discovery, we have come to understand a great about Rad9 biology, yet numerous questions still remain. Especially intriguing questions include: a) How does Rad9 get localized and/or recognize DNA damage? b) How does Rad9 activate downstream checkpoint proteins? and c) Does Rad9 play additional roles in recognizing and/or repairing DNA damage that have yet to be discovered?

In an effort to try to answer some of these questions, I analyzed the effects of various $R A D 9$ mutations. To assess the contribution of RAD9 to inhibiting DNA degradation and its role in the cell cycle arrest and DNA damage repair responses, I performed a pentapeptide mutagenesis screen in order to obtain $R A D 9$ separation-offunction mutants that were proficient for one known phenotype and deficient in the other. I was able to obtain 2 such mutants that were hypomorphic in their ability to prevent DNA degradation and completely proficient for arresting the cell cycle in the presence of DNA damage and for repairing such damage. Despite many efforts, I was unable to enhance the hypomorphic dysfuntion of these mutants in preventing DNA degradation such that a suppressor screen to identify other genes in this pathway could be performed. In another effort to try to understand the role of the BRCT domains for RAD 9 functions, I analyzed the effects of other various $R A D 9$ mutants. By deletion analysis, I was able to determine that the predominant function of RAD9's BRCT domains is to mediate concentration of the Rad9 protein for function by two means: 1) by conferring stability to the Rad9 protein, and 2) by homodimerizing Rad9 to increase its local concentration to enable interactions with downstream checkpoint components.
\end{abstract}




\section{CHAPTER 1. TOWARD MAINTAINING THE GENOME: DNA DAMAGE AND REPLICATION CHECKPOINTS}

Statement by author

The following is a review that I wrote with additional help from Ted Weinert, Rhett Michelson, and Charles Putnam. I did the vast majority of the research and writing. Weinert and I had numerous conversations that were critical for development of many of the points within the review, and he was extremely influential in the editing process. Michelson mainly contributed all of the figures, while adding some additional insights, and Putman assisted in collecting background information and editing.

\section{SUMMARY}

DNA checkpoints play a significant role in cancer pathology, perhaps most notably in maintaining genome stability. This review summarizes the genetic and molecular mechanisms of checkpoint activation in response to DNA damage. The major checkpoint proteins common to all eukaryotes are identified and discussed, together with how the checkpoint proteins interact to induce arrest within each cell cycle phase. Also discussed are the molecular signals that activate checkpoint responses, including singlestrand DNA, double-strand breaks, and aberrant replication forks. We address the connection between checkpoint proteins and damage repair mechanisms, how cells recover from an arrest response, and additional roles that checkpoint proteins play in DNA metabolism. Finally, the connection between checkpoint gene mutation and genomic instability is considered. 


\section{INTRODUCTION}

Growth and division of a single cell to yield two daughter cells requires the coordination of numerous events, in particular the faithful replication and partitioning of the cell's genetic material to each daughter cell. At the extreme, errors in this process could mean death for a unicellular organism, and for multicellular organisms, faulty cell division may ultimately culminate in developmental defects or oncogenesis.

To ensure the fidelity of division, cells have evolved general mechanisms called checkpoints that monitor the successful completion of cell cycle events. Checkpoints are typically not essential for cell cycle events per se, but rather they make certain that events are completed correctly and in the proper order. When one cell cycle event has not been successfully completed, checkpoints will delay progression until the step is correctly accomplished, and only then will they relieve the arrest to allow the cell to move onto the next phase.

Given that cells are constantly under the assault of endogenous and exogenous forms of damage, maintaining a complete, undamaged genome is a continual challenge and of vital importance to the cell and future cellular generations. All eukaryotic cells, except certain embryonic cells, possess checkpoints to monitor virtually every cell cycle event involving DNA metabolism (Hartwell \& Weinert, 1989). Cells not only supervise the process of DNA replication during S-phase to ensure the correct completion of this 
event, but they also monitor the state of DNA throughout the entire cell cycle to minimize the accruement of damage. In addition to arresting cells with compromised DNA, these checkpoints also mediate repair of the damage, a role still being elucidated. Therefore, these DNA checkpoints - or, perhaps more specifically, DNA maintenance checkpoints include what may have traditionally been thought of as two classes: $(a)$ the DNA damage checkpoints that recognize and respond to DNA damage, and $(b)$ the replication checkpoints that monitor the fidelity of copying DNA.

The importance of DNA checkpoints in human pathology, and especially cancer, is now well established. The p53 tumor suppressor, the most infamous of checkpoint genes, is the only one routinely mutated in sporadic human cancers (reviewed by Bargonetti \& Manfredi, 2002). Although p53's role in G1 arrest was first described 11 years ago (Kastan et al., 1991), our understanding of its function is incomplete. Early hypotheses that arrest provides time for DNA repair whereas apoptosis eliminates cells with irreparable damage from cycling proves insufficient. In addition, how each p53 function - G1 arrest, G2 arrest, and apoptosis - relates to human cancer awaits the description of molecular mechanisms in specific cellular contexts.

Other human disease genes have links to cancer pathology, although again those links are unresolved. ATM (ataxia telangiectasia mutated) is a protein kinase central to all DNA maintenance responses that, when absent, results in the disease ataxia telangiectasia (AT). AT is characterized at the cellular level by gross chromosomal 
rearrangements and radioresistant DNA synthesis, and at the organismal level by immune deficiency, cerebellar degeneration, and an increased predisposition to cancer (Savitsky et al., 1995; Lavin \& Shiloh, 1997). Not known is whether the disease is due to defects in DNA damage responses or to defects in some other cellular activity regulated by ATM. Humans harboring mutations in proteins that have recently been shown to interact with ATM in DNA damage recognition, Mre 11 and Nbsl, also present with AT-like symptoms, thereby suggesting a connection to checkpoint and DNA repair defects (Carney et al., 1998; Stewart et al., 1999). Additional links include mutation of either Chk2 or p53, both of which act downstream of ATM, resulting in a genetic disease called Li Fraumeni syndrome that dramatically predisposes patients to cancer development (Malkin et al., 1990; Bell et al., 1999). Mutations in the checkpoint protein BRCA1, another substrate of ATM, result in defects in S-phase and G2 arrest (Xu et al., 2001) and also cause defects in DNA repair (Abbott et al., 1999; Moynahan et al., 1999; Bhattacharyya et al., 2000). Notably, an estimated $50 \%$ of inherited cases of breast cancer are likely due to mutations in the $B R C A 1$ gene, and $B R C A 1$ mutation is implicated in almost all families with histories of both ovarian and breast cancer (Gayther et al., 1998). Such results clearly demonstrate that DNA checkpoints play a central role in human pathology, and we now must face the challenge of understanding the molecular intricacies as to how and why.

In the past few years, studies in organisms from budding and fission yeasts, the fungus Aspergillus, nematodes, Drosophila, mouse, to mammalian systems have 
combined to enhance our understanding of these DNA maintenance checkpoints in cell cycle biology. The recurrent theme is the importance of these checkpoints in maintaining genome stability and the correlation of loss of this function with the human pathology of cancer. Cells lacking functional checkpoints display genomic instability due to a failure to properly respond to DNA damage, faulty DNA replication, or aberrant chromosome segregation, resulting in an accelerated mutator phenotype (Loeb, 1991). Recent understanding of molecular mechanisms summarized in this review may provide some insight into the causes of genomic instability and suggest strategies for development of therapeutics. Of promise, researchers hope to exploit checkpoint defects in cancer cells to selectively kill them.

This review describes our current understanding of the molecular and genetic pathways of the DNA damage and replication checkpoints. We present working models based on available data from studies of various organisms, most notably those performed in mammalian cells and budding and fission yeast, and point out that many molecular and biochemical details needed to support some of the proposed models are lacking. Checkpoint controls in different organisms indicate a high degree of conservation, although occasional divergences do occur. 


\section{THE CHECKPOINT PROTEINS}

The checkpoint pathways involve three major groups of proteins that act in concert to translate the signal of damaged DNA into the response of cell cycle arrest and repair. These groups include (a) sensor proteins that recognize damaged DNA directly or indirectly and function to signal the presence of abnormalities, initiating a biochemical cascade of activity; (b) transducer proteins, typically protein kinases, that relay and amplify the damage signal from the sensors by phosphorylating other kinases or downstream target proteins; and (c) effector proteins, which include the most downstream targets of the transducer protein kinases, and are thus regulated, usually by phosphorylation, to prevent cell cycle progression. Table 1 summarizes the principal orthologous checkpoint proteins identified thus far in mammals and budding and fission yeasts. Within the text, the mammalian nomenclature is used unless otherwise stated. Below, we name and briefly discuss the primary proteins involved in DNA checkpoint responses. More detailed discussion of their roles follows in subsequent sections. 
Table 1. Orthologous Checkpoint Proteins.

\begin{tabular}{|c|c|c|c|}
\hline Protein function & Mammals & S. pombe & S. cerevisiae \\
\hline \multicolumn{4}{|l|}{ Sensors } \\
\hline RFCl-like & $\operatorname{Rad} 17$ & $\operatorname{Rad} 17$ & $\operatorname{Rad} 24$ \\
\hline \multirow[t]{3}{*}{ PCNA-like } & $\operatorname{Rad} 9$ & $\operatorname{Rad} 9$ & Ddc1 \\
\hline & Rad 1 & Rad1 & $\operatorname{Rad} 17$ \\
\hline & Hus1 & Hus1 & Mec3 \\
\hline \multirow[t]{2}{*}{ BRCT-contain } & BRCA1 & Crb2/Rph9 & $\operatorname{Rad} 9$ \\
\hline & TopBP1 & Cut5 & Dpb11 \\
\hline \multirow[t]{3}{*}{ DSB recognition/repair } & Mre11 & Rad32 & Mrel1 \\
\hline & $\operatorname{Rad} 50$ & Rad50 & $\operatorname{Rad} 50$ \\
\hline & Nbs 1 & & Xrs2 \\
\hline \multicolumn{4}{|l|}{ Replication proteins } \\
\hline DNA polymerase & Pol2 & $\mathrm{Cdc} 20$ & Pol2 \\
\hline DNA helicase & BLM, WRN & Rhq1/Rad12 & Sgs1 \\
\hline Topoisomerase & Top3 & Top3 & Top3 \\
\hline clamp loader & $\mathrm{Rfc} 2-5$ & Rfc2-5 & Rfc2-5 \\
\hline binds ssDNA & Rpa2 & & $\mathrm{Rfa} 2$ \\
\hline \multicolumn{4}{|l|}{ Transducers } \\
\hline \multirow{2}{*}{$\begin{array}{l}\text { PI3-kinases } \\
\text { (PIKK) }\end{array}$} & ATR & $\operatorname{Rad} 3$ & Mecl \\
\hline & ATM & Tell & Tell \\
\hline PIKK binding partner & ATRIP & $\operatorname{Rad} 26$ & $\operatorname{Ddc} 2 / \mathrm{Lcd} 1$ \\
\hline \multirow[t]{2}{*}{ Effector Kinases } & Chk1 & Chkl & Chkl \\
\hline & Chk2 & Cdsl & $\operatorname{Rad} 53$ \\
\hline Replication fork & - & -- & Tof1 \\
\hline stabilizers & -- & Mrcl & Mrcl \\
\hline
\end{tabular}


Sensors.

Proteins have been placed in the sensor class based on genetic and biochemical findings or inference, as little direct biochemical evidence yet exists. Some sensors are thought to directly associate with damaged DNA; others are most likely indirectly associated by interactions with the aforementioned sensors. The types of sensor proteins that associate with a particular DNA lesion may serve as recognition complexes to modulate, recruit, and localize specific target proteins for that lesion type.

\section{$\underline{\operatorname{Rad} 17-R F C \text { and 9-1-1. }}$}

These two complexes of proteins are presumed to act in concert with one another and have functional analogs in DNA replication. As first shown in budding and fission yeasts, $\operatorname{Rad} 17$ interacts with four replication factor $C$ subunits $(R f c 2, R f c 3, R f c 4, R f c 5)$ to form a pentameric structure, referred to as the Rad17- RFC complex (Griffiths et al., 1995; Green et al, 2000; Lindsey-Boltz et al., 2001). Another complex is a heterotrimeric ring composed of Rad9, Hus1, and Rad1, termed the 9-1-1 complex (St. Onge et al., 1999; Volkmer \& Karnitz, 1999; Caspari et al., 2000; Hang \& Lieberman, 2000; Venclovas \& Thelen, 2000; Burtelow et al., 2001). The RFC subunits found in Rad17RFC are more commonly recognized for their association with $\mathrm{Rfc} 1$ during replication to form what is termed the "clamp loader". The replication RFC clamp loader complex recognizes single-strand/double-strand DNA junctions and loads the homotrimeric PCNA "sliding clamp" complex, which encircles the DNA to act as a general scaffold upon which DNA polymerases and other DNA replication proteins are assembled (Tsurimoto 
\& Stillman, 1991). One infers from sequence and structural similarities that during a checkpoint response the Rad17-RFC complex may recognize damage and load the 9-1-1 sliding clamp onto DNA. Indeed, association of Rad17-RFC and the 9-1-1 heterotrimer have been identified in vitro, and each is inferred to bind to sites of DNA damage in vivo as the Rad17-RFC complex binds preferentially to DNA with primer-template-like structures (Lindsey-Boltz et al., 2001). The 9-1-1 complex may have an additional role of processing DNA damage to generate more single-strand DNA, perhaps required for the 9-1-1 complex to better bind DNA or to enhance damage signaling to other checkpoint components (Lydall \& Weinert, 1995; Parker et al., 1998). While the two human Rad17-RFC and 9-1-1 complexes have been reconstituted individually in vitro from recombinant proteins, loading of the 9-1-1 complex onto DNA by Rad17-RFC has not yet been shown. A recent report, however, does indicate that mammalian Rad17 acts to recruit the 9-1-1 complex to sites of damage in vivo just as in yeast, though the consequences of this association have yet to be elucidated (Zou et al., 2002).

\section{BRCA1.}

BRCA1 appears to function as an adaptor of checkpoint initiation by localizing additional substrates for transducer kinase phosphorylation and by perhaps linking checkpoint arrest to DNA damage repair. BRCA1 co-localizes with a number of proteins involved in DNA repair and/or replication, including Rad51, PCNA, Mrel1-Rad50-Nbs1, histone deactelyases, the DNA helicase BLM, and mismatch repair proteins (Scully et al., 1997; Yarden \& Brody, 1999; Zhong et al., 1999; Wang et al., 2000). Additionally, it has 
been shown to directly bind non-specific DNA in vitro (Paull et al., 2001). Consistent with these associations, BRCAl affects homologous recombination, mismatch repair, and transcription-coupled repair (Gowen et al, 1998; Abbott et al., 1999; Moynahan et al., 1999; Snouwaert et al., 1999; Wang et al., 2000; Wang et al., 2001).

The question of whether BRCA1 has fission and budding yeast orthologs (Crb2 and $\operatorname{Rad} 9$, respectively) remains speculative. Based on functional and sequence similarities, however, we suggest that $\mathrm{Crb} 2$ and $\operatorname{Rad} 9$ should be considered BRCA1 orthologs. All three proteins share limited sequence similarity in that each contains two BRCT (Brcal Carboxy Terminal) domains - motifs most likely involved in dimerization (reviewed in Huyton et al., 2000). In addition, their regulation is similar: BRCA1, Crb2, and Rad9 are all phosphorylated after damage by ATR and ATM orthologs (Saka et al., 1997; Emili, 1998; Vialard et al., 1998; Cortez et al., 1999; Tibbetts et al., 2000), and each is required to activate downstream protein kinases (Saka et al., 1997; Grenon et al., 1999; Sanchez et al., 1999; Lee et al., 2000; Gilbert et al., 2001; Yarden et al., 2002). Furthermore, each acts to induce a G2/M cell cycle arrest to allow time for DNA repair (Weinert \& Hartwell, 1988; Saka et al., 1997; Willson et al., 1997; Xu et al., 2001). Differences in other protein functions are expected, given the different nature of the organisms, but the functional core similarities listed here warrants their being termed orthologs. 


\section{Mre11-Rad50-Nbs1.}

The Mre11-Rad50-Nbs1 (MRN) complex localizes to sites of double-strand breaks (DSBs) in vivo and plays vital roles in DNA metabolism, including DSB repair, meiotic recombination, and telomere maintenance (Dolganov et al., 1996; Maser et al., 1997; Chamankhah \& Xiao, 1999; Zhu et al., 2000). Cells deficient for Mrel1 or Nbsl continue DNA replication after damage, known as radioresistant DNA synthesis (RDS), indicating defective checkpoint signaling during S-phase (Shiloh, 1997). Mutations in either gene are now known to affect checkpoints at all phases of the cell cycle (Yamazaki et al., 1998; Buscemi et al., 2001).

\section{Replication proteins.}

We have grouped these proteins because they are all localized at sites of replication forks, play roles in DNA replication, and have checkpoint defects within Sphase. This group includes budding yeast topoisomerase III (Top3; Chakraverty et al., 2001), the Pol2 subunit of the DNA polymerase $\varepsilon$ from budding yeast (Navas et al., 1996), the mammalian DNA helicase BLM and orthologous proteins (Davey et al., 1998;

Frei \& Gasser, 2000; Frei \& Gasser, 2000; Dutertre et al., 2002), the mammalian polymerase recruiter TopBP1 and orthologous proteins (Araki et al., 1995; Verkade \& O'Connell, 1998; Makiniemi et al., 2001; Yamane et al., 2002), eukaryotic members of the RFC clamp loader complex (Rfc2-5; Noskov et al., 1998; Shimada et al., 1999; Naiki et al., 2000; Kim \& Brill, 2001), and the Rpa2 subunit of the single-strand binding 
protein RPA in mammals and budding yeast (Santocanale et al., 1995; Wang et al., 2001).

Transducers.

Transducers include the protein kinases that, when activated by the presence of DNA damage, initiate a signal transduction cascade that propagates and amplifies the damage signal to ultimately cause cell cycle arrest.

\section{ATM \& ATR.}

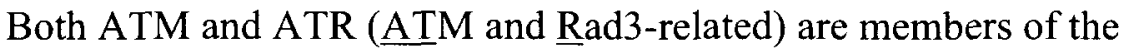
phosphoinositide 3-kinase related kinases (PIKKs) - large proteins ranging between 275 $500 \mathrm{kDa}$ possessing a unique protein kinase domain at their C-termini with little sequence conservation outside this region (Abraham, 2001).

Following observations in yeast, ATR has recently been shown to form a heterodimer with the associated protein ATRIP, both of which are required for the checkpoint signaling pathway (Edwards et al., 1999; Paciotti et al., 2000; Rouse \& Jackson, 2000; Cortez et al., 2001; Wakayama et al., 2001; Wolkow \& Enoch, 2002). The mechanism of ATR-ATRIP activation remains elusive. As ATR is a member of the PIKK family that includes DNA-PK, it is interesting to note that in vitro DNA-PK kinase activity is mostly activated upon association with the Ku heterodimer that binds doublestrand DNA ends (Smith \& Jackson, 1999). Whether ATRIP family members possess 
detectable DNA binding activity or are required for protein kinase activity of ATR is not yet known, though ATR and ATRIP co-localize to intranuclear foci after DNA damage or inhibition of replication (Cortez et al., 2001).

Of interest, ATR in mammalian cells and $\mathrm{Mecl}$ in budding yeast are essential for cell viability, whereas the fission yeast $\operatorname{Rad} 3$ is not. In $S$. cerevisiae, this necessity is due to Mec1's role in regulating dNTP pools (Zhao et al., 2001; Zhao \& Rothstein, 2002). As ATR's essential function has yet to be clearly defined, regulation of dNTP pools in mammalian cells may be an obvious first place to look. Alternately, because mammalian genomes are so large, the DNA damage likely sustained on a continuous basis might need the constant monitoring of the ATR checkpoint to ensure viability. For instance, the Rad51 recombination protein is not essential in yeast but it is in mammalian cells (Tsuzuki et al., 1996).

\section{Chk1 \& Chk2.}

Chk1 and Chk2 are classic serine-threonine kinases that are required for cell cycle arrest in response to DNA damage. As downstream kinases, they are phosphorylated by ATM/ATR-dependent processes, may additionally undergo autophosphorylation (demonstrated for S. cerevisiae Rad53 and Chk1), and serve to potentiate phosphorylation of downstream targets. 
Effectors.

Many targets of transducer phosphorylation have yet to be identified, and those that are known are typically not well conserved across species. Therefore, we will discuss the effects of effector phosphorylation throughout the remaining sections where appropriate.

The Molecular Signals that Activate Checkpoint Responses.

The nature of DNA structures that are recognized by the sensor proteins to activate checkpoint responses remains largely obscure. Even in the well-defined bacterial SOS response system that leads to activation of the RecA protein, defining the lesioned DNA signal in vivo has been a formidable task (Eder et al., 2001). In eukaryotic cells, considering even the simplest hypothesis becomes complex when accounting for the type of damage, the quantity of lesions, and the various protein kinases and substrates involved. Interpreting in vivo studies that utilize irradiation or alkylating agents to induce DNA damage - both of which cause a vigorous checkpoint response - are ambiguous due to the myriad of DNA lesions generated (Ward, 1985). Furthermore, defined biochemical systems that may be used to determine mechanisms of activation in vitro are currently lacking.

Despite these difficulties, a prevailing view for eukaryotic cells is that different kinds of lesions are converted to two common structures, namely single-strand DNA (ssDNA) and double-strand DNA breaks (DSBs), that then signal checkpoints.

Moreover, the weight of evidence suggests that the two signals activate different arms of 
the checkpoint pathways: DSBs activate a pathway containing the ATM kinase family, whereas ssDNA activates a checkpoint pathway containing the ATR kinase family (Figure 2).

Activation of the ATR checkpoint pathway by ssDNA rests on extrapolation from three key observations in budding yeast. In yeast, it is well documented that a single defined DSB generated by the $\mathrm{HO}$ endonuclease is rapidly converted to long tracts $(>5$ $\mathrm{kb}$ ) of ssDNA by degradation of the 5' strand (Sugawara \& Haber, 1992). This ssDNA causes an acute arrest of chromosome segregation that requires the ATR homolog, Mec1, and presumably not the ATM homolog, Tel1 (Sandell \& Zakian 1993). In the second study, a temperature-sensitive $c d c 13$ mutation - a protein normally associated with telomeres that aids in their protection - leads to extensive ssDNA near telomere ends. This defect, too, elicits a profound Mecl-dependent arrest that is Tell independent (Garvik et al., 95; Gardner et al., 1999). Thirdly, unrepaired DSBs (due to mutation of the RecA homolog Dmc1) generated during meiosis undergo 5' end resection to create ssDNA and require Mecl for meiotic arrest (Lydall et al., 1996). The length or quantity of ssDNA required to activate arrest is unknown, as are the proteins needed to associate with ssDNA, though RPA is a plausible candidate (Santocanale et al., 1995; Longhese et al, 1996).

In budding yeast, evidence regarding the activation of Tell by DSBs is less extensive, as these breaks are typically converted rapidly to ssDNA. However, a recent 
study shows activation of Tell using cells where DSB resection is inferred to be blocked in meiosis by rad50S or sae2 mutation (Usui et al., 2001). Meiotic cells with a mec1 mutation show extreme sensitivity to the damaging agent methylmethane sulfonate (MMS). mec1 sae2 or mecl rad50S mutants, however, show suppression of sensitivity, re-establishment of downstream Rad53 phosphorylation, and restoration of cell cycle arrest. These suppressed phenotypes, though, are likewise abolished in a mecl tell sae2 triple mutant. These data support a model whereby suppression of sensitivity in $\mathrm{mecl}$ sae 2 or mec1 rad50S mutants is achieved due to enhanced activation of the parallel checkpoint pathway mediated by Tell that may specifically respond to DSBs. Elimination of both Mecl- and Tell-dependent pathways results in enhanced lethality when DNA damage is incurred (Usui et al., 2001), as presumably both branches of the DNA damage response have been abrogated.

Many other indirect observations support the view that ssDNA activates ATR homologs and DSBs activate ATM-like kinases. $\gamma$-irradiation of mammalian cells, thought to induce DSBs, causes rapid ATM kinase activity, whereas ATR activity increases only at a later time, perhaps in response to DSB processing (Xu \& Baltimore, 1996; Wright et al., 1998). Moreover, immunoprecipitated ATM is more enzymatically active after extraction from cells treated with IR or radiomimetic agents (Banin et al., 1998; Canman et al., 1998). In contrast, disruption of DNA replication in budding and fission yeasts and mammalian cells, thought to generate extensive ssDNA regions, results 
in responses that require activated ATR but not ATM (al-Khodairy et al., 1994;

Paulovich \& Hartwell, 1995; Guo et al., 2000; Hekmat-Nejad et al., 2000).

We think one structure critical for activating checkpoint controls arises from aberrant replication forks. Of principle importance may be the structure and quantity of stalled forks required to signal; whether ssDNA, DSBs, or other structures specific to faulty forks are needed to signal in unknown. A recent electron micrograph study of replication forks isolated from budding yeast provides information for an initial model. Normal replication forks contain approximately 200 base-pairs (bp) of ssDNA (Foiani, submitted). Though yeast contain approximately 332 origins of replication, a normal DNA replication cycle generates about 50 replication forks yet does not appear to activate checkpoint protein kinases (Raghuraman et al., 2001). Stalling of one fork generates $\sim 350$ bp of ssDNA at the fork site, and stalling of 50 replication forks during replication provides a robust activation of protein kinases (Foiani, submitted). Whether it is the amount of ssDNA generated by stalled forks, a stalled fork that collapses to create a DSB or ssDNA, and/or the structure of the stalled replication complex itself that leads to a checkpoint response is unclear. However, just as one DSB is sufficient to cause arrest in yeast cells (Sandell \& Zakian, 1993), we envision that one defective fork is likewise sufficient to signal. Whether a single unperturbed DNA replication fork may also be sufficient to form a signal is unclear (van Brabant et al., 2001). 
The Molecular Pathways Associated with DNA Damage.

Numerous genes and proteins that act in checkpoint pathways have now been identified. We present the general mechanisms for pathways responding to ssDNA and DSBs (Figure 1), recognizing that most molecular aspects rely on inference and are based, in part, on the expectation of functional conservation of orthologous proteins.

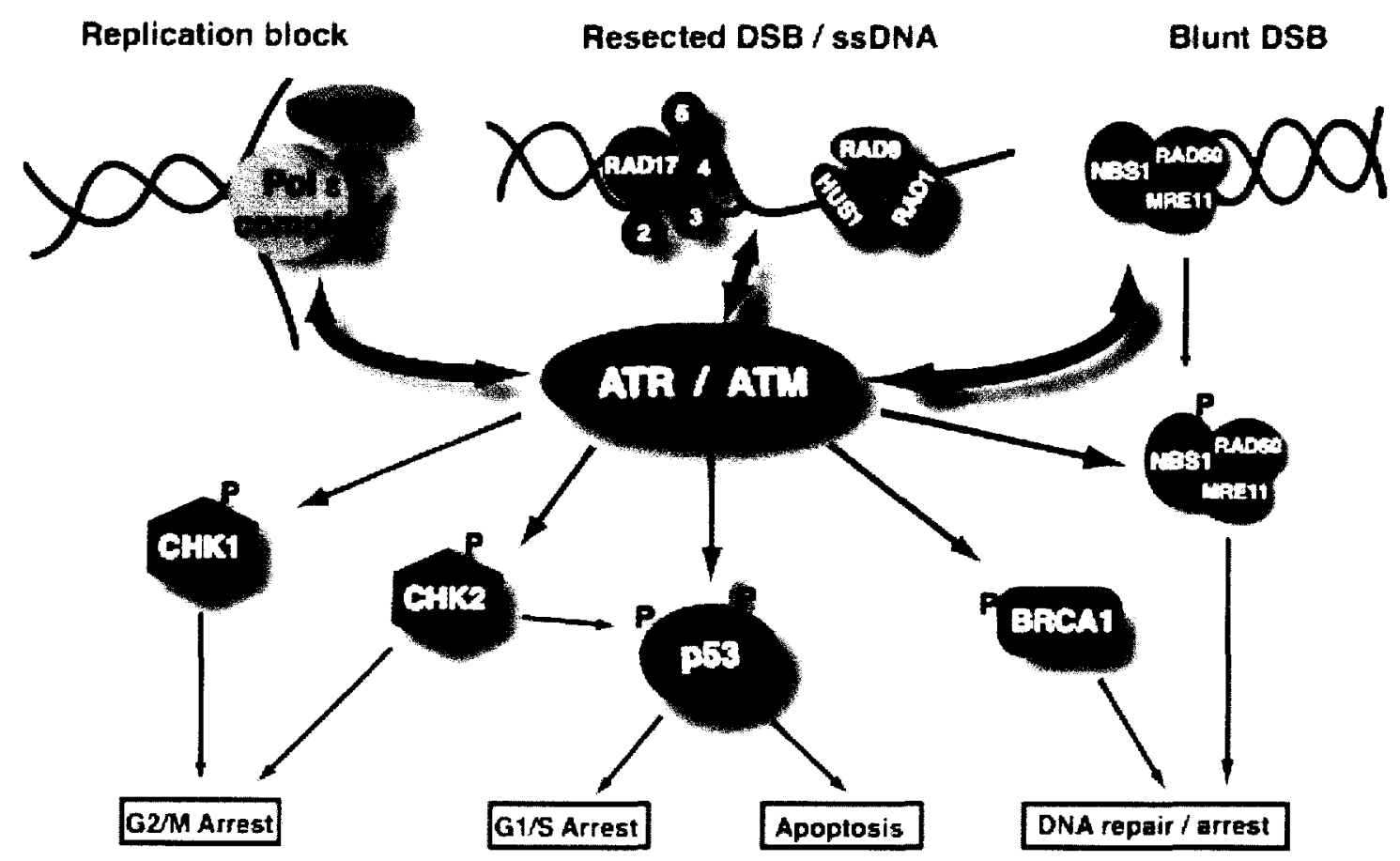

Figure 1-1. ATR and ATM are the central proteins regulating checkpoint responses to various forms of DNA damage. Activation of ATR and its downstream effects are indicated in red, whereas activation and downstream effect of ATM are indicated in blue (not absolute; some exceptions of overlapping function do occur). Signals and downstream effects common to both kinases are designated in purple. Figure from Rhett Michelson. 
ATR and Single-strand DNA Response Complexes.

The ATR-ssDNA checkpoint pathway likely involves four sets of protein complexes that assemble directly or indirectly on DNA to initiate and propagate a signal for arrest and to recruit damage repair proteins. How these four protein complexes interact in concert to mediate a DNA damage response is largely speculative. Like the replicative RFC complex with PCNA, the Rad17-RFC pentamer may bind ssDNA and facilitate the binding of the 9-1-1 heterotrimer to damage; the ATR-ATRIP heterodimer may bind damage independently, as suggested by yeast studies (Rouse \& Jackson, 2000; Kondo et al, 2001; Melo et al., 2001). The Rad17-RFC complex together with the 9-1-1 complex may then serve to recruit substrates, including BRCA1 and its associated proteins, for phosphorylation by ATR. Indeed, in fission and budding yeast, the ATRlike kinase requires components of both the yeast Rad17-RFC complex and the yeast 9-11 complex to phosphorylate Crb2 and Rad9 (Saka et al., 1997; Emili, 1998). Once phosphorylated, BRCA1 may activate downstream protein kinase cascades, aiding in amplification of the damage signal by recruiting additional phosphorylation substrates. It has been reported that ATR-like protein kinases can phosphorylate some substrates, like the $\mathrm{H} 2 \mathrm{AX}$ component of nucleosomes, independent of sensor proteins (Ward \& Chen, 2001). Phosphorylation of other substrates by ATR is dependent on Rad17-RFC, 9-1-1, and BRCA1 complexes, suggesting that these complexes serve to localize some but not all substrates. 
How ATR becomes activated during a checkpoint response is still uncertain, however, Xenopus studies may provide some indication. Guo et al. (2000) purified ATR from egg extracts using DNA-cellulose and found enhanced ATR activity, 10- to 20-fold above that of ATR immunoprecipitated directly from egg extracts. This suggests that binding to DNA-cellulose activates ATR activity or selectively purifies an activated form of ATR (Abraham, 2001).

ATM and Double-strand Break Response Complexes.

The ATM protein kinase, orthologous to Tell in both budding and fission yeast, has received more extensive study than ATR for two reasons: 1) ATM was discovered to be the gene defective in the syndrome AT in 1995, while no association between ATR deficiency and a disease yet exists, and 2) ATM is not essential for cell viability whereas ATR is (Savitsky et al., 1995; Brown \& Baltimore, 2000; de Klein et al., 2000). Cells lacking ATM display chromosomal instability, RDS, and extreme sensitivity to ionizing radiation (IR) and radiomimetic drugs. To account for these phenotypes, AT cells are defective for cell cycle checkpoints at $\mathrm{Gl} / \mathrm{S}, \mathrm{S}$, and G2/M phase transitions in response to radiation-induced damage (reviewed in Abraham, 2001).

In accord with the view that ATM recognizes dsDNA ends, ATM has been shown to associate with non-specific DNA fragments in vitro, demonstrating a modest affinity for linear DNA over supercoiled molecules, and often times localizing at DNA ends (Smith et al., 1999; Costanzo et al;. 2000). At DSBs, ATM may associate with the MRN 
complex of proteins, which could then serve to recruit ATM substrates for phosphorylation, much like the 9-1-1 and Rad17-RFC complexes do for ATR. That ATM and MRN act together at DSBs is inferred from the fact that cells from patients with mutations in $N B S 1$, presenting as Nijmegan breakage syndrome, also exhibit radiosensitivity and chromosomal fragility like AT cells (Bressan et al.,1999), and patients with mutations in MRE11 present with clinical symptoms and cellular defects much like those seen in AT, thus acquiring the name AT-like disorder (Stewart et al., 1999).

Budding yeast studies additionally support the model that ATM and MRN act coordinately at DSBs. Usui et al. (2001) determined that unresected DSBs created during meiosis activate Tell in vivo through the MRX complex, where checkpoint activation is dependent on Mrel1 acting as a damage sensor. The MRX complex consists of Mre11, Rad50, and the budding yeast protein, Xrs2, which is replaced by Nbs1 in mammals.

Understanding the targets of ATM phosphorylation will likely provide great insights into the mechanism of checkpoint activation in response to DSBs (reviewed in Kastan \& Lim, 2000). What can be gleaned at this point, however, is that, at least for two cases, ATM regulates different proteins within the same pathway to ensure the control of a key target. In regulating p53, ATM employs three different means to stabilize the protein. ATM, or a closely associated kinase, has been shown to phosphorylate $\mathrm{p} 53$ on $\mathrm{Ser}^{15}$ in vitro, and most likely also does so in vivo, as AT cells show much reduced levels 
of $\mathrm{p} 53$ phosphorylated at the $\operatorname{Ser}^{15}$ residue in response to IR (Canman et al, 1998). This phosphorylation by ATM likely causes p53 transcriptional activation (Dumaz \& Meek, 1999), while phosphorylation of $\mathrm{p} 53$ at $\mathrm{Ser}^{20}$ by Chk2 acting downstream of ATM, reduces the ability of ubiquitin ligase $\mathrm{Mdm} 2$ to bind p53 thus promoting its stabilization (Haupt et al., 1997; Chehab et al., 2000; Hirao et al., 2000; Shieh et al., 2000). ATM further ensures p53 stabilization by phosphorylating $\mathrm{Mdm} 2$, thereby preventing export of p53 to the cytoplasm where it is degraded (Khosravi et al., 1999). Another 'tri-strategy' approach involves regulation of BRCA1. ATM directly phosphorylates BRCA1 after activation by DSBs caused by ionizing radiation, thought the consequence of this phosphorylation is unknown (Cortez et al., 1999; Gatei et al., 2000). Moreover, activated Chk2, downstream of ATM, additionally phosphorylates BRCA1 on another site that is required for the dissociation of BRCA1 and Chk2 (Lee et al., 2000). ATM also phosphorylates CtIP, forcing dissociation of this inhibitor from BRCAl (Li et al., 2000).

The Molecular Pathways Associate with Replication Forks.

The regulation of DNA replication by checkpoint controls may be of the greatest importance in both genome stability and potentially in cancer therapy. Indeed, a major role of checkpoint proteins may be to stabilize stalled replication forks, since fork collapse can lead to chromosome rearrangements and, thus, genome instability or cell death. Genome instability can activate oncogenes leading to unregulated, abnormal cell growth. Cell death results from loss of genetic material and/or apoptosis. Therefore, both ontogeny and treatment of checkpoint-defective cells may rely to a great degree on 
replication fork biology. Much of the current interest in replication fork biology has been motivated by findings in bacteria where replication fork collapse is thought to be a common feature (occurring about once in every two cell divisions) with RecA acting to restore failed forks by homologous recombination (Cox et al., 2000).

Replication forks slow or stall when they encounter DNA adducts, creating a physical impediment, or when dNTP pools are limiting. Replication disruption elicits four cellular responses: a block to initiation of replication (origin firing), slowing of elongation, maintenance of slowed or stalled replication forks, and/or a block to mitosis (Figure 2). In budding yeast, we now know that checkpoint proteins regulate three of these four responses. After DNA damage, mutants in the key regulators Mec1 and Rad53 fail to block the firing of late origins of replication (Santocanale \& Diffley, 1998), do not stabilize stalled replication forks (Desany et al., 1998; Lopes et al., 2001; Tercero \& Diffley, 2001), and fail to prevent mitotic entry (Weinert et al., 1994). 


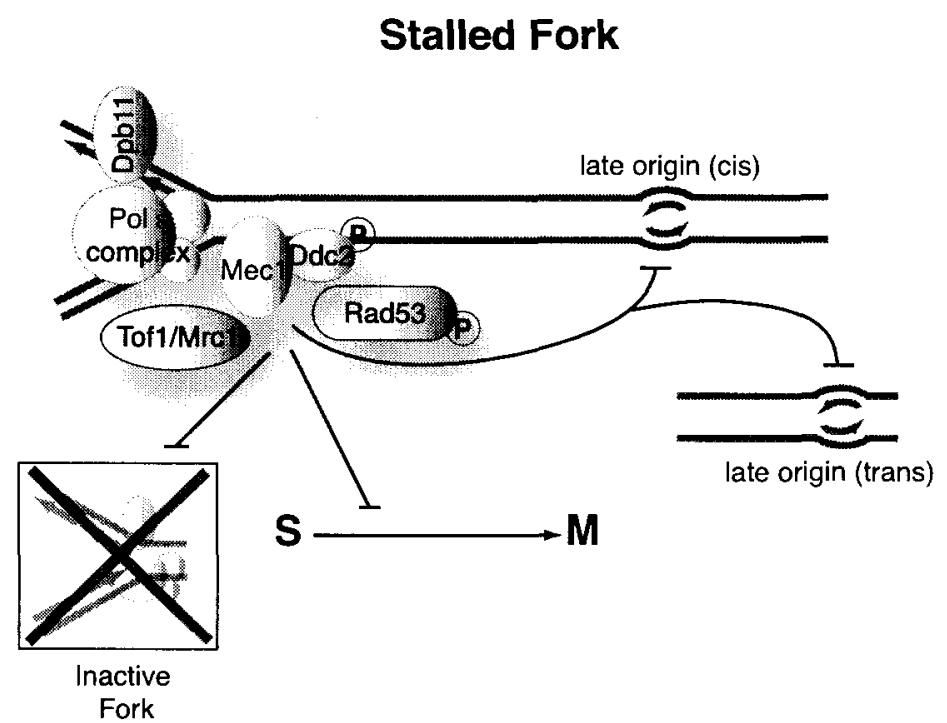

Figure 1-2. A stalled replication fork activates three common checkpoint mechanisms: (a) stabilization of the fork by blocking fork collapse, $(b)$ a block to mitosis, and (c) blockage of late origin firing both in cis and in trans. Figure from Rhett Michelson.

Many of the mechanisms controlling these responses are understood to a limited degree. Surprisingly, slowing of elongation does not require the yeast checkpoint genes; the replication apparatus inherently slows upon encountering damage, likely due to a physical block in fork progression. The S. cerevisiae Mec1 and Rad53 checkpoints are, however, required for the other three responses. Blocking of late origin firing likely occurs though Rad53 phosphorylation of Dbf4-Cdc7, a protein kinase required for the firing of all origins (Weinreich \& Stillman, 1999), and blockage of Cdc25A and Orc function are required in higher cells (Falck et al., 2001). In budding yeast, inhibition of mitosis involves regulation of Esp1 and Pds1, which ultimately prevents cleavage of cohesins between sister chromatids to block chromosomal segregation at mitosis (TinkerKuberg \& Morgan, 1999). In higher eukaryotes, it includes inhibitory phosphorylation of Cdc2 by blocking Cdc25C activity (Sanchez et al., 1997). 
The roles of $\mathrm{Mec} 1$ and Rad53 proteins in preventing catastrophic termination of forks is likely crucial (Desany et al., 1998; Lopes et al., 2001; Tercero \& Diffley, 2001). That checkpoints might regulate stability of replication forks was first suggested by Enoch, Nurse, and Carr (1992) who found two mutants, rad3 and $c d c 2-3 w$, defective in blocking mitosis of S-phase arrested cells in S. pombe. The rad3 mutant dies in S-phase, while the $c d c 2-3 w$ mutant only dies upon entry into mitosis. The $S$. pombe $\operatorname{Rad} 3$ (ATRlike) protein kinase was thereby considered to be required for recovery from replication stress. This recovery function probably entails stabilizing slowed or stalled replication forks, now shown most convincingly in budding yeast studies. The Diffley and Foiani groups independently showed by different methods using $S$. cerevisiae that wild-type cells slow the rate of replication when treated with MMS to induce damage or with hydroxyurea (HU) to deplete dNTP pools, but fork stability is maintained such that replication by the stalled or slowed fork can resume when cellular conditions permit (Lopes et al., 2001; Tercero \& Diffley, 2001). In Mec1-deficient cells, however, replication forks collapse and fail to resume replication. One can now infer that stability of forks is a primary determinant for damage-sensitivity in checkpoint mutants from the following observations. Hypomorphic mecl mutants and mrcl null mutants are relatively resistant to MMS, yet fail to block late origin firing or entry into mitosis (Alcasabas et al., 2001; Paciotti et al., 2001). From these findings, it is surmised that mecl and $\mathrm{mrcl}$ mutants are damage-sensitive due to their inability to stabilize replication forks and not owing to defects in blocking replication from late origins or preventing mitotic entry. An 
additional implication is that checkpoint proteins may act efficiently in cis to retain a fork, but require more activity to act in trans to block late origin firing and mitotic progression. For the latter purpose, Mrcl appears to be a potentiator or amplifier of the damage signal in S-phase, much as Rad9 is in response to DNA damage in G2.

Interestingly, one can make a similar inference for mammalian cells in that fork stability, and not cell cycle progression per se, may be a key determinant of damage sensitivity. Mutants in ATM are defective for inhibition of origin firing or RDS. Suppressors of $\mathrm{ATM}^{/ 2}$ mutant strains have been identified that restore inhibition of late replication origins, yet remain damage sensitive (Kamijo et al., 1999).

The mechanism of fork stabilization is unknown, although it may involve regulation of DNA primase (Marini et al., 1997), as is the exact fate of stalled, intact replication forks. Recently, an interaction was reported between a Holliday resolving protein and the Cdsl checkpoint protein kinase in fission yeast (Boddy et al., 2000). It is plausible that checkpoint proteins stabilize forks by preventing cleavage of some intermediates. Thus, in a larger scheme, checkpoint proteins may prevent repair of stalled forks by recombination pathways.

\section{The Gl Checkpoint.}

Most eukaryotic cells damaged in G1 generate a pronounced delay prior to Sphase. This arrest in G1 allows vital time for repair and prevents replication of a 
damaged template. Otherwise, uninhibited DNA replication would convert one gapped chromosome into 2 sister chromosomes, one of which contains a DSB. While the role of the G1 checkpoint in cell physiology seems clear, it is often the subject of debate because the role of this checkpoint is much different in higher eukaryotes than in lower ones.

In budding yeast, the G1 checkpoint exists but is very weak; damage induces a delay that lasts far less than an hour and most damage remains unrepaired (Siede et al., 1994; Fitz Gerald et al, 2002). For example, a double-strand break induces no detectable delay in G1 but leads to a prolonged arrest in G2 (Weinert et al., 1994). This may reflect the ability of budding yeast to efficiently repair DSBs by homologous recombination using the intact sister homolog. What little delay is detected in G1 involves Rad53dependent phosphorylation of the Swi4/6 transcription factors. This phosphorylation inhibits transcription of G1 cyclins, thereby slowing entry into S-phase (Sidorova \& Breeden, 1997).

In contrast to the weak G1 arrest in yeast, DNA damage induces a very robust G1 arrest in higher eukaryotes from Xenopus to mammals, recognized for its interaction with the tumor suppressor p53. This G1 arrest now appears to have two waves of action (Figure 3). The first immediate delay provides time for repair but lasts only several hours, whereas a slower and sometimes irreversible delay may function to remove cells from the cell cycle (Mailand et al., 2000; Bartek \& Lukas, 2001). 


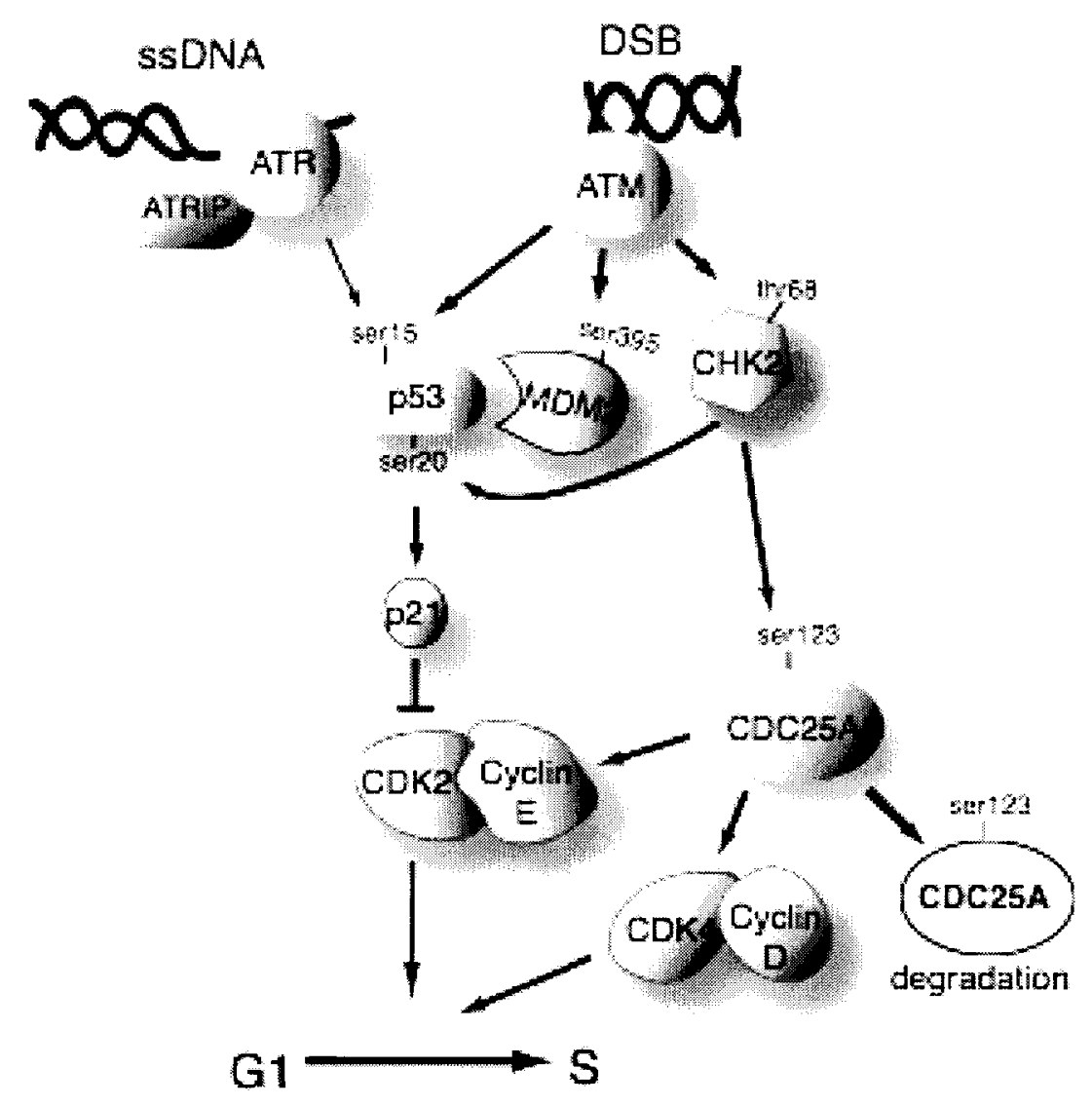

Figure 1-3. The G1 checkpoint in mammalian cells primarily functions to block Cdk2cyclin $\mathrm{E}$ activity. This is achieved by stabilizing $\mathrm{p} 53$ and degrading $\mathrm{Cdc} 25 \mathrm{~A}$ to maintain Cdk2 inhibitory phosphorylation. Gray arrows denote functions that are lost upon activation of the checkpoint cascade, and labeled amino acids on proteins indicate sites of phosphorylation. Figure from Rhett Michelson.

The first delay occurs within minutes after damage, employs post-translational modification of proteins, and is p53-independent. ATM/ATR-dependent activation of Chk1/Chk2 leads to phosphorylation of Cdc25A, thereby priming it for ubiquitination and proteasome destruction (Mailand et al., 2000). Loss of Cdc25A consequently regulates $\mathrm{Cdk} 2$ by maintaining its inhibitory phosphorylation on $\mathrm{Tyr}^{15}$ and inhibiting its 
association with cyclin E, a necessary step for progression into S-phase. An elegant set of experiments using a cell-free system derived from Xenopus eggs showed that Cdk2cyclin $\mathrm{E}$ inhibition also prevents complete formation of pre-replicative complexes on DNA to also inhibit the start of replication (Costanzo et al., 2000). All pre-replication complex components are present - ORC, Cdc6, CDC7, and MCM proteins - with the exception of $\mathrm{Cdc} 45$, which requires $\mathrm{Cdk} 2$-cyclin $\mathrm{E}$ activity for assembly at origins where it then functions to attract DNA polymerases. Cdc25A also regulates Cdk4 activity and, as indicated by a much earlier study performed with UV treatment, destruction of $\mathrm{Cdc} 25 \mathrm{~A}$ results in maintenance of the $\mathrm{Tyr}^{17}$ inhibitory phosphorylation on $\mathrm{Cdk} 4$ that also prevents S-phase entry (Terada et al., 1995).

A second G1 delay mechanism again involves ATM/ATR and CHK1/CHK2, but takes several hours to initiate because regulation for this block to S-phase entry occurs at the transcriptional level. Here, ATM/ATR and CHK1/CHK2 function to activate and stabilize $\mathrm{p} 53$, as discussed earlier, causing transcriptional induction of $\mathrm{p} 21$, which then inhibits the Cdk2-cyclin E complex (Sherr \& Roberts, 1999; Ekholm \& Reed, 2000). The p53-dependent block in G1 operates to maintain the arrest initiated by the $\mathrm{Cdc} 25 \mathrm{~A}$ pathway to allow sufficient time for repair of DNA damage. Questions remain as to how recovery from arrest is achieved or whether, in fact, the p53-dependent delay is reversible in all cell types (Di Leonardo et al., 1994; Carr, 2000). Another major lingering question is whether or not these two checkpoint cascades can occur independently; that is, can limited damage that requires little time for repair induce only the post-translational 
cascade for a brief delay, or are both cascades always activated together, regardless of the extent of damage (Bartek \& Lukas, 2001)?

The issue of whether G1 arrest or apoptosis is more important in mammalian cells in preventing cancer is yet unresolved. If both mechanisms function to remove damaged cells from cycling, then both may play prominent roles in cancer evasion.

The S-phase Checkpoint.

Description of S-phase checkpoint pathways is now taking shape, driven predominantly by studies of budding and fission yeast. Based on details inferred from genetic observations, we present models of each organism in an attempt at deriving unifying mechanisms. We note that details of the fates of forks in the two yeasts are similar, with some significant differences, and the possibility that mammalian cells exhibit similar features will be addressed herein.

A traditional view held by many is that cells in S-phase activate one of two checkpoint pathways to signal aberrant replication forks and/or DNA damage: the intra-S checkpoint, mediated by the DNA damage-responsive genes, and the replication checkpoint, mediated by polymerase-associated genes. Due to key features shared by these two pathways, we believe that these two responses can be integrated into one pathway, termed simply the S-phase checkpoint. Much of this model is based on key 


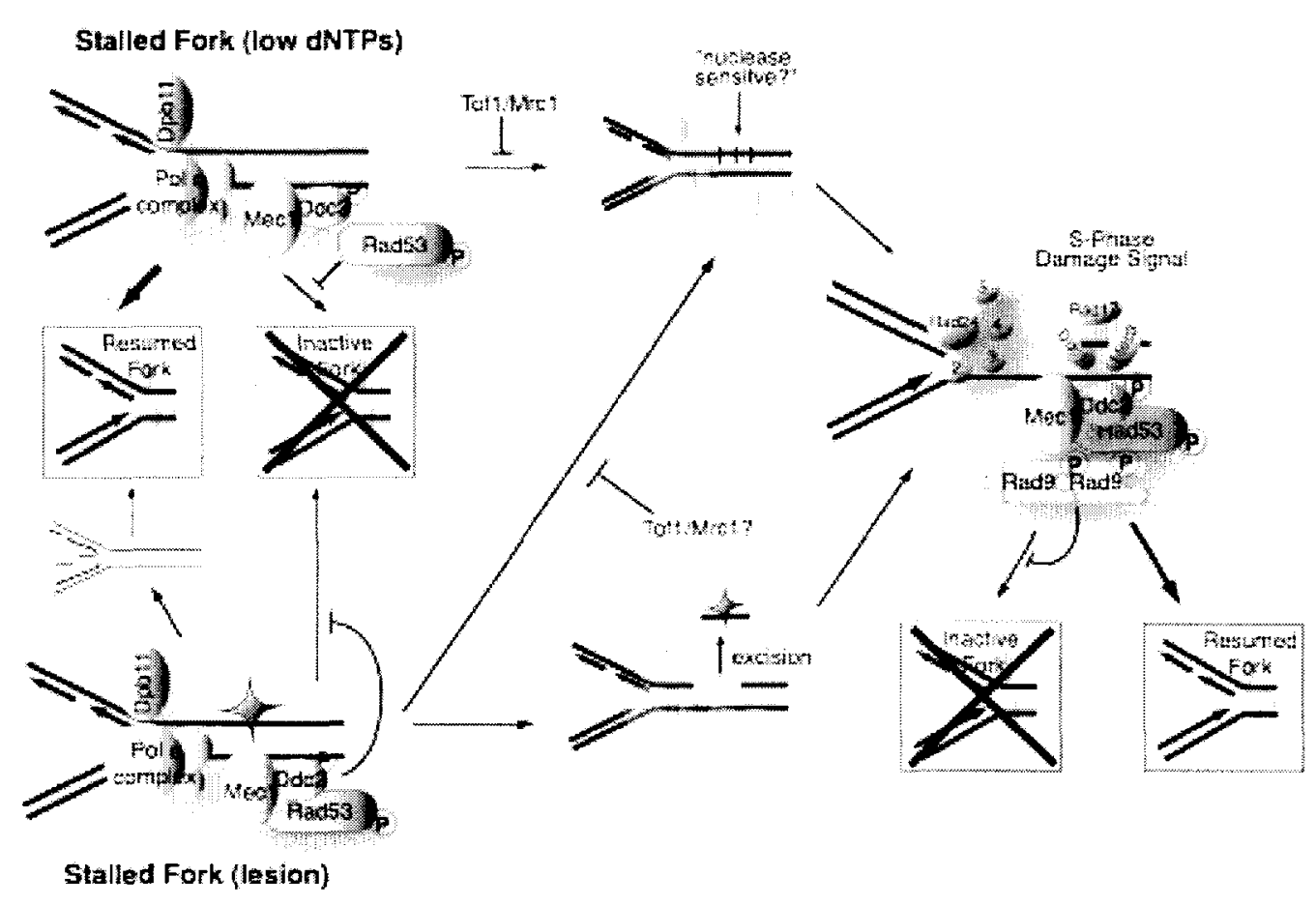

Figure 1-4. In budding yeast, replication forks stall upon encountering DNA lesions or low dNTP pools, signaled by proteins localized at sites of replication. Tof 1 and Mrcl block the conversion of stalled forks prone to form alternative structures. Potential attack by nucleases or lesion processing induces activation of the checkpoint sensors that specifically recognize DNA damage to further maintain fork stability. Figure from Rhett Michelson.

observations of alternative pathways in S-phase control both in budding yeast (Figure 4) and fission yeast (Figure 5; Alcabasas et al., 2001; Foss, 2001; Tanaka \& Russell, 2001).

The first step in S-phase checkpoint activation is stalling of replication forks due to either depletion of dNTPs or to encountering DNA damage. We consider these two forms of damage similar because they both slow replication fork progression and either insult can culminate in fork collapse in checkpoint mutants. In support, like replication in 
the presence of $\mathrm{HU}$ that depletes dNTP pools, exogenous DNA damage (MMS treatment)

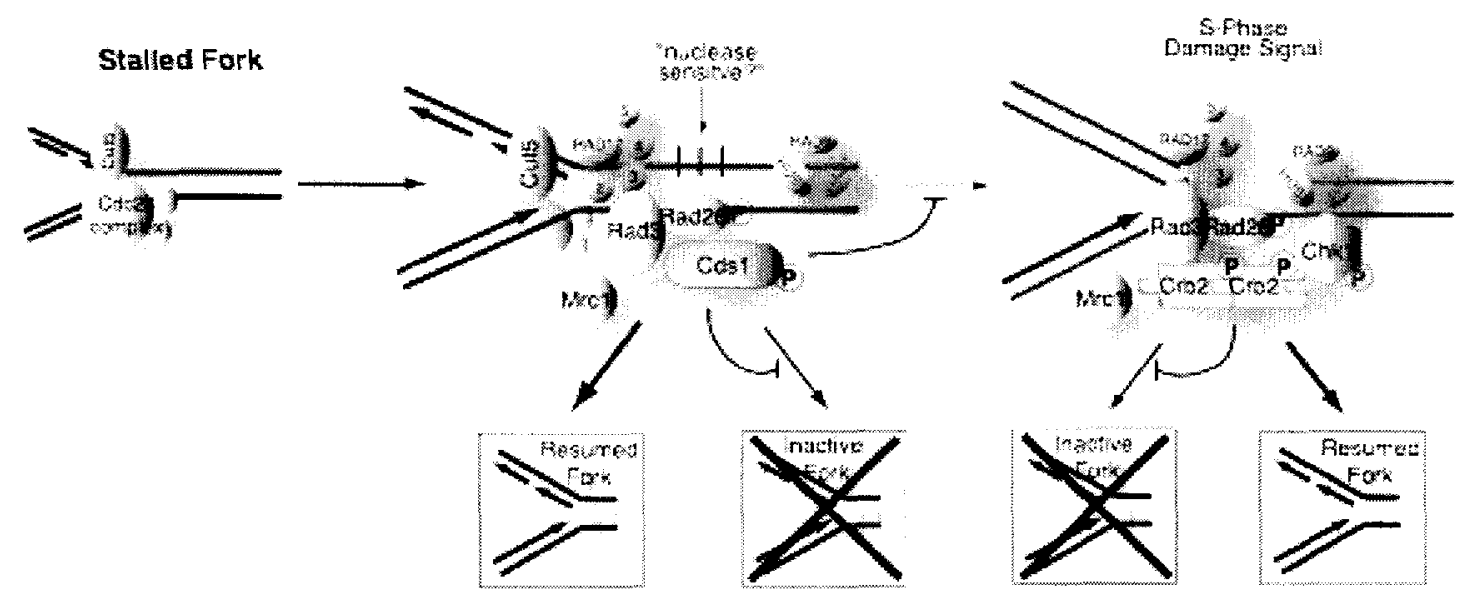

Figure 1-5. In fission yeast, stalled replication forks are immediately converted to alternative structures that are blocked from possible nuclease attack and collapse. Mrc1 additionally functions to block possible nuclease attack. Those forks that undergo nuclease processing activate the checkpoint sensors that respond to DNA damage to maintain fork stability. Figure from Rhett Michelson.

also slows elongation of already assembled replication forks, as determined by analysis of conversion of heavy-heavy-labeled DNA to heavy-light-labeled DNA over time at specific sequences downstream of origins (Tercero \& Diffley, 2001). In budding yeast, slowing or stalling of DNA replication forks by low dNTPs or damage signals actives the S-phase checkpoint pathway by proteins that are localized at the fork sites. The proteins associated with replication forks that display checkpoint activity include the DNA helicases Sgs1 and Top3 (Frei \& Gasser, 2000; Chakraverty et al., 2001); Pol2, a subunit of DNA pol $\varepsilon$ that is essential for DNA replication and implicated in DNA repair (Navas et al., 1996); Dpb11, a protein that interacts with and recruits Pol2 to replication origins (Wang \& Elledge, 2002); Drc1, an essential protein for DNA replication that interacts 
with Dpb1 1 (Wang \& Elledge, 1999); and RFC subunits 2, 3, 4, and 5 (Noskov et al., 1998; Shimada et al., 1999; Naiki et al., 2000; Kim \& Brill, 2001). These proteins, along with contributing functions from Mrc1 and Tof1, somehow activate Mec1 and $\operatorname{Rad} 53$ to carry out the common S-phase responses detailed in Figure 4 (Alcasabas et al., 2001; Foss, 2001).

Alternatively, we envision stalled forks as being subject to another fate: conversion to a form that activates other checkpoint proteins, like $\operatorname{Rad} 9$, to somehow maintain or restore fork stability. Genetic analysis suggests that the primary stalled fork is prevented from conversion to this other form by Tofl and Mrc1, because in the absence of Tof1 or Mrc1, S-phase checkpoints become dependent on Rad9 (Alcasabas et al., 2001; Foss, 2001). These forks can resume replication provided that they activate Mec1 and Rad53 for fork stabilization.

We suggest that a stalled fork at a lesion is subject to an additional fate. Lesion processing followed by replication generates a fork with a DSB. In this situation, the DNA damage created is sufficient to signal the damage checkpoint proteins, such as Rad9, that then prevent late origin firing and spindle elongation via Mec1 and Rad53. Therefore, the damage-recognition checkpoints serve as a backup to the replication protein checkpoints. This model accounts for why damage induces a weak Rad9dependent S-phase response that becomes greatly enhanced in cells lacking either Tof 1 or Mrc1 (Alcasabas et al., 2001; Foss, 2001). 
We propose that in fission yeast, and therefore perhaps in mammalian cells, a similar, though not identical, progression of replication fork conversions may occur (Figure 5). First, replication forks blocked in fission yeast always activate Rad3 using the damage sensor proteins and Mrcl (Tanaka \& Russell, 2001). We therefore infer that a stalled replication fork may convert constitutively to some altered form that then signals using damage sensor proteins. Next, this fork intermediate may convert to another form - a process actively prevented by Cds1/Chk2 (Lindsay et al., 1998). This is supported by the key observation that Cdsl mutants are not appreciably sensitive to hydroxyurea, and when HU-treated require Chk1 activity for viability (Lindsay et al., 1998; Martinho et al., 1998). We suggest, then, that Cds 1 prevents the conversion of the first broken fork structure, while Chk1 acts as a failsafe mechanism, like budding yeast $\operatorname{Rad} 9$, to rescue any forks that have converted to the second altered fork form. Tests of these models linking DNA fork structures to protein functions are close at hand. Foiani and colleagues (submitted) have shown that replication forks formed in yeast can be isolated and their structures determined by electron microscopy.

In mammalian cells, some genetic basis of S-phase checkpoints has been well documented (Painter \& Young, 1980), though the underlying mechanisms remain largely obscure. Brcal, ATM, and MRN complexes are involved after $\gamma$-irradiation, and ATR is likely involved after UV damage (Lim et al., 2000; Zhao et al., 2000; Falck et al., 2001; Ward \& Chen, 2001; Xu et al., 2001; You et al., 2002). Cdc25A is also implicated as a downstream effector since it appears to regulate ORC complexes at initiation (Falck et 
al., 2001). It is unknown whether mammalian replication forks experience similar options of conversion as in yeast (Figure 4). Differences between lower and higher eukaryotes include much longer tracts of DNA between origins, suggesting that processivity is more critical in mammals and may require other or additional forms of regulation by checkpoints. Whatever mechanisms operate in eukaryotic cells, fork stability mediated by checkpoint proteins is likely to be critical for genome stability. Furthermore, if replication defects are ongoing and persistent in cancer cells, then the potential for developing therapeutic strategies based on defects in replication fork regulation takes precedence.

\section{The G2/M Checkpoint.}

Though well characterized in both yeast systems, the details of G2/M arrest in higher eukaryotic cells are still being sorted out. The picture that has emerged thus far is that fission yeast and higher eukaryotes, including mammalian cells and Xenopus, both target $\mathrm{Cdc} 2$ to maintain its inhibitory phosphorylations as the means by which to block $\mathrm{G} 2 / \mathrm{M}$ transition. This is achieved by targeting various parallel pathways - including the phosphatases that promote mitosis, the kinases that block Cdc2 function, and other proteins that regulate these regulators - which all converge to regulate $\mathrm{Cdc} 2$ activity (Figure 6). 


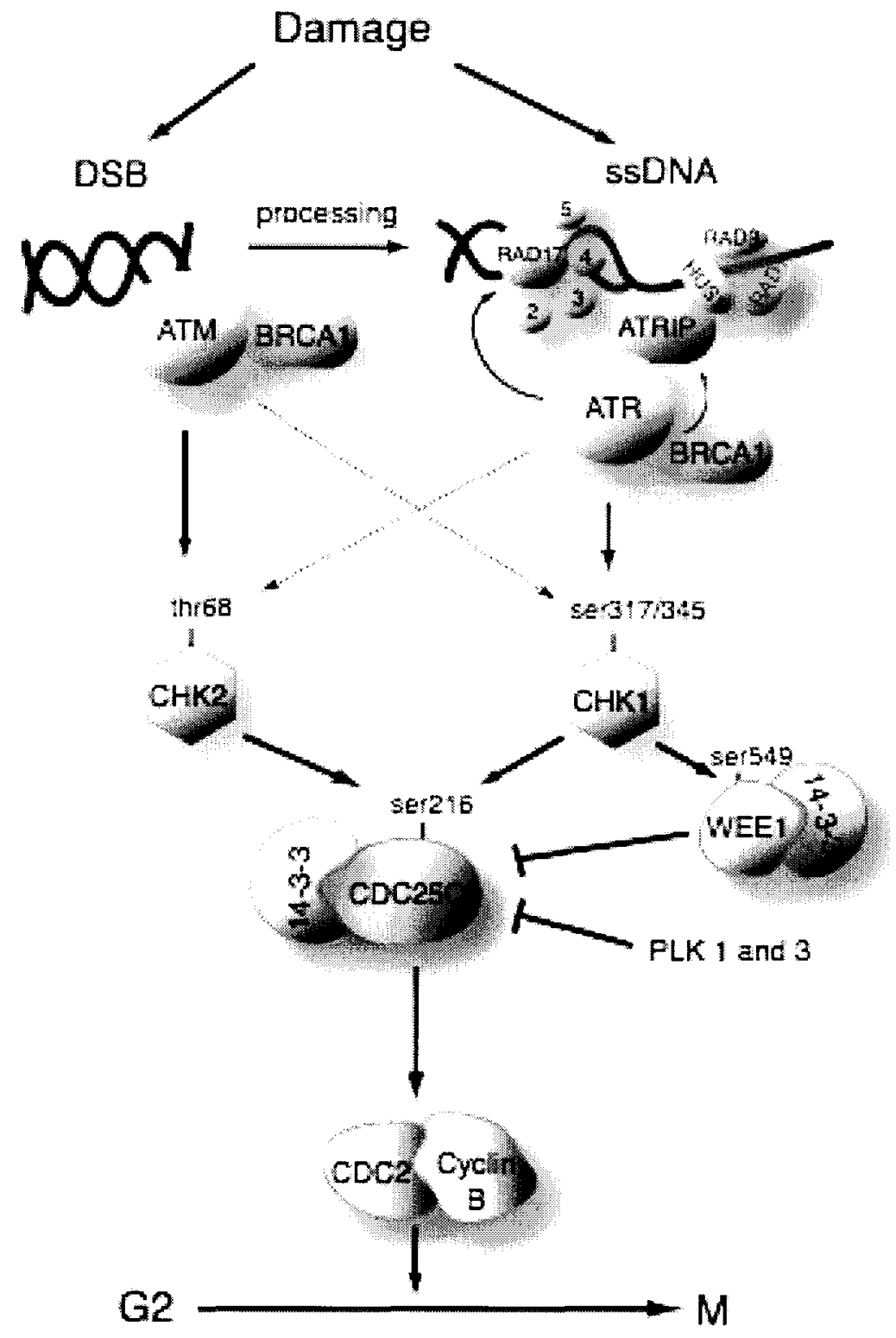

Figure 1-6. The G2 checkpoint in mammalian cells primarily functions to block Cdc2cyclin $\mathrm{B}$ activity. The common means of maintaining $\mathrm{Cdc} 2$ inhibitory phosphorylation is by blocking $\mathrm{Cdc} 25 \mathrm{C}$ phosphatase activity, namely by promoting its association with 143-3 proteins. Gray arrows denote functions that are lost upon activation of the checkpoint cascade, and labeled amino acids on proteins indicate sites of phosphorylation. Figure from Rhett Michelson. 
DNA damage in mammalian cells causes the activation of four different downstream kinases that independently act to block the activity of $\mathrm{Cdc} 25 \mathrm{C}$, the phosphatase that promotes mitosis by removing phosphates at $\mathrm{Cdc} 2$ inhibitory sites. First, activation of ATR by damage leads to Chk1 phosphorylation (Guo et al., 2000). Chk1 then negatively regulates Cdc25C by phosphorylating it at $\operatorname{Ser}^{216}$ (Peng et al., 1997; Sanchez et al., 1997). This phosphorylation of Cdc25C creates a binding site for 14-3-3 proteins, and in the bound state renders Cdc $25 \mathrm{C}$ catalytically inactive, sequestered in the cytoplasm, or both (Graves et al., 2001; Lopez-Girona, 2001). In addition to activation by ATR, a recent paper suggests that Chk1 can be activated by BRCA1 to induce a G2/M arrest, though this has yet to be confirmed by in vitro analysis (Yarden et al., 2002). By a second mechanism, ATM activation by DNA damage in G2 leads to Chk2 activation that also phosphorylates $\mathrm{Cdc} 25 \mathrm{C}$ at $\operatorname{Ser}^{216}$ to block its function (Matsuoka et al., 1998; Zhou et al., 2000). And now two new regulators of Cdc25C are emerging - both members of the Polo-like kinase (Plk) family. Plk3 resembles Chk1 and Chk2, as it is activated in an ATM-dependent manner, physically interacts with Cdc25C, and phosphorylates this protein at $\mathrm{Ser}^{216}$ to inhibit its activity (Smits et al., 2000; Xie et al., 2001). Unlike the kinases that inhibit Cdc25C activity by $\mathrm{Ser}^{216}$ phosphorylation, $\mathrm{Plk} 1$ is recognized for its role in promoting mitotic entry by phosphorylating $\mathrm{Cdc} 25 \mathrm{C}$ to activate it. It is now believed that ATR and ATM both function to phosphorylate Plk1, perhaps via Chkl and Chk2 activation, to block Plk1 activation of Cdc25C (Smits et al., 2000; van Vugt et al., 2001). Plk1 inhibition correlates with a decrease in Cdc2-cyclin B kinase activity, and this inhibition is completely blocked with caffeine, a known inhibitor of ATM/ATR. 
Additionally, expression of unphosphorylatable Plk1 can not be inhibited in the presence of DNA damage and does not initiate a G2/M arrest, further implicating Plk1 as another regulator of Cdc25C activity (Smits et al., 2000). Adding another level of G2/M control, Plk1 protein stability now appears to be regulated by the checkpoint protein Chfr, which has been shown to delay entry into mitosis when cells are under mitotic stress (Scolnick \& Halazonetis, 2000; Kang et al., 2002). Using Xenopus cell-free extracts, Kang et al. showed that Chfr ubiquitinates the human Plk1 to target it for degradation thereby inhibiting Cdc25C activation, resulting in a delay in $\mathrm{Cdc} 2$ activation. The mechanism of Chfr activation has yet to be determined.

While the majority of 14-3-3-bound Cdc25C localizes to the cytoplasm, some Cdc25C does remain in the nucleus (Zhou et al., 2000), implying that other mechanisms are required for inhibition of $\mathrm{Cdc} 25 \mathrm{C}$ phosphatase activity. In mammalian cells, inhibition may be accomplished by the Cdk inhibitor p21 (Ando et al., 2001). p21 is known to bind PCNA to inhibit CDK catalytic activity (Xiong et al., 1992; Harper et al., 1993). Recently, Cdc25C was shown to also interact with PCNA in vivo (confirmed by in vitro analysis) at the $\mathrm{G} 2 / \mathrm{M}$ transition, but the purpose for this interaction is yet unknown (Kawabe et al., 2002). It is possible that PCNA acts as a platform to mediate protein interactions and that binding of $\mathrm{p} 21$ and $\mathrm{Cdc} 25 \mathrm{C}$ to a PCNA-Cdc2-cyclin B complex is a mutually exclusive event (Ando et al., 2001). Therefore, p21 interaction with PCNA-Cdc2-cyclin B may exclude Cdc25C from interacting with $\mathrm{Cdc} 2$ to dephosphorylate it for mitotic progression. By an additional method, p21 may act 
directly on Cdc2. Although p21 does not affect the inhibitory phosphorylation sites $\left(\mathrm{Thr}^{14}\right.$ and $\mathrm{Tyr}^{15}$ ) on $\mathrm{Cdc} 2, \mathrm{p} 21$ does block the activating phosphorylation of $\mathrm{Cdc} 2$ on Thr ${ }^{161}$, mediated by the Cdk-activating kinase, CAK (Smits et al., 2000).

Though 14-3-3 proteins regulate $\mathrm{Cdc} 25 \mathrm{C}$ by blocking its $\mathrm{Cdc} 2$ phosphatase activity upon binding, 14-3-3 proteins may also bind to other proteins to regulate Cdc2 activation to maintain a $\mathrm{G} 2 / \mathrm{M}$ arrest. In addition to $\mathrm{Cdc} 25 \mathrm{C}, 14-3-3$ proteins are now known to bind Weel (Lee et al., 2001). Xenopus extract experiments allowed the initial in vitro observation that $14-3-3$ proteins bind to Wee 1 when it is phosphorylated at $\operatorname{Ser}^{549}$ and that this site is phosphorylated by Chkl. By both recombinant protein and immunodepletion analysis, Chk1 phosphorylation of Weel serves to promote 14-3-3 binding that significantly enhances the inhibitory kinase activity of Weel toward Cdc2. By another mechanism identified in colorectal cell lines, p53 mediates 14-3-3o induction after DNA damage independent of $\mathrm{p} 21 ; \sigma$ expression is needed for $\mathrm{G} 2 / \mathrm{M}$ arrest and to prevent 'mitotic catastrophe' (Hermeking et al., 1997; Chan et al., 1999). Based on Western analysis of immunoprecipitated complexes, 14-3-3 $\sigma$ appears to bind to the Cdc2-cyclin B complex in order to sequester it in the cytoplasm to maintain G2 arrest, though arrest can be initiated in the absence of 14-3-3o or p21 (Chan et al., 1999). Whether the same mechanism functions in non-colorectal cells is unknown.

Just as Wee1 is subject to checkpoint-dependent regulation to stimulate its inhibitory effects on Cdc2, Mytl may also surface as a target of checkpoint mechanisms 
in mammalian cells. Mytl is down-regulated in a cell cycle-dependent manner through phosphorylation by Akt, leading to the meiotic G2/M transition (Okumura et al., 2002). Overexpression of Myt1 induces a G2 delay by retaining Cdc2-cyclin B in the cytoplasm (Liu et al., 1999). Regulation of Myt1 by the checkpoint components, however, has yet to be demonstrated in mammalian cells, though this regulation has been established for the fission yeast ortholog Mik1. After DNA damage or replication defects, Mik1 protein accumulates in a Chk1-dependent manner that is required for both initiation and maintenance of a G2/M arrest (Baber-Furnari et al., 2000; Rhind \& Russell, 2001).

Regulation of cyclin B may also contribute to the G2/M arrest (Bulavin et al., 2002). Studies from two laboratories demonstrated that induction of DNA damage leads to retention of cyclin B in the cytoplasm and that forcing nuclear localization of cyclin B partially abrogated the G2/M damage-induced arrest (Jin et al., 1998; Toyoshima et al., 1998). Moreover, cyclin B and Cdc2 are both transcriptionally down-regulated upon checkpoint induction, correlating with a reduction in protein, helping to reinforce the G2 arrest (Taylor et al., 1999; Crawford \& Worms, 2001).

In response to UV damage, yet another distinct pathway is activated to induce a G2/M delay: p38 is rapidly activated by UV radiation. p38 subsequently phosphorylates $\mathrm{Cdc} 25 \mathrm{~B}$ at $\mathrm{Ser}^{309}$, demonstrated both in vitro and in vivo, to induce 14-3-3 binding, which then leads to the arrest response (Bulavin et al., 2001). 14-3-3 binding blocks access to the catalytic site of the enzyme to directly inhibit Cdc25B activity (Forrest \& 
Gabrielli, 2001). Notably, p38 apparently plays no role in affecting 14-3-3 binding to Cdc25C in vivo (Bulavin et al., 2001).

The budding yeast G2/M checkpoint arrests cells principally at the metaphaseanaphase transition, and the effectors targeted to cause arrest differ from those in fission yeast and higher eukaryotes. This checkpoint primarily halts arrest by targeting Pds 1 for phosphorylation, consequently stabilizing the protein to prevent degradation of chromatin cohesions (Yang et al., 1997; Yinker-Kulberg \& Morgan, 1999; reviewed in Amon, 2001). If future work demonstrates that the anaphase promoting complex (APC) in higher eukaryotes is subject to checkpoint regulation in response to DNA damage, the polo-like kinases being likely targets (Sanchez et al, 1999; Smits et al., 2000), then insights from budding yeasts mechanisms can be drawn to direct experimentation in mammalian cells or Xenopus egg extracts.

\section{DNA Damage Repair and Recovery.}

DNA Checkpoints and Their Link to Repair.

The checkpoint proteins arrest the cell cycle in the presence of DNA damage, presumably to allow the cell time for repair. Given this, it is attractive to speculate that the checkpoints do not simply act as a switch for arrest, but also can directly recruit DNA repair machinery or perhaps even play direct roles in repairing DNA. A role in repair is borne out by the fact that checkpoint deficient cells temporarily arrested with drugs after infliction of DNA damage are not as viable as similarly treated wild-type cells (reviewed 
in Rotman \& Shiloh, 1999). Thus, checkpoint proteins clearly have roles above and beyond that of inducing cell cycle arrest and fork stability, presumably in DNA repair. Here we present the few direct links between checkpoint machinery and DNA repair mechanisms uncovered thus far.

At an indirect level, yeast and mammalian cells contribute to repair responses by inducing transcription of a number of genes encoding proteins involved in repair (Foiani et al., 2000; Li et al., 2000; Bartek \& Lukas, 2001; Gasch et al., 2001; Schramke et al., 2001; Ren et al., 2002). Checkpoints also regulate dNTP pools, perhaps a common feature involved in DNA repair, since blocking dNTP regulation results in enhanced damage sensitivity (Weinert, 1998; Tanaka et al., 2000; Zhao \& Rothstein, 2002).

Hard evidence supporting the notion that checkpoint and repair proteins interact to enhance cell survival is scarce, while soft evidence abounds. Based on inference, a number of the checkpoint components are implicated in repair, and we will provide a few prominent examples. ATM is linked to the repair of DNA damage by homologous recombination (HR) based on the fact that AT cells defective in HR components (rad54 mutation) are no more sick than AT cells, whereas AT cells defective for nonhomologous end-joining (NHEJ; $k u 70$ mutation) show drastic increases in chromosomal instability (Morrison et al., 2000). The inference is that $\mathrm{ATM}^{-/}$cells are already defective for HR, resulting in no increased defect upon rad54 mutation, while they are proficient for NHEJ. BRCA1, also implicated in HR repair (Moynahan et al, 1999) as 
well as transcription-coupled repair (Gowen et al., 1998; Abbott et al., 1999), is thought to be involved in various repair processes. BRCA1 transiently associates with a number of repair proteins - including mismatch repair proteins, Nbs1, and the BLM helicase - in what has been termed the $\underline{B} R C A 1$-asssociated genome surveillance complex (BASC); a number of these proteins co-localize with BRCA1 after DNA damage treatment (Wang et al., 2000). Colocalization after damage induction has also been demonstrated for BRCA1 and Rad51 (Bhattacharyya et al., 2000), Brcal and PCNA (Scully et al., 1997), and BRCA1 and the MRN complex (Zhong et al., 1999). Based on the DNA damagesensitivity of Mre11-Rad50-Nbs1 mutants, their ability to process DSBs, and their genetic grouping with the Rad52 epistasis group, it was assumed that the MRN complex also plays a role in HR. Curiously, however, numerous studies do not attest to the MRN complex as contributing significantly to repair either by HR or by NHEJ (reviewed in D’Amours \& Jackson, 2002).

One protein that we think likely to be involved in both signaling and repairing damage is RPA, the heterotrimeric ssDNA binding protein. RPA contributes essential functions to DNA replication, repair, and recombination (reviewed in Iftode et al., 1999). Studies in both mammals and yeast reveal that the middle RPA subunit, Rpa2, is phosphorylated by ATM (DNA-PK phosphorylation has also been demonstrated) following fork stalling and DNA damage induction, and this phosphorylation abrogates DNA replication as measured by in vitro SV40 replication (Brush et al., 1996; Gately et al., 1998; Oakley et al., 2001; Wang et al., 2001). Since Rpa2 phosphorylation is likely 
directly ATM-dependent and, at least in budding yeast, downstream factors ( $\operatorname{Rad} 9$ and Rad53) are not required for this event, Rpa2 phosphorylation may be necessary to stabilize stalled replication forks or to halt replication until DNA damage is repaired. The involvement of Rpa2 phosphorylation in mediating DNA repair is, as yet, unclear. Interestingly, in budding yeast, it has been shown that the large RPA subunit, Rpal, is additionally phosphorylated by $\mathrm{Mec} 1$, and this event does require $\operatorname{Rad} 9$ and the downstream kinase Rad53, placing the event farther down the checkpoint cascade (Brush \& Kelly, 2000). Also, whereas Rpa2 is normally phosphorylated during the cell cycle, as well as in response to damage, Rpal is only phosphorylated upon genotoxic and HU insult and the extent of phosphorylation correlates with the amount of damage induced. Given the late timing and modulation by levels of DNA damage within the cell, Rpal phosphorylation mediated by Mecl may be necessary for the initiation of DNA repair (Longhese et al., 1996).

The only conclusive evidence tying the checkpoint response directly to DNA repair is that of $\operatorname{Rad} 55$ activation in budding yeast and $\mathrm{Crb} 2$ regulation in fission yeast. Rad55, a recombinational repair protein, undergoes specific phosphorylation mediated by Mecl following treatment by numerous damaging agents (Bashkirov et al., 2000). Mutant rad55 cells are proficient for arrest and induced gene expression in response to DNA damage, but a mec1 mutant that cannot phosphorylate Rad55 shows an 88 -fold reduction in the amount of induced recombination to repair damage. That Rad55 is a terminal substrate of the checkpoint cascade and that major repair defects are evident 
when it is not phosphorylated, directly links checkpoint and repair processes. In fission yeast, the checkpoint protein $\mathrm{Crb} 2$ is phosphorylated at $\mathrm{Thr}^{215}$ by $\mathrm{Cdc} 2$-cyclin $\mathrm{B}$ during mid-mitosis (Esashi \& Yanagida, 1999). This phosphorylation event is necessary for damage-inducible hyperphosphorylation of $\mathrm{Crb} 2$, a step leading to Cdc2-cyclin $\mathrm{B}$ stabilization to induce $\mathrm{G} 2 / \mathrm{M}$ arrest. A recent report shows that $\mathrm{Cdc} 2$-cyclin B promotes homologous recombination by two means (Caspari et al., 2002). At an early step, defects in the $\mathrm{Cdc} 2$-cyclin $\mathrm{B}$ complex abrogate the formation of recombination protein foci; at a later stage, these defects in Cdc2-cyclin B cause aberrant hyper-recombination mediated by the RecQ-like helicase due to unregulated Top3 function. Caspari et al. provide evidence that checkpoint-dependent hyperphosphorylation of $\mathrm{Crb} 2$ is necessary for regulating Top3 activity using a crb2-T215A mutant allele, and they suggest that Cdc2cyclin B regulates Crb2's hyperphosphorylated state by preventing premature dephosphorylation of Crb2.

Recovery, Adaptation, and Apoptosis.

Upon repair of damage, cells can resume cycling. But is the completion of repair sufficient to turn off the checkpoint arrest? Indeed, a few significant pieces of data indicate that the checkpoint cascade must be actively down-regulated. In budding yeast, overexpression of Ddc2 (the ATRIP ortholog) results in an irreversible G2/M arrest after damage (Clerici et al., 2001). This suggests that the ability to shut off the checkpoint response is abrogated in Ddc2-overexpressing cells. Additional evidence that recovery from damage is an active process is supported by the fact that yeast undergo adaptation, a 
process by which they override arrest to resume cell cycle progression despite the presence of unrepaired DNA. The activity of Rad53 and Chk1 must be down-regulated to permit adaptation, indicating that the kinase cascade must be shut off (Pellicioli et al., 2001). This process involves Cdc5, a downstream substrate of Rad53 (Cheng et al., 1998), which may operate in a feedback loop to inactivate the checkpoint cascade (Sanchez et al., 1999). Additionally, the inactivation event may be a regulatory phosphorylation on $\operatorname{Rad} 9$, a necessary protein for Rad53 catalytic autophosphorylation, since in fission yeast $\mathrm{Cdc} 2$ is required to phosphorylate $\mathrm{Crb} 2$, the orthologous $\operatorname{Rad} 9$ homolog, to shut off Chk1 activation and reestablish cell cycle progression (Esashi \& Yanagida, 1999). The clearest example of checkpoint inactivation in mammalian cells involves the p53-inducible protein Wipl. Genotoxic stress leads to p53 activation by p38 phosphorylation, consequently inducing transcription of a number of genes, including Wip1 (Takekawa et al., 2000). Accumulation of the Wip1 phosphatase then inactivates p38, demonstrated both in vivo and in vitro, leading to p53 transcriptional downregulation and a reduced apoptotic response. Attenuation of cell cycle arrest was not specifically examined, but it, too, is predicted to be affected.

In many higher eukaryotes, extensive DNA damage that cannot be repaired during a checkpoint-controlled cell cycle arrest is though to initiate cell death via the apoptotic pathway. That $\mathrm{p} 53$ is a key effector of ATM/ATR protein kinases during checkpoint responses strongly suggests that checkpoint controls and apoptotic controls are coordinated processes within these cells. Mice harboring mutations in p53 or ATM are 
defective in response to DNA damage for both checkpoint activation and apoptosis (reviewed in Khanna \& Jackson, 2001). Also, studies in C. elegans suggest a prominent role in arrest and apoptosis for an orthologous Rad1 sensor protein in germ cells (Gartner et al., 2000). (Curiously, C. elegans somatic cells do not undergo either fate, only their germ cells.) Whether checkpoint protein interaction with damage is somehow central to the switch between arrest and apoptosis or whether the decision lies elsewhere in signal transduction is unknown. Interaction between the human $\operatorname{Rad} 9$ damage sensor protein and BCL apoptotic proteins was recently reported (Komatsu et al., 2000).

\section{Additional Roles of Checkpoint Proteins in DNA Metabolism.}

The checkpoint proteins initially identified as controlling the cell cycle have many additional roles in DNA metabolism, as well. These include transcription of damageresponsive genes and roles in DNA repair (discussed in the "Repair" section), telomere biology, meiotic functions different from those operating in mitotic cells, and control of dNTP pools. Furthermore, proteins that act together in one pathway or response may not act together in a second response, indicating that "complexes" are not necessarily constitutive.

Prominent among the "additional roles" of checkpoint proteins is their part in telomere biosynthesis. In mammals, atm mutants have shorter telomeres, as do budding yeast tel1 mutants. These initial observations led way to the remarkable finding that the fission yeast rad3 and tel1 proteins are required for telomere addition (Matsuura et al., 
1999), followed by similar findings for budding yeast Mecl and Tell proteins (Ritchie et al., 1999). Tell appears to recruit telomerase to sites of novel telomere addition, though exactly how Tell and Mecl do so is not yet known. In budding yeast, Mec1 and Dun1 also effect gene expression of telomere-proximal genes, suggesting some role in chromatin structure (Craven \& Petes, 2000; de le Torre Ruiz \& Lowndes, 2000). An affect on telomere chromatin is also indicated by the finding that in budding yeast some repair and chromatin proteins are re-localized from the telomeres to sites of DNA damage - recruitment that requires checkpoint protein functions $(M e c 1, \operatorname{Rad} 9$, and $\operatorname{Rad} 53$; McAinsh et al., 1999; Mills et al., 1999; Longhese et al., 2000). Yet an additional and, oddly, opposite effect on telomeres is mediated by budding yeast Mec3 and Rad17 proteins; inexplicably, mec3 mutants have shorter telomeres and rad17 mutants longer (Corda et al., 1999; Longhese et al., 2000). Recall that Mecl and Rad17 both act coordinately in cell cycle arrest in what is the equivalent of the 9-1-1 mammalian complex. C. elegans radl mutants also have shorter telomeres (Ahmed \& Hodgkin, 2000), implicating a role for this class of proteins in telomere biosynthesis in many organisms. That checkpoint proteins might interact with telomeres is not unexpected, given that telomeres, by their nature, might resemble a DSB. Hence, checkpoint proteins might be expected to interact with telomeres because they may resemble damage and/or to perhaps aid in their protection.

Study of meiosis has provided yet another fertile ground for discovery of checkpoint protein functions. Checkpoint proteins play many roles in meiosis, including imparting 2 cell cycle delays and regulating partner choice in recombination (Lydall et 
al., 1996; Grushcow et al., 1999; Thompson \& Stahl, 1999). The details of checkpoint protein functions in meiosis are sometimes divergent from their roles in mitosis. For example, Rad9 is not required for meiotic arrest (Lydall et al., 1996), and members of the 9-1-1 complex act at independent steps during meiotic arrest (Thompson \& Stahl, 1999; Hong \& Roeder, 2002). Meiotic studies also reveal the diversity of arrest mechanisms indifferent organisms. Like mammals do for mitotic G2 arrest, budding yeast arrest meiosis (called the pachytene arrest) through inhibitory phosphorylation of $\operatorname{Cdc} 28$, the $S$. cerevisiae ortholog of the Cdc2 protein (Leu \& Roeder, 1999). The mechanisms of meiotic arrest in fission yeast still remain unclear.

Finally, budding yeast checkpoint proteins have other important roles in the regulation of DNA replication independent of cell cycle progression and replication fork stability. Mec1 and Rad53 regulate the synthesis of dNTPs by blocking activity of Smll, an inhibitor of ribonucleotide reductase (Chabes et al., 1999). Mec1 and Rad53 constitutively inhibit Sml1, illustrated by the fact that mecl and rad53 mutants are inviable due to Smll inhibition of the ribonucleotide reductase protein $(\mathrm{Rnrl})$ and that mecl and rad53 mutants become viable when smll is deleted (Zhao et al., 1998). Mecl also appears to regulate replication fork movement in specific "replication slow zones" within the yeast chromosome, and slow replication is again relieved by mutation of $s m l l$ or up-regulation of $R N R I$ (Desany et al., 1998; Cha and Kleckner, submitted). To this end, Mecl may somehow regulate replication fork progression and/or more simply regulate the level of dNTPs. 


\section{Concluding Remarks Regarding Genome Stability.}

The importance of checkpoints in biology, and especially in human cancer, is likely due to their preeminent roles in maintaining genomic stability. Multiple significant links between checkpoint proteins and genome stability have been identified in model organisms, as well as in mammalian cells. In mammals, ATM, ATR, BRCA1, CHK2, and p53 mutants all show instability. For ATM, some of this instability may be due to shortening of telomeres, leading to telomere fusions and breakage-fusion-bridge events (reviewed in Pandita, 2002). However, like most other checkpoints proteins, ATM is likely involved in preserving stability by several mechanisms independent of telomere biology. For example, $\mathrm{ATM}^{-/-}$mutants exhibit elevated mitotic recombination (Kohn et al., 1982). In another example, BRCA1 mutants have partial defects in homologous recombination and transcription-coupled repair (Abbott et al., 1999; Moynahan et al., 1999). As better systems to study instability in mammalian cells are developed, our understanding of these processes will undoubtedly improve.

In budding yeast, the roles of checkpoint genes in genome stability are now receiving extensive analysis. The general mechanisms linking checkpoint protein function and genome stability are slowly emerging, illustrated by four examples discussed below. However, the specific molecular mechanisms of stability are generally unknown along with whether the relevant protein function is in cell cycle progression or in other aspects of DNA metabolism. In the first example, Paulovich et al. (1998) showed that after UV irradiation, checkpoint proteins promote error-prone repair carried 
out by Rev3/Rev7. Whether a cell cycle delay is required for Rev3/7 function or whether checkpoint proteins act more directly, perhaps by recruiting Rev3/7 to UV lesions is unknown. Second, checkpoint genes seem to promote allelic recombination (Bashkirov et al., 2000). Defects in this process lead to an increased frequency of ectopic recombination in meiotic (Grushcow et al., 1999) and mitotic cells (Fasullo et al., 1998, plus defects in intergenic mitotic recombination (Bashkirov et al., 2000). Third, rad9 and other checkpoint mutants spontaneously lose chromosomes (Weinert \& Hartwell, 1990; Klein, 2001). One mechanism of loss was, until recently, thought to involve mitosis of a cell with a chromosome having an unrepaired DSB. Surprisingly, one recent study indicates that a cell completely defective for the G2/M checkpoint (a dunl chkl double mutant) undergoes no detectable spontaneous chromosome loss (Klein, 2001). Therefore, chromosome loss in rad9 mutants is likely due to a defect in some other cellular activity.

Finally, and in our view most significantly, checkpoint proteins may play key roles in maintaining stalled replication forks, because when defective the collapsed forks lead either to cell death or to genomic rearrangements. Myung and Kolodner (2002) have carried out an extensive analysis of the effects of checkpoint mutants on a spectrum of chromosome arrangements they call gross chromosome rearrangements, or GCR. They suggest that GCRs in yeast structurally resemble those rearrangements seen in cancer cells. By surveying checkpoint mutants for GCR they found that, in general, the proteins regulating S-phase responses had larger roles in maintaining stability, but with numerous 
exceptions and enigmatic observations. (For example, Dun1 and Mec3 have significant roles in stability, though whether it is their S-phase roles that are involved is unclear; their roles in telomere metabolism seems just as likely.) Defects in both Mecl and Tel1, the two ATR- and ATM-like genes, respectively, lead to a 10,000-fold increase in instability. But which of their many functions contributes to stability remains uncertain; they mediate essentially all responses to DNA damage, are required for telomere synthesis, and play roles in DNA replication. Defects in Pds1 also strongly affect stability, implicating the role of Pds 1 in sister chromatin cohesion rather than its role in G2/M arrest since chkl mutants having a similar arrest defect demonstrate no instability. Linking complex replication fork biology, checkpoint protein function, and genome stability remains a major challenge, but one we anticipate is fundamental to understanding cancer etiology and treatment. 


\section{CHAPTER 2. ANALYSIS OF RAD9 FUNCTIONS BY IDENTIFYING SEPARATION-OF-FUNCTION MUTATIONS}

Statement by author

I performed all experiments described in this chapter except those noted as done in collaboration with David Lydall.

\section{SUMMARY}

Though the Saccharomyces cerevisiae RAD 9 checkpoint gene was first identified and cloned 15 years ago, a detailed understanding of $R A D 9$ function remains to be elucidated. rad 9 cells lack the ability to arrest in response to DNA damage and also demonstrate marked genomic instability, but the role of $R A D 9$ in repairing or processing such damage and its contribution to the instability phenomenon is unclear. Various experimental evidence suggest that the $\operatorname{Rad} 9$ protein performs at least two functions: one mediating cell cycle arrest and repair in response to DNA damage and another preventing degradation of damaged DNA by the $\operatorname{Rad} 24 / \operatorname{Rad} 17 / \mathrm{Mec} 3 / \mathrm{Ddc} 1$ epistasis group. While the arrest response has been extensively studied, questions regarding this function still remain. Moreover, little is know about the apparent role of Rad9 in inhibiting DNA degradation. This function may be related to the ability of Rad9 to prevent genomic instability and/or may constitute a damage-processing function with implications in DNA repair. Based on these observations, I pursued experiments aimed at identifying rad 9 separation-of-function mutations to enable us to better understand $\operatorname{Rad} 9$ functions, its interactions with other checkpoint and repair proteins, and its role in preventing genomic instability. 


\section{INTRODUCTION}

DNA holds a precious store of information from which all life is derived. Being constantly subject to the bombardment of exogenous and endogenous DNA damage, the need to protect DNA becomes a matter of life and death. To enhance cell survival, selective pressure has provided the impetus for the evolution of controls that carefully monitor the state and care of DNA. Checkpoints comprise one such control.

Checkpoints serve as a surveillance mechanism for the cell, specifically recognizing damaged or incompletely replicated DNA. While non-essential for cell cycle events, checkpoints enforce the correct order of events to ensure that damaged or incompletely replicated DNA gets repaired before passage to the next phase where damage may be propagated (Weinert, 1998). Loss of checkpoint controls is often catastrophic for the cell or organism, since these proteins function to increase cell survival by recognizing DNA damage and subsequently allowing time for DNA repair.

The dependence of cellular fidelity on checkpoint controls becomes apparent when one considers the disasters that ensue in their absence. When checkpoints are inoperable, DNA damage can accumulate, thereby leading to a loss of essential genes and predisposing cells to genomic instability (Paulovich et al., 1997). For a unicellular organism in such instances, the result is typically death. For humans, the consequences can be equally as dire, as loss of checkpoint proteins - such as p53 and ATM - can lead to unregulated cell growth of DNA-damaged cells that ultimately develops into cancer or other debilitating diseases (Weinert, 1997).

Now widely recognized for their significance in preventing the propagation of DNA damage, checkpoints are studied in a variety of organisms including budding and fission yeasts, flies, worms, and mammalian cells. These studies reveal profound conservation of the types of checkpoint responses and also the genes that regulate such 
processes. Such similarities provide hope that knowledge gained by studying checkpoints in yeast, which are more tractable to genetic manipulation and experimentation, will enable us to better understand checkpoint controls in humans. Ultimately, this may allow for the development of strategies that employ discriminate killing of cancer cells by selectively blocking residual checkpoint functions, thereby rendering cells more sensitive to DNA damage that then leads to their death (Paulovich et al., 1997).

Since their initial discovery, research has furthered our understanding of the critical roles checkpoint proteins play and how they carry out these functions. Checkpoints that recognize DNA damage or DNA replication defects can induce cell cycle arrest in $\mathrm{G}_{1}$ and $\mathrm{G}_{2}$ (Weinert and Hartwell, 1988; Siede et al., 1994), cause slowing of DNA replication during S-phase (Paulovich and Hartwell, 1995), and increase transcription of a regulon of repair genes (Aboussekhra et al., 1998), all of which facilitate DNA repair processes. The process of cell cycle arrest by checkpoints most likely occurs in three steps. First, sensor proteins detect DNA damage. Upon so doing, they, secondly, transduce a signal to proteins that, ultimately, modify target molecules to induce arrest. Checkpoint proteins that function in each step of this process have been identified and may be classified into groups corresponding to sensors, signalers, and targets (refer to Table 1-1 on page 20).

Sensor proteins are inferred to recognize aberrant DNA structures - likely to include single-stranded DNA (ssDNA), double-strand DNA breaks, faulty replication forks, and aberrant chromatin structure. One group of checkpoint proteins known to localize at sites of damage includes $\operatorname{Rad} 24, \operatorname{Rad} 17, \mathrm{Mec} 3$, and Ddc1, hereafter referred to as Rad24 et al (Kondo et al., 2001; Melo et al., 2001). Distinct from the $\operatorname{Rad} 24$ et al. group is another set of proteins, Mecl and Ddc2, that also localizes to damaged DNA (Rouse \& Jackson, 2002; Kondo et al., 2001; Melo et al., 2001). Whether these proteins are the primary sensors that directly recognize DNA damage of whether they are 
secondary sensors that associate with other proteins localized at sites of damage is unknown.

Rad9 was previously thought to also be a checkpoint protein sensor, namely because earlier genetic work demonstrated that $R A D 9$ exists in an epistasis group separate from that of the RAD24 et al. group of genes. Subsequent work, however, disfavors the model in which Rad9 associates with DNA damage as a sensor, but rather lends support to a model wherein Rad9 actually serves to transduce the DNA damage checkpoint signal after phosphorylation and activation by $\mathrm{Mecl}$.

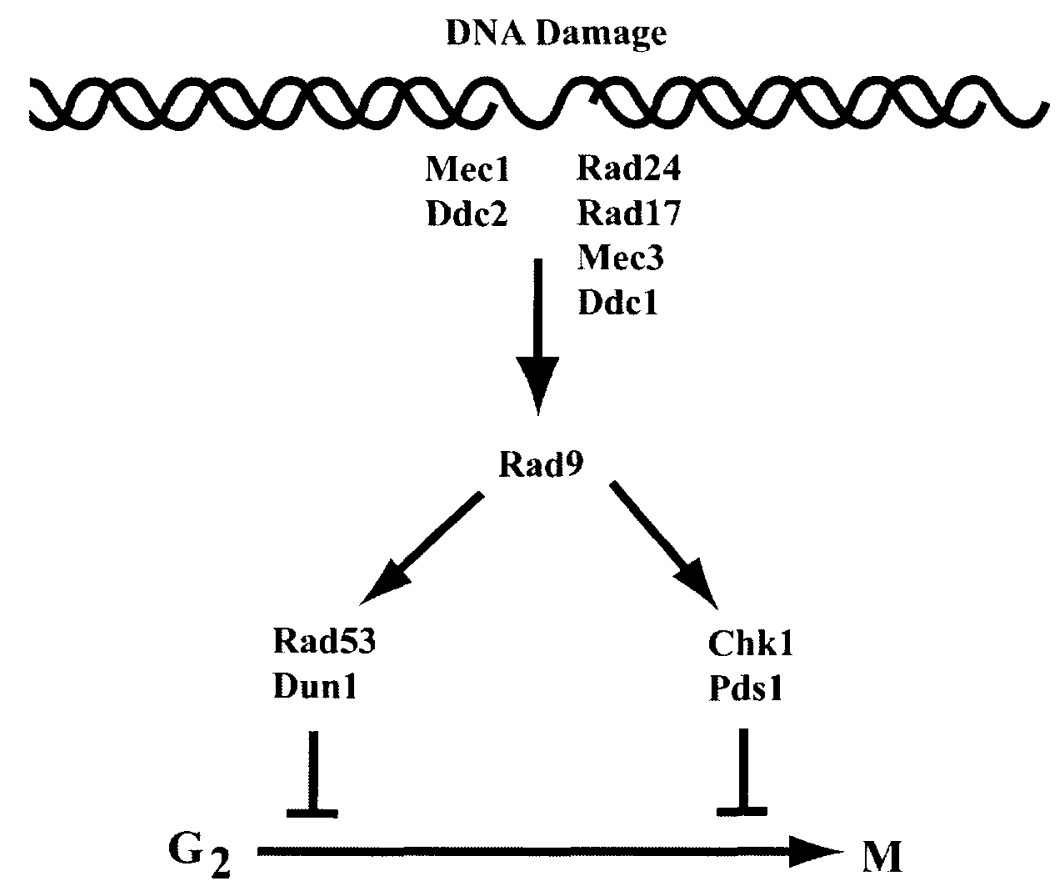

Figure 2-1. A current model of the checkpoint cascade leading to $\mathrm{G}_{2} / \mathrm{M}$ arrest. Sensor proteins, including $\mathrm{Mec1} / \mathrm{Ddc} 1$ and the $\mathrm{Rad} 24 / \mathrm{Rad} 17 / \mathrm{Mec} 3 / \mathrm{Ddc} 1$ epistasis groups, detect DNA damage. Mec1 phosphorylates $\operatorname{Rad} 9$ to transduce the damage signal to the downstream protein kinases, Rad53, Dun1, and Chk1. These signaler proteins then modify target proteins, including Pds1, to induce arrest at the $\mathrm{G}_{2} / \mathrm{M}$-phase transition allowing time for DNA repair. 
$\mathrm{Mecl}$ is the central regulator of checkpoint responses as it promotes arrest after DNA damage at all cell cycle phases (Figure 2-1; Weinert, 1998). In addition to its essential function in regulating dNTP levels within the cell, Mecl appears to control two pathways that lead to arrest when DNA damage is present (Cohen-Fix and Koshland, 1997; Gardner et al., 1999; Sanchez et al., 1999). One pathway includes Rad53 and Dun1, signaler protein kinases that are both necessary to induce transcription of repair genes and to prevent activation of the mitotic exit pathway in the presence of DNA damage. The second parallel Mecl-dependent pathway leads to activation of the Chk1 protein kinase, which then prevents anaphase entry by controlling phosphorylation and abundance of the target protein Pds1 (Sanchez et al., 1999). Phosphorylation of Pds1 blocks its degradation, which is necessary for the initiation of anaphase.

A current model posits that upon activation by Mec1, Rad9 dimerizes via its only

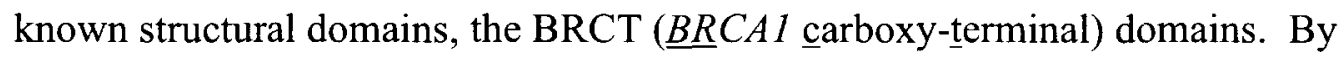
dimerizing, two Rad53 molecules, which are known to bind phosphorylated Rad9, are brought in close enough proximity to one another that they can then autophosphorylate the other, thereby potentiating the checkpoint cascade (Gilbert et al., 2001). Chk1 also becomes activated after Rad9 phosphorylation, though the mechanism of this activation event is not well understood.

While great strides have been made in the advancement of knowledge regarding checkpoint responses and some of the mechanisms involved, many questions yet remain unanswered. We are still far from understanding how the known checkpoint proteins function, including how they recognize DNA damage, exactly how the arrest signal is relayed through the checkpoint cascade, and what actions the checkpoint proteins take to process, prevent, or repair damage. Add to that the yet unidentified checkpoint proteins awaiting discovery, subsequent characterization, and placement within the checkpoint cascade. 
The opposing role of checkpoint proteins in ssDNA formation.

One such checkpoint process that is not well understood is that involving the opposing actions of the RAD 9 and $R A D 24$ et al. genes in generating ssDNA. While the $R A D 9$ and $R A D 24$ et al. epistasis groups share a similar role in initiating cell cycle arrest in the presence of DNA damage and promoting the repair of such damage, they have opposing roles in generating ssDNA that may be indicative of DNA damage processing. In the presence of the temperature-sensitive $c d c 13-1$ mutation that creates damage at chromosome ends, $\operatorname{rad} 9$ and $\mathrm{rad} 24$ mutants are equally checkpoint-defective, but the cdc13-1 rad 9 mutant loses viability much more rapidly than does a $c d c 13-1 \mathrm{rad} 24$ mutant. Additionally, $c d c 13-1$ rad 9 cells accumulate ssDNA damage at chromosome ends much faster and to a much greater extent (3-fold higher) than $c d c 13-1$ rad24 cells (Lydall \& Weinert, 1995; Garvik et al., 1995). Especially important is the result that a cdc13-1 rad9 rad24 triple mutant shows little accumulation of ssDNA and is as viable as wild-type cells (Lydall \& Weinert, 1995). This additionally demonstrates that $R A D 9$ and the RAD24 et al. proteins reside in different epistasis groups and that they mediate different functions in regard to ssDNA formation at chromosome ends. Such results led to the currently-held model that damage incurred in $c d c 13-1$ mutants is processed into large single-stranded regions by RAD24 et al., which act to regulate the ExoI exonuclease and another yet unidentified exonuclease (Maringele \& Lydall, 2002). This ssDNA processing is antagonized by functional $R A D 9$. Thus, $c d c 13-1 \mathrm{rad} 9$ mutants fail to block RAD24 et al. nucleolytic degradation and demonstrate an increased accumulation of ssDNA at chromosome ends, whereas $c d c 13-1 \mathrm{rad} 24$ and $c d c 13-1 \mathrm{rad} 9 \mathrm{rad} 24$ mutants are defective for DNA degradation and show little increased incidence of single-stranded telomeric DNA. Furthermore, these results suggest that ssDNA may be the specific signal for the arrest checkpoint (Garvik et al., 1995).

Why would proteins known to prevent DNA damage actually create more damage on their own? To expound upon the model, it may be that some damage processing must 
occur, namely by the $\operatorname{Rad} 24$ et al. group of proteins, to produce a sufficient ssDNA signal that other checkpoint proteins (e.g., Mecl) recognize to then induce arrest. Once the correct damage signal is recognized, only then might Rad9 act to prevent further damage or initiate repair by inhibiting degradation by the $\operatorname{Rad} 24$ group. Therefore, according to this model, at least two roles for Rad9 are established - one that causes arrest in response to damage and one that blocks additional damage processing by $\operatorname{Rad} 24$ et al.

The goal of this portion of my dissertation project was to better understand Rad9 function by delineating its role in inhibiting degradation of damaged DNA and its role in the arrest response. Questions I sought to address included: 1) Is Rad9 inhibition of degradation related to its arrest function?; 2) What is the nature of $\operatorname{Rad} 9$ function that prevents degradation of damaged DNA?; in other words, is Rad9's role direct in that it alone can prevent degradation by the $\operatorname{Rad} 24$ group of proteins, or is it indirect, whereby Rad9 mediates the block using other proteins that associate with damaged DNA?;3) Are there novel Rad9 protein interactions that relate to the inhibition-of-degradation function, and, if so, do these proteins play any role in the arrest phenotype?; and, 4) How do the inhibition-of-degradation and arrest functions contribute to Rad9's prevention of genomic instability?

To better understand the role Rad9 plays in mediating both cell cycle arrest in response to DNA damage and its inhibition of DNA degradation by Rad24 et al., I performed a screen of mutated rad9 alleles to identify those deficient in one of these two known functions of Rad9. I was particularly interested in finding mutants that were defective in inhibiting DNA degradation but proficient for causing cell cycle arrest in the presence of damage in order to: 1) determine if the two roles are indeed separable, that is, is the ability to arrest the cell cycle contingent upon the ability to block degradation, and, 2) use such a mutant in a suppressor screen for genes that restore the ability to block or prevent DNA degradation in order to identify other genetic components of this pathway. 


\section{RESULTS}

Creation of rad9 separation-of-function mutants.

To create mutated rad 9 alleles, I employed the method of pentapeptide insertional mutagenesis. This method was selected because the procedure is simple, it creates a large bank of mutants for screening, and single mutations are introduced randomly within the gene sequence.

Pentapeptide insertional mutagenesis utilizes the random insertion of the TN4430 transposon into a plasmid containing a gene of interest (Hallet et al., 1997; Hayes et al., 1997). The RAD9 sequence was cloned into the pRS416 (centromeric, URA3-marked) vector. This plasmid was then subject to TN4430 transposon insertion. A library of plasmids containing the trasnposon insertion within the RAD9 sequence was identified and obtained by restriction digest (Fig. 2-2). The Kpn1 restriction endonuclease was then used on this plasmid library to excise the bulk of the transposon, leaving behind an inframe 15-bp insertion within the target gene. The transposon insertion scheme was designed so that the majority of in-frame 15-bp insertions results in missense mutations at the protein level and rarely results in introduction of a STOP codon within the nucleotide sequence. Thus, a random distribution of pentapeptide insertions within the Rad 9 protein sequence was created. Because the mutated sequences were all plasmid-based, the mutations could be screened in the yeast background of our choosing. 
L1

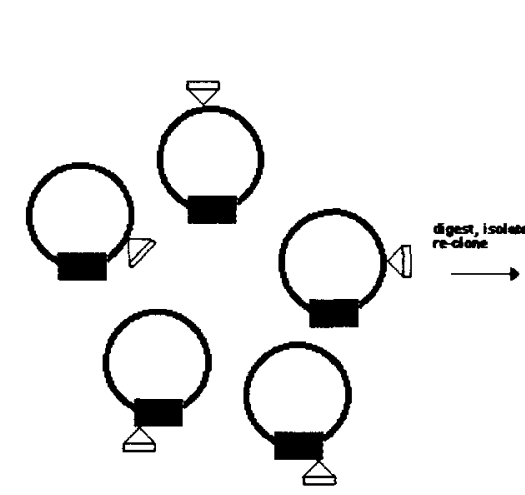

transposon insertion into vector containing gene of interest
L2

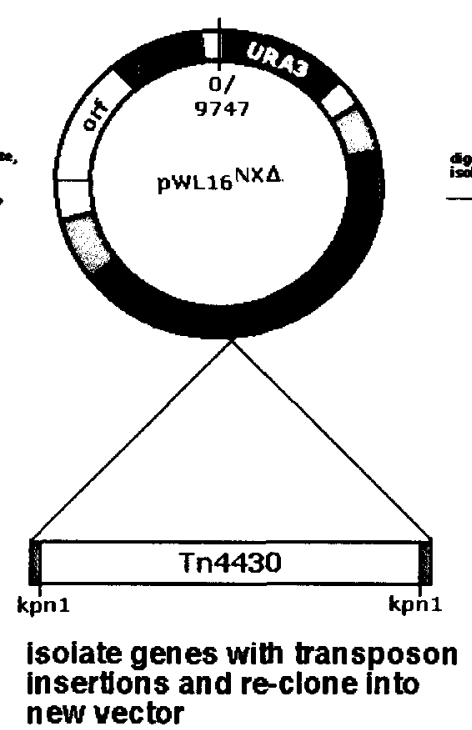

$\mathbf{L 3}$

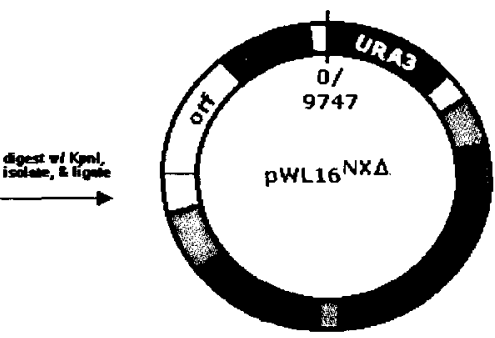

excise transposon by Kpnl digestion and re-ligate plasmid to generate pentapeptide insertions

Figure 2-2. The pentapeptide insertional mutagenesis scheme. The transposon, in yellow, randomly inserts within a plasmid containing the $R A D 9$ sequence, shown in purple. This creates a library of plasmids (L1) containing the transposon insertion, either within the $R A D 9$ sequence or within the vector backbone. Restriction digests identify those plasmids containing the transposon solely within the RAD9 sequence. This DNA is isolated and ligated into a new vector to constitute the second library (L2). These plasmids are then subject to KpnI digestion to excise the bulk of the transposon, fragment isolation, and religation to generate the third and final library (L3). This library consists of plasmids containing a random distribution of 15-bp insertions within the RAD9 sequence. Figure from Nicole Washington.

While conducting the screen, I crudely determined the randomness of the transposon insertions within the $R A D 9$ sequence. $R A D 9$ sequences containing 15 -bp insertions were isolated by restriction digest and gel purification from the final library used for screening. This purified $R A D 9$ DNA fragment was then cut with the restriction endonuclease KpnI, which cuts within the 15-bp insertion. Running the KpnI-cut DNA on an agarose gel produced a smear of sizes $<100$ bases to $5 \mathrm{~kb}$; the size of the full-length 
excised $R A D 9$ sequence is $5 \mathrm{~kb}$. The smear was not of uniform intensity, but, rather, several bands were visible above the background smear all along the length of digested DNA. These bands are assumed to correspond to $R A D 9$ sequence with the transposon inserted at or very near the same location within the primary sequence. This could indicate that either there are hot spots within the $R A D 9$ sequence that are more amenable to transposon insertion or that some mutagenized rad9 sequences were selectively amplified over others during the various steps of transformation and isolation from bacteria. Regardless, the smeariness of the DNA digest signifies that the pentapeptide transposon insertion method generates fairly random mutations, though I realized some specific mutated rad 9 sequences may actually be screened several times.

Screening rad9 separation-of-function mutants.

Plasmids containing mutagenized rad 9 were transformed into a strain with a cdc13-1 background to facilitate the screening process. CDC13 encodes a protein known to associate with and protect telomere sequences (Nugent et al., 1996). When the temperature-sensitive $c d c 13-1$ strain is shifted to the restrictive temperature $\left(>28^{\circ} \mathrm{C}\right)$, ssDNA begins to accumulate at DNA ends and cells exhibit a checkpoint-dependent arrest at the $\mathrm{G}_{2} / \mathrm{M}$ phase transition (Garvik et al., 1995). Checkpoint-deficient cells, such as a rad9 mutant, proceed past the $\mathrm{G}_{2} / \mathrm{M}$ transition unchecked while degrading chromosome ends and continue cell division until they finally lose viability.

Two primary screens were performed to assess the arrest and inhibition-ofdegradation functions of the mutated rad9 alleles (Lydall \& Weinert, 1995). The primary arrest assay directly determined arrest proficiency by the following scheme: midlogarithmic cells grown at the permissive temperature were plated and shifted to the restrictive temperature $\left(36^{\circ} \mathrm{C}\right)$ for 6 hours. Cells were then examined by microscopy and scored as being either arrest-proficient (having arrested as 2- or 4-budded cells) or as being arrest-defective, indicated by microcolony formation ( $\geq 5$ cells). The primary screen for inhibition-of-degradation was indirectly assessed by cells death, as rapid DNA 
degradation is thought to cause rapid death, presumably due to loss of essential genes. Notably, $c d c 13-1$ mutants are reported to accumulate ssDNA up to $20 \mathrm{~kb}$ from the ends of telomeres (Garvik et al., 1995). In this assay, mid-logarithmic cells were plated and incubated at the restrictive temperature for 4 hours to induce $c d c 13-1$ inactivation and DNA damage. The plates were then transferred back to the permissive temperature $\left(23^{\circ} \mathrm{C}\right)$ for 16 hours to allow cells to recover and repair DNA damage. Cells were then again incubated at the restrictive temperature for 4 hours and again shifted back to the permissive temperature for 16-40 hours. The double shift serves to square the difference between those cells that are able to survive and those that are not, allowing for better discrimination of those cells that can prevent DNA degradation by mass screening. After this primary screening, those mutations that separated function - cells containing a rad 9 mutation that were proficient for one function and deficient in the other - were then isolated for further testing.

At the same time as the arrest and inhibition-of-degradation screens were being performed, I also analyzed the UV and methyl methane sulfonate (MMS) sensitivities of the transposon-mutated rad 9 alleles. For the UV assay, cells were plated and then exposed to $90 \mathrm{~J} / \mathrm{m}^{2} \mathrm{UV}$ irradiation. Cells were then allowed to grow for 12-16 hours at the permissive temperature, and subsequently scored for their UV sensitivity based on the robustness of growth. MMS is an alkylating DNA damaging agent that causes singleand double-strand breaks upon repair. In assessing MMS sensitivity, cells were plated to media containing $0.01 \% \mathrm{MMS}$, incubated overnight at the permissive temperature, and scored based on growth the following day.

In total, 800 mutated rad 9 alleles were assessed for arrest proficiency, inhibitionof-degradation function, UV sensitivity, and MMS sensitivity according to the primary screening protocols. Of these 800 mutants, 194 appeared to be putative separation-offunction mutants when comparing arrest and inhibition-of-degradation phenotypes after the first pass of screening. More specifically, 119 were classified as Arrest ${ }^{+}$inhibition- 
arrest $^{-}$Inhibition-of-Degradation $^{+}$

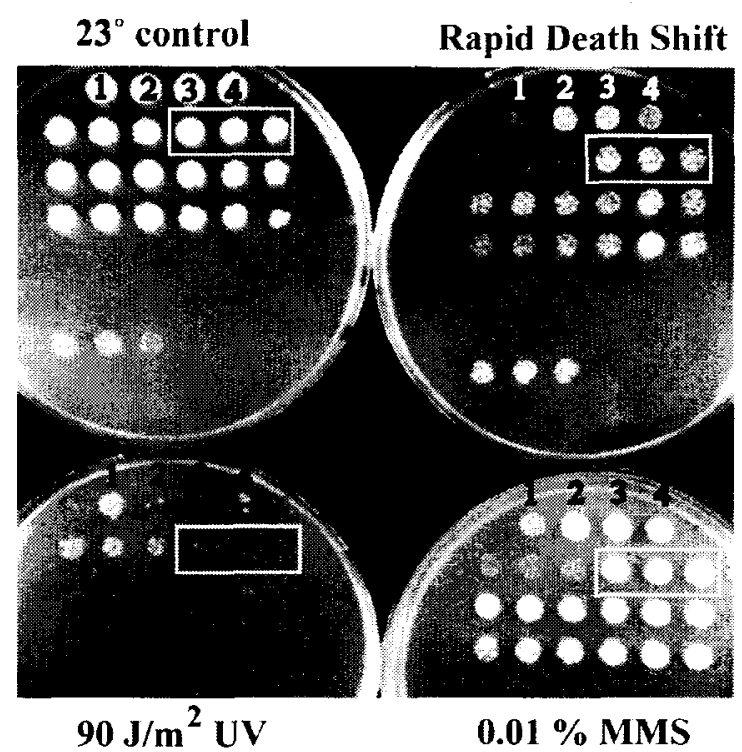

Arrest $^{+}$inhibition-of-degradation

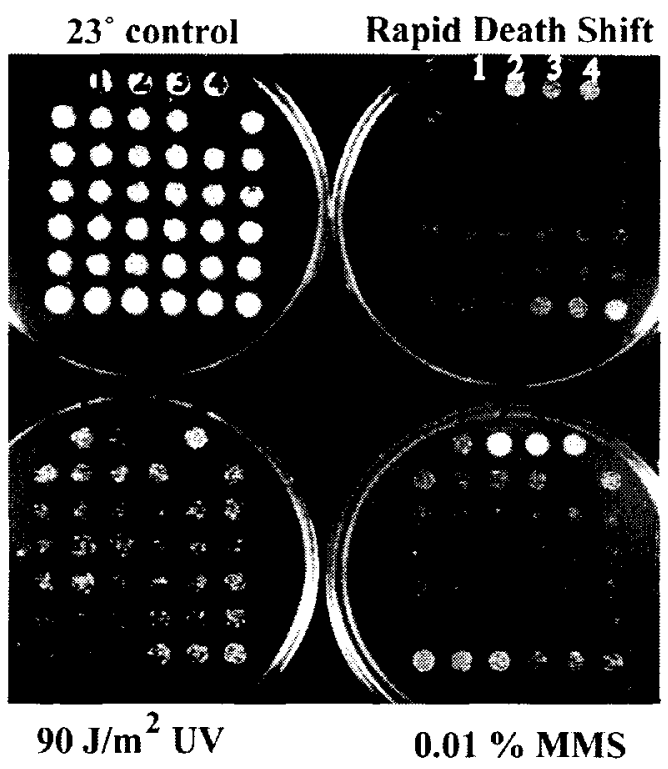

Figure 2-3. Phenotypic analysis of putative rad9 separation-of-function mutants in the primary screen. Mid-logarithmic cells were spotted onto solid media and subjected to the assaying conditions described previously. Each panel shows a subset of strains containing mutated rad9 alleles that were untreated (top left), subjected to the rapid death growth conditions at restrictive and permissive temperatures (top right), subjected to assault with $90 \mathrm{~J} / \mathrm{m}^{2} \mathrm{UV}$ irradiation (bottom left), and subjected to growth on media containing $0.01 \%$ MMS (bottom right). The top row of each plate depicts growth of the four control strains - $\operatorname{rad} 9(\# 1), \operatorname{rad} 24(\# 2), \operatorname{rad} 9 \operatorname{rad} 24$ (\#3), and wild-type (\#4) - used for comparison with the mutant rad9 strains. Below these controls, three individual colonies from mutant plasmid retransformations were grown, so that each row shows the phenotypes of two rad9 mutant strains. As an example, the white box in the left panel surrounds one rad 9 mutant strain tested in triplicate. This mutant shows resistance to DNA degradation (rapid death plate) and MMS damage but is sensitive to UV damage.

of-degradation, while 75 exhibited an arrest ${ }^{-}$Inhibition-of-Degradation ${ }^{+}$phenotype. Because I was generous in their classification as having a separation-of-function phenotype, these 194 mutants were put through a second pass of primary screening to eliminate false positives and verify phenotypes. Only 22 of these 194 mutants convincingly separated function by phenotype in the second primary screening assessment, 13 being Arrest ${ }^{+}$inhibition-of-degradation ${ }^{-}$and 9 being arrest ${ }^{-}$Inhibition-ofDegradation $^{+}$. Figure 2-3 shows a subset of these 22 mutants after the second pass through the primary screen. 
Plasmids containing the mutated rad9 alleles for these 22 putative separation-offunction mutants were isolated from yeast, transformed into bacteria for amplification, and retransformed back into yeast to test plasmid-linkage of the phenotypes. Upon retransformation into yeast, only 16 of the strains exhibited the separation-of-function phenotypes that characterized these mutants in the primary screen, indicating that 6 of the original 22 mutants characterized had some endogenous background mutation that created the separation-of-function phenotype and that it was not due to rad 9 mutation. This result is not unexpected given that the process of transforming plasmids into yeast cells is, in and of itself, potentially mutagenic. Botstein and Shortle (1984) estimated that plasmid transformation induces a significant rate of background mutations.

Quantitative analysis of putative separation-of-function mutants.

More thorough and comprehensive secondary screening of the 16 plasmid-linked putative separation-of-function mutants from the primary screen was carried out to quantitatively assess the initial phenotypes witnessed. Arrest proficiency was directly determined by a liquid culture assay. Mid-logarithmic cycling cells were synchronized in the $G_{1}$ phase with $\alpha$-factor at the permissive temperature. The $\alpha$-factor was removed and cells were released to begin cycling at the restrictive temperature of $36^{\circ} \mathrm{C}$ to induce cdc13-1 damage. Cells were fixed at various time points for DAPI staining of nuclei to determine cell cycle position. The inhibition-of-degradation phenotype was measured by synchronizing cells in $\mathrm{G}_{1}$ with $\alpha$-factor, followed by cell cycle release at the restrictive temperature. A low density of cycling cells was plated to solid media at various time points and grown overnight at the permissive temperature. Cells were then scored the following day by comparing the number of cells that did not grow (dead cells, with up to 16 cells in the dead colony) versus living cells that form microcolonies. UV sensitivity was determined by plating a low density of mid-logarithmic cells to solid media, subjecting to UV irradiation, growing overnight at the permissive temperature, and then scoring the following day by counting dead cells versus microcolonies. 


\begin{tabular}{|c|c|c|c|c|c|}
\hline \multirow[b]{2}{*}{$\begin{array}{l}\text { Mutant } \\
\text { allele }\end{array}$} & \multicolumn{3}{|c|}{ Phenotypes } & \multirow[b]{2}{*}{$\begin{array}{c}\text { Site of } \\
\text { Mutation } \\
(R A D g= \\
3930 \text { bases })\end{array}$} & \multirow[b]{2}{*}{$\begin{array}{c}\text { Predicted Effect of } \\
\text { Mutation }\end{array}$} \\
\hline & Arrest & $\begin{array}{l}\text { Inhibition- } \\
\text { of- } \\
\text { Degradation }\end{array}$ & $\begin{array}{c}\text { UV } \\
\text { Resistance }\end{array}$ & & \\
\hline $3-E 5$ & + & $-1+$ & + & At $3.6 \mathrm{~kb}$ & $\begin{array}{l}\text { Abrogates BRCT } \\
\text { domain functions }\end{array}$ \\
\hline $15-\mathrm{C} 2$ & + & + & $-/+$ & At $1.25 \mathrm{~kb}$ & $\begin{array}{l}\text { Affects } \\
\text { phosphorylation sites } \\
\text { recognized by Rad53 }\end{array}$ \\
\hline $16-\mathrm{C} 3$ & + & + & $+1-$ & At $0.6 \mathrm{~kb}$ & Unpredicted Effect \\
\hline $16-\mathrm{C} 5$ & + & $-/+$ & + & At $3.6 \mathrm{~kb}$ & $\begin{array}{l}\text { Abrogates BRCT } \\
\text { domain functions }\end{array}$ \\
\hline $17-\mathrm{F} 5$ & + & $-1+$ & + & At $2.1 \mathrm{~kb}$ & Unpredicted Effect \\
\hline $18-\mathrm{D} 3$ & + & + & + & $\begin{array}{l}\text { Upstream } \\
\text { of Start }\end{array}$ & No Effect \\
\hline 18-E6 & + & $-1+$ & + & $\begin{array}{l}\text { Upstream } \\
\text { of Start }\end{array}$ & $\begin{array}{l}\text { May affect protein } \\
\text { expression levels }\end{array}$ \\
\hline
\end{tabular}

Table 2-1. Comparison of phenotypes of putative separation-of-function mutants. For the various phenotypes analyzed, "+" indicates wild-type growth, "+/-" indicates hypomorphic growth compared to wild-type, and "_“ indicates growth comparable to a rad9 null strain.

Out of this set of 16 mutants, 7 were selected for additional characterization based on their promising separation-of-function phenotypes. The mutated rad 9 sequences were excised from the CEN vector (pRS416), used during the primary and secondary screens, and ligated into an integrating vector (pRS406) to facilitate integration of the mutated sequences into the yeast genome. By cutting with the restriction enzyme Ncol, the rad 9 sequences were targeted to the ura3 locus for integration to create ura3::URA3-rad $9^{\text {mut }}$ within the genome of a $\mathrm{rad} 9^{-} \mathrm{cdc13-1}$ strain (DLY413). Quantitative arrest, inhibitionof-degradation, and UV assays were then repeated with these stably-integrated mutants. 
Table 2-1 summarizes the phenotypes for each of these mutant alleles and the relative sites of 15-bp insertion based upon restriction digest analysis of the various rad 9 sequences.

Two mutants, 3-E5 and 16-C5, produced a fairly robust separation-of-function phenotype above that of other mutants, demonstrating a wild-type cell cycle arrest response in the presence of $c d c 13-1$ damage, wild-type levels of UV resistance, and a hypomorphic inhibition-of-degradation phenotype. Figure 2-4 shows the quantitative phenotypes of the two mutants in comparison with one another and against the control wild-type and null strains. When sequenced to determine the site of the $15-\mathrm{bp}$ insertion, I found that both mutated alleles had the same insertion between bases 3599 and 3600 
a) 100

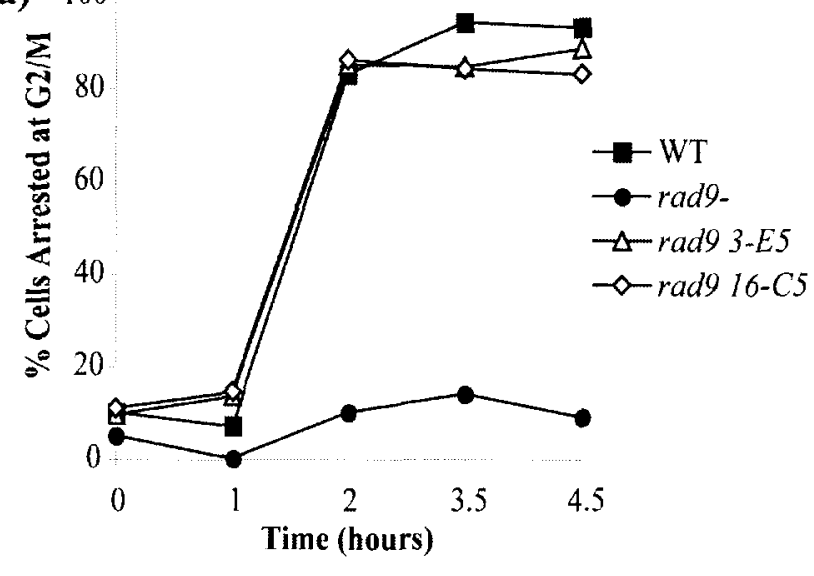

c)

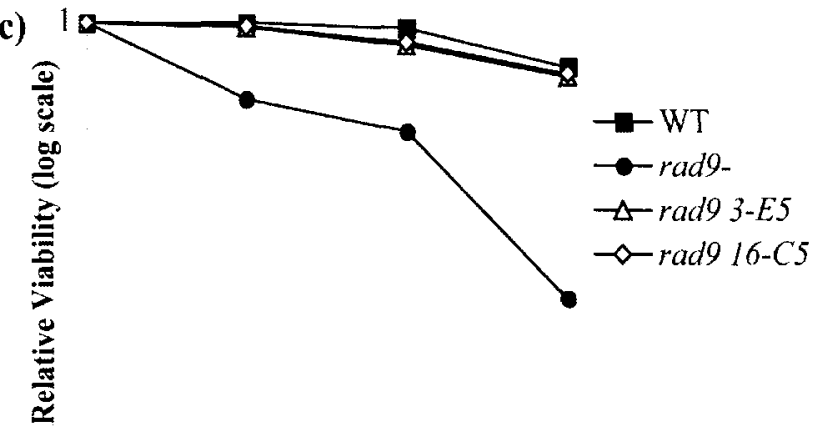

b)

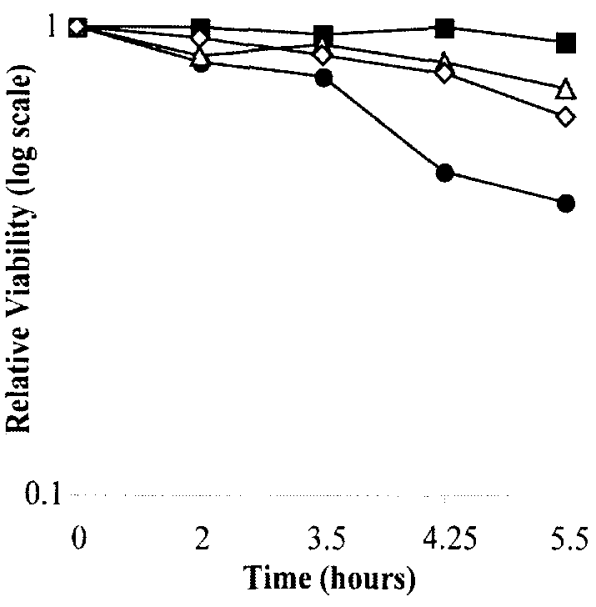

0.1

$\begin{array}{llll}0 & 20 & 40 & 60\end{array}$

UV Dosage (J/M $\left.\mathbf{M}^{2}\right)$

Figure 2-4. Comparison of rad9 3-E5 and rad9 16-C5 phenotypes. (a) For the arrest assay, mid-logarithmic cells were synchronized in $\mathrm{G}_{1}$ with $\alpha$-factor at $23^{\circ} \mathrm{C}$. Cells were then released at the restrictive temperature $\left(36^{\circ} \mathrm{C}\right)$ and fixed at various time points. Cell nuclei were stained with DAPI and analyzed by fluorescent microscopy for cell cycle position. (b) For the inhibition-of-degradation assay, mid-logarithmic cells were synchronized in $\mathrm{G}_{1}$ with $\alpha$-factor at $23^{\circ} \mathrm{C}$. Cells were then released at the restrictive temperature $\left(36^{\circ} \mathrm{C}\right)$ and plated to solid media at various time points. Cells were grown at $23^{\circ} \mathrm{C}$ overnight and scored the following day for microcolony formation. (c) For the UV sensitivity assay, mid-logarithmic cells were plated to solid media and treated with various doses of UV irradiation. Cells were then grown overnight at $23^{\circ} \mathrm{C}$ and scored the following day for microcolony formation. 
within the $R A D 9$ sequence. This creates a 5 amino acid insertion at position 1200 within the Rad9 protein sequence, located within the second BRCT domain (Figure 2-5). This mutated allele was renamed rad9-10 to be consistent with current nomenclature.

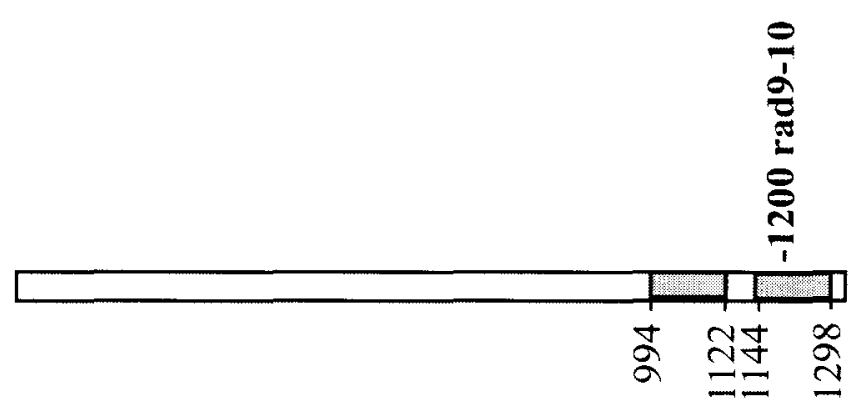

BRCT Domains

Figure 2-5. A map of the rad9-10 mutant. The diagram shows the site of the pentapeptide insertion in the rad9-10 mutant and the position of the BRCT domains. Numbers above indicate amino acid positions.

Rad9's BRCT domains are known to be necessary for facilitating $\operatorname{Rad} 9$ dimerization for function in response to DNA damage (see Chapter 3). One might predict that the rad9-10 insertion in some way abrogates Rad9 dimerization via the BRCT domains. However, because this mutant is proficient for arrest and is UV-resistant, functions that also require Rad9 dimerization, some additional explanation for this mutant is necessary.

One explanation may be that perhaps the insertion is abrogating some unknown Rad9 function in inhibiting DNA degradation, for example, dimerization with another protein that physically blocks degradation mediated by Rad24 et al. Another explanation may depend on levels of protein activity. The inhibition-of-degradation phenotype may 
require a high level of functional $R A D 9$ activity. The rad9-10 mutation, then, may lessen the activity of the protein product to just below the threshold level needed to block DNA degradation. In contrast, the cell cycle arrest and DNA damage repair signals that proceed through Rad9 may be amplified at later points within the checkpoint cascade. The protein kinases Rad53 and Chk1 are inferred to amplify the checkpoint signal; one active kinase molecule can phosphorylate numerous target molecules provided that they remain activated. Thus, arrest and repair might be somewhat less sensitive to attenuated Rad9 activity, as may be the case with the mutant rad9-10 protein. The inhibition-ofdegradation phenotype, on the other hand, may be more sensitive to Rad9 activity if it operates by a mechanism that is not subject to amplification. If $\operatorname{Rad} 9$ and/or closely associated proteins are directly acting to physically block degradation, then one or more blocking proteins may be needed to impede exonucleases at each site of incurred damage. In this case, a mutant like rad9-10 may be defective enough in function to weaken the inhibition-of-degradation of phenotype, while still being proficient enough for the arrest and repair functions.

Not incongruent with this hypothesis is the possibility that the rad9-10 allele may contain a temperature-sensitive mutation, whereupon a shift up to higher temperatures affects the wild-type function of the protein to impact the inhibition-of-degradation phenotype but not the arrest phenotype, which was also measured at elevated temperatures. Because rad9-10 was previously determined to be UV-resistant when assayed at $23^{\circ} \mathrm{C}$, the $\mathrm{CEN}$-based plasmid containing the rad9-10 sequence was transformed into a rad $9^{-} \mathrm{CDC} 13^{+}$background and tested for $\mathrm{UV}$ resistance at $23^{\circ}, 30^{\circ}$, and $36^{\circ} \mathrm{C}$ to determine if elevated temperatures affected rad9-10 UV-resistance. By qualitative analysis, the rad9-10-containing strain looked comparable in UV-resistance to a strain containing wild-type $R A D 9$ on a CEN plasmid assaulted with $60 \mathrm{~J} / \mathrm{m}^{2} \mathrm{UV}$ damage and grown overnight, irrespective of the temperature grown at. This was verified by a quantitative analysis of UV-resistance in which, after UV insult, the cells were incubated at $30^{\circ} \mathrm{C}$ overnight to allow time for damage repair (Fig. 2-6a). Cells were also 
assayed at $30^{\circ} \mathrm{C}$ for MMS resistance (Fig. 26b). Consequently, it does not appear that the rad9-10 mutation is temperature-sensitive, indicating that the rad9-10 allele should be defective for blocking DNA degradation at any temperature assayed. Furthermore, this allele should be wild-type in its ability to arrest and repair DNA damage at any temperature tested.

a)

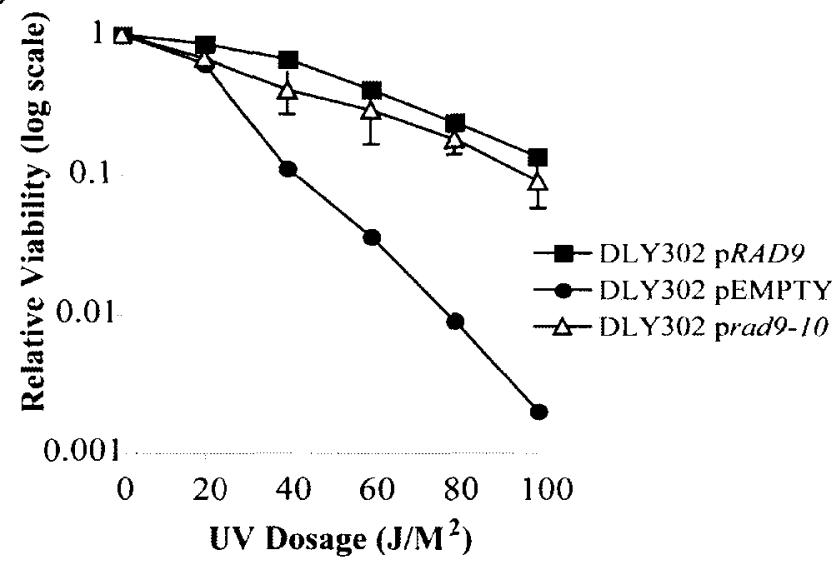

b)

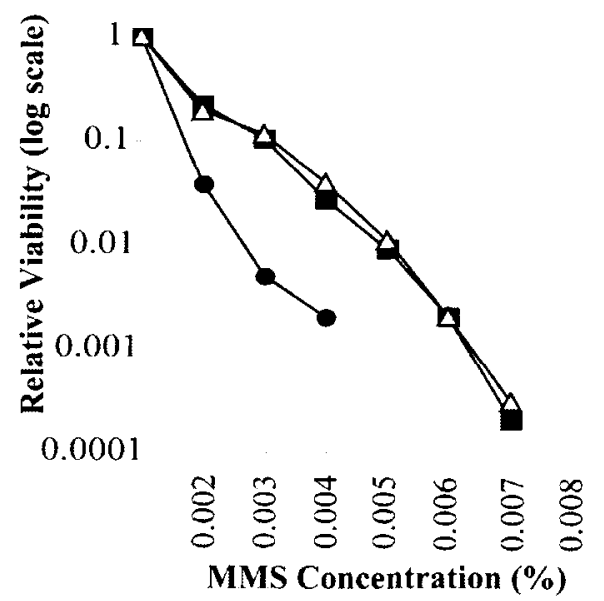

Figure 2-6. UV and MMS sensitivities of rad9-10 assayed at $30^{\circ} \mathrm{C}$. (a) UV sensitivity was determined as described previously, except that cells were incubated overnight at $30^{\circ} \mathrm{C}$ instead of $23^{\circ} \mathrm{C}$. (b) MMS sensitivity was assayed by incubating mid-logarithmic cells in liquid media containing various concentrations of MMS overnight at $30^{\circ} \mathrm{C}$. Cells were then plated the following day to solid media, allowed to grow overnight at $30^{\circ} \mathrm{C}$, and scored the following day for microcolony formation. For each experiment above, the rad9-10 allele was assayed in duplicate. The average of the two duplicates is shown above with error bars showing the standard deviation from the mean. When error bars are not visible, as in panel $(b)$, it is because the error bars do not exceed the symbol used to mark each data point. The DLY302 strain used for these assays is $C D C 13^{+}$.

Based on the prospect for studying the inhibition-of-degradation phenotype that rad9-10 provided, attempts were made to make this a stronger separation-of-function allele. Because the defect in inhibiting degradation was relatively weak, I was suspect that this allele would prove to be robust enough to perform a suppressor screen of inhibition-of-degradation function. Therefore, I employed several different strategies to enhance dysfunction of the rad9-10 insertion mutation in its inhibition-of-degradation phenotype (discussion follows). 
Determining the roles of $M E C l, R A D 53, D U N 1$, and $C H K 1$ in the inhibition-ofdegradation phenotype.

In trying to devise a scheme to make my rad9-10 a stronger separation-offunction allele defective in the inhibition-of-degradation phenotype, I reasoned that the ability of this mutant to carry out a wild-type arrest response might actually be masking the defect in the inhibition-of-degradation phenotype. In other words, rad9-10 may appear to be only hypomorphic for the inhibition-of-degradation phenotype because the ability of this mutant to arrest might be conferring some resistance to the degradation, most likely by allowing time for repair.

To determine if the ability to arrest confers some resistance to the effects of degradation, I mimicked the arrest response in a rad 9 null strain by holding cells in the $\mathrm{G}_{2} / \mathrm{M}$ phase of the cell cycle with the microtubule-inhibiting drug nocodazole after initiation of the inhibition-of-degradation assay. Cells were incubated at the restrictive temperature for a given time, but were then maintained at the permissive temperature for 2 additional hours while still in nocodazole to provide time for DNA repair. Cells were then released from the artificial arrest and plated to solid media, where they were allowed to grow overnight at the permissive temperature and scored for viability the following day (Figure 2-7). I found the results of this experiment to be very striking in that the rad 9 strain subject to the artificial nocodazole arrest closely resembles the phenotype of my rad9-10 allele; both strains are hypomorphic for the inhibition-of-degradation phenotype, lending support to the notion that allowing time for DNA damage repair alleviates the severity of the inability to block DNA degradation. Therefore, I rationalized that knocking out downstream players in the checkpoint arrest response might help to unmask the rad9-10 defect in inhibiting degradation, provided that these proteins were not also involved in inhibiting degradation. 


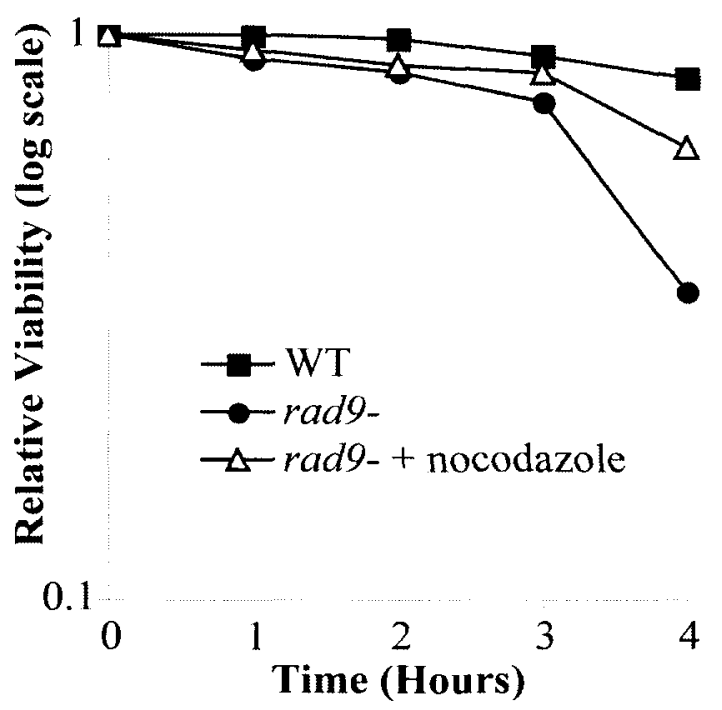

Figure 2-7. Artificial arrest attenuates the inhibition-of-degradation defect. Midlogarithmic cells were synchronized in $G_{1}$ with $\alpha$-factor. Cells were then washed and resuspended in fresh media for release at the restrictive temperature $\left(36^{\circ} \mathrm{C}\right)$. At this time, $10 \mu \mathrm{g} / \mathrm{ml}$ nocodazole was added to one of the $\mathrm{rad} 9^{-}$cultures. At each time point, an aliquot of cells was reserved and incubated at the permissive temperature $\left(23^{\circ} \mathrm{C}\right)$ for 2 additional hours. Cells were then washed with $\mathrm{ddH}_{2} \mathrm{O}$, namely to remove nocodazole from the culture to which it was added, and plated to solid rich media. Cells were incubated overnight at the permissive temperature and scored the following day for microcolony formation to assess viability.

When these experiments were being carried out, the model whereby Rad 9 acts upstream of all the kinases - Mec1, Rad53, Dun, and Chk1 - in the checkpoint cascade was still favored. (We now know that Mec1 localizes to sites of DNA damage with its binding partner, Ddc2, and that Rad9 gets phosphorylated by Mec1 after damage [Emili, 1998; Vialard et al., 1998; Rouse \& Jackson, 2002]. Because Rad9 phosphorylation is required to activate downstream protein kinases, Rad9 likely acts downstream of Mecl and upstream of Rad53 genetically, consistent with biochemical findings [Sanchez et al., 1999; Gilbert et al., 2001; Schwartz et al., 2002].) Thinking that they might be ideal candidates to knock out in order to eliminate the checkpoint arrest response, I sought to determine what role the kinases play in inhibiting degradation. To this end, I performed the quantitative inhibition-of-degradation assay using mecl $\mathrm{smll}$ (the additional $\mathrm{smll}$ 
mutation suppresses loss of the essential $M E C 1$ function in regulating dNTP levels), rad53-1 (this mutation maintains $R A D 53$ 's essential function in regulating dNTP levels), dunl, and chkl strains.

a)

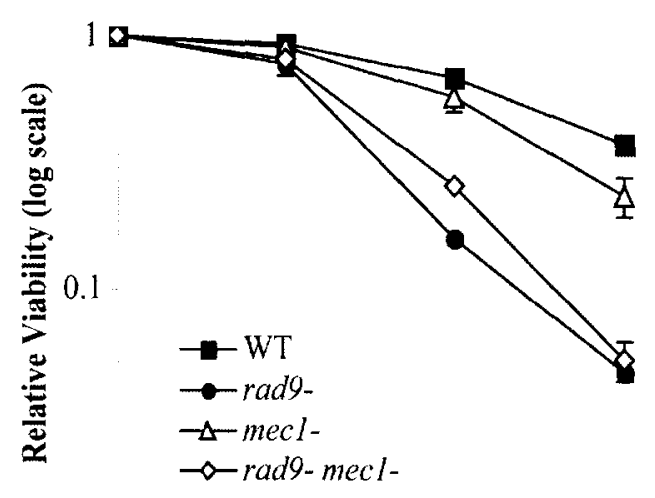

0.01

$\begin{array}{llr}0 & 4 & 4\end{array}$

c)

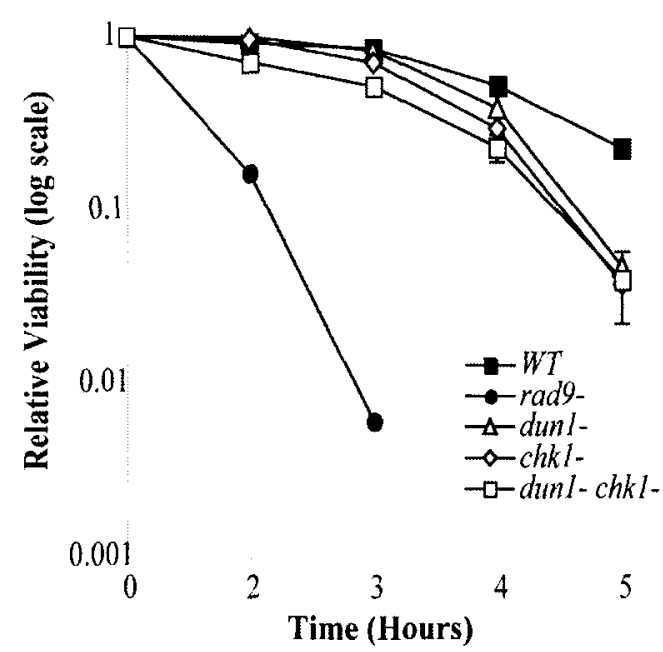

b)

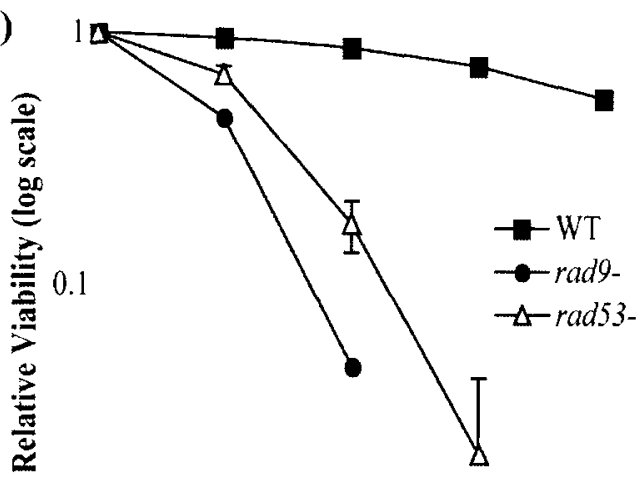

$\begin{array}{cccc}0.01 & 3 & 4 & 5 \\ 0 & & \text { Time (Hours) }\end{array}$

d)

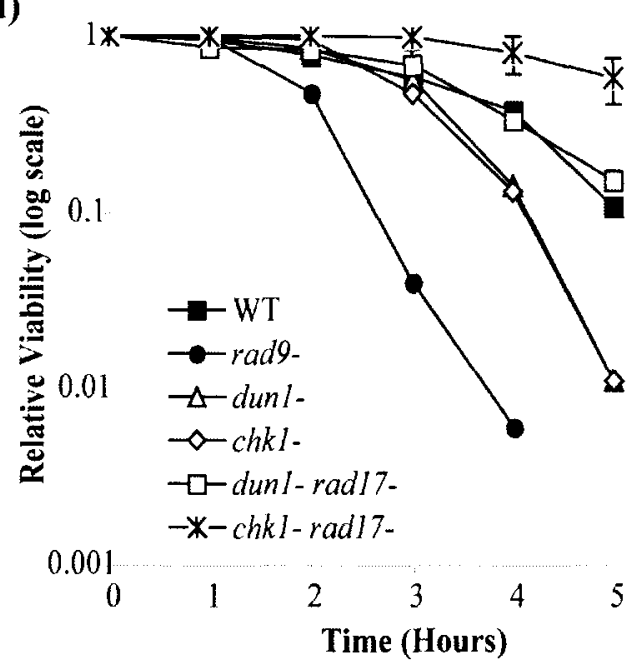

Figure 2-8. Inhibition-of-degradation phenotypes of mecl sml1, rad53-1, dunl, and chkl

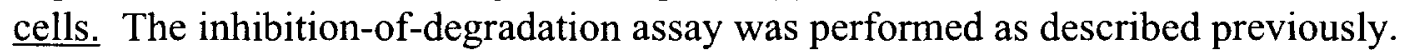
Mutants analyzed include: (a) mecl smll and mecl smll rad9 strains, (b) a rad53 strain, (c) dunl and chkl single mutants and the dunl chkl double mutant, and (d) dunl rad17 and chk $1 \mathrm{rad} 17$ double mutants. All strains tested were performed in duplicate, except for the wild-type and $\mathrm{rad}^{-}$controls. The average of the duplicates is shown above with error bars showing the standard deviation from the mean. Some error bars are smaller than the symbol used to mark the data point, and, thus, do not appear. 
Performing the inhibition-of-degradation assay with a mecl smll strain revealed that $M E C l$ may not be directly involved in inhibiting degradation by $R A D 24$ et al. (Figure 2-8a), as mutating mecl showed only a slight increase in inviability. mecl smll cells are unable to arrest cell cycle progression in the presence of DNA damage, including $c d c 13-1$ damage. Since even $R A D 9^{+}$cells generate some ssDNA degradation mediated by $R A D 24$ et al. and based on the notion that cell cycle arrest helps to restore viability to those cells that have incurred DNA degradation, the slight increase in inviability of $\mathrm{mecl} \mathrm{smll} \mathrm{cells} \mathrm{may} \mathrm{be} \mathrm{due} \mathrm{to} \mathrm{the} \mathrm{loss} \mathrm{of} \mathrm{arrest} \mathrm{that} \mathrm{provides} \mathrm{time} \mathrm{for} \mathrm{DNA}$ damage repair. This is consistent with the fact that a mecl smll rad9 strain shows no increase in inviability over a rad9 strain in the inhibition-of-degradation assay. Both strains cannot prevent DNA degradation due to loss of RAD9 and both strains cannot arrest to provide time repair of degraded DNA, indicating that $M E C I$ does not provide some additional function for preventing degradation.

The same assay performed with a rad53-1 mutant indicates that $R A D 53$ may play a greater role in the function of inhibiting degradation than does $M E C 1$, since the rad53-1 mutant has a greater defect in viability by the inhibition-of-degradation assay but has a known lesser checkpoint arrest defect (Figure 2-8b). If $R A D 9$ acts through $R A D 53$ for the inhibition-of-degradation function, then the greater diminished viability of a rad53-1 strain may be indicative of a more direct defect in inhibiting degradation by RAD24 et al. in addition to the loss of checkpoint arrest that would allow time for damage repair. Because the rad53 mutation does not result in an inhibition-of-degradation defect as severe as that for a rad 9 null, this suggests that $R A D 9$ is mediating some additional function above and beyond that involving $R A D 53$ function. It is also possible that $R A D 9$ acts through $R A D 53$ for inhibiting degradation but the slight increase in viability in a rad53-1 mutant over that of a rad9 mutant is due to the fact that rad53-1 cells can partially arrest, and thus may be proficient for some DNA damage repair, because the CHK1 branch of the checkpoint arrest has not been abrogated. Alternatively, that the 
rad53-1 mutation is not as severe as that in a rad9 null may be due to the fact that rad531 is a hypomorphic allele that perhaps retains some function in preventing DNA degradation.

When dunl and chkl strains were also analyzed for inhibition-of-degradation defects, they demonstrated phenotypes similar to that of mecl smll mutants in the inhibition-of-degradation assay (Figure 2-8c). Each $d u n 1$ and chkl single mutant had a slightly hypomorphic defect in inhibiting degradation. To determine if this defect was dependent on the RAD24 et al. epistasis group that mediates DNA degradation, I tested if deletion of RAD17 (a member of the RAD24 et al. epistasis group) would alleviate the hypomorphic inhibition-of-degradation defect in both $d u n l$ and $\operatorname{chkl}$ strains (Figure 28d). The dun1 rad17 double mutant had a viability comparable to that of wild-type, while the chkl rad17 double mutant was even more viable than wild-type. Because $D U N 1$ and $C H K 1$ act in separate branches of the checkpoint pathway and mutation of each of these genes shows a moderate inhibition-of-degradation defect, I anticipated the dunl chkl double mutant to have an even greater defect in inhibiting degradation. To the contrary, the dunl chkl double mutant showed the same defective level of inhibition-ofdegradation function as each single mutant (Figure 2-8c), suggesting that the two genes are acting in the same pathway to inhibit degradation. This result remains puzzling.

Given these data, it appears that all the checkpoint kinases play some role in inhibiting degradation mediated by RAD24 et al., though not to the same extent. While $M E C 1, D U N 1$, and $C H K 1$ appear to play moderate roles in the inhibition-of-degradation function, $R A D 53$ looks likely to play a greater role as it shows a stronger defect in viability when mutated compared to strains mutated in the other kinase genes.

\section{Creation of $\operatorname{rad} 9$ point mutants not putatively recognized by Rad53.}

Based on the finding that Rad53 might play a role in inhibiting degradation by $\operatorname{Rad} 24$ et al., I tried to create a rad9 mutant that would not associate well with Rad53. 
The rationale was to find a rad9 mutant that could not interact with Rad53 to promote the inhibition of degradation, but that would still maintain UV resistance and the ability to partially arrest by still being proficient for activation of Chkl, located in a branch of the checkpoint cascade separate from that of $\operatorname{Rad} 53$. My hope was to identify a stronger rad9 separation-of function mutant - one that would be close to null in its ability to prevent DNA degradation and hypomorphic in its ability to arrest - to facilitate screening for inhibition-of-degradation suppressor mutations.

The downstream protein kinase Rad53 contains two forkhead-associated (FHA) domains that bind to specific phosphopeptides and are found frequently in DNAassociated proteins (Hofmann and Bucher, 1995; Sun et al., 1998; Durocher et al., 1999; Li et al. 1999; Liao et al., 1999). Initial experiments performed suggested that Rad53's FHA domains bind to phosphorylated Rad9 activated by the DNA damage checkpoint, since mutation of conserved amino acids in the second Rad53 FHA domain disrupted binding to Rad9 and abrogated the activation of Rad53 in vivo (Sun et al, 1998; Durocher et al., 1999). Subsequent work using in vitro peptide libraries identified the binding preferences of Rad53's FHA domains (Liao et al. 1999; Liao et al., 2000; Durocher et al., 2000; Wang et al., 2000). Based on the peptide analysis in two of these reports, it was predicted that Rad53's first FHA domain recognizes and binds to phosphorylated Thr192 in $\operatorname{Rad} 9$ and that Rad53's second FHA domain recognizes and binds to phosphorylated Tyr829 (Liao et al. 1999; Liao et al. 2000). Though tyrosine phosphorylation is not thought to be employed as a common signaling mechanism in yeast, there are dual specificity protein kinases and phospho-Tyr-specific phosphatases in S. cerevisiae (Liao et al., 1999). Hence, the argument was put forth that Rad53's FHA domains might allow for dual specificity in recognizing both phospho-Tyr and phospho-Ser/Thr residues in activated Rad9, though no in vivo experiments supporting this hypothesis were yet performed. 
Based on these preliminary findings, I attempted to disrupt Rad9 and Rad53 association by mutating the putative amino acid residues recognized by Rad53 when phosphorylated. I chose to convert the Thr192 amino acid to an alanine (T192A) and the Tyr829 (Y829F) amino acid to a phenylalanine to minimize changes in the relative size of the amino acid in an effort to maintain protein structure. The T192A and Y829F mutations were created using the QuikChange Site-Directed Mutagenesis Kit that allowed for site-specific mutation in a $R A D 9$ containing plasmid. Basically, two PCR primers containing the desired $R A D 9$ mutations were used to amplify the RAD 9 sequence, creating nicked circular strands containing the desired mutations. The methylated, non-mutated parental $R A D 9+$ vector template was then digested with the restriction enzyme DpnI. Circular, nicked dsDNA was then transformed into competent bacterial cells that repaired the nicks in the mutated plasmids, thereby allowing for amplification of mutagenized rad 9 within the vector.

I was able to successfully create only one of the two desired rad9 point mutants, Y829F. Once I confirmed by sequencing that I had indeed mutagenized the sequence encoding the Y829F point mutation, I subcloned this sequence into an integrating vector and transformed it into a $c d c 13-1 \mathrm{rad}^{-}$strain. I then assayed this mutant by the quantitative arrest, UV resistance, and inhibition-of-degradation assays, comparing against the wild-type and null controls and my strain containing the rad9-10 hypomorphic separation-of-function allele (Figure 2-9). The rad9-Y829F allele shows the exact same phenotype as my rad $9-10$ allele; both are completely proficient for arrest and UV resistance but hypomorphic in their ability to inhibit degradation. Furthermore, the decreased viability of $\mathrm{rad} 9-Y 829 \mathrm{~F}$ in the inhibition-of-degradation assay is $R A D 17$ dependent, consistent with the fact that $R A D 24$ et al., which includes $R A D 17$, is mediating DNA degradation that results in increased cell death when $R A D 9$ is not present or is defective in some way. 
a)

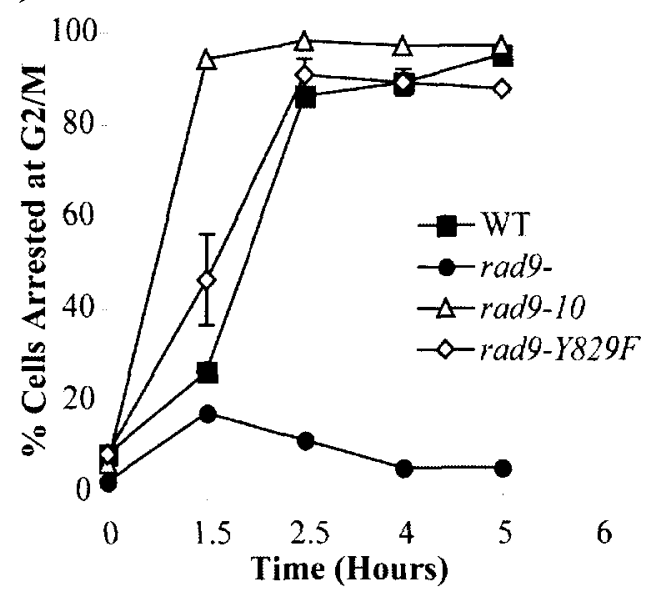

c)

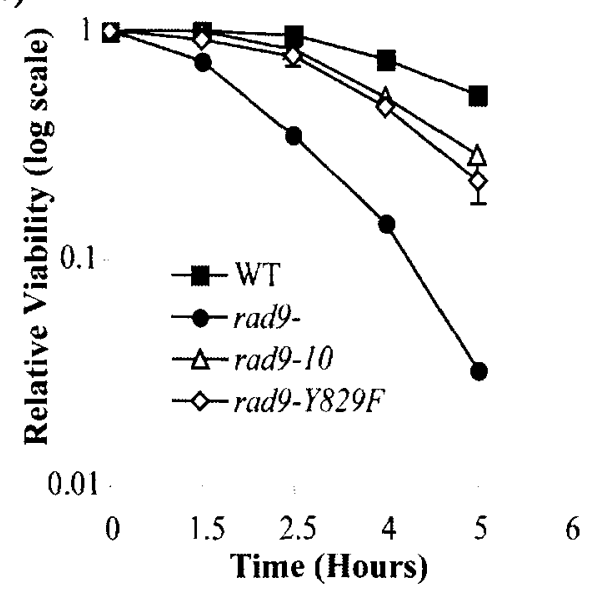

b)

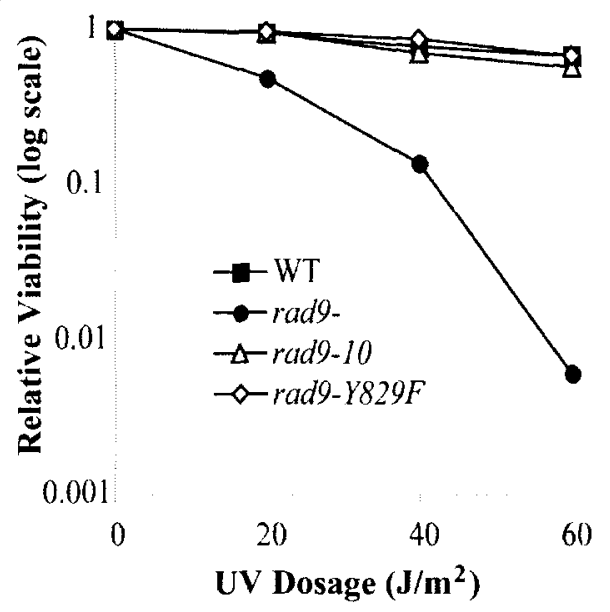

d)

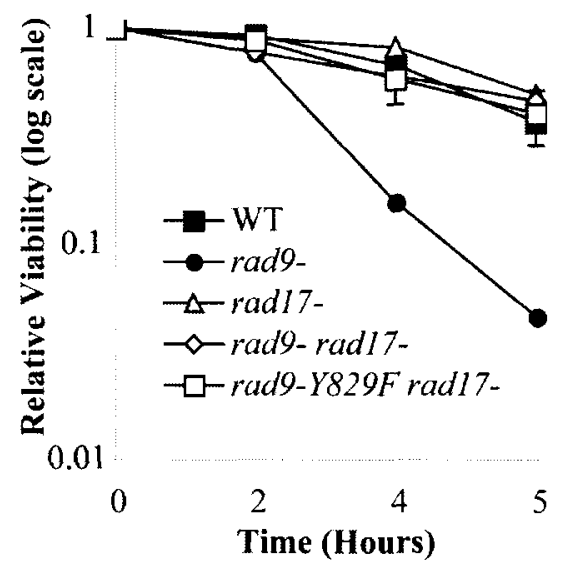

Figure 2-9. Phenotypic analysis of the rad $9-Y 829 F$ allele. Assays were performed as previously described. The rad9-Y829F allele was analyzed for $(a)$ arrest, $(b) \mathrm{UV}$ sensitivity, and $(c)$ inhibition-of-degradation function as compared to the wild-type, rad 9 null, and hypomorphic rad9-10 alleles. (d) The hypomorphic inhibition-of-degradation phenotype of the rad9-Y829F allele is RAD17-dependent. All strains containing rad9$Y 829 F$ were assayed in duplicate. The average of the duplicates is shown above with error bars showing the standard deviation from the mean. 
Direct detection of ssDNA formation in rad9-Y829F.

Because the inhibition-of-degradation assay used in these experiments is an indirect measure of the formation of ssDNA caused by the inability to prevent DNA processing by $R A D 24$ et al., I sought to more directly determine if $\mathrm{rad} 9-\mathrm{Y} 829 \mathrm{~F}$ cells are indeed accruing more ssDNA as compared to wild-type RAD 9 cells. This was accomplished in collaboration with David Lydall's laboratory where they utilized a PCRbased quantitative amplification of ssDNA (QAOS) method to detect the presence and generation of ssDNA at telomere-proximal sites. This method can be used to detect ssDNA levels from $0.2 \%$ to $100 \%$ at single-copy loci in the genome (Maringele \& Lydall, 2002). For this collaboration, I carried out the inhibition-of-degradation assay and purified DNA from cells taken at various time points. The DNA purification scheme is based on procedures from Wu \& Gilbert (1995), Grandin et al. (1997), and the Qiagen Genomic DNA Handbook (see methods for more information). This DNA was then sent to Lydall's laboratory and probed for the presence of ssDNA by QAOS (Booth et al., 2001). The presence of ssDNA was assayed at two locations opposite the AC strand that gets degraded 5' to 3' in $c d c 13-1$ mutants (Figure 2-10a,b) and one control location opposite the TG strand that should not get degraded (Figure 2-10c). 
a)

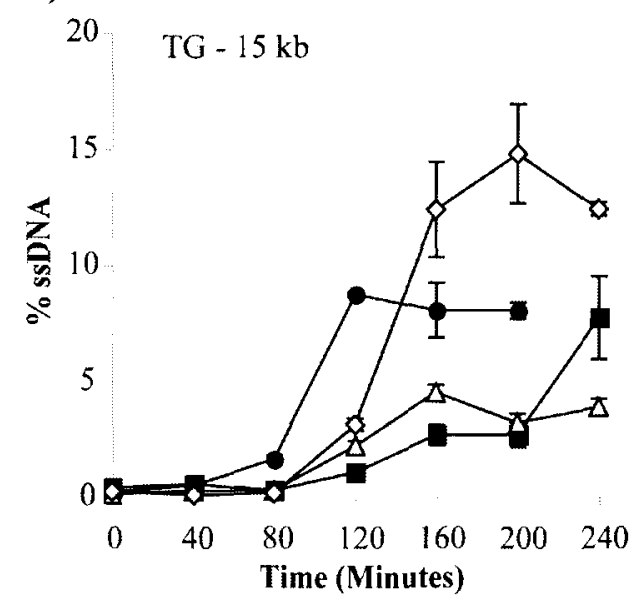

c)

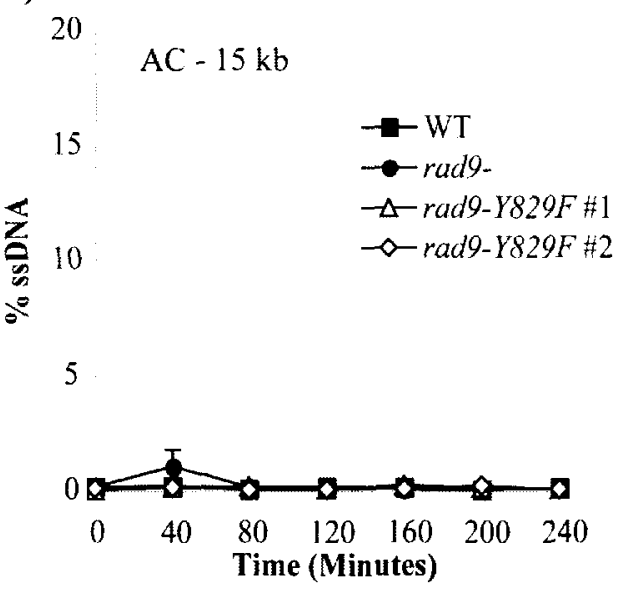

b)

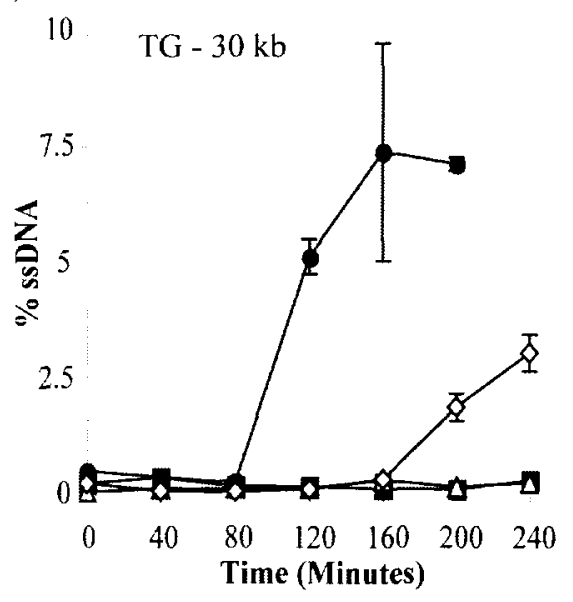

d)

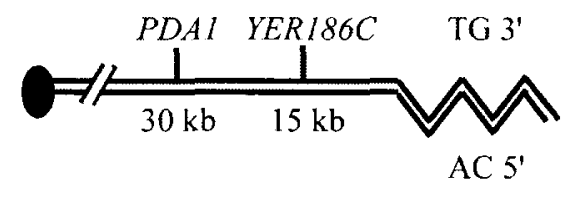

Figure 2-10. Generation of ssDNA in the rad9-Y829F strain. Quantitative amplification of ssDNA generated at $36^{\circ} \mathrm{C}$ by $c d c 13-1$ mutation in the rad $9-Y 829 F$ strain compared to wild-type and rad $^{-}$control strains. The error bars indicate the standard error of the mean derived from three independent measurements of the amount of ssDNA in a sample. (a) Detection of ssDNA on the TG strand $15 \mathrm{~kb}$ from the right end of chromosome $\mathrm{V}$ in budding yeast. (b) Detection of ssDNA on the TG strand $30 \mathrm{~kb}$ from the right end of chromosome V. (c) Detection of ssDNA on the AC strand $15 \mathrm{~kb}$ from the end of chromosome V. (d) A schematic model of the chromosome $\mathrm{V}$ telomere in budding yeast depicting the loci assayed for the presence of ssDNA.

Two independent rad9-Y829F strains were analyzed for the appearance of ssDNA over time upon inactivation of $c d c 13-1$ at $36^{\circ} \mathrm{C}$ (Figure 2-10a-c). A wild-type $R A D 9^{+}$ strain and a null $\mathrm{rad}^{-}$strain were included as positive and negative controls, 
respectively, for the inhibition of degradation. These data are difficult to interpret, since one of the rad9- $Y 829 F$ strains showed a marked increase in ssDNA formation over time, while the other did not. Given the data that indirectly shows that rad9-Y829F cells are partially defective in their ability to inhibit degradation by measuring loss of viability, I hypothesize that the disparate phenotypes seen in the QAOS assay are due to subtleties in growth of different rad $9-Y 829 F$ cultures caused by the hypomorphic nature of the Y829F mutation. While one of the two rad9-Y829F strains in Figure 2-10 does not demonstrate a noticeable increase in ssDNA formation, strain $\# 2$ in this assay does. This strain accumulates TG ssDNA (as the AC strand gets degraded 5' to 3') at the YER186C locus, located $15 \mathrm{~kb}$ from the right end of chromosome $\mathrm{V}$, and also at the more centromereproximal PDAl locus, located $30 \mathrm{~kb}$ from the end of the chromosome. No ssDNA accumulation is seen at the YER186C locus when probed on the AC strand; the TG strand is not subject to degradation and serves as an internal control. Consequently, I regard this data as confirming that rad $9-Y 829 F$ does indeed have a hypomorphic defect in inhibiting degradation as evidenced by the accruement of ssDNA over time, and, at the time, I continued to pursue additional experiments with this allele.

\section{Attempts to create stronger rad9 separation-of-function alleles.}

Based on the previous data that the ability of cells to arrest may attenuate the severity of inviability due to ssDNA formation by allowing time for repair, I created a strain containing the rad9-Y829F mutation in combination with the mecl mutation to abolish checkpoint arrest while presumably not affecting the inhibition-of-degradation pathway. (The smll mutation was also included in this strain to rescue loss of $M E C 1$ 's essential function.) The goal was to create a stronger separation-of-function allele that could be used in a suppressor screen for inhibition-of-degradation function. While loss of mecl did confer a slight increase in the inviability of rad9-Y829F cells, the effect was not as great as I had anticipated (Figure 2-11). 


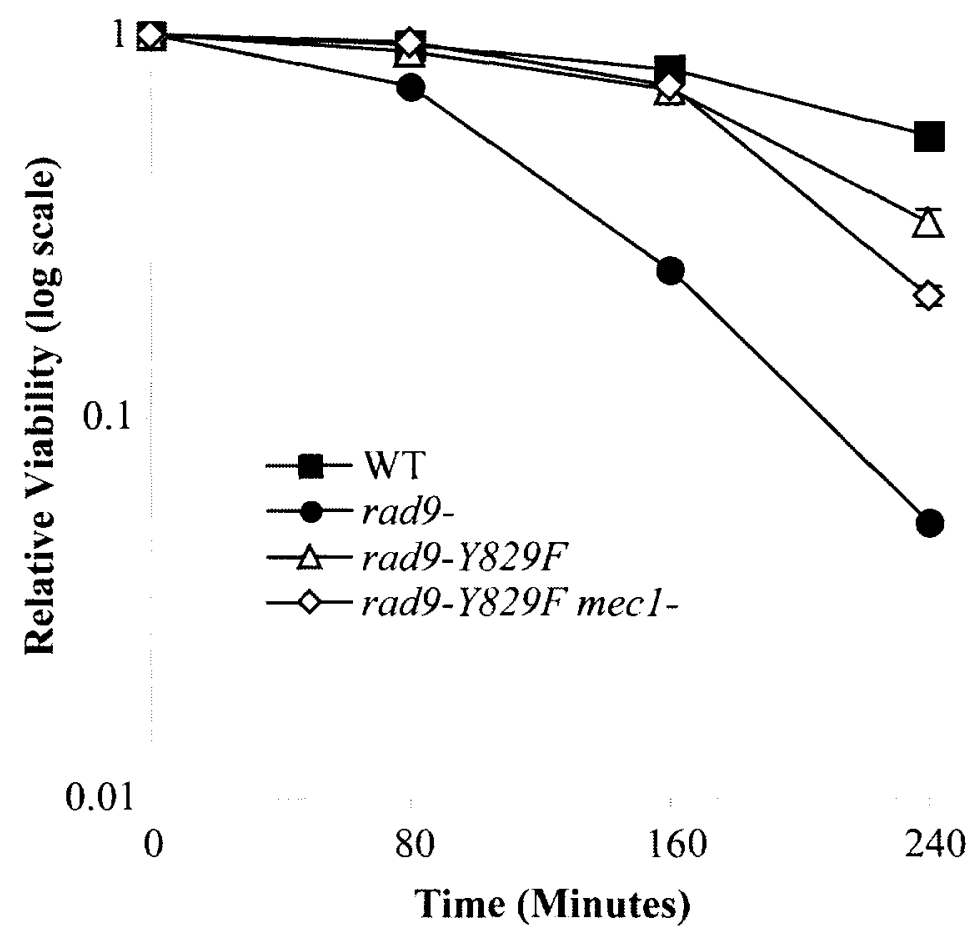

Figure 2-11. A rad9-Y829F mecl smll strain does not show an appreciable increase in defective inhibition-of-degradation function. The inhibition-of-degradation assay was performed as described previously. The assay was performed in duplicate with the rad9$Y 829 \mathrm{~F}$ and rad9-Y829F mecl smll strains, and the average of the duplicates is shown with error bars representing the standard deviation from the mean.

Additional efforts to determine if arrest plays a role in attenuating an inhibition-ofdegradation defect.

In order to better determine the role that arrest might play in attenuating decreased viability due to repair of ssDNA generated by $R A D 24$ et al. degradation, I performed an arrest-release experiment with my two hypomorphic inhibition-of-degradation alleles.

Being that the ability of the $\operatorname{rad} 9-10$ and $\operatorname{rad} 9-Y 829 F$ strains to maintain some viability in the inhibition-of-degradation assay above that of the rad9 null may be due to their ability to arrest and repair ssDNA damage though they are still potentially generating as much 
ssDNA damage as the rad9 null, I hypothesized that these mutated cells may remain arrested for longer than wild-type cells that are not generating as much ssDNA damage since the wild-type cells retain functional $R A D 9$ to help block degradation mediated by $R A D 24$ et al. To test this hypothesis, I incubated cycling cells at the restrictive temperature to inactivate $c d c 13-1$. Cells were left at $36^{\circ} \mathrm{C}$ for 3 hours, at which time the majority of cells had arrested in $\mathrm{G}_{2} / \mathrm{M}$. Cells were then shifted down to the permissive temperature at $23^{\circ} \mathrm{C}$ to restore $C D C 13$ function and to thereby prevent additional ssDNA degradation from the telomere ends. Upon shift-down to $23^{\circ} \mathrm{C}$, cells were fixed at various time points to determine the time at which they resumed cell cycling after being arrested. In theory, this serves to indicate the time at which all ssDNA-generated damage has been repaired and no damage-recognizable DNA remains to activate the checkpoint arrest. Based on this experiment, both rad9 hypomorphs and the wild-type strain all resume cell cycling at the same time, somewhere between 60 and 80 minutes (Figure 212). In addition, there is no appreciable difference between the rate at which they resume cycling and the percentage of cells that resume cycling. 


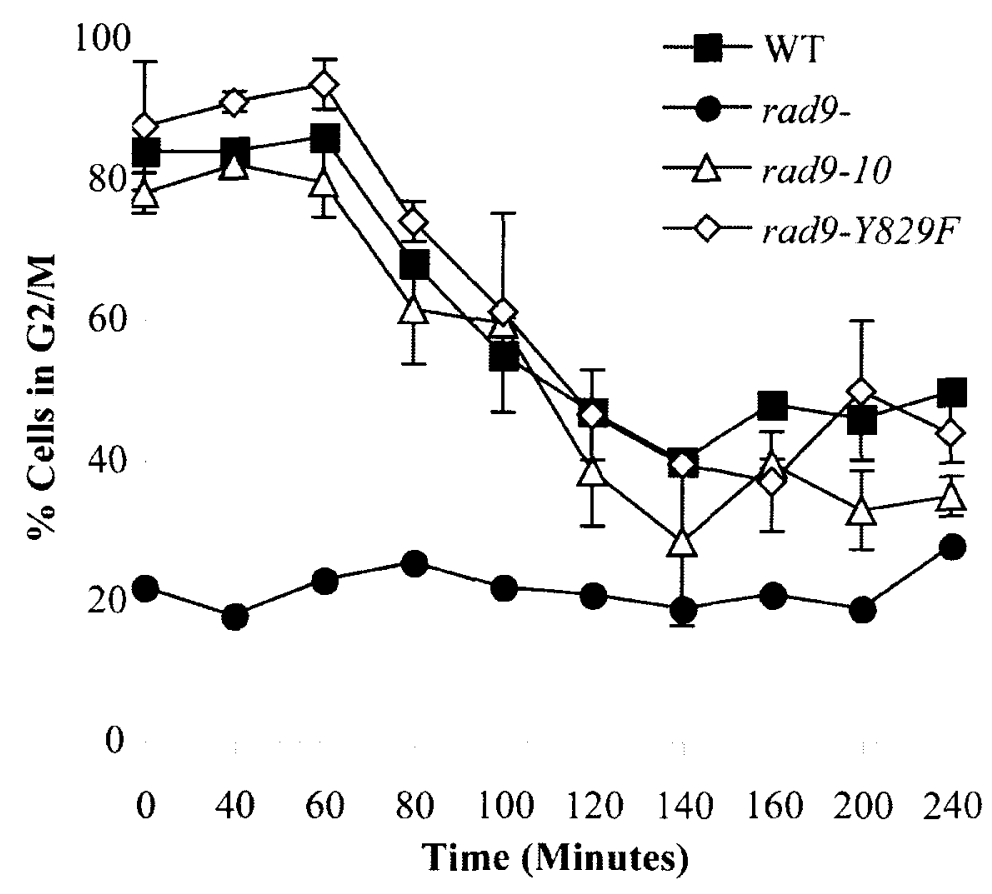

Figure 2-12. Kinetics of cell cycle progression after damage-induced arrest in rad9-10 and rad9-Y829F. Mid-logarithmic cells were shifted to the restrictive temperature $\left(36^{\circ} \mathrm{C}\right)$ for 3 hours to inactivate $c d c 13-1$ and accumulate arrested cells in $G_{2} / M$. Cells were then shifted down to the permissive temperature $\left(23^{\circ} \mathrm{C}\right)$ to restore $C D C 13$ activity and prevent further degradation from chromosome ends. Cells were fixed at various time points to determine the time at which cells resumed cell cycling. The assay was performed in duplicate with the rad $9-10$ and $\mathrm{rad} 9-Y 829 F$ strains. The average of the data is shown with error bars indicating the standard deviation from the mean.

While these results may suggest that the rad9-10 and $\mathrm{rad} 9-Y 829 F$ hypomorphs incur no more ssDNA damage than a wild-type strain, this assumption may not be altogether valid because we do not readily understand the mechanisms of $c d c 13-1$ ssDNA repair. It is formally possible that the rad 9 hypomorphs do indeed sustain more ssDNA damage than a $R A D 9^{+}$strain, as the QAOS PCR results suggest, but that the method of repair of this damage is so efficient in both the wild-type and hypomorphic cells that no obvious difference in the kinetics of release from arrest can be discriminated by this 
assay. Alternatively, the decrease in viability of the rad $9-10$ and rad $9-Y 829 F$ strains in the inhibition-of-degradation assay may not be due to more extensive generation of ssDNA damage but, rather, may be due to defects in repair of this damage. (This notion is not likely given the ssDNA PCR data shown in Figure 2-10.) Again, $R A D 9^{+}$cells generate some ssDNA damage when $c d c 13-1$ is inactivated. If $R A D 9$ cells act to inhibit DNA degradation by efficient repair of ssDNA formation, then the hypomorphic rad9-10 and $\mathrm{rad} 9-Y 829 \mathrm{~F}$ alleles may be hampered in their ability to effectively repair such damage. The kinetics of such error-prone repair may be similar to that of faithful repair in wild-type cells; it is simply the fidelity of repair that is abrogated and, hence, causes death due to mutation.

Given the doubt that the arrest-release experiment cast on the ability of cell cycle arrest to restore some viability to cells sustaining ssDNA damage from telomere ends, no further experiments were pursued in attempting to eliminate cell cycle arrest in my hypomorphic rad9-10 and rad9-Y829F strains to create a stronger separation-of-function mutant. Instead, because the nature of the mutations that results in the hypomorphic phenotype of these alleles is unknown, I speculated that combining the two mutations in the same gene might create a more defective inhibition-of-degradation allele. Therefore, I cloned the rad9-10, Y829F double mutant gene and integrated into a $c d c 13-1$ rad 9 strain. I then tested the ability of the strain containing this doubly-mutated gene to arrest, inhibit degradation, and maintain viability after UV irradiation treatment. Combining the two mutations had no effect on UV resistance and the ability of cells to arrest; the 
rad9-10, $Y 829 F$ strain still remained wild-type for these functions. Moreover, combining the rad9-10 and $Y 829 F$ mutations created no additional decrease in viability in the inhibition-of-degradation assay as compared to each single mutant strain (Figure 2-13). This suggests that each individual mutation responsible for the hypomorphic inhibitionof-degradation of phenotype likely causes the same defect within this pathway, as combining the two mutations creates no additional defect.

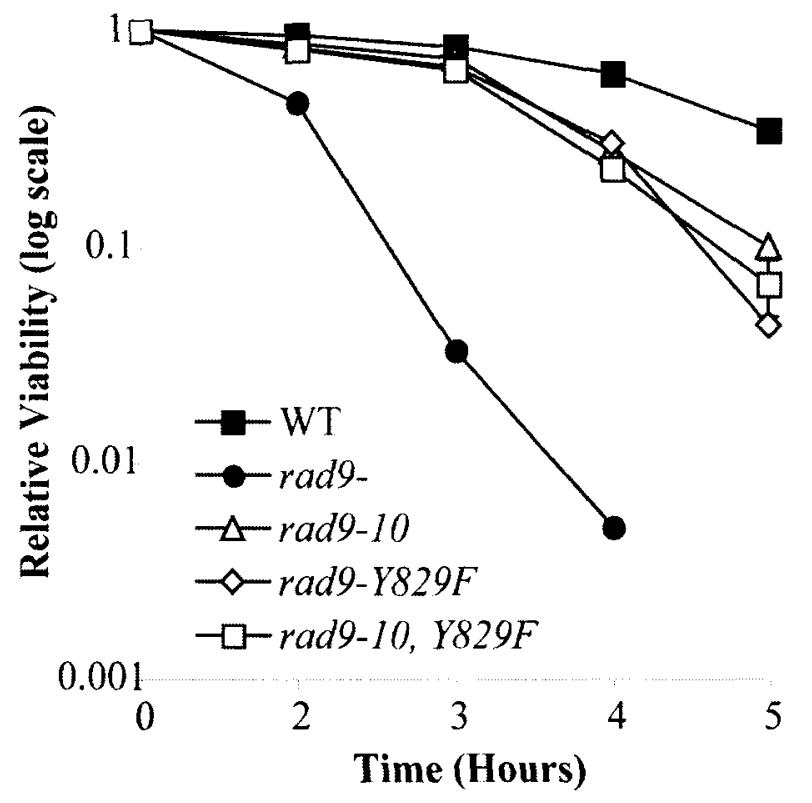

Figure 2-13. The doubly-mutated rad9-10,Y829F allele has the same inhibition-ofdegradation defect as each single mutant alone. The inhibition-of-degradation assay was performed as described previously. The rad $9-10, Y 829 \mathrm{~F}$ strain was assayed in duplicate. The average of the duplicates is shown above, with error bars representing the standard deviation from the mean.

Determining the role of TOFI in the inhibition-of-degradation phenotype. During the course of these experiments, a paper was published showing that a rad 9 tofl double mutant has a synergistic sensitivity to MMS, UV, and HU damage (Foss, 2001). TOF1 (TOpoisomerase 1 -associated $F$ actor 1 ) encodes a protein that interacts with the 
topoisomerase Top1 by two-hybrid and in vitro assays (Park \& Sternglanz, 1999). The data in the 2001 paper implicate $R A D 9$ and $T O F 1$ as acting redundantly during S-phase to signal checkpoint arrest upon recognition of DNA damage and/or stalled replication forks. Of interest, $c d c 13-1$ damage is only generated in those cells that proceed through S-phase, possibly because replication of chromosome ends would displace functional Cdc13 from telomeres and then leave them unprotected upon Cdc13 inactivation (Garvik et al., 1995). Consequently, I reasoned that the addition of the tofl mutation in my hypomorphic inhibition-of-degradation strains may synergistically increase the aberrant phenotype to such a low level of inviability that would be useful for a suppressor screen.

To test this notion, I first checked to determine if a rad9 tof 1 double mutant is more sensitive to DNA degradation by performing the inhibition-of-degradation assay. These $c d c 13-1$ tofl and $c d c 13-1$ rad9 tof 1 strains were created by PCR amplification of the tof $1: \because K A N$ knockout sequence from the EUROSCARF (European Saccharomyces cerevisiae Archive for Functional Analysis) knockout collection (kindly provided by Roy Parker), followed by integration of this PCR fragment into $c d c 13-1$ and $c d c 13-1$ rad9 strains. After selection, tofl knockouts were confirmed by additional PCR analysis.

The results for the inhibition-of-degradation assay show that the tofl mutation confers no marked additional increase in sensitivity to DNA degradation in rad9 cells, as was seen when rad9 tofl double mutants were treated with MMS or HU damage (Figure 2-14; Foss, 2001). This suggests that TOF1 plays no role in inhibiting DNA degradation. 
Hence, I did not pursue the proposed experiments that would have combined my hypomorphic rad9-10 and rad9-Y829F alleles with the tof1 mutation.

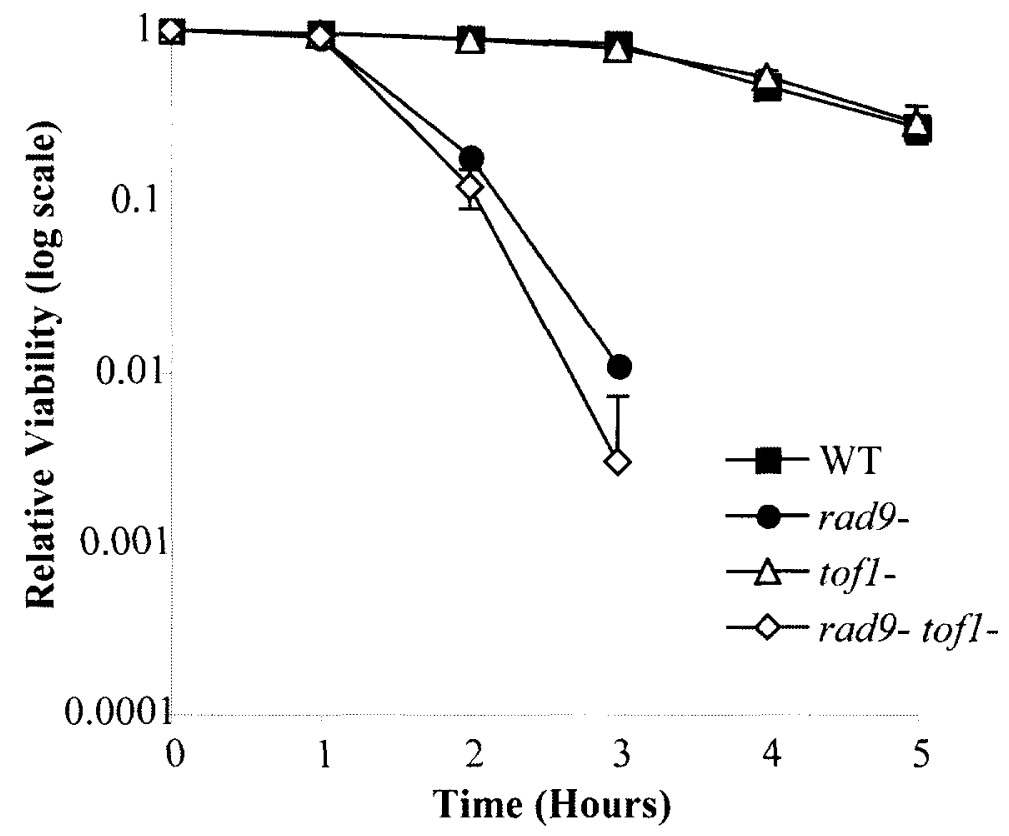

Figure 2-14. tof1 mutation does not create a synergistic defect in rad 9 inhibition-ofdegradation dysfunction. The inhibition-of-degradation assay was performed as described previously. Strains containing the toflmutation were performed in duplicate. The average of the duplicates is shown above along with error bars representing the standard deviation from the mean.

The role of $A S F 1$ in inhibiting DNA degradation.

In 2001 , two papers were published that I found very intriguing in potentially helping to explain the underlying mechanisms involved in the inhibition-of-degradation function. These papers directly linked the DNA damage checkpoint protein Rad53 to the chromatin assembly factor Asfl (Emili et al, 2001; Hu et al, 2001). Asfl belongs to a conserved multi-subunit replication-dependent chromatin assembly complex that acts during normal S-phase and during repair of double-stranded DNA breaks. Asfl binds to 
acetylated histone subunits $\mathrm{H} 3$ and $\mathrm{H} 4$ in vivo and mediates their deposition onto newly replicated DNA in vitro, necessary for formation of nucleosomes on DNA (Tyler et al., 1999). The first paper identified Asfl as binding to Rad53 in a screen for Rad53interacting proteins using protein affinity purification and tandem mass spectrometry (Emili et al., 2001). The second paper identified Asf1 as a suppressor of the temperaturesensitive phenotype of rad53 $\mathrm{mrcl}$ double mutants and the HU sensitivity of rad53 mutants (Hu et al., 2001). Both papers provided evidence that Asfl preferentially binds to the hypophosphorylated form of Rad53. When bound to Rad53, Asf1 is blocked from associating with the acetylated $\mathrm{H} 3$ and $\mathrm{H} 4$ histones. Upon Rad53 hyperphosphorylation, however, which is Mecl-dependent and occurs when the checkpoint cascade is activated by the presence of DNA damage, Asfl dissociates from Rad53 and can then interact with acetylated $\mathrm{H} 3$ and $\mathrm{H} 4$ to mediate histone deposition on DNA.

While the authors speculated that Asf1 plays a role in repair of damaged DNA by depositing nucleosomes on newly replicated DNA, I conjectured about another related role for Asf1 in inhibiting DNA degradation. We know Rad53 activation to be Rad9dependent in the $G_{2} / M$-phase of the cell cycle. We also know that $\operatorname{Rad} 9$ and possibly Rad53, as shown earlier in this work, play a role in inhibiting degradation mediated by $\operatorname{Rad} 24$ et al. Given this, $\operatorname{Rad} 9$ activation of $\operatorname{Rad} 53$ should induce Asf1 disassociation. Asfl, then, should be free to deposit histones onto DNA. Histone deposition leads to nucleosome formation that then leads to a condensation of chromatin when histones are deacetylated. Hence, I deduced that if resected ssDNA gets filled in by a polymerase, 
then Asf1 may help to create a physical block to DNA degradation by contributing to the condensation of chromatin structure to inhibit additional exonuclease activity. If such a role for Asfl were true, then a strain mutated for asfl would be predicted to have an inhibition-of-degradation defect in the presence of $c d c 13-1$ damage and this defect would be RAD24 et al. dependent. Consequently, I set out to determine the phenotype of a cdc13-1 asf1 mutant.

I initially requested the $c d c 13-1$ asfl strain and control strains (wild-type, $c d c 13-1$, and asf1) from Andrew Emili. (It should be noted that these mutations are in the $S$. cerevisiae A364a strain background.) Performing the inhibition-of-degradation assay with these strains revealed that a $c d c 13-1$ asfl mutant does indeed have a defect in viability that is assumed to be due to its inability to prevent degradation (Figure 2-15a). To verify this, I additionally mutated rad 17 in the $c d c 13-1$ asf 1 strain. As hoped, the addition of the rad 17 mutation to the $c d c 13-1$ asfl strain restored viability, indicating that the growth defect in $c d c 13-1$ asfl cells is due to an inhibition-of-degradation defect that can be overcome by eliminating those genes responsible for promoting degradation. 
a)

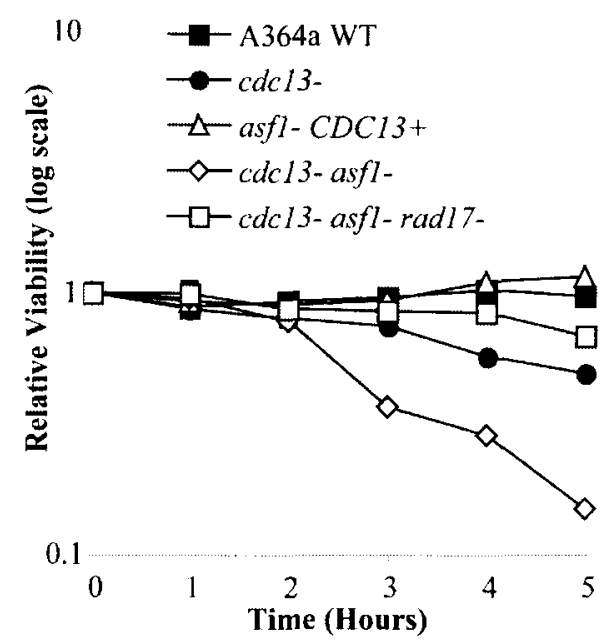

b)

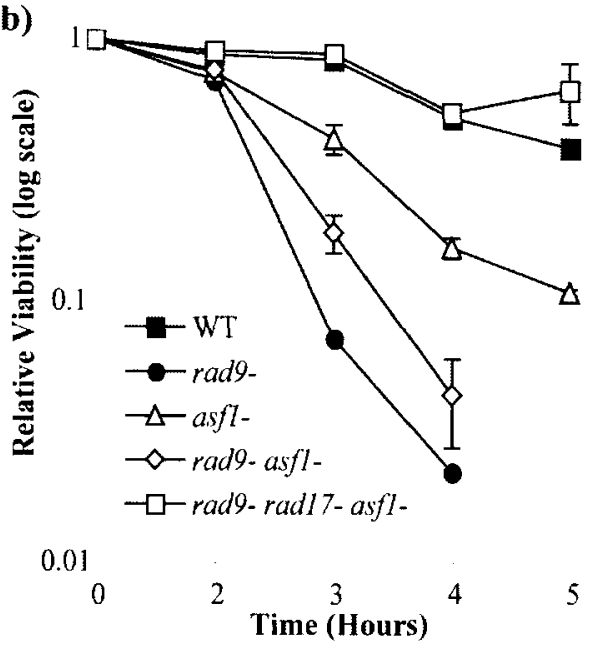

Figure 2-15. A cdc13-1 asf1 mutant shows an inhibition-of-degradation defect. The inhibition-of-degradation assay was performed as described previously. Panel $(a)$ shows the assay performed in the A364a yeast strain background, while panel $(b)$ shows the results of the assay performed in the $\mathrm{W} 303 \mathrm{cdc} 13-1$ yeast background used in all other experiments. In panel $(b)$, all strains containing asfl were performed in duplicate. The average of the duplicates is shown with error bars representing the standard deviation from the mean.

I next analyzed the asf1 mutations in our W303 $c d c 13-1$ yeast strain background used in all other experiments. The asfl mutation was created by PCR amplification of the asf $1:: K A N$ cassette from the EUROSCARF strain (provided by Roy Parker). After transformation of this PCR fragment into yeast, all asfl::KAN deletions were confirmed by additional PCR analysis. These strains were then assayed for the inhibition-ofdegradation phenotype. As in the A364a strain background, $c d c 13-1$ asfl mutants have an inhibition-of-degradation defect in the W303 strain background (Figure 2-15b). This defect is not as great as that of a $c d c 13-1$ rad9 null, and addition of the rad9 mutation to a cdc13-1 asfl strain causes no greater inhibition dysfunction than a $c d c 13-1$ rad9 null alone. Strikingly, the addition of the rad17 mutation to the $c d c 13-1$ asfl rad9 triple 
mutant completely relieves the inviability of this strain. Given these data, I find it very convincing that $A S F 1$ plays a role in inhibiting DNA degradation mediated by $R A D 24$ et al., and there are several experiments that should be performed to pursue this possibility.

In collaboration with David Lydall, we are again using his quantitative PCR method to directly determine if asfl cells show an increased accumulation in ssDNA after cdc13-1 damage compared to wild-type cells. The results of this experiment should definitively assess whether or not $A S F 1$ does indeed play a role in inhibiting degradation.

Experiments that would confirm and further test Asfl's role in the inhibition-ofdegradation function.

Additional experiments that would solidify a role for $A S F 1$ in the inhibition-ofdegradation function include genetic and molecular tests. Genetic experiments should be performed to confirm that both $A S F 1$ and $R A D 53$ act downstream of $R A D 9$ for this function if indeed phosphorylation of Rad9 leads to Rad53 activation that causes Asfl release for histone deposition on DNA. By this reasoning, using a constitutively active $R A D 53$ allele in a rad 9 null should maintain cell viability in the inhibition-of-degradation assay because Asf1 would always be free for histone deposition on DNA. Alternatively, overexpressing $A S F 1$ in a rad9 rad53 background may restore resistance to DNA degradation; partial restoration would even be informative as it would suggest that Asf1 interactors may be rate-limiting for function. Molecular tests include looking to see if Asfl localizes to sites of $c d c 13-1$ damage, either using chromatin immunoprecipitation (CHIP) analysis or GFP localization of Asfl at damage sites. 
I did GFP-tag Asf1 in order to perform the localization experiments described above. In addition, I GFP-tagged Ddc1 as a positive control, as it has been shown that this protein also localizes to sites of DNA damage (Kondo et al., 2001; Melo et al., 2001). In my very preliminary analyses, I saw that both Ddc1-GFP and Asfl-GFP were diffusely present in the nucleus in non-damaged cells. In contrast, upon $c d c 13-1$ inactivation, I saw foci form in both Ddc1-GFP and Asfl-GFP cells (Figure 2-16). I have not yet determined if the Asf1 focus formation is Rad9-dependent, as it is with Ddc1, and I have also not quantified the number of cells that form foci or the number of foci per cell. Ddcl can be seen in 2-3 foci per each cell subject to $c d c 13-1$ damage (Melo et al., 2001). Consequently, I would expect to see similar amounts of foci formation with the Asfl-GFP if it is indeed localizing to sites of DNA damage to help block additional DNA degradation. 
a)

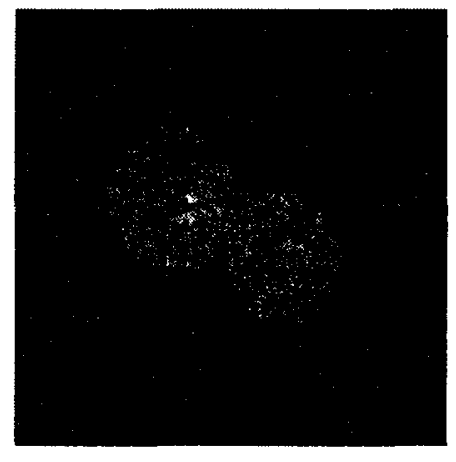

b)

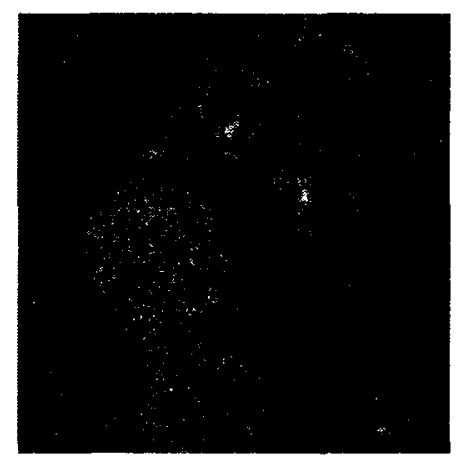

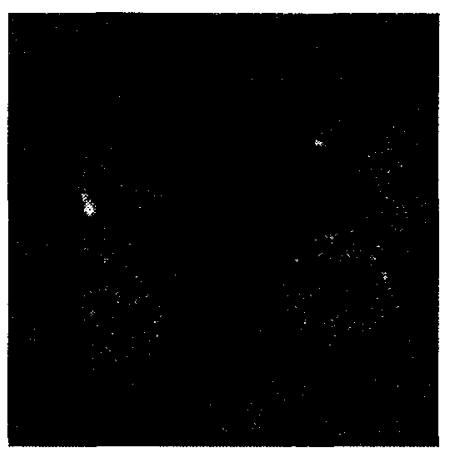

Figure 2-16. Asf1-GFP foci formation after $c d c 13-1$ damage induction. Mid-logarithmic cells were shifted to the restrictive temperature for 3 hours to induce $c d c 13-1$ damage and arrest. Cells were then analyzed by fluorescent microscopy to determine the presence of GFP-labeled foci. (a) Ddcl-GFP focus formation in the presence of $c d c 13-1$ damage. (b) Two images of Asfl-GFP focus formation in the presence of $c d c 13-1$ damage. Note that not all Asfl-GFP cells contain foci.

In summary, I find the data persuasive that Asfl and Rad53 play a significant role in inhibiting DNA degradation mediated by the Rad24 et al. proteins. as 1 clearly has an inhibition-of-degradation defect that is $R A D 17$-dependent. It also appears that it might form foci in the presence of $c d c 13-1$ damage, suggesting that these foci might co-localize with sites of damage. Given that Asfl association with Rad53 is disrupted upon Rad53 activation to allow for Asfl interaction with acetylated histones and deposition of these histones onto DNA, I encourage future study of the role of Asfl in inhibiting DNA degradation. 


\section{CONCLUSION}

In summary, I was successful in identifying rad9 separation-of-function mutants that were proficient for the DNA damage arrest and repair responses but that were hindered in their ability to prevent DNA degradation mediated by RAD24 et al. These mutant alleles were identified by two different methods, rad $9-10$ being discovered in a pentapeptide insertional mutagenesis screen and rad $9-Y 829 F$ being created by point mutation after gleaning information from the literature. Unfortunately, these two mutants were not robust enough in their inhibition-of-degradation defects to pursue additional suppressor analysis of the inhibition-of-degradation function. Several attempts were taken to create greater inhibition-of-degradation dysfunction in these mutants by combining them with other mutations known to eliminate checkpoint arrest, in the hope that doing away with the checkpoint arrest would not allow time for repair of degraded DNA and enhance its persistence. I also combined the two hypomorphic mutations within the same allele - rad9-10,Y829F - to try to enhance the defect in inhibiting DNA degradation. Unfortunately, neither effort was fruitful.

Perhaps my inability to create a stronger $\operatorname{rad} 9$ separation-of-function allele defective in inhibiting DNA degradation should be regarded as informative in thinking about the mechanism of degradation inhibition. If the inhibition-of-degradation function requires similar checkpoint proteins to those that function in the DNA damage arrest and repair pathways, then it may not be possible to identify a stronger rad9 separation-of- 
function mutant than the ones I already have without also affecting the arrest and repair responses. In other words, the biology of the rad9-10 and rad9-Y829F alleles may indicate that it is not possible to create a stronger separation-of-function allele because the components involved are the same as those needed for checkpoint arrest and repair. As discussed previously, the hypomorphic alleles that I identified may be hypomorphic because the threshold levels of protein activity may be different for the inhibition-ofdegradation and the arrest functions, though the protein components involved are similar. The damage arrest signal is amplified by Rad53 and Chk 1 protein kinase activities after being activated by Rad9. The inhibition-of-degradation function, however, may not be subject to the same levels of amplification, and, consequently, a greater level of Rad9 protein activity may be required for full function of this response. For example, if Asfl dissociation from Rad53 is required for the deposition of histones on DNA to condense chromatin structure in order to prevent DNA degradation, then one would predict that at least one Rad53 molecule needs to be activated at each site of damage for chromatin restructuring upon Asfl release. In contrast, regardless of the amount of damage, one activated Rad53 molecule has the potential to phosphorylate numerous downstream substrates to arrest the cell cycle as long as it remains activated. Therefore, the separation-of-function alleles that I have identified may be abrogated in function just enough to affect the inhibition-of-degradation phenotype while leaving the arrest and repair functions unaffected. 
If indeed it is not possible to separate the inhibition-of-degradation and arrest functions any more than I have already done, then I suggest a more directed approach in studying the block to degradation. By the data I have collected thus far, I am compelled to believe that both Rad53 and Asf1 play a critical role in inhibiting DNA degradation. Both mutants demonstrate a significant inhibition-of-degradation defect that is RAD17dependent, and both proteins are known to act downstream of $R A D 9$ after its activation. It has yet to be determined if $R A D 53$ and $A S F 1$ are acting to promote the block to DNA degradation, but a collaborative effort with David Lydall's laboratory is underway to address this possibility. 


\section{CHAPTER 3. THE BRCT REPEATS OF THE RAD9 CHECKPOINT PROTEIN ARE REQUIRED PREDOMINANTLY FOR CONCENTRATING RAD9}

\section{Statement by author}

The work presented in this chapter is being prepared in a manuscript for peer review and publication, contingent upon the completion of a few additional experiments. I performed all the work presented herein, with help from Ted Weinert in growing the Gal overexpression strains in Figure 2-2a that I subsequently prepared for protein purification and Western analysis. Additionally, Weinert scored these cells for their ability to arrest, shown in Figure 2-2c. Future co-immunoprecipitation and protein localization analysis will be completed by Weinert.

\section{SUMMARY}

The Saccharomyces cerevisiae Rad9 checkpoint protein contains two BRCT domains, a domain with several possible functions present in many proteins with roles in DNA metabolism. Using in vivo methods, we investigated how Rad9's BRCT domains function in activation of important protein kinases $(\operatorname{Rad} 53$, Chk1) to cause cell cycle arrest and DNA repair. We found that removal of Rad9's BRCT domains (rad9 $\triangle B R C T$ allele) resulted in complete inactivation of $\operatorname{Rad} 9$ to either interact with or activate $\operatorname{Rad} 53$ and Chk1, thereby abrogating cell cycle arrest and repair of UV-induced DNA damage. However, we find that Rad9's BRCT repeats are not essential per se because overexpression of the rad9 $\triangle \mathrm{BRCT}$ protein almost completely restored all cellular 
functions to rad $9 \triangle B R C T$. This suggests that the BRCT repeats may normally act simply to increase Rad9's local concentration. We also found that complete function was restored to the $\operatorname{rad} 9 \triangle B R C T$ allele by fusion to the dimer-competent GST protein. The $\operatorname{rad} 9 \triangle \mathrm{BRCT}$-GST fusion, expressed at low levels when compared to the wild-type Rad9 protein, was completely functional for interaction with Rad53 and Chk1 protein kinases, $\mathrm{S}$ - and $\mathrm{G}_{2} / \mathrm{M}$ - phase cell cycle arrest, and conferred MMS and UV resistance. Finally and surprisingly, genetic analysis of point mutations within Rad9's BRCT domains, present in mutated alleles of human $B R C A 1$, suggests that the BRCT repeats may also negatively regulate $\operatorname{Rad} 9$ function or may influence protein localization. In sum, we conclude that Rad9's BRCT domains are needed predominantly for Rad9 concentration, conferred by homodimerization and protein stabilization. We predict that homodimerization by the BRCTs increases Rad9 local concentration to enable Rad9-dependent reactions. Furthermore, the BRCT repeats may have a second role in negative regulation and/or protein localization. 


\section{INTRODUCTION}

$\operatorname{Rad} 9$ is one of several checkpoint proteins operating in the budding yeast $S$. cerevisiae. The molecular roles of Rad9 are now coming to light. In a current model, Rad9 interacts with proteins that directly recognize DNA damage to form a complex that functions to propagate an arrest response by activating downstream protein kinases Rad53 and Chk1 (Navas et al., 1996; Sanchez et al., 1999). Directly binding to DNA damage are the Mec1-Ddc2 and Rad24-Rad17-Mec3-Ddc1 groups of checkpoint proteins (Melo et al., 2001; Kondo et al., 2001). Presence of these proteins at damage leads to Mec1-dependent hyperphosphorylation of $\operatorname{Rad} 9$ - a protein modification required for $\operatorname{Rad} 9$ interaction with the downstream protein kinase Rad53 (Schwartz et al., 2002; Sun et al., 1998; Emili, 1998; Vialard et al., 1998). Other have shown that Rad9 also dimerizes under damaging conditions, and the combined effect of $\operatorname{Rad} 9$ phosphorylation and its dimerization brings two Rad9-associated Rad53 molecules in close enough proximity to induce their autophorphorylation (Soulier \& Lownes, 1999; Gilbert et al., 2001). Additionally, Rad9 activates the Chk1 protein kinase, though the molecular details leading to Chk1 activation are unclear (Sanchez et al., 1999).

Key molecular questions in Rad9 biology include: 1) how does Rad9 get to DNA damage, 2) how does Mec1 recognize Rad9, 3) what role does the Rad24-Rad17-Mec2Ddc1 complex play in Rad9 phosphorylation, 4) does Rad9 phosphorylation induce dimerization, and, 5) what Rad9 modifications are necessary for Chk1 activation? 
Answers to these questions might be found in understanding the role of Rad9's only

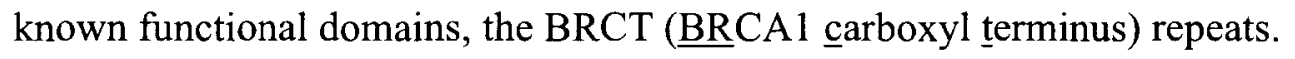

First identified in the C-terminus of the human breast cancer susceptibility protein BRCA1, the BRCT domains occur frequently in proteins involved in DNA damage response (Koonin et al., 1996; Bork et al., 1997; Callebaut \& Mornon, 1997). More than 50 BRCT-containing proteins have now been identified in essentially all organisms (Huyton et al., 2000). Examples include the fission yeast replication checkpoint protein $\mathrm{Rad} 4$, the mammalian DNA repair protein $\mathrm{XRCC}$, the budding yeast DNA polymerase subunit Dpb11, and the bacterial DNA ligase LigA. Though the exact molecular functions of these proteins are diverse, they all play some role in responding to DNA damage. Moreover, all BRCT-containing proteins possess other large domains with distinct activities, possibly suggesting that the BRCTs play a role in signal transduction, linking together proteins that process or control DNA structure (Bork et al., 1997). Since all BRCT-containing proteins are involved in some aspect of DNA metabolism, some DNA function is implied.

The roles of the BRCT domains in any protein have yet to be fully characterized. The majority of the literature suggests that the BRCT domains mediate protein-protein interactions, including homotypic dimerization of proteins via their BRCT domains and other heterotypic BRCT-nonBRCT interactions, while other studies implicate the BRCT domains in DNA binding. For example, the XRCC1 protein, which functions in the base 
excision repair pathway as a scaffolding protein, contains two BRCT domains that each interact with the BRCT domains within the proteins DNA ligase III and poly ADP ribose polymerase (Caldecott et al., 1995; Nash et al., 1997; Taylor et al., 1998; Masson et al., 1998). BRCT dimerizaton is supported by XRCC1 BRCT crystal structure analysis that reveals a hydrophobic, autonomously folded domain with a globular structure consisting of a four-stranded parallel $\beta$-sheet core surrounded by three $\alpha$-helices (Zhang et al., 1998). Moreover, two crystal structures of an XRCC1 BRCT domain suggest two different packing arrangements - it has been suggested one for inter-BRCT associations between two proteins and one for intra-BRCT associations between domains within the same protein (Huyton et al., 2000). Other work indicates that the BRCT domains in $\mathrm{XRCC} 1$ and in other proteins function to bind DNA strand breaks and DNA termini. $\mathrm{XRCC1}$, the topoisomerase binding protein (TopBP1), and BRCA1 each bind to DNA via their BRCT domains (Yamane et al., 2000 (BRCA1 \& XRCC1); Yamane \& Tsuruo, 1999). Of note, BRCT-mediated binding occurred at both single- and double-stranded DNA ends and nicked circular DNA, but not to circular double-stranded DNA, and binding was not dependent on DNA sequence (Yamane \& Tsuruo, 1999; Yamane et al., 2000 (BRCA1 \& XRCC1)). Furthermore, protein binding conferred exonucleaseresistance to the ends of the DNA fragments bound (Yamane \& Tsuruo, 1999; Yamane et al., 2000 (BRCA1 \& XRCC1).

Because Rad9 possesses two BRCT domains, these domains may function in homodimer formation, heterodimer formation, and/or direct DNA binding, as has been 
demonstrated for other BRCT-containing proteins. Indeed, Rad9 has been shown to homodimerize through its BRCT domains, particularly in response to DNA damage (Soulier \& Lowndes, 1999). Specific point mutations of Rad9's BRCT domains (rad9$\mathrm{BRCT}^{\mathrm{MutF}+\mathrm{W}}$ ) does not allow Mec1-dependent hyperphosphorylation of Rad9 after DNA damage, nor is Rad53 activated, which is necessary to elicit a cellular arrest response (Soulier \& Lowndes, 1999). Moreover, this Rad9 BRCT mutant protein is abrogated in its ability to homodimerize, suggesting that $\operatorname{Rad} 9$ homodimerization via the BRCT domains contributes to $\operatorname{Rad} 9$ phosphorylation and activation of $\operatorname{Rad} 53$ and, therefore, to checkpoint arrest and repair (Soulier \& Lowndes, 1999). The possibility that Rad9's BRCT domains affect its association or heterodimerization with another protein needed for checkpoint activation still exists, as does the possibility that $\operatorname{Rad} 9$ can directly recognize and bind to DNA damage via its BRCT domains. Also unresolved is whether Mec 1-dependent $\operatorname{Rad} 9$ hyperphosphorylation is necessary to induce $\operatorname{Rad} 9$ homodimerization or whether homodimerization precedes Mec1-dependent hyperphosphorylation. More direct and rigorous tests of Rad9 homodimerization are provided herein.

To better characterize the BRCT domains in $\operatorname{Rad} 9$, we investigated the effects of specific Rad9 mutations. We show that removal of the BRCT domains ( $\operatorname{rad} 9 \triangle \mathrm{BRCTs})$ completely abrogates Rad9's ability to activate Rad53 and Chk1, in accord with disruption of arrest and DNA repair in response to damage. However, complete function could be restored by either overexpressing the rad9 $\triangle B R C T S$ allele or by forcing the 
protein to artificially dimerize by fusion to the GST protein ( $r a d 9 \Delta B R C T s-G S T)$. In vivo analysis of cell cycle arrest ( $\mathrm{S}-$ and $\mathrm{G}_{2} / \mathrm{M}$ phases) and DNA damage resistance (UV and MMS) was performed, along with biochemical analysis of Rad9 homodimerization and activation of the downstream kinases, Rad53 and Chk1. Preliminary coimmunoprecipitation results suggest that dimerization of $\operatorname{Rad} 9$ is not rate-limiting for function, as was previously thought. Additional genetic analysis of point mutations within Rad9's BRCT domains indicates that the BRCT repeats may affect proper protein localization or may negatively regulate $\operatorname{Rad} 9$ function. In total, our data demonstrates that the BRCT domains are predominantly required for Rad9 homodimer formation and protein stabilization, and we deduce that the dimerized state is required for the local concentration of $\operatorname{Rad} 9$ at sites of damage to facilitate $\operatorname{Rad} 9$-dependent reactions. In addition, $\operatorname{Rad} 9$ may be subject to negative regulation, which includes the possibility of regulation of protein localization, under certain cellular conditions to prevent checkpoint activation. 


\section{RESULTS}

\section{Expression of rad9 $\triangle B R C T$ s results in DNA damage sensitivity.}

To assess the contribution of the BRCT domains to Rad9 DNA damage checkpoint functions, we deleted these domains and analyzed the impact of their loss on cell phenotypes by expressing the $\operatorname{rad} 9 \triangle \mathrm{BRCT}$ s protein in cells subjected to DNA damage (Figure 3-1a). DNA damage of cells included either UV irradiation or inactivation of proteins encoded by the temperature-sensitive $c d c 13-1$ allele, which results in loss of $\mathrm{Cdcl} 3$ at telomere ends and leaves them prone to exonucleolytic degradation (Lydall \& Weinert, 1995). Whereas wild-type RAD9 expression resulted in a robust cell cycle arrest after $c d c 13$-1-induced damage, expression of the rad9 $\triangle B R C T s$ truncation under the $R A D^{9}$ promoter yielded no such arrest response (Figure 3-1b). Likewise, the wild-type $R A D 9$ strain was able to maintain high cell viability after UVdamage treatment, but the rad9 $\triangle B R C T s$ strain again exhibited a null response for repair of UV-induced damage (Figure 3-1c). Curiously, the abundance of rad9 $\triangle B R C T$ s protein is severely diminished in comparison with wild-type Rad9 protein levels, though this has no apparent bearing on rad9 $\triangle B R C T$ s functions (discussion follows). Therefore, under control of the $R A D 9$ promoter, Rad9's BRCT domains are necessary to initiate a cell cycle checkpoint arrest in the presence of damage and for repair UV-induced DNA lesions. 
1a)

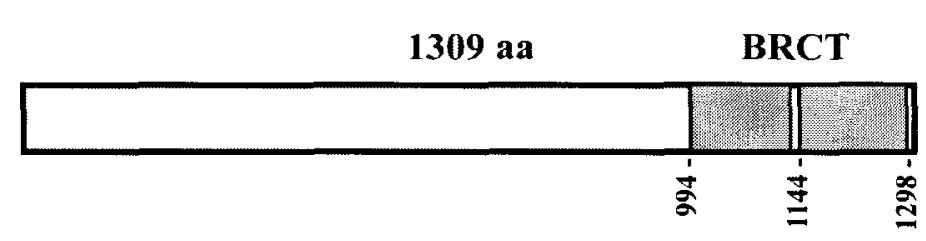

b)

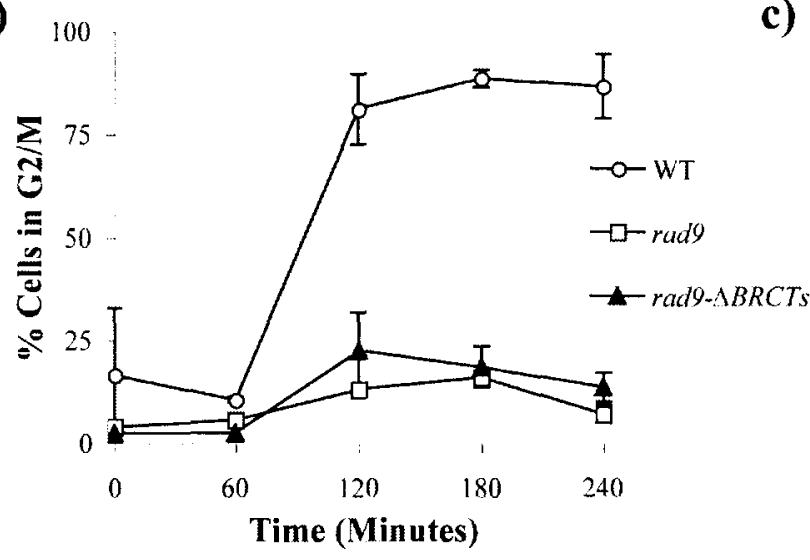

c)

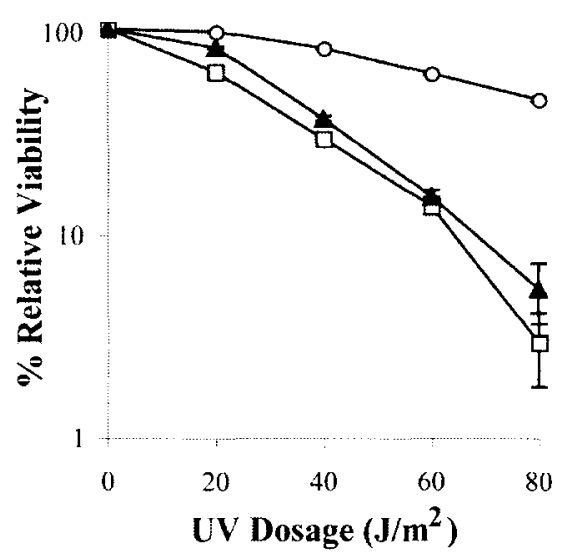

Figure 3-1. A map of the Rad9 protein and phenotypic effects of deleting the BRCT domains. (a) A map of the Rad9 protein sequence showing locations of the BRCT domains. (b) An assay for cell cycle arrest function of the rad9 $\triangle B R C T s$ allele. Midlogarithmic cells were synchronized in $\mathrm{G}_{1}$ with $\alpha$-factor at $23^{\circ} \mathrm{C}$. Cells were then released at the restrictive temperature $\left(36^{\circ} \mathrm{C}\right)$ to inactivate $c d c 13-1$ and induce DNA damage. Cells were fixed at various time points and subsequently scored for cell cycle position by analysis of DAPI-stained nuclei with fluorescent microscopy. (c) Assessing UV sensitivity of the rad $9 \triangle B R C T s$ allele. Mid-logarithmic cells were plated to solid media, exposed to various doses of UV irradiation, and allowed to grow overnight at $23^{\circ} \mathrm{C}$. Cells were then scored the following day for the ability to form microcolonies, indicative of UV resistance. For the cellular assays, each strain was tested in duplicate. The average of the duplicates is shown above, with error bars indicating the standard deviation from the mean.

Overexpression of rad $9 \triangle \mathrm{BRCTs}$ confers DNA damage resistance.

Using a different means to determine the contribution of the BRCT domains to Rad9 functions, we compared the effects of overexpressing Rad9 versus two Rad9 fragments - one consisting of Rad9 lacking the BRCT domains (rad9 $\Delta B R C T s)$ and the other comprised solely of the BRCT repeats (BRCTs). To this end, we created plasmid 
overexpression constructs, where our various $R A D 9$ alleles were placed under the control of the Gal promoter. When placed in galactose-containing media, the overexpression constructs produced a dramatic increase in protein levels as compared to levels of $\operatorname{Rad} 9$ protein expression under control of the normal promoter (Fig. 2a). We found the wildtype $\operatorname{Rad} 9$ protein to be overexpressed over 500 -fold above normal $\operatorname{Rad} 9$ protein levels, while the rad $9 \triangle B R C T$ s protein was subject to a much more moderate 25 -fold induction above wild-type Rad9. The diminished overexpression of the rad9 $\Delta \mathrm{BRCTs}$ protein suggests that the BRCT domains may play a role in stabilizing wild-type $\operatorname{Rad} 9$ proteins.

To analyze the contribution of the BRCT domains to Rad9-mediated damage resistance in strains overexpressing the truncated $\operatorname{rad} 9 \Delta B R C T$ s protein, strains were again assayed for their ability to withstand DNA damage caused by UV insult and for the ability to arrest at the $\mathrm{G}_{2} / \mathrm{M}$ checkpoint in response to $c d c 13-1$-induced damage. In sharp contrast to what we found when expressing $\operatorname{rad} 9 \triangle \mathrm{BRCT}$ from the $R A D 9$ promoter, overexpressing the rad $9 \Delta \mathrm{BRCTs}$ protein in $\mathrm{rad}^{-}$cells fully restored the resistance to $\mathrm{UV}$ damage (Figure 3-2b) and almost fully restored the DNA damage-dependent cell cycle arrest (Figure 3-2c). Overexpression of the BRCTs alone, however, had no effect, as this strain exhibited a rad9-phenotype. Because the overexpressed rad9 $\triangle B R C T$ s construct produced nearly wild-type phenotypic responses, these data suggest that the BRCT repeats are not required per se for cell cycle arrest in the presence of damage or repair of UV damage when the Rad9 $\mathrm{N}$-terminus is present at increased levels 25 times that of normal levels. 
2a)

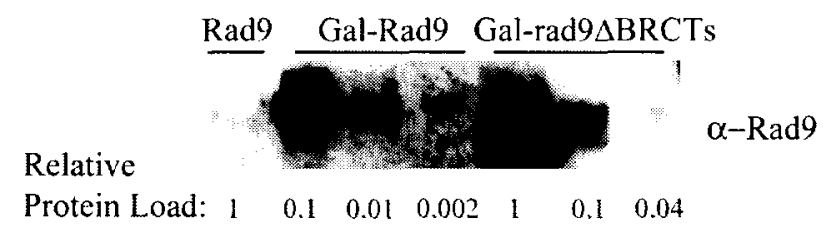

b)

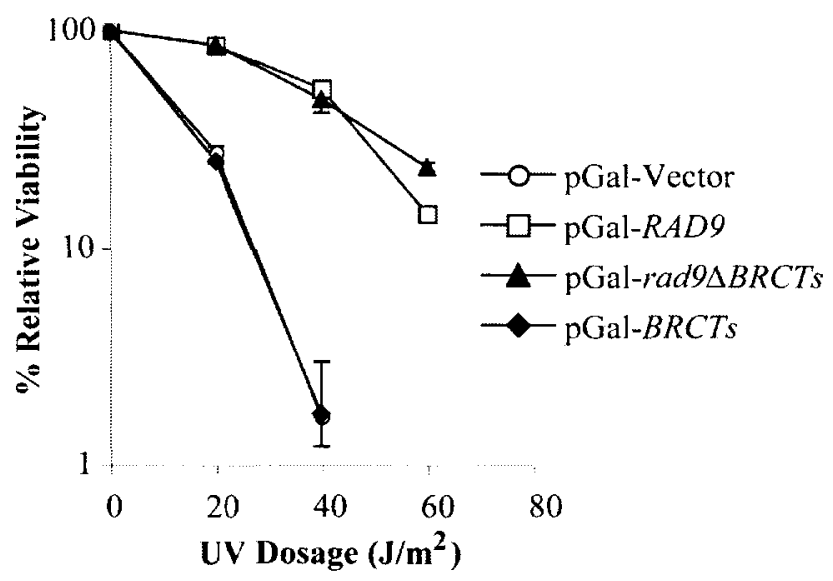

c)

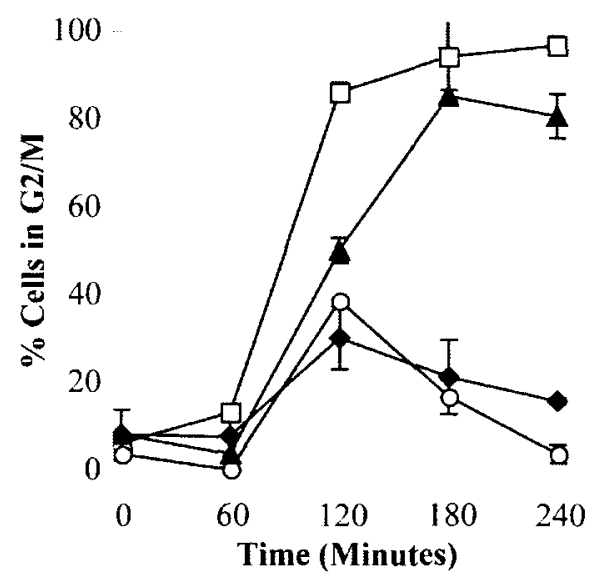

Figure 3-2. Overexpression levels of the rad9 $\triangle \mathrm{BRCT}$ protein and phenotypic analysis. (a) Mid-logarithmic cells were grown in the presence of 3\% galactose for 3 hours before being lysed in $20 \%$ TCA. For each sample, the same density of cells was lysed for protein analysis. Serial dilutions of proteins are shown above, with the relative loads indicated underneath each lane. An $\alpha$-Rad9 antibody (gift from D. Sterns) was used for detection of $\operatorname{Rad} 9$ protein. (b) For determination of UV sensitivity, mid-logarithmic cells were plated to minimal media, subjected to UV irradiation, and grown overnight. Cells were scored the following day for microcolony formation. (c) To determine cell cycle arrest proficiency, cells were arrested in $G_{1}$ with $\alpha$-factor and then released at the restrictive temperature $\left(36^{\circ}\right)$ to induce $c d c 13-1$ damage. Cells were fixed at various time points and scored for cell cycle position using fluorescent microscopy of DAPI-stained nuclei. For $(b)$ and $(c)$, each strain was assayed in duplicate. The average of the duplicates is shown with error bars representing standard deviations from the mean. 
Overexpression of the BRCTs alone does not cause a dominant-negative effect.

Although overexpression of just the BRCT domains produced no phenotype in a rad $9^{-}$background, we hypothesized that overexpression of the BRCTs in a $R A D 9^{+}$ background may result in a dominant-negative phenotype because $\operatorname{Rad} 9$ is known to homodimerize via its BRCT domains, and it is hypothesized that dimerization is required for checkpoint function (Soulier \& Lowndes, 1999). By this reasoning, we predicted that a wild-type $R A D 9^{+}$strain overexpressing the BRCTs fragment would become sensitized to DNA damage insult because the free BRCT repeats would dimerize with the BRCT domains in the endogenous $\operatorname{Rad} 9$ proteins to render them non-functional. To assess any dominant-negative effects, we independently overexpressed the BRCTs protein and the $\operatorname{rad} 9 \triangle \mathrm{BRCT}$ s protein in a wild-type $R A D 9^{+}$background and then subjected the cells to DNA damage. Interestingly, all strains showed a wild-type response for both arrest proficiency and UV resistance, and, therefore, caused no dominant-negative effect (data not shown).

Overexpression of Rad9 or $\operatorname{rad} 9 \triangle B R C T$ s does not cause constitutive arrest.

To determine if high protein levels of $\operatorname{Rad} 9$ or $\operatorname{rad} 9 \Delta \mathrm{BRCT}$ s could induce a constitutive arrest response in the absence of DNA damage, the proteins were overexpressed in a $\mathrm{CDCl} 3^{+}$strain. Cells were fixed over time and DAPI-stained cell nuclei were analyzed for cell cycle position. No such constitutive arrest was seen when overexpressing Rad9 or $\operatorname{rad} 9 \Delta B R C T s$ (data not shown), leading us to infer that a Rad9activating event must precede arrest - presumably recruitment of other checkpoint 
proteins (e.g., $M e c 1, \operatorname{Ddc} 2, \operatorname{Rad} 17, \operatorname{Rad} 24$, etc.) to sites of DNA damage that then, in turn, leads to Rad9 phosphorylation.

Restoration of wild-type function of $\operatorname{rad} 9 \triangle B R C T$ s by forced dimerization.

Given that Rad9 was previously shown to homodimerize via its BRCT domains, and based on our data that overexpression of the $\operatorname{rad} 9 \Delta \mathrm{BRCT}$ s protein obviated the need for the BRCTs in arrest and UV damage responses, we reasoned that the BRCT domains might be necessary to increase local concentrations of Rad9 for function by homodimerization. Our data support a model whereby under normal levels of expression, a $R A D 9$ allele lacking the BRCT repeats does not allow for homodimerization and, consequently, insufficient local concentration of Rad9 is achieved for checkpoint function, accounting for the null phenotype of the rad9 $\triangle B R C T s$ strain. Overexpression of $\operatorname{rad} 9 \triangle \mathrm{BRCTs}$, however, circumvents the need for local concentration through homodimerization by increasing protein concentrations enough within the cell to carry out checkpoint functions.

To determine if dimerization via the BRCT domains is necessary for $\operatorname{Rad} 9$ function under normal expression levels of protein, we artificially forced dimerization of $\operatorname{rad} 9 \triangle \mathrm{BRCT}$ s to see if this would restore function. To force dimerization, we used the GST protein, which has been reported in the literature to form dimers and tetramers (Ladbury et al., 1995; Walker et al., 1993; Ji et al., 1992; Parker et al., 1990). Moreover, the GST protein has been proven to be a useful substitute for the oligomerization domains 
3a)

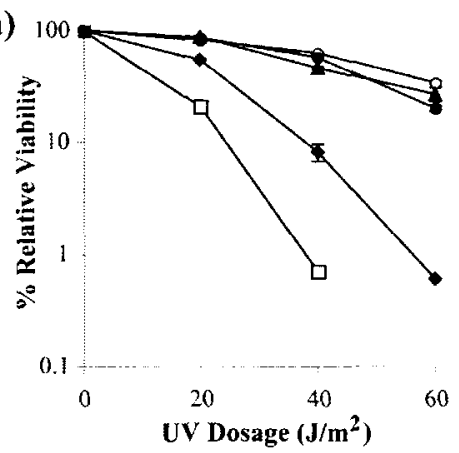

c)

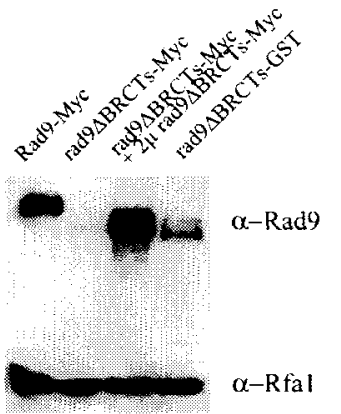

e)

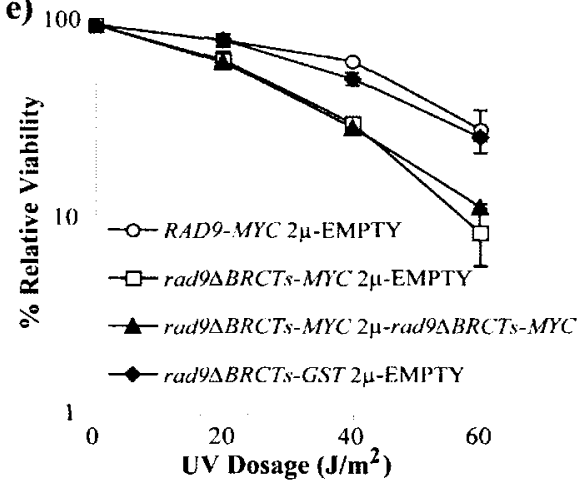

b) 100

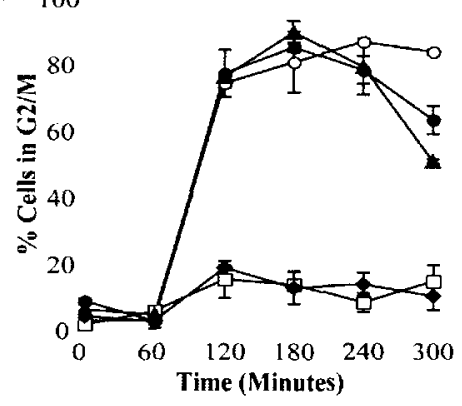

d)

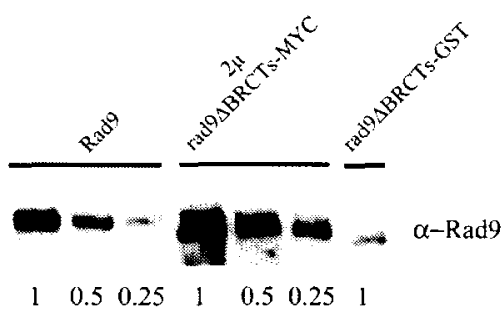

f)

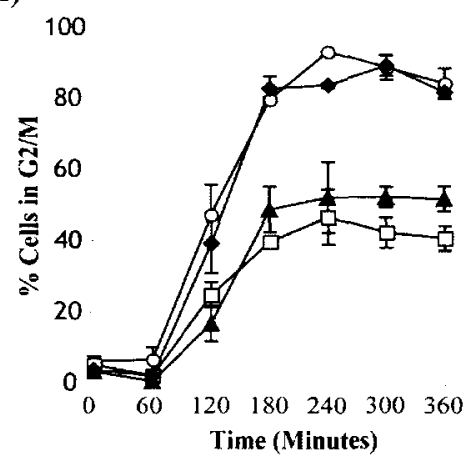

Figure 3-3. Characterization of strains containing non-dimerizing rad9 $\triangle B R C T s-M y c$ and dimerizing rad9 $\triangle B R C T s-G S T$ proteins. (a) UV sensitivity, $(b)$ arrest proficiency, $(c)$ and protein expression levels of strains containing $\operatorname{Rad} 9$ and truncated proteins under normal levels of expression. (d) Comparison of protein expression levels between endogenously expressed Rad9, truncated rad9 $\triangle B R C T s-G S T$, and the rad9 $\triangle B R C T s-M Y C$ strain carrying a $2 \mu$ multi-copy plasmid containing the $\operatorname{rad} 9 \triangle B R C T s-M Y C$ sequence. Serial dilutions of protein are shown with the relative loads indicated underneath. The same volume of cells was assayed for protein extraction. (e) UV sensitivity and $(f)$ arrest proficiency of strains containing either a $2 \mu$ plasmid empty vector or the $2 \mu$ rad $9 \Delta B R C T s-M Y C$ plasmid. Cell cycle assays were performed as previously described. Each strain was assayed in duplicate. The average value of the duplicates is shown with error bars representing the standard deviation from the mean. 
of heterologous proteins (Maru et al., 1996; Riley et al., 1996; Maru, 2000), and can also be used to dimerize a protein of interest that does not oligomerize under normal conditions (Tudyka \& Skerra, 1997).

When the GST protein was placed at the C-terminal end of the rad9 $\triangle \mathrm{BRCTs}$ protein ( $\operatorname{rad} 9 \triangle \mathrm{BRCTs}-\mathrm{GST})$ expressed from the $R A D 9$ promoter, wild-type function for arrest and UV resistance was restored (Fig. 3-3a, b). Conversely, placement of the Myc epitope at the C-terminal end of this protein (rad9ABRCTs-Myc) did not restore either UV resistance or arrest function, as these strains demonstrated nearly null phenotypes under normal levels of expression.

In looking at protein expression levels, we found the rad9 $\triangle \mathrm{BRCTs}-\mathrm{Myc}$ and the $\operatorname{rad} 9 \Delta B R C T s-G S T$ proteins to be expressed at levels less than that of wild-type Rad9, with rad9 $\triangle$ BRCTs-Myc expression being even less than that of rad9 $\triangle$ BRCTs-GST (Fig. 3-3c, d). Therefore, to determine whether the null phenotype for arrest and the hypomorphic UV-resistance phenotype associated with the rad9 $\triangle B R C T s-M Y C$ strain were due to low protein levels or the lack of BRCT domain functions, we boosted $\operatorname{rad} 9 \Delta \mathrm{BRCTs}-\mathrm{Myc}$ expression by placing it in a multi-copy $2 \mu$ plasmid. While the multicopy plasmid restored rad $9 \Delta \mathrm{BRCTs}-\mathrm{Myc}$ protein expression to levels approximately twice that of wild-type Rad9 (Figure 3-3d), the null phenotypes were not complemented by the increase in protein dosage (Figure 3-3e, f). (Of note, the arrest response of the rad $9 \triangle B R C T s-M Y C 2 \mu$-rad $9 \triangle B R C T s-M Y C$ strain does increase, but it also increases in 
the $\operatorname{rad} 9 \triangle B R C T s-M Y C$ strain carrying the $2 \mu$ empty vector. We attribute this apparent increase in arrest to different growth conditions - minimal media versus rich media - that might affect cell cycle kinetics.) These data indirectly indicate that dimerization of the Rad9 N-terminus, whether via the C-terminal BRCT domains or another dimerizing domain such as the GST protein, is critical for $\operatorname{Rad} 9$ checkpoint functions. Moreover, because the dimerizing GST protein can completely complement loss of the BRCT domains, this suggests that the sole role of the BRCTs in DNA damage arrest and UV resistance is to support dimerization of the $\operatorname{Rad} 9 \mathrm{~N}$-terminus.

\section{Direct tests to confirm rad9 $\triangle B$ BCTs-GST constitutively dimerizes.}

We infer the rad9 $\triangle B R C T S$-GST strain to be wild-type for cell cycle arrest and UV repair responses because the GST protein is forcing dimerization of the Rad9 N-termini a state necessary for interaction with the downstream checkpoint component $\operatorname{Rad} 53$ and perhaps also with Chk1. To verify dimerization of $\operatorname{rad} 9 \Delta \mathrm{BRCTs}$ proteins caused by GST homodimer interactions, we created strains with differentially tagged rad9 $\mathrm{BRCT}$-GST proteins - rad9 $\triangle B R C T-G S T-M Y C$ and $\operatorname{rad} 9 \triangle B R C T-G S T-H A$ - so that coimmunoprecipitations could be done. The dually tagged rad9 $\triangle B R C T$-GST strain, along with dually tagged positive and negative control strains (RAD9-MYC/RAD9-HA and rad9 $\triangle B R C T s-M Y C / r a d 9 \triangle B R C T s-H A$, respectively), were treated with DNA damage and tested for the state of dimerization by Western blot analysis. 
Despite several different approaches and numerous attempts, I have not been able to obtain clear co-immunoprecipitation data. Rad9 is incredibly sensitive to protein degradation upon cell lysis and it is also not readily soluble, making it difficult to immunoprecipitate a high yield of full-length protein. Also, in our hands, the HA epitope produces a very weak signal by antibody detection, thus compounding co-

immunoprecipitation problems further. Efforts are currently underway to utilize the highaffinity TAP epitope tag in place of the HA epitope in order to complete the coimmunoprecipitation experiments (Rigaut et al., 1999).

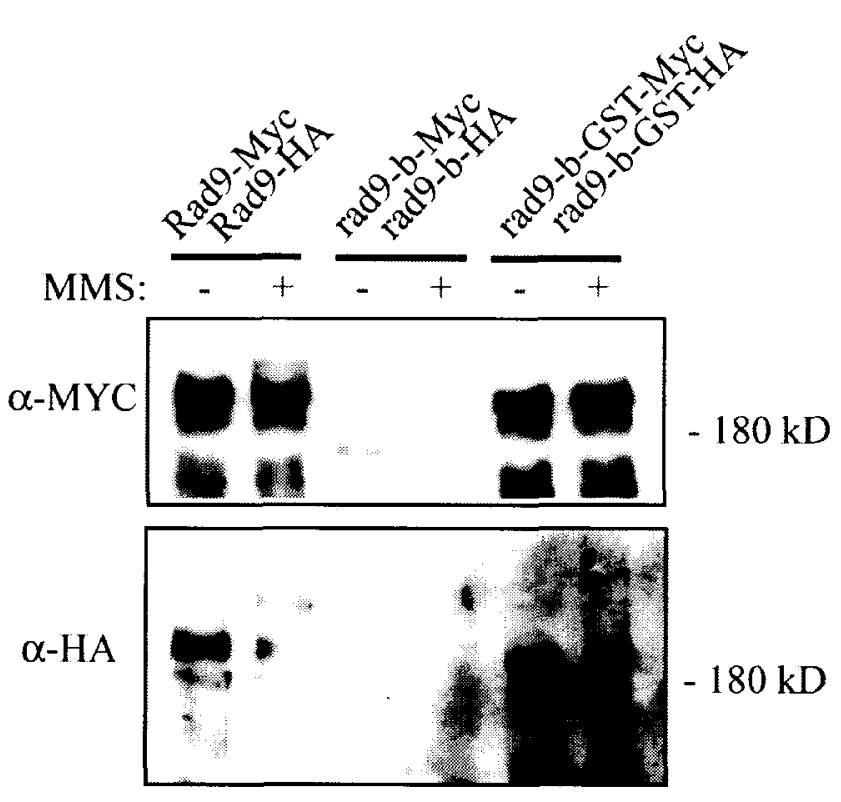

Figure 3-4. Co-immunoprecipitation of epitope-tagged Rad9 and rad9 $\Delta B R C T s$ proteins. Diploid cells containing dually tagged $\operatorname{Rad} 9$ and rad9 $\triangle B R C T$ s proteins expressed under control of the normal promoter were grown to the same cell density in untreated liquid media or media containing $0.1 \% \mathrm{MMS}$ to induce DNA damage. Cells were then lysed and immunoprecipitated with $\alpha$-MYC sepharose beads. Immunoprecipitated extracts were separated on a $6 \%$ acrylamide gel, transferred to a nitrocellulose membrane, and probed with $\alpha$-Myc and $\alpha$-HA antibodies. The full-length and truncated Rad9 proteins run slightly higher than the $180 \mathrm{kD}$ marker indicated to the right of the Western blots. 
The results shown in Figure 3-4 demonstrate the Western detection signals that I am able to attain. By pulling down Myc-tagged Rad9 or rad9 $\Delta B R C T$ s fragments, I am able to co-immunoprecipitate the corresponding HA-tagged protein in both the wild-type strain containing full-length $\operatorname{Rad} 9$ and the in the strain containing the GST-tagged $\operatorname{rad} 9 \triangle \mathrm{BRCTs}$ fragment under both non-DNA-damaging and DNA-damaging conditions. No HA-tagged rad9 $\triangle$ BRCTs appears in the co-immunoprecipitated Western blot for the $\operatorname{rad} 9 \triangle \mathrm{BRCTs}$ protein that lacks the GST fusion. While this is likely due to the inability of the protein to dimerize, such a statement cannot be definitively made since the protein levels present in the $\alpha-\mathrm{Myc}$ Western blot are so low. If any co-immunoprecipitated HAprotein is present in the $\alpha$-HA blot, it is probably undetectable by my methods given the low protein abundance. Though these blots are far from publishable, I am convinced by the data presented therein - namely that wild-type Rad9 dimerizes with and without DNA damage induction and that the rad $9 \triangle \mathrm{BRCTs}$-GST protein is constitutively dimerized, as expected. Publishable co-immunoprecipitation results that would directly confirm that the GST protein restores dimerization to the $\operatorname{rad} \triangle \mathrm{BRCT}$ s protein are still required.

Determining if Rad9 is constitutively dimerized irrespective of DNA damage. The observation that $\operatorname{Rad} 9$ readily dimerizes irrespective of DNA damage is contrary to a previous hypothesis. Soulier \& Lowndes (1999) showed that a fraction of Rad9 interacts with itself in undamaged cells, but that after damage induction, Rad9 molecules demonstrated a greater affinity for dimerization. Furthermore, this dimerization was shown to be dependent on the BRCT domains, as it was abrogated in a 
strain with point mutation of highly conserved aromatic residues within these domains.

Because the data depicted in Figure 3-4 was obtained using strains with the temperaturesensitive $c d c 13-1$ mutation in the background, it is formally possible that these cells undergo a low level of endogenous damage at telomeres even though they are grown at the permissive temperature. The possibility of low levels of $c d c 13-1$ damage may account for the Rad9 dimerization I see when cells are not treated with MMS damage. To confirm if $\operatorname{Rad} 9$ does indeed constitutively dimerize regardless of DNA damage presence, I have created and introduced a $C D C 13^{+}$centromeric plasmid into the strains assayed in Figure 3-4 to eliminate any possible endogenous $c d c 13-1$ damage. Based on my crude results, it does indeed appear that $\operatorname{Rad} 9$ is constitutively dimerized irrespective of NDA damage (Figure 3-5). Upon better co-immunoprecipitation methods, these strains will be better assayed for dimerization with and without MMS treatment in the presence of $C D C 13^{+}$.

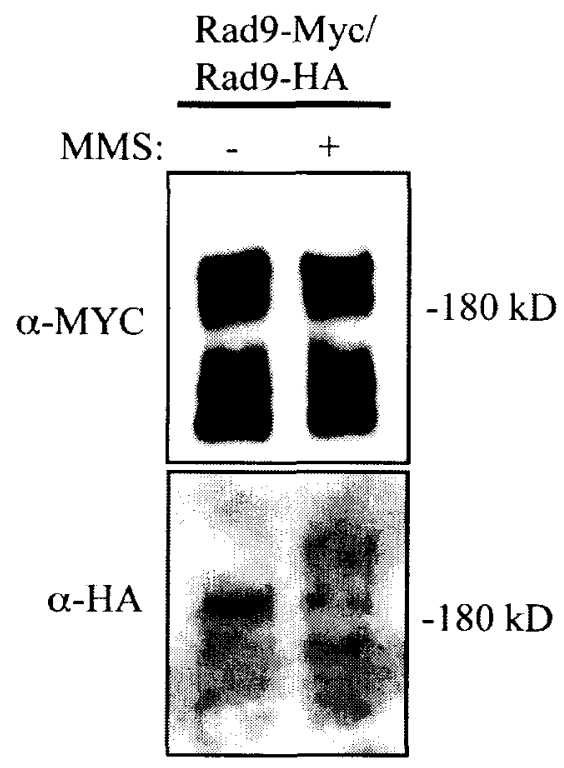

Figure 3-5. Co-immunoprecipitation of epitope-tagged Rad9 in a strain containing CDC13. Diploid cells containing dually tagged Rad9 and a $C D C 13^{+}$plasmid were grown in liquid media. Cells were either left untreated or treated with $0.1 \%$ MMS to induce DNA damage. Cells were then treated as described in Figure 3-4. 
Activation of downstream kinases by rad9 $\triangle B R C T s-G S T$.

Dimerization of Rad9, whether via the BRCTs or the GST protein, correlated with its phosphorylation in response to DNA damage, its activation and interaction with Rad53, and its activation of Chk1. The wild-type and rad9 $\triangle B R C T s-G S T$ proteins displayed hyperphosphorylation in response to $c d c 13-1$ and MMS-induced damage, whereas the rad9 $\triangle B R C T s-M y c$ construct was not substantially phosphorylated (Figure 36). Furthermore, phosphorylation of the dimerized proteins correlated with the ability of the proteins to interact with phosphorylated Rad53. That is, the rad9 $\triangle B R C T s-G S T$ protein was able to activate and co-immunoprecipitate phosphorylated $\operatorname{Rad} 53$ in the presence of $c d c 13-1$ - and MMS-induced damage as well as did wild-type Rad9, however

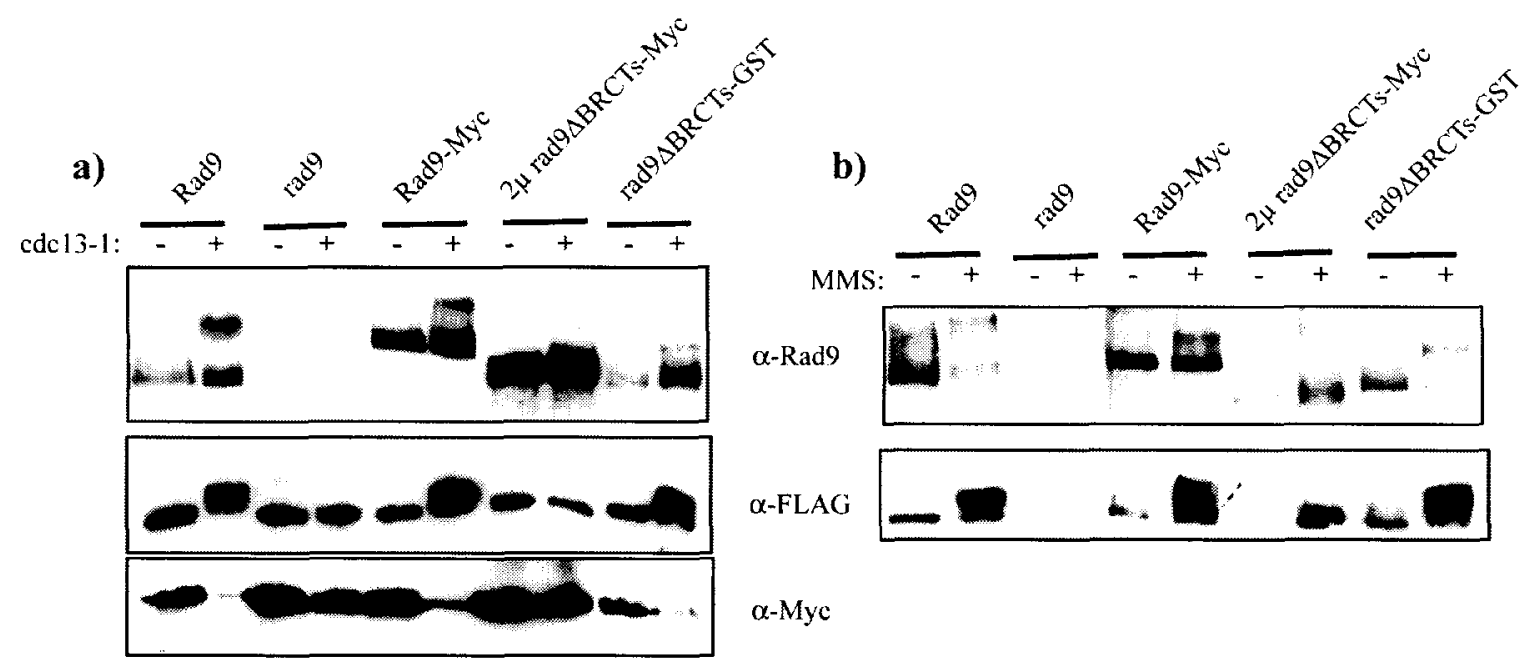

Figure 3-6. Activation of downstream kinases by dimerizing rad $9 \Delta \mathrm{BRCTs}-\mathrm{GST}$ but not non-dimerizing rad9 $\triangle \mathrm{BRCTs}-\mathrm{Myc}$. (a) Total protein extracts were prepared by $20 \% \mathrm{TCA}$ precipitation from cells either grown normally or subjected to $c d c 13-1$ damage for 3 hours at the restrictive temperature. (b) The top panel shows immunoprecipitation of $\operatorname{Rad} 9$ using $\alpha-\operatorname{Rad} 9$ antibodies. The panel below shows co-immunoprecipitated Rad53FLAG. TCA and IP Westerns were probed with either $\alpha$-Rad9, $\alpha$-FLAG antibody to detect Rad53-FLAG, or $\alpha$-Myc to detect Chk1-Myc. 
the non-dimerizing rad9 $\Delta \mathrm{BRCTs}-\mathrm{Myc}$ protein was unable to do so (Figure 3-6b). Similarly, Chk1 was activated in both the wild-type and rad9ABRCTs-GST strain, whereas no Chk1 phosphorylation was evident in the non-dimerizing rad9 $\triangle B R C T s-M Y C$ strain (Figure 3-6a).

rad $9 \triangle B R C T s-G S T$ demonstrates cell cycle arrest kinetics identical to that of $R A D 9$. Because the rad9 $\triangle B R C T s-G S T$ strain was virtually indistinguishable from the wild-type $R A D 9$ strain in the arrest and UV resistance assays, we sought to identify some difference in phenotype between the two strains, which might indicate some role of the BRCT domains above and beyond that of dimerization. In accord with the prevailing model, if Rad9 dimerizes only after DNA damage, we hypothesized that the constitutively-dimerized $\operatorname{rad} 9 \Delta \mathrm{BRCTs}$-GST protein might render cells more responsive to DNA damage than wild-type cells by having already overcome the need to dimerize for function in the checkpoint cascade. To test this, cell cycle arrest was determined when cells were shifted to the semi-permissive temperatures of $28^{\circ} \mathrm{C}$ and $26.5^{\circ} \mathrm{C}$ to partially inactivate the temperature-sensitive $\operatorname{cdc} 13$ protein (the restrictive temperature for $c d c 13-1^{t s}$ strains is $>28^{\circ} \mathrm{C}$ ). No difference was seen between the percent of cells that arrested for either strain at either temperature (data not shown).

We also hypothesized that the $\operatorname{rad} 9 \triangle B R C T S$-GST strain may remain arrested for longer after damage insult if dissociation of the dimer was required to resume cell cycle progression, analogous to $\operatorname{Rad} 9$ dephosphorylation that is required for resuming cell cycle 
progression after damage (Pellicioli et al., 2001; Esashi \& Yanagida, 1999). Cells were thus incubated at the restrictive temperature to induce arrest and then shifted down to the permissive temperature to allow cells to resume cycling. Again, no difference was seen in the amount of $R A D 9$ and rad $9 \triangle B R C T s-G S T$ cells that arrested, and both strains resumed cell cycling by proceeding through mitosis at the same time and at the same rate (data not shown).

Both results - that $R A D 9$ and $\operatorname{rad} 9 \triangle B R C T s-G S T$ are similarly sensitive to DNA damage and that they show the same kinetics of cell cycle progression after DNA damage induction and repair - are consistent with possibility that Rad9 is actually constitutively dimerized, as the rad9 $\triangle \mathrm{BRCTs-GST}$ proteins are. Alternatively, it is possible that $\operatorname{Rad} 9$ dimerization is not rate-limiting for protein function. Regardless, these results lend credence to the ability of the dimerizing GST protein to completely restore rad9 $\triangle B R C T s$ function by replacement of the BRCT domains and further support the hypothesis that the predominant role for the BRCTs in $\operatorname{Rad} 9$ is to mediate protein dimerization.

rad9 $\triangle B R C T s-G S T$ causes the same adaptation response as that of $\operatorname{Rad} 9$.

Cells constitutively assaulted with DNA damaging conditions arrest for about eight hours before undergoing adaptation, or resumption of cell cycle progression in the presence of DNA damage (Sandell \& Zakian, 1993). This process appears to require the dephosphorylation of Rad9 to down-regulate the checkpoint cascade (Pellicioli et al., 2001; Sanchez et al., 1999), but whether the state of Rad9 dimerization must be altered 
for adaptation to occur is unknown. In accord with the model whereby Rad9 predominantly dimerizes upon damage induction, we sought to determine if dissociation of Rad9 dimers is required for adaptation, again, to discern a difference between $R A D 9$ and rad $9 \triangle B R C T s$ - $G S T$ strains. $R A D 9$ and $\operatorname{rad} 9 \triangle B R C T s$ - $G S T$ cells were incubated at the restrictive temperature, separated into individual large-budded cells on solid, rich media by micromanipulation, and maintained at the restrictive temperature for over eight hours. The individual large-budded cells were then analyzed for the presence of additional cell buds, indicative of adaptation. We found that $85.4 \%$ (41/48) of wild-type cells adapted and $83.3 \%(45 / 54)$ of $\mathrm{rad} 9 \triangle B R C T s$-GST cells adapted, thereby disfavoring the possibility that dissociation of Rad9 dimers is needed for adaptation. This point indirectly lends support to the notion that Rad9 may be constitutively dimerized, like $\operatorname{rad} 9 \Delta \mathrm{BRCTs}-\mathrm{GST}$, since no phenotypic difference was seen between the two strains analyzed. Alternatively, dissociation of $\operatorname{Rad} 9$ dimers may not be a key factor that determines the ability to adapt; perhaps, rather, the state of phosphorylation is a key determinant in the adaptation response, for which both proteins are similarly affected.

The role of $\operatorname{Rad} 9 \& \operatorname{rad} 9 \triangle \mathrm{BRCT}$ s in response to S-phase damage.

While the role of $\operatorname{Rad} 9$ at the $\mathrm{G}_{2} / \mathrm{M}$ cell cycle transition has been well characterized, its role during S-phase remains unclear. Whereas Rad9 is essential for a checkpoint response during $\mathrm{G}_{2} / \mathrm{M}$ in the presence of DNA damage, $\operatorname{rad} 9$ mutation causes only a weak increase in sensitivity of cells treated with MMS, an agent that produces DNA adduct addition causing wild-type cells to slow the rate of replication (Lopes et al., 
2001; Tercero \& Diffley, 2001; Paulovich \& Hartwell, 1995). Futhermore, rad9 mutants demonstrate no appreciable sensitivity to hydroxyurea (HU), which depletes dNTP pools to slow the rate of S-phase replication. Still unresolved is whether Rad9 performs functions during S-phase that are different from those during $\mathrm{G}_{2} / \mathrm{M}$. While homodimerization via the BRCT domains may be required for specific Rad9 S-phase functions similar to those in G/M, such as interaction with Rad53, Rad9 could carry out additional S-phase roles by heterodimerizing with other proteins and/or by directly binding to DNA.

We used MMS to test the roles of our various deletion proteins in S-phase responses (Figure 3-7). These assays test both the requirement of the BRCT domains for Rad9 function in S-phase damaged cells and also the importance of $\operatorname{Rad} 9$ dimerization for these functions. The $R A D 9-M Y C$, rad9 $\triangle B R C T S-M Y C$, and rad9 $B B C T S-G S T$ strains were treated with $0.033 \%$ MMS over time and assessed for cell sensitivity. As can be seen in Figure 3-7a, both the $R A D 9-M Y C$ and $\operatorname{rad} 9 \triangle B R C T s-G S T$ strains are as MMS resistant as the wild-type $R A D 9$ strain. The $\operatorname{rad} 9 \triangle B R C T S-M Y C$ strain, on the other hand, is much more sensitive to MMS damage, though not as much as a rad9 null strain, similar to its degree of UV sensitivity that we saw previously. This hypomorphic MMS resistance must be due to the inability of the rad9 $\mathrm{BRCTs}-\mathrm{MYC}$ protein to homodimerize and not due to loss of some additional function mediated by the BRCT domains, such as heterodimeric interactions or DNA binding, since the homodimerizing rad9 $\triangle B R C T s-G S T$ strain also lacks the BRCT domains and the potential to 
a)

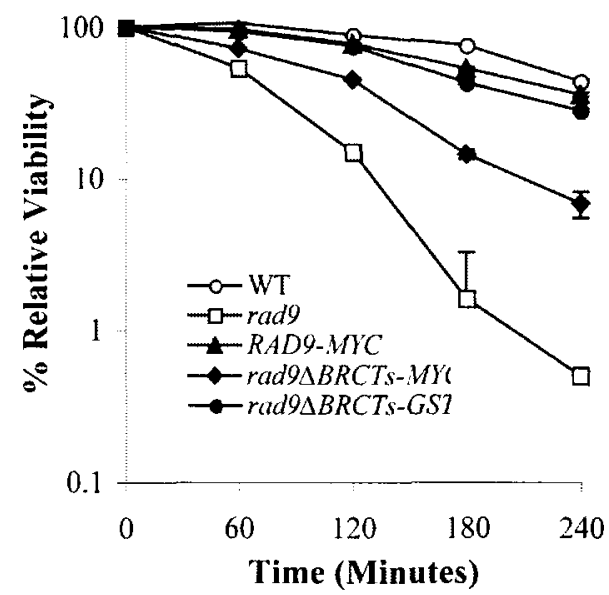

c)

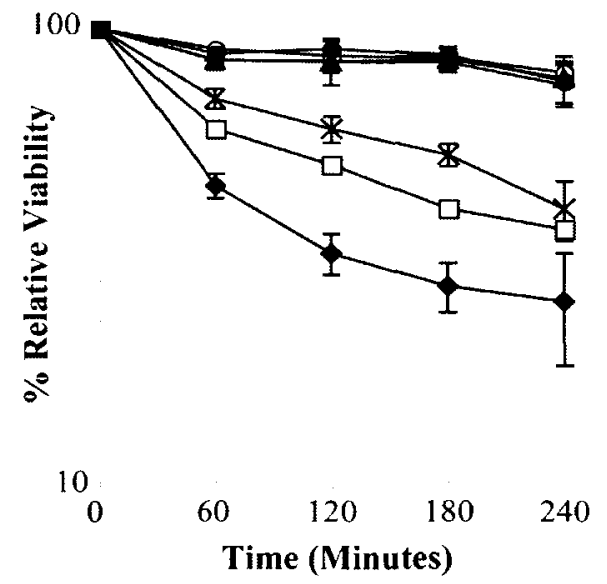

a-WT

b)

$\triangle-$ RAD9-MYC mrcl-

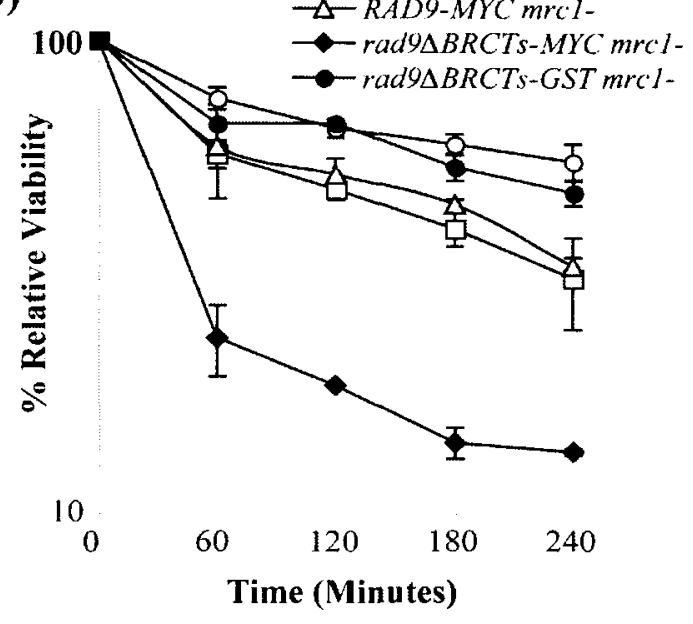

$\multimap$ WT

$\rightarrow-\operatorname{rad} 9-$

$\rightarrow$ tof 1 -

$\neg$ rad 9 - tofl-

$\rightarrow$ RAD9-MYC tof 1 -

* rad $9 \triangle B R C T s-M Y C$ tof 1 -

十 rad $9 \Delta B R C T s-G S T$ tofl-

Figure 3-7. S-phase responses of cells containing non-dimerizing rad9 $9 B R C T s-M y c$ and dimerizing rad9 $\triangle \mathrm{BRCTs-GST}$. (a) Mid-logarithmic liquid cultures were treated with $0.033 \% \mathrm{MMS}$ and plated to solid media at the times indicated. Cells were grown overnight at $23^{\circ} \mathrm{C}$ and scored the following day for microcolony formation. (b) MMS sensitivity of $\mathrm{mrcl}^{-}$strains was assayed as described in (a), except cells were treated with $0.00019 \%$ MMS. (c) MMS sensitivity of tof $1^{-}$strains was assayed as described in $(b)$. Duplicates of each strain were tested, and the average of the duplicates is shown above with error bars representing the standard deviation from the mean. 
heterodimerize or bind DNA but is wild-type for MMS resistance. Under the given conditions, these data indicate that in S-phase the BRCT domains are required for homodimerization to enable Rad9 function, implying that the role of Rad9 in S-phase is similar to its role in $\mathrm{G}_{2} / \mathrm{M}$.

Because Rad9 does not play a large role in the checkpoint response to damage during S-phase, it is hypothesized that other proteins function in the place of Rad9 in DNA damage recognition, processing, and/or repair during this phase of the cell cycle. Indeed, cells lacking Rad9 can be sensitized to MMS damage by elimination of either Tof1 or Mrc1, proteins thought to be partially redundant for Rad9 in mediating an Sphase checkpoint response to damage (Alcasabas et al., 2001; Foss, 2001). Hence, the rad9 tofl double mutant is as MMS sensitive as a mec1 mutant, and the rad9 mrcl mutant is synthetically lethal.

To enhance the Rad9-dependent S-phase response to damage, the TOFI and $M R C 1$ genes were knocked out in our various Rad9-tagged strains. The strains were again subjected to MMS damage and analyzed for cell sensitivity. The addition of the mrcl mutation slightly sensitizes $R A D 9, R A D 9-M Y C$, and $\operatorname{rad} 9 \Delta B R C T s-G S T$ strains to MMS damage compared to the wild-type $R A D 9 M R C 1$ strain (Figure 3-7b). Whereas a rad9 $\mathrm{mrcl}$ strain is synthetically lethal, the $\operatorname{rad} 9 \Delta B R C T s-M Y C \operatorname{mrcl}$ strain is viable but shows a more pronounced MMS sensitivity compared to the other strains tested. Likewise, addition of the tof 1 mutation has a similar, though lesser effect on cells (Figure 
3-7c). tofl in combination with $R A D 9, R A D 9-M Y C$, and rad9 $\triangle B R C T s-G S T$ is not appreciably sensitized to MMS damage, whereas the rad9 $B R C T s-M Y C$ strain show a greater sensitivity to MMS, though not as great as that of the rad9 tof 1 double mutant. These results indicate that the rad9 $\triangle \mathrm{BRCTs}$-GST protein is fully functional for S-phase functions and that the roles of $\operatorname{Rad} 9$ in S-phase look to be equivalent to its roles in $G_{2} / M$. Furthermore, because the rad $9 \Delta B R C T s-M Y C$ strain does retain some resistance to MMS damage, this suggests that some Rad9 function can be carried out without the function of the BRCT domains. What these functions entail remains to be determined.

Analyzing $R A D 9$ BRCT point mutations and the effects on protein function.

The BRCT domains fold to form a globular structure with a hydrophobic core. Within each domain is an aromatic residue - either a phenylalanine or a tryptophan - that is the most highly conserved feature in BRCTs across all species (Bork et al., 1997; Callebaut et al., 1997). Based on crystal structure, this residue lies at the center of the highly conserved hydrophobic pocket (Zhang et al., 1998). Mutation of these aromatic residues is thought to severely abrogate proper BRCT domain folding. The Breast Cancer Information Core (BIC) database reports the mutation of the conserved tryptophan of the second BRCT motif of BRCAl (W1708D) found in beast cancer tissue, indicating the severity of such a mutation (Soulier \& Lowndes, 1999). Another reported mutation within the second BRCA1 BRCT domain, W1837R, also identified in breast cancer tissue, causes a hydrophobic residue to be replaced by charged residue that would presumably prevent proper formation of the hydrophobic fold (Zhang et al., 1998). 
Point mutation of the highly conserved phenylalanine residue in Rad9's first BRCT domain (F1104A) and the tryptophan in the second BRCT domain (W1280A) were previously analyzed both singly ( $\mathrm{rad}^{M u t F}$ and $\mathrm{rad} 9^{M u t W}$ ) and in combination $\left(\operatorname{rad} 9^{M u t F+W}\right.$; Soulier \& Lowndes, 1999). It was shown that strains containing these mutated proteins are as sensitive to UV damage as a rad 9 null and also demonstrate cell cycle arrest defects in the presence of DNA damage. We also chose to assess the contribution of Rad9's BRCT domains using these point mutations. After confirming Soulier \& Lowndes' initial results, we reasoned that Gal overexpression of the $\operatorname{rad} 9^{\mathrm{MutF}+\mathrm{W}}$ point mutant might restore DNA damage and arrest functions as we found when we overexpressed the rad $9 \triangle B R C T$ s protein under control of the Gal promoter. An overexpression construct containing the $\operatorname{rad} 9^{\mathrm{MutF}+W}$ sequence was created and overexpressed in a rad $^{-}$background. Surprisingly, this strain was null in its ability to arrest in response to $c d c 13-1$ damage and in its ability to repair UV-induced DNA damage (Figure 3-8a, b). This result stands in contrast to that of overexpression of the $\operatorname{rad} 9 \Delta \mathrm{BRCTs}$ protein: $\operatorname{rad} 9 \Delta \mathrm{BRCT}$ s cannot dimerize via the BRCT domains presumably just like $\operatorname{rad} 9^{\mathrm{MutF}+\mathrm{W}}$, yet overexpression of $\operatorname{rad} 9 \Delta \mathrm{BRCT}$ s protein can rescue a $\operatorname{rad} 9^{-}$strain whereas overexpression of $\operatorname{rad} 9^{\mathrm{MutF}+\mathrm{W}}$ cannot. This implies that the $\operatorname{rad} 9^{\mathrm{MutF}+\mathrm{W}}$ expressing strain is defective in some way that the rad9 $\triangle B R C T s$-expressing strain is not. Overexpression of the $\operatorname{rad} 9^{M u t F+W}$ construct in a $R A D 9^{+}$background produced no dominant negative effect for either the ability to arrest or repair UV damage (data not shown). 
A possible explanation for the previous result is that $\operatorname{rad} 9^{\mathrm{MutF}+\mathrm{W}}$ still needs to dimerize for some reason in order to function. Given that the BRCT point mutations probably render Rad 9 non-functional by abrogating dimerization, we predicted that fusion of the GST protein to the C-terminus of $\mathrm{rad} 9^{\mathrm{MutF}+\mathrm{W}}$ might re-establish dimerization of the protein to restore its function, as it does for the $\operatorname{rad} 9 \Delta \mathrm{BRCTs}-\mathrm{GST}$ protein. We tested arrest in response to damage and UV repair in these strains and found that normal expression (under control of the $R A D 9$ promoter) of $\operatorname{rad} 9^{\mathrm{MutF}+\mathrm{W}}-\mathrm{GST}$, like both normal expression and overexpression of $\operatorname{rad} 9^{\mathrm{MutF}+\mathrm{W}}$, produced a null response (Figure 3-8c, d). Also, though the $\operatorname{rad} 9^{\mathrm{MutF}+\mathrm{W}}$ protein was as abundant as wild-type Rad9, it did not undergo hyperphosphorylation as Rad9 did in response to DNA damage (Figure 3-8e). Moreover, the $\operatorname{rad} 9^{\mathrm{MutF}+\mathrm{W}}-\mathrm{GST}$ protein did not become hyperphosphorylated as $\operatorname{rad} 9 \Delta \mathrm{BRCTs}-\mathrm{GST}$ did (Figure 3-6), though we are presumable restoring dimerization to the $\operatorname{rad} 9^{\mathrm{MutF}+\mathrm{W}}$ protein. We plan to examine whether the GST protein does indeed restore dimerization to $\operatorname{rad} 9^{\mathrm{MutF}+\mathrm{W}}$ protein by co-immunoprecipitation analysis. We anticipate that dimerization of $\operatorname{rad} 9^{\mathrm{MutF}+\mathrm{W}_{-}}$GST proteins will be restored as it is for $\operatorname{rad} 9 \Delta \mathrm{BRCTs}$ GST proteins. This result, then, would indicate that mutations within the $\operatorname{rad} 9^{\mathrm{MutF}+\mathrm{W}}$ protein cause some dysfunction greater than that of merely abrogating Rad 9 dimerization. 
a)

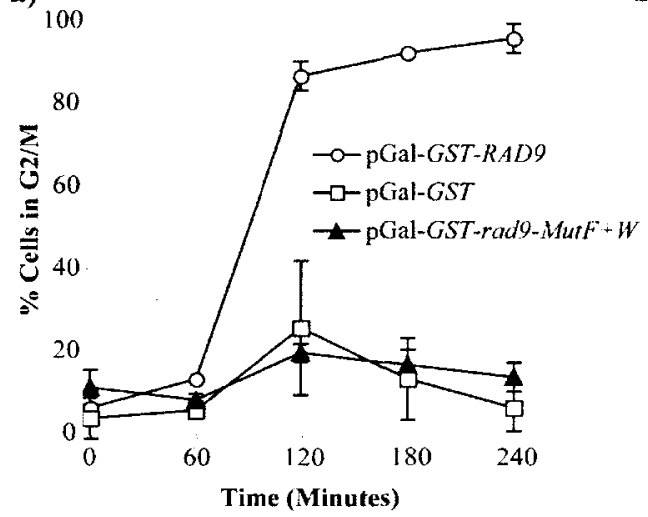

b)

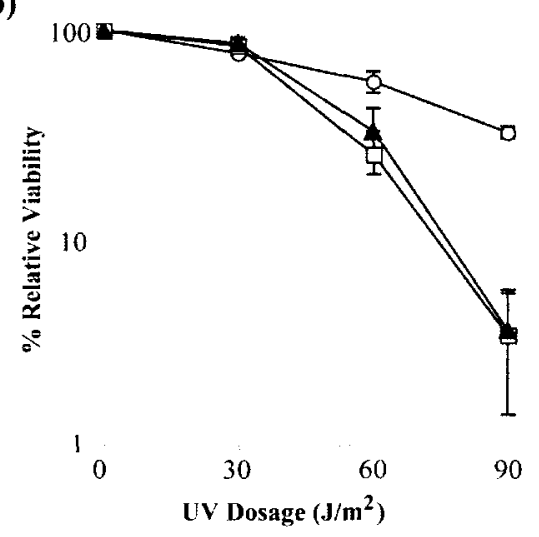

d)
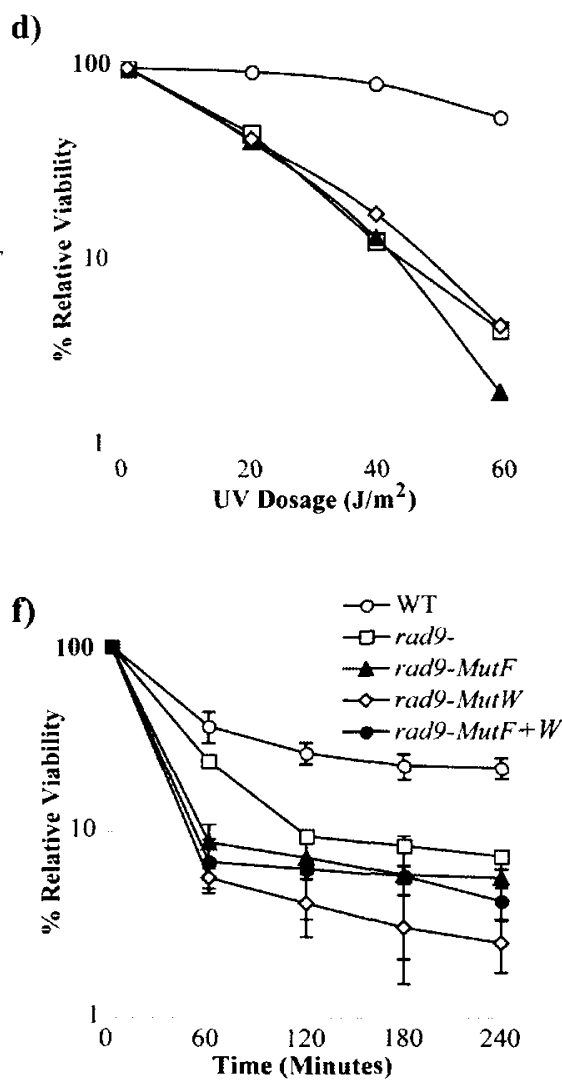

Time (Minutes)

e)
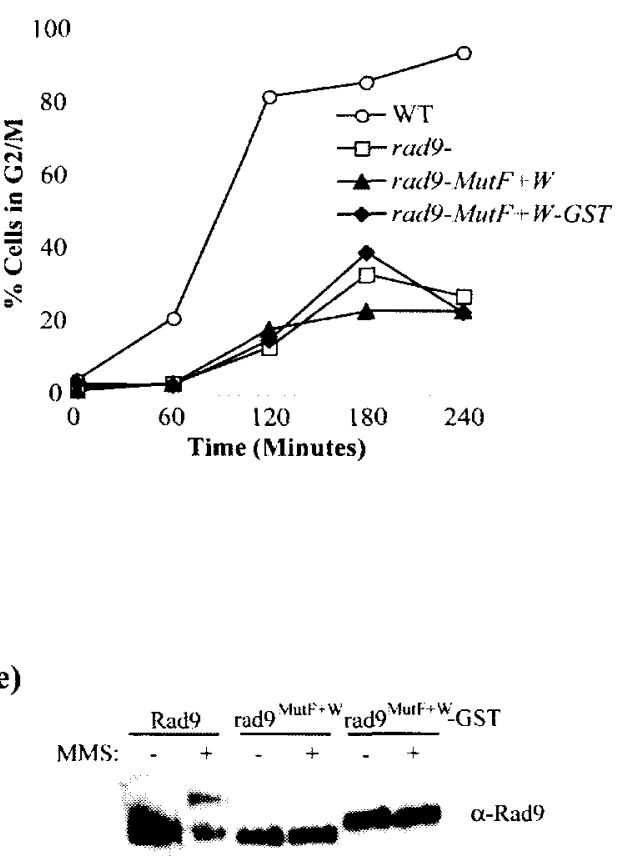

Figure 3-8. Phenotypic and protein characterization of cells containing $\operatorname{rad} 9^{\mathrm{MutF}+\mathrm{W}}$ and rad $9^{\text {MutF+W}}$-GST. (a) Arrest and (b) UV sensitivity of rad 9 cells overexpressing the $\operatorname{rad} 9^{\text {MutF+W }}$ protein. (c) Arrest and $(d)$ UV sensitivity of $r a d 9^{M u t F+W}-G S T$ cells under normal levels of expression. (e) Normal protein expression levels of $\operatorname{rad} 9^{\mathrm{MutF}+\mathrm{W}}$ and $\operatorname{rad} 9^{\mathrm{MutF}+\mathrm{W}}$-GST proteins. (f) MMS sensitivity of $\operatorname{rad} 9^{M u t F}, \operatorname{rad} 9^{M u t W}$, and $\operatorname{rad} 9^{M u t F+W}$. Assays were performed as previously described. 
The $\operatorname{rad} 9^{\mathrm{MutF}}, \operatorname{rad} 9^{\mathrm{MutW}}$, and $\operatorname{rad} 9^{\mathrm{MutF}+\mathrm{W}}$ proteins are all completely defective for $\mathrm{S}$-phase functions.

To gain additional insight into Rad9 S-phase function, we determined the MMS sensitivity of each single BRCT mutant and the double $\operatorname{rad} 9^{M u t F+W}$ mutant. A recent study of the two BRCT domains in $\mathrm{XRCCl}$ involving mutation of the same highly conserved aromatic residues as in the mutated $\operatorname{rad} 9^{M u t F+W}$ allele revealed that mutation of XRCC1 BRCT I rendered cells extremely MMS-sensitive, whereas mutation of BRCT II had little impact on the MMS resistance of cycling Chinese hamster ovary cells (Taylor et al., 2002). Such results suggest that BRCT I in XRCC1 may interact with a specific DNA repair protein to mend MMS-induced damage independent of BRCT II. Following this, we predicted that the single Rad9 BRCT point mutations might also demonstrate a different susceptibility to MMS-induced damage, indicative of different roles for each domain within S-phase. In contrast, we found that each BRCT single mutant was as MMS-sensitive as the double $\operatorname{rad} 9^{M u t F+W}$ mutant (Figure 3-8f), which was identical in sensitivity to that of the $\operatorname{rad} 9^{-}$strain. This suggests that the two domains act in concert to mediate some S-phase function, and that mutation of one is sufficient to render the entire protein completely non-functional. The fact that the two single and the double BRCT point mutants are complete nulls for S-phase function stands in contrast to the phenotype of the $\operatorname{rad} 9 \triangle B R C T s-M Y C$ strain that lacks the BRCT domains altogether yet retains some S-phase function. This result, too, implies a greater protein dysfunction caused by point mutation of the BRCT domains. 
Determining if the null phenotype of $\operatorname{rad} 9^{\mathrm{MutF}+\mathrm{W}}$ can be explained by protein localization defects.

One simple explanation for the strange behavior of the $\operatorname{rad} 9^{\mathrm{MutF}+\mathrm{W}}$ protein is that it is abrogated in its nuclear localization. The protein is expressed in abundances similar to that of wild-type Rad9, yet attempts to restore dimerization do not restore protein function. In contrast, the $\operatorname{rad} 9 \Delta \mathrm{BRCT}$ s-GST protein, which is expressed at abundances much less than that of $\operatorname{Rad} 9$, can fully complement $\operatorname{Rad} 9$ function. The $\operatorname{rad} 9^{\mathrm{MutF}+\mathrm{W}}$ protein demonstrates no ability whatsoever to complement loss of Rad9 activity, whereas even the $\operatorname{rad} 9 \Delta \mathrm{BRCTs}-\mathrm{Mys}$ protein that lacks the BRCT domains altogether demonstrates some UV and MMS-resistance. Even overexpression of the $\operatorname{rad} 9^{\mathrm{MutF}+\mathrm{W}}$ protein has no effect on cell phenotype, while overexpression of rad9 $\triangle B R C T s$ does. All these factors point to a defect that may simply be explained if the $\operatorname{rad} 9^{\mathrm{MutF}+\mathrm{W}}$ protein is not correctly localized to the nucleus. Experiments are currently underway to test this possibility. If the $\operatorname{rad} 9^{\mathrm{MutF}+\mathrm{W}}$ protein does show cellular localization defects, this may have implications for explaining the functional defects of BRCA1 proteins containing similar mutations.

Forcing aberrant Rad9 associations do not affect function.

To elucidate the conditions for Rad9 dimerization, we attempted to produce dominant-negative effects caused by aberrant dimerization using a variety of circumstances. The fact that the BRCT point mutations render overexpressed $\operatorname{rad} 9^{\mathrm{MutF}+\mathrm{W}}$ completely non-functional, whereas cells overexpressing rad9 $\triangle B R C T$ s appear wild-type in response to damage, suggests that $\operatorname{rad} 9^{\mathrm{MutF}+\mathrm{W}}$ is acting in an aberrant manner. To 
possibly account for the difference in phenotype between overexpressed $\operatorname{rad} 9 \Delta \mathrm{BRCTs}$ and normally-expressed $\operatorname{rad} 9^{\mathrm{MutF}+\mathrm{W}}$, we reasoned that the BRCT domains might have a role in addition to that of dimerization by negatively regulating $\operatorname{Rad} 9$ in cis or in trans. By this model, in the case of the $\operatorname{rad} 9 \Delta \mathrm{BRCT}$ s deletion protein, the BRCTs are not present to inhibit protein function by negative regulation. Thus, when overexpressed, $\operatorname{rad} 9 \Delta \mathrm{BRCTs}$ can complement the null rad 9 phenotype by overcoming the need for dimerization by the BRCT domains and by not being negatively regulated by the BRCTs. For the $\operatorname{rad} 9^{\mathrm{MutF}+\mathrm{W}}$ protein, however, the point mutations render the BRCT domains unable to dimerize, as was previously shown, and they yet may retain negative regulation. It is possible that dimerization is required to relieve this negative regulation (although some other protein modification, such as phosphorylation may also be required).

In trying to produce dominant negative effects, we tried several different conditions. We overexpressed the GST protein in the rad9 $\triangle B R C T s$-GST strain background and then induced DNA damage. We also overexpressed the truncated BRCTs and the BRCTs ${ }^{\mathrm{MutF}+\mathrm{W}}$ in the rad9 $B R C T s-G S T$ strain to see if this would produce a dominant negative effect based on a cis negative regulation hypothesis. Moreover, we attempted to force association of rad $9 \Delta B R C T s-G S T$ with the BRCTs by separately expressing GST-BRCTs and GST-BRCTs ${ }^{\mathrm{MutF}+\mathrm{W}}$ proteins in the rad $9 \Delta B R C T s-G S T$ strain background, while then inducing DNA damage to assess the resulting effects. Under all these conditions, we found no dominant negative effect. This may be explained if the affinity of $\operatorname{rad} 9 \triangle \mathrm{BRCTs}-\mathrm{GST}$ dimerization is greater than the affinity for association with 
all the overexpressed fragments tested. A greater affinity of rad9 $\triangle B R C T s-G S T$ association with itself would be predicted if other regions of Rad9 aside from the BRCT domains promoted self-association in addition to the self-associating GST protein. Moreover, this same possibility - that other regions aside from the BRCT domains contribute some low-level affinity for Rad9 self-association - may also account for why we were never able to produce any dominant-negative effects in strains containing endogenous $R A D 9$, assuming that $\operatorname{Rad} 9$ is constitutively dimerized as we predict it to be.

Does unphosphorylatable rad9 still dimerize?

Schwartz et al. (2002) recently identified several [S/T]/Q residues in $\operatorname{Rad} 9$ that undergo Mecl-dependent phosphorylation. Phosphorylation at seven of these sites in combination was shown to be critical for facilitating Rad53 interaction and activation, as the rad9-7xA strain did not activate Rad53 proteins nor could rad9-7xA coimmunoprecipitate Rad53 as wild-type Rad9 did. In contrast, the rad9-7x A strain was completely proficient for activation of another downstream kinase, Chk1, suggesting that phosphorylation of the residues necessary for Rad53 activation has no bearing on Chk 1 activation. Consequently, $\operatorname{rad} 9-7 x A$ cells demonstrated the ability to partially arrest, since activation of Chk1 remained unaffected while Rad53 activation was abrogated.

While the previous study showed that phosphorylation of Rad9 is necessary for Rad53 activation, so, too, is dimerization thought to be important, though the ability of the $\operatorname{rad} 9-7 \mathrm{xA}$ protein to dimerize via its $\mathrm{BRCT}$ domains was not previously assessed in 
this study. Homodimerization is thought to increase the local concentration of Rad9 such that two dimerized, hyperphosphorylated Rad9 molecules can interact with two Rad53 molecules, bringing them in close enough proximity with each other to allow for Rad53 autophosphorylation and, consequently, propagation of the checkpoint cascade (Gilbert et al., 2001). While phosphorylation events needed to activate Rad53 do not affect Chk1 activation, dimerization of Rad9 might be important for Chkl activation, as it is for Rad53.

Indeed, from my work, the $\operatorname{rad} 9 \Delta \mathrm{BRCTs}-\mathrm{Myc}$ protein likely does not dimerize, and in this strain, Chk1 does not undergo phosphorylation in response to damage. In contrast, rad9 $\triangle \mathrm{BRCTs-GST}$ does dimerize, and Chk1 does get activated in this strain. This suggests, then, that dimerization of the Rad9 N-terminus is necessary for Chk 1 phosphorylation in reponse to DNA damage. Consequently, we predict that the rad9-7xA protein should still be able to dimerize, since it can still activate Chkl.

If rad9-7xA can dimerize, this implies that the conditions for Rad9 dimerization are not dependent on its state of hyperphosphorylation by Mecl; the Rad9 phosphorylation event would appear to only affect Rad53 activation, as determined thus far. Moreover, such results would be consistent with the hypothesis that Rad9 is constitutively dimerized regardless of the presence of DNA damage. And if this were true, it would suggest that though Rad9 dimerization is necessary for Chk1 activation, it is not sufficient, or else the checkpoint arrest response would be constitutively partially 
activated. We presume some other Rad9 modification, aside from those that affect Rad53, or some additional protein interaction to be necessary to mediate Chk1 phosphorylation.

Though my data argue against it, if the rad9-7xA protein is unable to dimerize, this would indicate that the residues necessary for Rad53 interaction and activation are also necessary for dimerization. Furthermore, this would suggest that Rad9 dimerization (if it is not constitutive) is dependent on the state of phosphorylation, such that hypophosphorylated Rad9 molecules have a low affinity for dimerization while hyperphosphorylated molecules have a higher affinity for dimerization. This would then also lend insight into the mechanism of Chkl activation, indicating that Chkl activation is not dependent on the state of $\operatorname{Rad} 9$ dimerization.

To test the ability of rad9-7xA to dimerize by co-immunoprecipitation analysis, I created diploid cells containing rad9-7xA-Myc and rad9-7xA-HA proteins. These experiments have yet to be done due to our co-immunoprecipitation problems discussed previously. 


\section{CONCLUSIONS}

\section{Rad9's BRCT domains predominantly function to concentrate Rad9.}

My data indicates that, under normal levels of expression, the BRCT domains within Rad9 mediate homodimer interactions irrespective of DNA damage. Given the phenotypes analyzed thus far, the predominant function of the BRCT domains appears to be to locally concentrate Rad9 by protein homodimerization, since deletion of both domains does not impair the arrest and repair responses of the $\operatorname{rad} 9 \Delta \mathrm{BRCTs}$ protein when it is overexpressed or when dimerization is restored using the GST protein. Additional Rad9 concentration can be achieved by protein stabilization, also mediated by the BRCT domains. Notably, the rad $9 \Delta B R C T s$-GST strain produces cell phenotypes identical to that of wild-type $R A D 9$ for the sensitivity to DNA damage, the kinetics of arrest in response to DNA damage, the kinetics of progression after DNA damage repair, and the extent of adaptation when persistent damage is present. In addition, the cell viabilities between the two strains are identical when cells are treated with either UV or MMS insult or when they are sensitized to MMS damage by addition of the $t o f l$ and $m r c l$ mutations. This indicates that for these functions listed, the BRCT repeats predominantly act to locally concentrate $\operatorname{Rad} 9$ by homodimer formation and protein stabilization.

Furthermore, since the rad $9 \Delta \mathrm{BRCTs}-\mathrm{GST}$ protein is constitutively dimerized and acts identically to $\operatorname{Rad} 9$, this lends support to the idea that $\operatorname{Rad} 9$ is actually dimerized irrespective of DNA damage. Co-immunoprecipitation tests are underway to test this possibility and confirm that the rad $9 \Delta \mathrm{BRCTs-GST}$ proteins are constitutively dimerized. 
Based on the results presented here, we can make certain predictions as to how Rad9 functions. Rad9 homodimerization is thought to be necessary for Rad53 activation. Consequently, homodimerization likely acts to increase the local concentration of Rad 9 such that two dimerized Rad9 molecules can interact with two Rad53 molecules, bringing them in close enough proximity with each other to allow for Rad53 autophosphorylation and, consequently, propagation of the checkpoint cascade. We predict that the state of Rad9 homodimerization is also necessary for Chk1 activation, since the non-dimerizing rad9 $\triangle B R C T s-M Y C$ strain demonstrates no Chk1 phosphorylation in response to DNA damage, whereas the dimerizing rad9 $B R C T S$-GST strain does. We theorize that overexpression of $\operatorname{rad} 9 \triangle \mathrm{BRCT}$ s is sufficient to rescue arrest and repair in a $\operatorname{rad} 9$ null strain by presumably overcoming the need for local concentration by boosting protein concentrations within the cell enough to mediate Rad9 functions. Alternatively, local Rad 9 concentration mediated by BRCT dimerization can be attained by supplanting the BRCTs with another dimerizing domain, such as the GST protein.

Other possible roles mediated by Rad9's BRCT domains.

We hypothesized that the BRCT domains may mediate some other function, such as heterodimer interaction or DNA binding based on reports of other BRCT-containing proteins. If these BRCT functions are operable in $\operatorname{Rad} 9$, we predict them to be redundant with other functions as they have not been identified with the assay conditions described herein. Instead, we find that the BRCT domains may play a role in promoting Rad9 protein stability, since those proteins lacking the BRCTs altogether $-\operatorname{rad} 9 \Delta \mathrm{BRCTs}-\mathrm{Myc}$ 
and $\operatorname{rad} 9 \triangle \mathrm{BRCTs}-\mathrm{GST}$ - are expressed at levels considerably lower under control of the $R A D 9$ promoter than is wild-type Rad9. Curiously, the low protein expression levels do not affect protein function. The rad9 $\triangle B R C T s-G S T$ protein, expressed at one-forth the amount of wild-type Rad9, is still completely proficient for all Rad9 functions.

Stabilizing Rad9 proteins, however, is one means to increase protein concentrations within the cell, though other mechanisms are required to additionally locally concentrate $\operatorname{Rad} 9$ for function.

Another possible role for the Rad9 BRCT domains is indicated by the enigmatic phenotype of the $\operatorname{rad} 9^{M u t F+W}$ strain. The $\operatorname{rad} 9^{\mathrm{MutF}+\mathrm{W}}$ protein is expressed at levels similar to wild-type $\operatorname{Rad} 9$ when under the control of the $R A D 9$ promoter, yet the protein is completely nonfunctional as determined by phenotypic assays. This stands in contrast to the $\operatorname{rad} 9 \Delta \mathrm{BRCTs}-\mathrm{Myc}$ protein that is expressed at greatly reduced levels in the cell compared to wild-type Rad9, yet retains some UV and MMS-resistance function. Furthermore, attempts to restore $\operatorname{rad} 9^{\mathrm{MutF}+\mathrm{W}}$ activity, whether by overexpression or GST fusion, were completely ineffective though such strategies strikingly restored wild-type activity when applied to the rad9 $\triangle \mathrm{BRCTs}$ protein. Explanations that may account for the puzzling rad $9^{\mathrm{MutF}+\mathrm{W}}$ phenotype include protein localization defects or negative regulation defects attributed to mutation of the highly-conserved aromatic residues within each BRCT domain. Additional experiments to test these hypotheses are currently being pursued. 


\section{CHAPTER 4. MATERIALS AND METHODS.}

Strains and media.

Cells were grown in standard media according to standard protocols (see Current Protocols in Molecular Biology). All strains used are shown in Table 4-1 at the end of this chapter.

\section{Cell cycle arrest assay.}

Mid-logarithmic cells were synchronized in $\mathrm{G}_{1}$ by growing at the permissive temperature $\left(23^{\circ} \mathrm{C}\right)$ for 3 hours upon the addition of $\alpha$-factor at a concentration of $2-2.5$ $x 10^{-8}$. Cells were pelleted by centrifugation and washed with $\mathrm{ddH}_{2} \mathrm{O}$ three times to remove $\alpha$-factor. Cells were then resuspended in fresh media to allow cell cycle progression. At this time, cells were shifted to the restrictive temperature $\left(36^{\circ} \mathrm{C}\right)$ to induce $c d c 13-1$ damage. At various times, cells were fixed in $70 \% \mathrm{EtOH}$ and then stained with DAPI (4,6-Diamidino-2-phenylindole) to score by fluorescent microscopy to determine cell cycle position. For each time point, at least 100 total cells were scored and classified as unbudded ( $\mathrm{G}_{1}$-phase), small-budded (S-phase), large-budded ( $\mathrm{G}_{2}$-phase), or large-budded with a divided nucleus (beyond M-phase).

\section{Quantitative UV sensitivity assay.}

Cells were assessed for their ability to survive UV insult, indicative of UV damage repair, based on their viability after UV damage treatment. Mid-logarithmic cells 
were plated to solid media and allowed to dry (to avoid the UV shielding effect of water). Cells were then exposed to various doses of UV using a Stratagene UV Stratalinker 1800 and incubated at $23^{\circ} \mathrm{C}$ overnight to allow cells to recover. The following day, the percentage of viabile cells was determined by microcolony assay using a light microscope, where at least 200 total cells were scored and classified as being either dead ( $<12$ cells per colony) or alive $(>12$ cells per colony). The cell viability values were normalized to the number of viable colonies that grew when subject to no UV exposure.

\section{Quantitative MMS sensitivity assay.}

Quantitative MMS assays were performed in one of two ways. In the first method, mid-logarithmic cells were subcultured into fresh media containing various doses of MMS (typically $0-0.008 \% \mathrm{MMS}$ ) and incubated overnight at $23^{\circ} \mathrm{C}$. The following day, cells were plated to solid media and allowed to grow for 16-30 hours at $23^{\circ} \mathrm{C}$. Viability was assessed by counting the number of macrocolonies visible by the naked eye, normalized to the number of colonies that grew in the presence of no MMS.

In the second quantitative MMS assay, mid-logarithmic cells were incubated with $0.033 \%$ MMS for a certain period of time. Cells were plated to solid media at regular intervals during the time course, grown overnight at $23^{\circ} \mathrm{C}$, and scored the following day by microcolony assay. Again, viability values were normalized to the number of colonies that grew in media containing no MMS. 
Quantitative rapid death assay.

Cells were indirectly tested for the generation of ssDNA at chromosome ends upon $c d c 13-1$ inactivation by this quantitative method. Rapid ssDNA decay is presumed to lead to loss of essential genes and, consequently, cell death. Mid-logarithmic cells were synchronized in $G_{1}$ with $\alpha$-factor at a concentration of $2-2.5 \times 10^{-8}$. Cells were pelleted by centrifugation and washed with $\mathrm{ddH}_{2} \mathrm{O}$ three times to remove $\alpha$-factor. Cells were then resuspended in fresh media to allow cell cycle progression. At this time, cells were shifted to the restrictive temperature $\left(36^{\circ} \mathrm{C}\right)$ to induce $c d c 13-1$ damage. At various times, cells were plated to solid media and incubated at the permissive temperature $\left(23^{\circ} \mathrm{C}\right)$ overnight to allow DNA damage repair and cell recovery. Cells were scored the following day for viability based on microcolony formation. Viability values were normalized to the number of microcolonies that formed when cells were not subject to cdc13-1 damage.

\section{DNA purification for Quantitative Amplification of ssDNA (QAOS).}

To directly determine the quantity of ssDNA present at chromosome ends, DNA was purified from $1 \times 10^{9}$ cells subjected to $c d c 13-1$ damage for various amounts of time. These cells were flash-frozen in liquid nitrogen until all samples had been collected and were ready for DNA isolation. After purifying, this DNA was then probed by the PCRbased Quantitative Amplification of ssDNA (QAOS) method in David Lydall's laboratory. The DNA purification protocol is based on protocols from Wu and Gilbert (1995), Grandin et al. (1997), and the Qiagen Genomic DNA handbook. Unless 
otherwise stated, all steps were performed on ice and solutions were spun in centrifuge rotors that were pre-cooled to $4^{\circ} \mathrm{C}$.

Cells subjected to $c d c 13-1$ damage were thawed on ice, resuspended in $1.0 \mathrm{ml}$ Nuclear Isolation Buffer (17\% glycerol, 10.463 g/L MOPS, 14.72 g/L KOAc, 2 mM $\mathrm{MgCL}_{2}, 0.5 \mathrm{mM}$ spermidine, and $52 \mathrm{mg} / \mathrm{L}$ spermine in $\mathrm{ddH}_{2} \mathrm{O}, \mathrm{pH} 7.2$ ), and transferred to a $2.0 \mathrm{ml}$ Sarstedt screw cap eppendorf tube. Cells were harvested by briefly spinning in a microfuge, aspirating off supernatant, and resuspending in $600 \mu \mathrm{l}$ NIB by vortexing. Glass beads were added to below the meniscus, and cells were lysed at $4^{\circ} \mathrm{C}$ by vortexing until $>90 \%$ breakage was achieved. The lyaste was collected in a $15 \mathrm{ml}$ Falcon tube by puncturing the bottom of the $2 \mathrm{ml}$ Sarstedt tube with a hot 21 -gauge needle and spinning at $2000 \mathrm{rpm}$ for 2 minutes. Beads were washed $2 \mathrm{x}$ with $1 \mathrm{ml} \mathrm{NIB}$ and again spun at 2000 rpm for 2 minutes after each wash to collect the lysate. The lysate was then spun at 6500 $\mathrm{g}$ at $4^{\circ} \mathrm{C}$ for 20 minutes in order to harvest cell nuclei and other cell debris. The supernatant was aspirated and discarded. Isolated nuclei were then dissolved in $2 \mathrm{mls}$ Qiagen buffer $\mathrm{G} 2$ (plus the addition of $200 \mu \mathrm{g} / \mathrm{ml} \mathrm{RNAse} \mathrm{A)} \mathrm{by} \mathrm{incubating} \mathrm{at} 37^{\circ} \mathrm{C}$ for 30 minutes. $50 \mu 120 \mathrm{mg} / \mathrm{ml}$ proteinase $\mathrm{K}$ was added and cells were incubated for another hour at $37^{\circ} \mathrm{C}$. The lysate was cleared by spinning tubes at $6500 \mathrm{~g}$ for 10 minutes at $4^{\circ} \mathrm{C}$. The supernatant, containing the DNA, was then poured into a new round-bottomed $15 \mathrm{ml}$ Falcon tube. The DNA was added to an equilibrated $20 \mathrm{~g}$ Qiagen column and the rest of the steps were completed according to the Qiagen protocol. The purified DNA pellet finally obtained was resuspended in $400 \mu \mathrm{l} \mathrm{TE}$ by rolling overnight at $23^{\circ} \mathrm{C}$. The 
concentration of DNA was determined and then all DNA samples were diluted to a concentration of $2 \mu \mathrm{g} / \mathrm{ml}$. These DNA samples were frozen and shipped on ice to Lydall's laboratory for subsequent PCR analysis using the QAOS method.

Site-Directed mutagenesis of the RAD9 sequence.

Site-directed mutagenesis to create the rad9-T126A and rad9-Y829F alleles was performed using the QuickChange Site-Directed Mutagenesis Kit (Stratagene).

The primer pair used to create the T192A mutation in the $R A D 9$ sequence includes the forward primer 5'- GCG TCA TTA GAA GTT GCA GAG GCG GAT GCC AC -3' and the reverse-complement primer 5'- GTG GCA TCC GCC TCT GCA ACT TCT AAT GAC GC -3'. For the Y829F mutation, the forward primer 5'- CGC TAA CCA AAG ATG AGG ACA TCT TCT ACT TAG ATA TTA GAA TAG GGG -3' and the reversecomplement primer 5'- CCC CTA TTC TAA TAT CTA AGT AGA AGA TGT CCT CAT CTT TGG TTA GCG -3' were used. Only the rad9-Y829F mutation was successfully created and confirmed by DNA sequence analysis (UA DNA Sequencing Service).

Creation of epitope-tagged proteins.

To create epitope-tagged proteins, I utilized the PCR-mediated Pringle tagging method that allowed for modification of the endogenous gene sequences (Longtine et al., 1998). The primer pairs shown in Table 4-2 were used to create proteins with carboxyterminal tags. 
Table 4-2. Pringle primer pairs for $C$-terminally tagging $R A D 9, A S F 1$, and $D D C 1$.

\begin{tabular}{|c|c|c|}
\hline Desired Protein & Primer Direction & Primer Sequence \\
\hline \multirow[t]{2}{*}{ Rad9-Tag } & Forward & $\begin{array}{l}\text { '- TAC GGA CAA TGA TAT ATA } \\
\text { CAA CAC TAT TTC TGA GGT TAG } \\
\text { ACG GAT CCC CGG GTT AAT -3' }\end{array}$ \\
\hline & Reverse & $\begin{array}{l}\text { 5'- TCC CTT TCT ATC AAT TAT GAG } \\
\text { TTT ATA TAT TTT TAT AAT TGA } \\
\text { ATT CGA GCT CGT TTA -3' }\end{array}$ \\
\hline \multirow[t]{2}{*}{$\operatorname{rad} 9 \Delta B R C T s-T a g$} & Forward & $\begin{array}{l}\text { 5'- GAA TCG AGC TCC GAA AAG } \\
\text { AAG GAA ATT GTT AAA AAG GAT } \\
\text { CGG ATC CCC GGG TTA ATT -3' }\end{array}$ \\
\hline & Reverse & $\begin{array}{l}\text { 5'- TCC CTT TCT ATC AAT TAT GAG } \\
\text { TTT ATA TAT TTT TAT AAT TGA } \\
\text { ATT CGA GCT CGT TTA -3' }\end{array}$ \\
\hline \multirow[t]{2}{*}{$\operatorname{rad} 9 \Delta \mathrm{BRCTs}-\mathrm{GST}-\mathrm{Tag}$} & Forward & $\begin{array}{l}\text { 5'- CTG GCA AGC CAC GTT TGG TGG } \\
\text { TGG CGA CCA TCC TCC AAA ACG } \\
\text { GAT CCC CGG GTT AAT TAA -3' }\end{array}$ \\
\hline & Reverse & $\begin{array}{l}\text { 5'- TCC CTT TCT ATC AAT TAT GAG } \\
\text { TTT ATA TAT TTT TAT AAT TGA } \\
\text { ATT CGA GCT CGT TTA -3' }\end{array}$ \\
\hline \multirow[t]{2}{*}{ Asfl-Tag } & Forward & $\begin{array}{l}\text { 5'- TAT TGA ATC CAC TCC AAA GGA } \\
\text { TGC GGC ACG TTC AAC GAA CGG } \\
\text { ATC CCC GGG TTA ATT AA -3' }\end{array}$ \\
\hline & Reverse & $\begin{array}{l}\text { 5'- CTC TCT TGC AGG TAC CAT TAA } \\
\text { TCT TAT AAC CCA TAA ATT CGA } \\
\text { ATT CGA GCT CGT TTA -3' }\end{array}$ \\
\hline \multirow[t]{2}{*}{ Ddc1-Tag } & Forward & $\begin{array}{l}\text { 5'- GGT CTA ACA CAA GTA GAA } \\
\text { AAG CCA AGG GGT ATA TTT GAC } \\
\text { CGG ATC CCC GGG TTA ATT AA -3' }\end{array}$ \\
\hline & Reverse & $\begin{array}{l}\text { 5'- TAA TAT TTA CAC GCC TTT ATA } \\
\text { CTG ATT TTG CAT TAT GGT TGA } \\
\text { ATT CGA GCT CGT TTA }-3\end{array}$ \\
\hline
\end{tabular}




\section{Extraction of total cell protein.}

To extract total cell protein, trichloroacetic acid (TCA) protein precipitation was employed. To ensure equal protein loading by Western blot analysis, equivalent cell densities were subjected to TCA extraction. Typically, $10 \mathrm{ml}$ cell cultures of $\mathrm{OD}_{600}=0.7$ were used. When cells reached this density, they were pelleted and resuspended in $0.5 \mathrm{ml}$ cold $20 \%$ TCA. Cells were then transferred to Eppendorf tubes, pelleted, aspirated, and stored at $-80^{\circ} \mathrm{C}$. To extract protein, cells were thawed on ice. $250 \mu \mathrm{l}$ cold $20 \%$ TCA was added along with glass beads to just below the meniscus. Cells were vortexed at $4^{\circ} \mathrm{C}$ for 20 minutes to lyse cells. Lysate was removed from the beads by puncturing the top and bottom of the Eppendorf tube with an 18 1/2-gauge needle and collecting liquid in 2-ml Eppendorf tubes spun at $1800 \mathrm{rpm}$ for 10 seconds. The beads were then washed with 0.5 $\mathrm{ml}$ cold 5\% TCA and spun again to combine the wash with the lysate. TCA-precipitated cellular proteins were pelleted by spinning at top speed in the cold room for 10 minutes. The supernatant was then aspirated, and the pellets were vortexed to resuspend in $250 \mu \mathrm{l}$ 2x sample loading buffer plus 40-50 $\mu \mathrm{l} 1 \mathrm{M}$ Tris- $\mathrm{Cl}$ (to neutralize residual TCA). Samples were then boiled for 5 minutes, spun briefly to pellet insoluble material, and then loaded onto polyacrylamide gels to separate denatured proteins by electrophoresis.

Immunoprecipitation of cell proteins.

To immunoprecipitate cell proteins, $10 \mathrm{ml}$ cell cultures of $\mathrm{OD}_{600}=0.7$ were typically treated. For low abundance proteins, I sometimes scaled the up the volume of cells treated to either $30 \mathrm{mls} \mathrm{OD}_{600}=0.7$ ( $3 \mathrm{x}$ normal conditions) or $50 \mathrm{mls} \mathrm{OD}_{600}=0.7$ 
(5x normal conditions). Cultures were spun to pellet cells. Cells were then resuspended in water, transferred to Eppendorf tubes, repelleted, and aspirated. (Experiments involving immunoprecipitation of $\operatorname{Rad} 9$ proteins were performed immediately to limit the amount of protein degradation, as freeze-thawing cells promotes Rad 9 degradation. Cells used for immunoprecipitating other proteins, for example Chk 1 , could be frozen at $-80^{\circ} \mathrm{C}$ until future use.) A $3 x$ volume of lysis buffer (e.g., if the cell pellet is $\approx 50 \mu \mathrm{l}$, then 150 $\mu \mathrm{l}$ lysis buffer is added) containing protease inhibitors was then added to the cell pellet (lysis buffer contains $50 \mathrm{mM}$ Tris-acetate $\mathrm{pH} 7.9,150 \mathrm{mM}$ sodium acetate, $10 \mathrm{mM}$ EDTA, $1 \%$ NP-40, 10\% glycerol, $2 \mu \mathrm{g} / \mathrm{ml}$ leupeptin, $2 \mu \mathrm{g} / \mathrm{ml}$ aprotinin, $50 \mu \mathrm{M}$ pepstatin, $2.5 \mathrm{mM}$ PMSF, $2 \mathrm{mM}$ benzamidine, $1 \mathrm{mM}$ DTT, $5 \mathrm{mM}$ sodium orthovanadate, $10 \mathrm{mM} \beta$ glycerophosphate, and $1 \mathrm{mM}$ sodium fluoride). Glass beads were added to just below the meniscus, and cells were vortexed at $4^{\circ} \mathrm{C}$ for 20 minutes. The lysate was collected by piercing the top and bottom of the Eppendorf with an 18 1/2-guage needle and spinning lysate into a $2 \mathrm{ml}$ Eppendorf at $1800 \mathrm{rpm}$ for 10 seconds. The lysis volume was brought up to $500 \mu \mathrm{l}$, and the lysate was spun at top speed at $4^{\circ} \mathrm{C}$ for 10 minutes to pellet insoluble material. The cleared lysate was then added to pre-equilibrated antibodyconjugated sepharose beads. Lysate and beads were allowed to mix with gentle shaking at $4^{\circ} \mathrm{C}$ for 1 hour. The incubations were then briefly spun at $4^{\circ} \mathrm{C}$ to pellet the beads. The lysate was aspirated and beads were washed 3 times with $500 \mu 1$ lysis buffer. After the last wash, all buffer was removed by aspiration and the beads were resuspended in $80 \mu \mathrm{l}$ $2 \mathrm{x}$ sample loading buffer. The resuspended beads were boiled for 5 minutes before loading onto a polyacrylamide gel for separation of denatured proteins by electrophoresis. 
Rad9 and Rad53 proteins were separated on $6 \%$ acrylamide/bis $(37.5: 1)$ gels. The Chkl proteins shown in this work were separated on an $8 \%$ acrylamide/bis $(37.5: 1)$ gel.

Western blot transfer and protein detection.

Denatured proteins separated by electrophoresis in polyacrylamide gels were transferred to nitrocellulose (Bio-Rad Trans-Blot Transfer Medium) using $80 \mathrm{~V}$ power for 45 minutes in a submerged Western transfer apparatus (Bio-Rad). Transfer buffer contained $1 \mathrm{~g}$ SDS, $3.03 \mathrm{~g}$ Tris, $14.42 \mathrm{~g}$ glycine, and $200 \mathrm{ml} \mathrm{100 \%} \mathrm{methanol} \mathrm{brought} \mathrm{up}$ to $1 \mathrm{~L}$ with $\mathrm{ddH}_{2} \mathrm{O}$ :

After protein transfer, the nitrocellulose membrane was incubated in blocking solution ( $5 \%$ non-fat powdered milk resuspended in $1 \mathrm{x}$ TBS $+0.05 \%$ Tween 20 [TBST]) for at least 1 hour or overnight at $4^{\circ} \mathrm{C}$. The membrane was then incubated with shaking with the primary antibody in TBST and 1\% milk block at room temperature for 1 hour. The membrane was washed quickly twice with TBST and then more thoroughly four times for 5 minutes each with shaking. The membrane was incubated with secondary antibody in TBST and 1\% milk block as above, and similar washing conditions were performed after secondary incubation. Proteins were then detected according to standard protocols. 
GFP localization of proteins.

For detection of GFP epitope-tagged proteins, cells were grown to a density of $\mathrm{OD}_{600}=0.3 .200 \mu \mathrm{l}$ of culture was taken and spun to pellet cells. The supernatant was aspirated, and cells were washed with $\mathrm{ddH}_{2} \mathrm{O}$, repelleted, and aspirated three times.

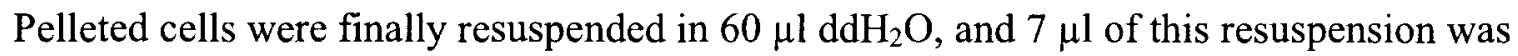
placed on a glass slide for visualization by epifluorescent confocal microscopy. The Molecular and Cellular Biology department Nikon E800 microscope with epifluorescence was used for this purpose.

Nyberg in-gel hybridization procedure.

In an effort to detect the generation of ssDNA at chromosome ends after $c d c 13-1$ damage, I devised an in-gel hybridization protocol somewhat similar to the procedure used by Mundy Wellinger's laboratory (Figure 4-1). Although I never used this protocol to great extent in my analyses, members of other laboratories contacted me about the protocol and are using it regularly.

To detect ssDNA at chromosome ends, a radioactive end-labeled probe must be made. For detection of ssDNA TG tails, the CA oligo (5'- CCC ACC ACA CAC ACC CAC ACC C-3') is ideal. $100 \mathrm{ng}$ of gel-purified CA oligo is labeled in a final volume of $20 \mu \mathrm{l}$ using $1 \mathrm{x}$ polynucleotide kinase (PNK) buffer, $1 \mu \mathrm{l} \mathrm{PNK}$, and $6 \mu \mathrm{l} \gamma$-ATP. The reaction is incubated at $37^{\circ} \mathrm{C}$ for 45 minutes, and the kinase is then inactivated by heating at $70^{\circ} \mathrm{C}$ for 10 minutes. To precipitate and purify the probe, $80 \mu 1 \mathrm{ddH}_{2} \mathrm{O}, 125 \mu 17.5 \mathrm{M}$ $\mathrm{NH}_{4} \mathrm{OAc}$, and $530 \mu \mathrm{l}$ cold $\mathrm{EtOH}$ is added, and the mixture is kept at $-20^{\circ} \mathrm{C}$ for at least an 
hour. The probe is then spun at maximum speed for 45 minutes at $4^{\circ} \mathrm{C}$, washed once with cold $70 \%$ EtOH, spun again for 15 more minutes, air-dried, and then resuspended in 30 $50 \mu \mathrm{l} \mathrm{TE}$. One $\mu \mathrm{l}$ of this resuspension should yield on the order of $1-2 \times 10^{5}$ counts per minute. The probe should be resuspended in the appropriate volume of TE so that $1 \mu \mathrm{l}$ contains enough probe to yield 50,000 counts per minute.

The DNA to be tested for ssDNA presence should be digested with a restriction enzyme. Typically, I digested 40-50 $\mu \mathrm{g}$ genomic DNA with XhoI. When the digest is complete, the restriction enzyme is heat-inactivated. At this point, $50,000 \mathrm{cpm}$ of the end-labeled $\mathrm{CA}$ oligo is added to each digest reaction tube and heated at $37^{\circ} \mathrm{C}$ for 10 minutes. Tubes are then placed on ice for 30 minutes to allow the probe to anneal to ssDNA tails. DNA digests and annealed probe are then separated by agarose gel electrophoresis. An unlabeled DNA ladder and 50,000 cpm of a radioactively-labeled ladder should also be run as standards for comparison. The gel is run overnight at very low voltage, typically $25-35$ volts. When the gel has completed running, stain with ethidium bromide, and photograph. The gel is then dried on a gel dryer (I used a Bio-Rad 583 model) at room temperature using no heat. The gel is placed with the open wells facing down on Whatman paper and covered with saran wrap. The gel should be dried for approximately $20-40$ minutes until the gel is very thin (approximately $1 \mathrm{~mm}$ ) and even. The dried gel should be wrapped in saran wrap and placed on a phosphorimager screen or film for development. The image should be allowed to develop at least overnight, as the signal strength is typically not that strong. 


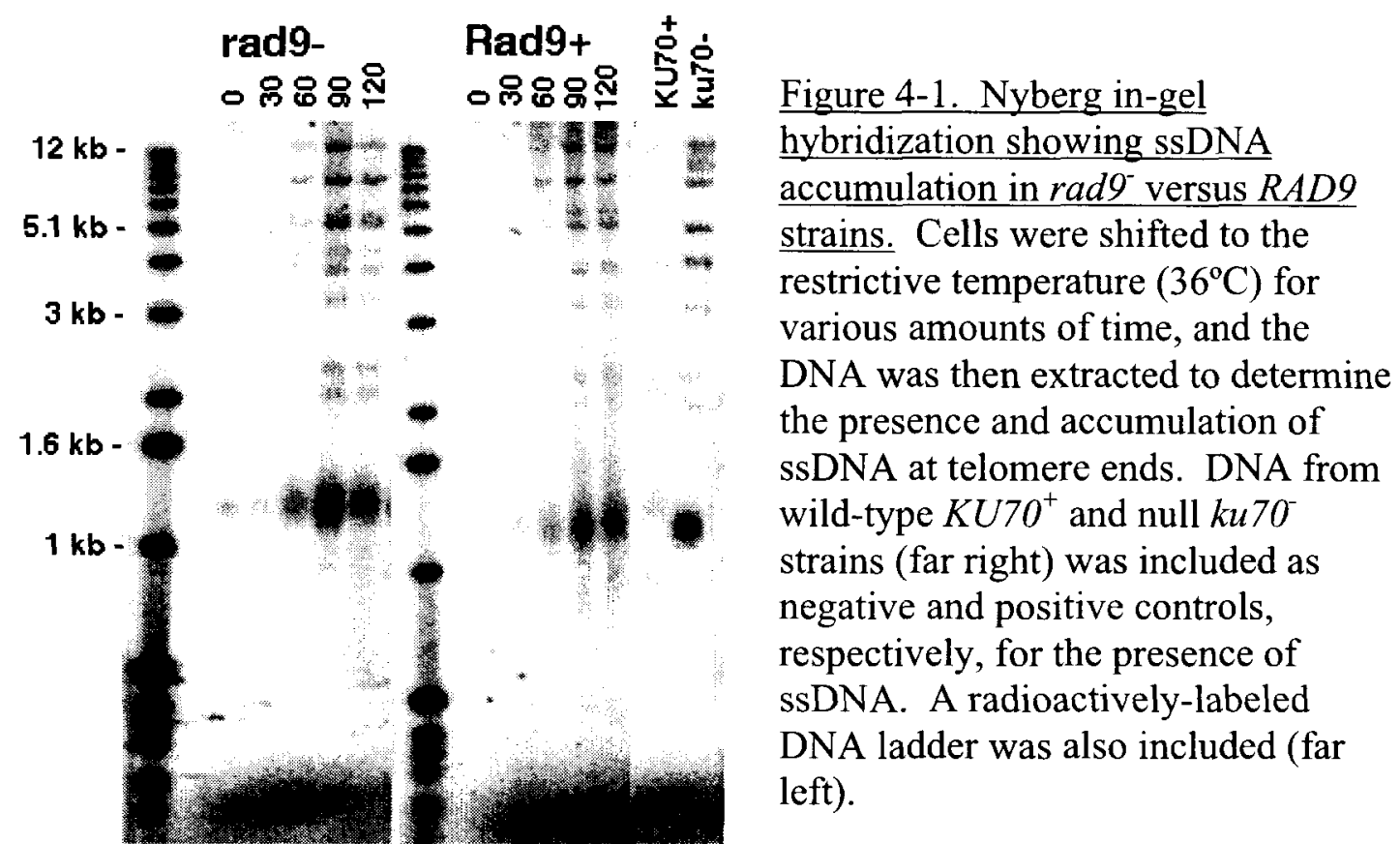


Table 4-1. Strains used in this study. All W303 strains are isogenic to ade 2 can 1 his 3 leu2 trp1 ura3 rad5. The A364a background is not known. Only deviations from these genotypes are listed.

\begin{tabular}{|c|c|c|}
\hline Strain & Genotype & $\begin{array}{l}\text { Back- } \\
\text { ground }\end{array}$ \\
\hline KNY1 & $\begin{array}{l}\text { MATa barl cdc13-1 cdc15 rad9::HIS3 ura3::URA3 } \\
\text { rad17::LEU2 }\end{array}$ & W303 \\
\hline KNY3 & $\begin{array}{l}\text { MATa barl cdc13-1 cdc15 rad9::HIS3 ura3::RAD9-URA3 } \\
\text { rad17::LEU2 }\end{array}$ & W303 \\
\hline KNY5 & $\begin{array}{l}\text { MATa barl cdc13-1 cdc15 rad9::HIS3 ura3::rad9-Y829F- } \\
\text { URA3 rad17::LEU2 }\end{array}$ & W303 \\
\hline KNY7 & $\begin{array}{l}\text { MATa barl cdc13-1 cdc15 mec1-1 smll rad9::HIS3 } \\
\text { ura3::URA3 }\end{array}$ & W303 \\
\hline KNY9 & $\begin{array}{l}\text { MATa barl cdc13-1 cdc15 mec1-1 sml1 rad9::HIS3 } \\
\text { ura3::RAD9-URA3 }\end{array}$ & W303 \\
\hline KNY11 & $\begin{array}{l}\text { MATa barl cdc13-1 cdc15 mecl-1 sml1 rad9::HIS3 } \\
\text { ura3::rad9-Y829F-URA3 }\end{array}$ & W303 \\
\hline KNY17 & MATa barl cdc13-1 rad9::HIS3 ura3::rad9-11-URA3 & W303 \\
\hline KNY19 & MATa barl cdc13-1 rad9::HIS3 ura3::rad9 $\triangle B R C T s-U R A 3$ & W303 \\
\hline KNY21 & MATa barl cdc13-1 rad9::HIS3 + pELP41 (GAL-GST-URA3) & W303 \\
\hline KNY23 & $\begin{array}{l}\text { MATa barl cdc13-1 rad9::HIS3 + pELP40 (GAL-GST::RAD9- } \\
\text { URA3) }\end{array}$ & W303 \\
\hline $\mathrm{KNY} 25$ & 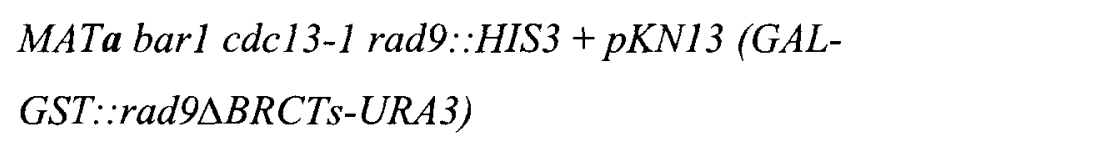 & W303 \\
\hline KNY27 & $\begin{array}{l}\text { MATa barl cdc13-1 rad9::HIS3 + pKN12 (GAL-GST::BRCTs- } \\
\text { URA3) }\end{array}$ & W303 \\
\hline KNY30 & MATa $\operatorname{rad} 9^{M u t F}-H I S 3$ (source: Noel Lowndes) & W303 \\
\hline KNY31 & MATa $\operatorname{rad} 9^{M u t W}-H I S 3$ (source: Noel Lowndes) & W303 \\
\hline KNY32 & MATa $\operatorname{rad} 9^{M u t F+W}-H I S 3$ (source: Noel Lowndes) & W303 \\
\hline
\end{tabular}


KNY36 MATa barl cdc13-1 RAD9::MYC-HIS3 W303

KNY38 MAT $\alpha$ barl cdc13-1 RAD9::HA-TRP1 W303

KNY40 MATa barl cdc13-1 rad9ABRCTs::MYC-TRP1 W303

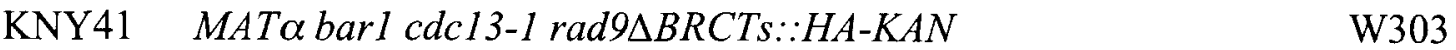

KNY42 MATa barl cdc13-1 rad9ABRCTs::GST-TRPI W303

KNY43 MATa barl cdc13-1 RAD9::MYC-KAN W303

KNY45 MATa barl cdc13-1 rad9ABRCTs::GST-TRP1 W303

KNY46 MATa barl cdc13-1 rad9ABRCTs::GST::HA-KAN W303

KNY48 MAT $\alpha$ barl cdc13-1 rad9ABRCTs::GST::MYC-HIS3 W303

KNY50 MATa barl cdc13-1 cdc15 chk1::HIS3 dun1::TRP1 W303

KNY52 MATa/MAT $\alpha$ bar1/barl cdc13-1/cdc13-1 rad9 $\triangle B R C T s:: M Y C-\quad$ W303 TRP1/rad9 $\triangle B R C T s:: H A-K A N$

KNY54 MATa/MAT $\alpha$ barl/barl cdc13-1/cdc13-1

W303 rad9ABRCTs::GST::HA-KAN/rad9DBRCTs::GST::MYC-HIS3

KNY56 MATa tof $1:: N E O$

W303

KNY58 MATa barl cdc13-1 cdc15 chk1::HIS3 W303

KNY59 MATa barl cdc13-1 cdc15 chk1::HIS3 rad17::LEU2 W303

KNY61 MATa barl cdc13-1 cdc15 dun1::HIS3 rad17::LEU2 W303

KNY63 MATa barl cdc13-1 cdc15 chk1::HIS3 rad9::LEU2 W303

KNY64 MATa barl cdc13-1 cdc15 dun1::HIS3 rad9::LEU2 W303

KNY65 MATa bar1 cdc13-1 cdc15 dun1::HIS3 ura3::rad9-Y829F- $\quad$ W303 URA3

KNY67 MATa barl cdc13-1 rad9::HIS3 tofl::NEO W303

KNY69 MATa barl cdc13-1 tofl::NEO W303

KNY71 MATa bar1 cdc13-1 cdc15 rad9::HIS3 + pGAL-URA3 W303

KNY73 MATa barl cdc13-1 cdc15 rad9::HIS3 + pGAL-RAD9-URA3 W303

KNY75 MATa barl cdc13-1 cdc15 rad9::HIS3 + pKN19 (GAL- W303 $\operatorname{rad} 9 \Delta B R C T s-U R A 3)$

KNY77 MATa barl cdc13-1+pEL41 (GAL-GST-URA3) W303 
KNY79 MATa barl cdc13-1+pEL40 (GAL-GST:RAD9-URA3) W303

KNY81 MATa bar1 cdc13-1 + pKN13 (GAL-GST::rad9ABRCTs-URA3) W303

KNY83 MATa barl cdc13-1 + pKN12 (GAL-GST::BRCTs-URA3) W303

KNY85 MATa barl cdc13-1 + pKN20 (GAL-GST::rad9 $\left.9^{M u t F+W}-U R A 3\right) \quad$ W303

KNY87 MATa barl cdc13-1 RAD9::GST-HIS3 W303

KNY88 MATa (source: Andrew Emili) A364a

KNY89 MATa asfl : KAN (source: Andrew Emili) A364a

KNY90 MAT $\alpha c d c 13-1$ (source: Andrew Emili) A364a

KNY91 MATa $c d c 13-1$ asf1::KAN (source: Andrew Emili) A364a

KNY92 MATa cdc13-1 asf1::KAN rad17::LEU2 A364a

KNY94 MATa barl cdc13-1 asf1 : KAN rad9::HIS3 $\quad$ W303

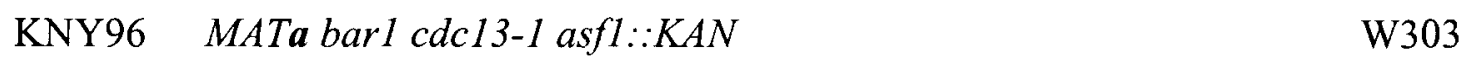

KNY98 MATa barl cdc13-1 asf1::KAN rad9::HIS3 rad17::LEU2 W303

KNY100 MATa barl cdc13-1 RAD9::MYC-HIS3 RAD53::FLAG-LEU2 W303

KNY101 MATa barl cdc13-1 rad9ABRCTs::MYC-HIS3 RAD53::FLAG- W303 LEU2

KNY102 MATa barl cdc13-1 rad9ABRCTs::GST-TRP RAD53::FLAG- W303 LEU2

KNY103 MATa barl cdc13-1 rad9 $9^{\mathrm{MutF}+W_{-H I S 3}}$ W303

KNY104 MATa barl cdc13-1 rad9 ${ }^{M u t F+W}:: G S T-T R P 1 \quad$ W303

KNY106 MATa barl cdc13-1 RAD9::GST-TRPI W303

KNY108 MATa barl cdc13-1 rad9::HIS3 RAD53::FLAG-LEU2 W303

KNY109 MATa barl cdc13-1 RAD53::FLAG-LEU2 W303

KNY111 MATa barl cdc13-1 tofl::NEO RAD9::MYC-HIS3 W303

KNY112 MATa barl cdc13-1 tofl :NEO rad9DBRCTs::MYC-TRP1 W303

KNY113 MATa barl cdc13-1 tofl :NEO rad9ABRCTs::GST-TRP1 W303

KNY114 MATa barl cdc13-1 mrcl::NEO W303

KNY115 MATa barl cdc13-1 mrc1::NEO RAD9::MYC-HIS3 W303

KNY116 MATa barl cdc13-1 mrc1::NEO rad9ABRCTs::MYC-TRPI W303 
KNY117 MATa barl cdc13-1 mrc1::NEO rad9ABRCTs::GST-TRP1 W303

KNY118 MATa/Matabar1/barl cdc13-1/cdc13-1 RAD9::MYC-

W303 HIS3/RAD9::HA-KAN

KNY119 MATa barl cdc13-1 rad9 $: \because H I S 3+p C H K 1: \because M Y C-U R A 3$

W303

KNY120 MATa barl cdc13-1 + pCHK1::MYC-URA3

W303

KNY121 MATa barl cdc13-1 RAD9::MYC-HIS3 + pCHK1::MYC-URA3

W303

KNY122 MATa barl cdc13-1 rad9 $B R C T s:: M Y C-T R P 1+$

W303 pCHK1::MYC-URA3

KNY123 MATa barl cdc13-1 rad9 $\triangle B R C T s:: M Y C-T R P 1+$

W303 pCHK1::MYC-URA3 + prad9 $B R C T s:: M Y C-L E U 2(2 \mu)$

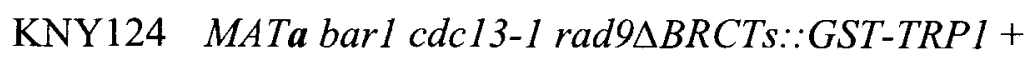

W303 pCHK1::MYC-URA3

KNY125 MATa/Mata barl/bar1 cdc13-1/cdc13-1 RAD9::MYC-

W303 HIS3/RAD9::HA-KAN+pCDC13

KNY126 MATa/Matabarl/barl cdc13-1/cdc13-1

W303

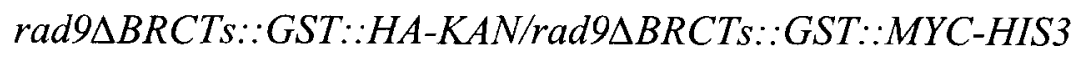
$+p C D C 13$

KNY127 MAT $\alpha$ barl cdc13-1 rad9 $9^{M u t F+W}-H I S 3$

W303

KNY129 MATa barl cdc13-1 rad9 $9^{M u t F+W}:$ GST-TRP1

W303

KNY130 MAT $\alpha$ barl cdc13-1 rad9 $9^{M u t F+W}:: G S T-T R P 1$

W303

KNY131 MATa barl cdc13-1 rad9 $9^{M u t F+W}:: G S T:: H A-K A N$

W303

KNY134 MAT $\alpha$ barl cdc13-1 rad9 $9^{M u t F+W}: \because G S T:: M Y C-H I S 3$ W303

KNY135 MATa/MATa barlbarl cdc13-1cdc13-1 rad9 $9^{M u t F+W}:: G S T:: H A-$ W303 $K A N /$ rad $9^{M u t F+W}:: G S T:: M Y C-H I S 3$

KNY138 MATa barl cdc13-1 rad9ABRCTs::MYC-TRP1 RAD53::FLAG- W303 LEU2

KNY139 MATa barl cdc13-1 rad9 $B R C T s:: M Y C-T R P 1 R A D 53:: F L A G-$ W303 $L E U 2+p 2 \mu \operatorname{rad} 9 \Delta B R C T s:: M Y C-U R A 3$

KNY140 MATa barl cdc13-1 rad9-7xA::HA-URA3 
KNY142 MAT $\alpha$ barl cdc13-1 rad9-7xA::HA-URA3 W303

KNY144 MATabarl cdc13-1 rad9-7xA::MYC-TRP1 W303

KNY145 MATa/MAT $\alpha$ bar1/barl cdc13-1/cdc13-1 rad9-7xA :HA- W303 URA3/rad9-7xA::MYC-TRP1

KNY147 MATa barl cdc13-1 rad9 $\triangle B R C T s:: M Y C-T R P 1+p 2 \mu \quad$ W303 rad9 $\triangle B R C T s:: M Y C-U R A 3$

KNY149 MATa barl cdc13-1 rad9ABRCTs::GST-TRPI RAD53::FLAG- W303 $L E U 2$

KNY151 MATa barl cdc13-1 ASF1::GFP-TRP1 W303

KNY153 MATa barl cdc13-1 ASF1::GFP-TRP1 rad9::HIS3 W303

KNY155 MATa barl cdc13-1 DDC1::GFP-TRP1 W303

KNY157 MATa barl cdc13-1 DDC1::GFP-TRP1 rad9::HIS3 W303 


\section{CHAPTER 5. REFERENCES}

Abbott, D.W., Thompson, M.E., Robinson-Benion, C., Tomlinson, G., Jensen, R.A. and Holt, J.T. (1999) BRCA1 expression restores radiation resistance in BRCA1-defective cancer cells through enhancement of transcription-coupled DNA repairJ Biol Chem, 274, 18808-18812.

Aboussekhra, A. and Thoma, F. (1998) Nucleotide excision repair and photolyase preferentially repair the nontranscribed strand of RNA polymerase III-transcribed genes in Saccharomyces cerevisiae. Genes Dev, 12, 411-421.

Abraham, R.T. (2001) Cell cycle checkpoint signaling through the ATM and ATR kinases. Genes Dev, 15, 2177-2196.

Ahmed, S. and Hodgkin, J. (2000) MRT-2 checkpoint protein is required for germline immortality and telomere replication in C. elegans. Nature, 403, 159-164.

al-Khodairy, F., Fotou, E., Sheldrick, K.S., Griffiths, D.J., Lehmann, A.R. and Carr, A.M. (1994) Identification and characterization of new elements involved in checkpoint and feedback controls in fission yeast. Mol Biol Cell, 5, 147-160.

Aladjem, M.I., Spike, B.T., Rodewald, L.W., Hope, T.J., Klemm, M., Jaenisch, R. and Wahl, G.M. (1998) ES cells do not activate p53-dependent stress responses and undergo p53-independent apoptosis in response to DNA damage. Curr Biol, 8, 145-155.

Alcasabas, A.A., Osborn, A.J., Bachant, J., Hu, F., Werler, P.J., Bousset, K., Furuya, K., Diffley, J.F., Carr, A.M. and Elledge, S.J. (2001) Mrcl transduces signals of DNA replication stress to activate Rad53. Nat Cell Biol, 3, 958-965.

Amon, A. (2001) Together until separin do us part. Nat Cell Biol, 3, E12-14.

Ando, T., Kawabe, T., Ohara, H., Ducommun, B., Itoh, M. and Okamoto, T. (2001) Involvement of the interaction between $\mathrm{p} 21$ and proliferating cell nuclear antigen for the maintenance of G2/M arrest after DNA damage. J Biol Chem, 276, 42971-42977.

Araki, H., Leem, S.H., Phongdara, A. and Sugino, A. (1995) Dpb11, which interacts with DNA polymerase II(epsilon) in Saccharomyces cerevisiae, has a dual role in S-phase progression and at a cell cycle checkpoint. Proc Natl Acad Sci U S A, 92, 11791-11795.

Baber-Furnari, B.A., Rhind, N., Boddy, M.N., Shanahan, P., Lopez-Girona, A. and Russell, P. (2000) Regulation of mitotic inhibitor Mik1 helps to enforce the DNA damage checkpoint. Mol Biol Cell, 11, 1-11.

Banin, S., Moyal, L., Shieh, S., Taya, Y., Anderson, C.W., Chessa, L., Smorodinsky, 
N.I., Prives, C., Reiss, Y., Shiloh, Y. and Ziv, Y. (1998) Enhanced phosphorylation of p53 by ATM in response to DNA damage. Science, 281, 1674-1677.

Bargonetti, J. and Manfredi, J.J. (2002) Multiple roles of the tumor suppressor p53. Curr Opin Oncol, 14, 86-91.

Bartek, J. and Lukas, J. (2001) Mammalian G1- and S-phase checkpoints in response to DNA damage. Curr Opin Cell Biol, 13, 738-747.

Bartek, J. and Lukas, J. (2001) Pathways governing G1/S transition and their response to DNA damage. FEBS Lett, 490, 117-122.

Bashkirov, V.I., King, J.S., Bashkirova, E.V., Schmuckli-Maurer, J. and Heyer, W.D. (2000) DNA repair protein Rad55 is a terminal substrate of the DNA damage checkpoints. Mol Cell Biol, 20, 4393-4404.

Bell, D.W., Varley, J.M., Szydlo, T.E., Kang, D.H., Wahrer, D.C., Shannon, K.E., Lubratovich, M., Verselis, S.J., Isselbacher, K.J., Fraumeni, J.F., Birch, J.M., Li, F.P., Garber, J.E. and Haber, D.A. (1999) Heterozygous germ line hCHK2 mutations in LiFraumeni syndrome. Science, 286, 2528-2531.

Bhattacharyya, A., Ear, U.S., Koller, B.H., Weichselbaum, R.R. and Bishop, D.K. (2000) The breast cancer susceptibility gene BRCA1 is required for subnuclear assembly of Rad51 and survival following treatment with the DNA cross-linking agent cisplatin. $J$ Biol Chem, 275, 23899-23903.

Boddy, M.N., Lopez-Girona, A., Shanahan, P., Interthal, H., Heyer, W.D. and Russell, P. (2000) Damage tolerance protein Mus81 associates with the FHA1 domain of checkpoint kinase Cds1. Mol Cell Biol, 20, 8758-8766.

Booth, C., Griffith, E., Brady, G. and Lydall, D. (2001) Quantitative amplification of single-stranded DNA (QAOS) demonstrates that cdc13-1 mutants generate ssDNA in a telomere to centromere direction. Nucleic Acids Res, 29, 4414-4422.

Bork, P., Hofmann, K., Bucher, P., Neuwald, A.F., Altschul, S.F. and Koonin, E.V. (1997) A superfamily of conserved domains in DNA damage-responsive cell cycle checkpoint proteins. Faseb $J, \mathbf{1 1}, 68-76$.

Bressan, D.A., Baxter, B.K. and Petrini, J.H. (1999) The Mre11-Rad50-Xrs2 protein complex facilitates homologous recombination-based double-strand break repair in Saccharomyces cerevisiae. Mol Cell Biol, 19, 7681-7687.

Brown, E.J. and Baltimore, D. (2000) ATR disruption leads to chromosomal fragmentation and early embryonic lethality. Genes Dev, 14, 397-402. 
Brush, G.S., Morrow, D.M., Hieter, P. and Kelly, T.J. (1996) The ATM homologue $\mathrm{MECl}$ is required for phosphorylation of replication protein A in yeast. Proc Natl Acad Sci US A, 93, 15075-15080.

Brush, G.S. and Kelly, T.J. (2000) Phosphorylation of the replication protein A large subunit in the Saccharomyces cerevisiae checkpoint response. Nucleic Acids Res, 28, 3725-3732.

Bulavin, D.V., Higashimoto, Y., Popoff, I.J., Gaarde, W.A., Basrur, V., Potapova, O., Appella, E. and Fornace, A.J., Jr. (2001) Initiation of a G2/M checkpoint after ultraviolet radiation requires p38 kinase. Nature, 411, 102-107.

Bulavin, D.V., Amundson, S.A. and Fornace, A.J. (2002) p38 and Chkl kinases: different conductors for the G(2)/M checkpoint symphony. Curr Opin Genet Dev, 12, 92-97.

Burtelow, M.A., Roos-Mattjus, P.M., Rauen, M., Babendure, J.R. and Karnitz, L.M. (2001) Reconstitution and molecular analysis of the hRad9-hHus1-hRad1 (9-1-1) DNA damage responsive checkpoint complex. J Biol Chem, 276, 25903-25909.

Buscemi, G., Savio, C., Zannini, L., Micciche, F., Masnada, D., Nakanishi, M., Tauchi, H., Komatsu, K., Mizutani, S., Khanna, K., Chen, P., Concannon, P., Chessa, L. and Delia, D. (2001) Chk2 activation dependence on Nbs1 after DNA damage. Mol Cell Biol, 21, 5214-5222.

Caldecott, K.W., Tucker, J.D., Stanker, L.H. and Thompson, L.H. (1995)

Characterization of the XRCC1-DNA ligase III complex in vitro and its absence from mutant hamster cells. Nucleic Acids Res, 23, 4836-4843.

Callebaut, I. and Mornon, J.P. (1997) From BRCA1 to RAP1: a widespread BRCT module closely associated with DNA repair. FEBS Lett, 400, 25-30.

Canman, C.E., Lim, D.S., Cimprich, K.A., Taya, Y., Tamai, K., Sakaguchi, K., Appella, E., Kastan, M.B. and Siliciano, J.D. (1998) Activation of the ATM kinase by ionizing radiation and phosphorylation of p53. Science, 281, 1677-1679.

Carney, J.P., Maser, R.S., Olivares, H., Davis, E.M., Le Beau, M., Yates, J.R., 3rd, Hays, L., Morgan, W.F. and Petrini, J.H. (1998) The hMre11/hRad50 protein complex and Nijmegen breakage syndrome: linkage of double-strand break repair to the cellular DNA damage response. Cell, 93, 477-486.

Carr, A.M. (2000) Cell cycle. Piecing together the p53 puzzle. Science, 287, 1765-1766.

Caspari, T., Dahlen, M., Kanter-Smoler, G., Lindsay, H.D., Hofmann, K., Papadimitriou, K., Sunnerhagen, P. and Carr, A.M. (2000) Characterization of Schizosaccharomyces 
pombe Hus 1: a PCNA-related protein that associates with Rad1 and Rad9. Mol Cell Biol, 20, 1254-1262.

Chabes, A., Domkin, V. and Thelander, L. (1999) Yeast Smll, a protein inhibitor of ribonucleotide reductase. J Biol Chem, 274, 36679-36683.

Chakraverty, R.K., Kearsey, J.M., Oakley, T.J., Grenon, M., de La Torre Ruiz, M.A., Lowndes, N.F. and Hickson, I.D. (2001) Topoisomerase III acts upstream of Rad53p in the S-phase DNA damage checkpoint. Mol Cell Biol, 21, 7150-7162.

Chamankhah, M. and Xiao, W. (1999) Formation of the yeast Mre11-Rad50-Xrs2 complex is correlated with DNA repair and telomere maintenance. Nucleic Acids Res, 27, 2072-2079.

Chan, T.A., Hermeking, H., Lengauer, C., Kinzler, K.W. and Vogelstein, B. (1999) 14-33 Sigma is required to prevent mitotic catastrophe after DNA damage. Nature, 401, 616620 .

Chehab, N.H., Malikzay, A., Appel, M. and Halazonetis, T.D. (2000) Chk2/hCds 1 functions as a DNA damage checkpoint in $\mathrm{G}(1)$ by stabilizing p53. Genes Dev, 14, 278288.

Cheng, L., Hunke, L. and Hardy, C.F. (1998) Cell cycle regulation of the Saccharomyces cerevisiae polo-like kinase cdc5p. Mol Cell Biol, 18, 7360-7370.

Clerici, M., Paciotti, V., Baldo, V., Romano, M., Lucchini, G. and Longhese, M.P. (2001) Hyperactivation of the yeast DNA damage checkpoint by TEL1 and DDC2 overexpression. Embo J, 20, 6485-6498.

Cohen-Fix, O. and Koshland, D. (1997) The anaphase inhibitor of Saccharomyces cerevisiae Pds lp is a target of the DNA damage checkpoint pathway. Proc Natl Acad Sci $U S A, 94,14361-14366$.

Corda, Y., Schramke, V., Longhese, M.P., Smokvina, T., Paciotti, V., Brevet, V., Gilson, E. and Geli, V. (1999) Interaction between Setlp and checkpoint protein Mec3p in DNA repair and telomere functions. Nat Genet, 21, 204-208.

Cortez, D., Wang, Y., Qin, J. and Elledge, S.J. (1999) Requirement of ATM-dependent phosphorylation of brcal in the DNA damage response to double-strand breaks. Science, 286, $1162-1166$.

Cortez, D., Guntuku, S., Qin, J. and Elledge, S.J. (2001) ATR and ATRIP: partners in checkpoint signaling. Science, 294, 1713-1716. 
Costanzo, V., Robertson, K., Ying, C.Y., Kim, E., Avvedimento, E., Gottesman, M., Grieco, D. and Gautier, J. (2000) Reconstitution of an ATM-dependent checkpoint that inhibits chromosomal DNA replication following DNA damage. Mol Cell, 6, 649-659.

Cox, M.M., Goodman, M.F., Kreuzer, K.N., Sherratt, D.J., Sandler, S.J. and Marians, K.J. (2000) The importance of repairing stalled replication forks. Nature, 404, 37-41.

Craven, R.J. and Petes, T.D. (2000) Involvement of the checkpoint protein Meclp in silencing of gene expression at telomeres in Saccharomyces cerevisiae. Mol Cell Biol, 20, 2378-2384.

Crawford, D.F. and Piwnica-Worms, H. (2001) The G(2) DNA damage checkpoint delays expression of genes encoding mitotic regulators. $J$ Biol Chem, 276, 37166-37177.

D'Amours, D. and Jackson, S.P. (2002) The mre11 complex: at the crossroads of dna repair and checkpoint signalling. Nat Rev Mol Cell Biol, 3, 317-327.

Davey, S., Han, C.S., Ramer, S.A., Klassen, J.C., Jacobson, A., Eisenberger, A., Hopkins, K.M., Lieberman, H.B. and Freyer, G.A. (1998) Fission yeast rad12+ regulates cell cycle checkpoint control and is homologous to the Bloom's syndrome disease gene. Mol Cell Biol, 18, 2721-2728.

de Klein, A., Muijtjens, M., van Os, R., Verhoeven, Y., Smit, B., Carr, A.M., Lehmann, A.R. and Hoeijmakers, J.H. (2000) Targeted disruption of the cell-cycle checkpoint gene ATR leads to early embryonic lethality in mice. Curr Biol, 10, 479-482.

de la Torre Ruiz, M.A. and Lowndes, N.F. (2000) DUN1 defines one branch downstream of RAD53 for transcription and DNA damage repair in Saccharomyces cerevisiae. FEBS Lett, 485, 205-206.

Desany, B.A., Alcasabas, A.A., Bachant, J.B. and Elledge, S.J. (1998) Recovery from DNA replicational stress is the essential function of the S-phase checkpoint pathway. Genes Dev, 12, 2956-2970.

Di Leonardo, A., Linke, S.P., Clarkin, K. and Wahl, G.M. (1994) DNA damage triggers a prolonged p53-dependent $\mathrm{G} 1$ arrest and long-term induction of Cipl in normal human fibroblasts. Genes Dev, 8, 2540-2551.

Dolganov, G.M., Maser, R.S., Novikov, A., Tosto, L., Chong, S., Bressan, D.A. and Petrini, J.H. (1996) Human Rad50 is physically associated with human Mre11: identification of a conserved multiprotein complex implicated in recombinational DNA repair. Mol Cell Biol, 16, 4832-4841.

Dumaz, N. and Meek, D.W. (1999) Serine15 phosphorylation stimulates p53 
transactivation but does not directly influence interaction with HDM2. Embo $J, 18,7002-$ 7010 .

Durocher, D., Henckel, J., Fersht, A.R. and Jackson, S.P. (1999) The FHA domain is a modular phosphopeptide recognition motif. Mol Cell, 4, 387-394.

Dutertre, S., Sekhri, R., Tintignac, L.A., Onclercq-Delic, R., Chatton, B., Jaulin, C. and Amor-Gueret, M. (2002) Dephosphorylation and subcellular compartment change of the mitotic Bloom's syndrome DNA helicase in response to ionizing radiation. $J$ Biol Chem, 277, 6280-6286.

Eder, E., Kutt, W. and Deininger, C. (2001) On the role of alkylating mechanisms, Oalkylation and DNA-repair in genotoxicity and mutagenicity of alkylating methanesulfonates of widely varying structures in bacterial systems. Chem Biol Interact, 137, 89-99.

Edwards, R.J., Bentley, N.J. and Carr, A.M. (1999) A Rad3-Rad26 complex responds to DNA damage independently of other checkpoint proteins. Nat Cell Biol, 1, 393-398.

Ekholm, S.V. and Reed, S.I. (2000) Regulation of G(1) cyclin-dependent kinases in the mammalian cell cycle. Curr Opin Cell Biol, 12, 676-684.

Emili, A. (1998) MEC1-dependent phosphorylation of Rad9p in response to DNA damage. Mol Cell, 2, 183-189.

Emili, A., Schieltz, D.M., Yates, J.R., 3rd and Hartwell, L.H. (2001) Dynamic interaction of DNA damage checkpoint protein Rad53 with chromatin assembly factor Asf1. Mol Cell, 7, 13-20.

Enoch, T., Carr, A.M. and Nurse, P. (1992) Fission yeast genes involved in coupling mitosis to completion of DNA replication. Genes Dev, 6, 2035-2046.

Esashi, F. and Yanagida, M. (1999) Cdc2 phosphorylation of Crb2 is required for reestablishing cell cycle progression after the damage checkpoint. Mol Cell, 4, 167-174.

Falck, J., Mailand, N., Syljuasen, R.G., Bartek, J. and Lukas, J. (2001) The ATM-Chk2Cdc25A checkpoint pathway guards against radioresistant DNA synthesis. Nature, $\mathbf{4 1 0}$, 842-847.

Fasullo, M., Bennett, T., AhChing, P. and Koudelik, J. (1998) The Saccharomyces cerevisiae RAD9 checkpoint reduces the DNA damage-associated stimulation of directed translocations. Mol Cell Biol, 18, 1190-1200.

Fitz Gerald, J.N., Benjamin, J.M. and Kron, S.J. (2002) Robust Gl checkpoint arrest in budding yeast: dependence on DNA damage signaling and repair. $J$ Cell Sci, 115, 1749- 
1757.

Foiani, M., Pellicioli, A., Lopes, M., Lucca, C., Ferrari, M., Liberi, G., Muzi Falconi, M. and Plevani, P. (2000) DNA damage checkpoints and DNA replication controls in Saccharomyces cerevisiae. Mutat Res, 451, 187-196.

Forrest, A. and Gabrielli, B. (2001) Cdc25B activity is regulated by 14-3-3. Oncogene, 20, 4393-4401.

Foss, E.J. (2001) Toflp regulates DNA damage responses during $\mathrm{S}$ phase in Saccharomyces cerevisiae. Genetics, 157, 567-577.

Frei, C. and Gasser, S.M. (2000) The yeast Sgslp helicase acts upstream of Rad53p in the DNA replication checkpoint and colocalizes with Rad53p in S-phase-specific foci. Genes Dev, 14, 81-96.

Frei, C. and Gasser, S.M. (2000) RecQ-like helicases: the DNA replication checkpoint connection. J Cell Sci, 113 (15), 2641-2646.

Gardner, R., Putnam, C.W. and Weinert, T. (1999) RAD53, DUN1 and PDS1 define two parallel G2/M checkpoint pathways in budding yeast. Embo J, 18, 3173-3185.

Gartner, A., Milstein, S., Ahmed, S., Hodgkin, J. and Hengartner, M.O. (2000) A conserved checkpoint pathway mediates DNA damage--induced apoptosis and cell cycle arrest in C. elegans. Mol Cell, 5, 435-443.

Garvik, B., Carson, M. and Hartwell, L. (1995) Single-stranded DNA arising at telomeres in cdc 13 mutants may constitute a specific signal for the RAD9 checkpoint. Mol Cell Biol, 15, 6128-6138.

Gasch, A.P., Huang, M., Metzner, S., Botstein, D., Elledge, S.J. and Brown, P.O. (2001) Genomic expression responses to DNA-damaging agents and the regulatory role of the yeast ATR homolog Mec1p. Mol Biol Cell, 12, 2987-3003.

Gatei, M., Scott, S.P., Filippovitch, I., Soronika, N., Lavin, M.F., Weber, B. and Khanna, K.K. (2000) Role for ATM in DNA damage-induced phosphorylation of BRCA1. Cancer Res, 60, 3299-3304.

Gately, D.P., Hittle, J.C., Chan, G.K. and Yen, T.J. (1998) Characterization of ATM expression, localization, and associated DNA-dependent protein kinase activity. Mol Biol Cell, 9, 2361-2374.

Gayther, S.A., Pharoah, P.D. and Ponder, B.A. (1998) The genetics of inherited breast cancer. J Mammary Gland Biol Neoplasia, 3, 365-376. 
Geske, F.J., Nelson, A.C., Lieberman, R., Strange, R., Sun, T. and Gerschenson, L.E. (2000) DNA repair is activated in early stages of p53-induced apoptosis. Cell Death Differ, 7, 393-401.

Geyer, R.K., Nagasawa, H., Little, J.B. and Maki, C.G. (2000) Role and regulation of p53 during an ultraviolet radiation-induced G1 cell cycle arrest. Cell Growth Differ, 11, 149156.

Gilbert, C.S., Green, C.M. and Lowndes, N.F. (2001) Budding yeast Rad9 is an ATPdependent Rad53 activating machine. Mol Cell, 8, 129-136.

Gowen, L.C., Avrutskaya, A.V., Latour, A.M., Koller, B.H. and Leadon, S.A. (1998) BRCA1 required for transcription-coupled repair of oxidative DNA damage. Science, 281, 1009-1012.

Grandin, N., Reed, S.I. and Charbonneau, M. (1997) Stn1, a new Saccharomyces cerevisiae protein, is implicated in telomere size regulation in association with Cdc13. Genes Dev, 11, 512-527.

Graves, P.R., Lovly, C.M., Uy, G.L. and Piwnica-Worms, H. (2001) Localization of human $\mathrm{Cdc} 25 \mathrm{C}$ is regulated both by nuclear export and 14-3-3 protein binding. Oncogene, 20, 1839-1851.

Green, C.M., Erdjument-Bromage, H., Tempst, P. and Lowndes, N.F. (2000) A novel Rad24 checkpoint protein complex closely related to replication factor C. Curr Biol, 10, 39-42.

Grenon, M., Tillit, J., Piard, K., Baldacci, G. and Francesconi, S. (1999) The S/M checkpoint at 37 degrees $\mathrm{C}$ and the recovery of viability of the mutant poldeltats 3 require the crb2+/rhp9+ gene in fission yeast. Mol Gen Genet, 260, 522-534.

Griffiths, D.J., Barbet, N.C., McCready, S., Lehmann, A.R. and Carr, A.M. (1995) Fission yeast rad 17: a homologue of budding yeast RAD24 that shares regions of sequence similarity with DNA polymerase accessory proteins. Embo J, 14, 5812-5823.

Grushcow, J.M., Holzen, T.M., Park, K.J., Weinert, T., Lichten, M. and Bishop, D.K. (1999) Saccharomyces cerevisiae checkpoint genes MEC1, RAD17 and RAD24 are required for normal meiotic recombination partner choice. Genetics, 153, 607-620.

Guo, Z., Kumagai, A., Wang, S.X. and Dunphy, W.G. (2000) Requirement for Atr in phosphorylation of Chkl and cell cycle regulation in response to DNA replication blocks and UV-damaged DNA in Xenopus egg extracts. Genes Dev, 14, 2745-2756. 
Hallet, B., Sherratt, D.J. and Hayes, F. (1997) Pentapeptide scanning mutagenesis: random insertion of a variable five amino acid cassette in a target protein. Nucleic Acids Res, 25, 1866-1867.

Hang, H. and Lieberman, H.B. (2000) Physical interactions among human checkpoint control proteins HUS1p, RAD1p, and RAD9p, and implications for the regulation of cell cycle progression. Genomics, 65, 24-33.

Harper, J.W., Adami, G.R., Wei, N., Keyomarsi, K. and Elledge, S.J. (1993) The p21 Cdk-interacting protein $\mathrm{Cipl}$ is a potent inhibitor of $\mathrm{Gl}$ cyclin-dependent kinases. Cell, 75, 805-816.

Hartwell, L.H. and Weinert, T.A. (1989) Checkpoints: controls that ensure the order of cell cycle events. Science, 246, 629-634.

Haupt, Y., Maya, R., Kazaz, A. and Oren, M. (1997) Mdm2 promotes the rapid degradation of p53. Nature, 387, 296-299.

Hayes, F., Hallet, B. and Cao, Y. (1997) Insertion mutagenesis as a tool in the modification of protein function. Extended substrate specificity conferred by pentapeptide insertions in the omega-loop of TEM-1 beta-lactamase. J Biol Chem, 272, 28833-28836.

Hekmat-Nejad, M., You, Z., Yee, M.C., Newport, J.W. and Cimprich, K.A. (2000) Xenopus ATR is a replication-dependent chromatin-binding protein required for the DNA replication checkpoint. Curr Biol, 10, 1565-1573.

Hermeking, H., Lengauer, C., Polyak, K., He, T.C., Zhang, L., Thiagalingam, S., Kinzler, K.W. and Vogelstein, B. (1997) 14-3-3 sigma is a p53-regulated inhibitor of G2/M progression. Mol Cell, 1, 3-11.

Hirao, A., Kong, Y.Y., Matsuoka, S., Wakeham, A., Ruland, J., Yoshida, H., Liu, D., Elledge, S.J. and Mak, T.W. (2000) DNA damage-induced activation of p53 by the checkpoint kinase Chk2. Science, 287, 1824-1827.

Hofmann, K. and Bucher, P. (1995) The FHA domain: a putative nuclear signalling domain found in protein kinases and transcription factors. Trends Biochem Sci, 20, 347 349.

Hong, E.J. and Roeder, G.S. (2002) A role for Ddc1 in signaling meiotic double-strand breaks at the pachytene checkpoint. Genes Dev, 16, 363-376.

Hu, F., Alcasabas, A.A. and Elledge, S.J. (2001) Asfl links Rad53 to control of chromatin assembly. Genes Dev, 15, 1061-1066. 
Huyton, T., Bates, P.A., Zhang, X., Sternberg, M.J. and Freemont, P.S. (2000) The BRCA1 C-terminal domain: structure and function. Mutat Res, 460, 319-332.

Iftode, C., Daniely, Y. and Borowiec, J.A. (1999) Replication protein A (RPA): the eukaryotic SSB. Crit Rev Biochem Mol Biol, 34, 141-180.

Ji, X., Zhang, P., Armstrong, R.N. and Gilliland, G.L. (1992) The three-dimensional structure of a glutathione S-transferase from the mu gene class. Structural analysis of the binary complex of isoenzyme 3-3 and glutathione at 2.2-A resolution. Biochemistry, 31, 10169-10184.

Jin, P., Hardy, S. and Morgan, D.O. (1998) Nuclear localization of cyclin B1 controls mitotic entry after DNA damage. J Cell Biol, 141, 875-885.

Kamijo, T., van de Kamp, E., Chong, M.J., Zindy, F., Diehl, J.A., Sherr, C.J. and McKinnon, P.J. (1999) Loss of the ARF tumor suppressor reverses premature replicative arrest but not radiation hypersensitivity arising from disabled atm function. Cancer Res, 59, 2464-2469.

Kang, D., Chen, J., Wong, J. and Fang, G. (2002) The checkpoint protein Chfr is a ligase that ubiquitinates Plk1 and inhibits $\mathrm{Cdc} 2$ at the G2 to M transition. J Cell Biol, 156, 249259.

Kastan, M.B., Onyekwere, O., Sidransky, D., Vogelstein, B. and Craig, R.W. (1991) Participation of p53 protein in the cellular response to DNA damage. Cancer Res, $\mathbf{5 1}$, 6304-6311.

Kastan, M.B. and Lim, D.S. (2000) The many substrates and functions of ATM. Nat Rev Mol Cell Biol, 1, 179-186.

Kawabe, T., Suganuma, M., Ando, T., Kimura, M., Hori, H. and Okamoto, T. (2002)

$\mathrm{Cdc} 25 \mathrm{C}$ interacts with PCNA at G2/M transition. Oncogene, 21, 1717-1726.

Khanna, K.K. and Jackson, S.P. (2001) DNA double-strand breaks: signaling, repair and the cancer connection. Nat Genet, 27, 247-254.

Khosravi, R., Maya, R., Gottlieb, T., Oren, M., Shiloh, Y. and Shkedy, D. (1999) Rapid ATM-dependent phosphorylation of MDM2 precedes $\mathrm{p} 53$ accumulation in response to DNA damage. Proc Natl Acad Sci U S A, 96, 14973-14977.

Kim, H.S. and Brill, S.J. (2001) Rfc4 interacts with Rpal and is required for both DNA replication and DNA damage checkpoints in Saccharomyces cerevisiae. Mol Cell Biol, 21, 3725-3737. 
Klein, H.L. (2001) Spontaneous chromosome loss in Saccharomyces cerevisiae is suppressed by DNA damage checkpoint functions. Genetics, 159, 1501-1509.

Kohn, P.H., Whang-Peng, J. and Levis, W.R. (1982) Chromosomal instability in ataxia telangiectasia. Cancer Genet Cytogenet, 6, 289-302.

Komatsu, K., Miyashita, T., Hang, H., Hopkins, K.M., Zheng, W., Cuddeback, S., Yamada, M., Lieberman, H.B. and Wang, H.G. (2000) Human homologue of S. pombe Rad9 interacts with BCL-2/BCL-xL and promotes apoptosis. Nat Cell Biol, 2, 1-6.

Kondo, T., Wakayama, T., Naiki, T., Matsumoto, K. and Sugimoto, K. (2001)

Recruitment of Mecl and Ddcl checkpoint proteins to double-strand breaks through distinct mechanisms. Science, 294, 867-870.

Koonin, E.V., Altschul, S.F. and Bork, P. (1996) BRCAl protein products ... Functional motifs. Nat Genet, 13, 266-268.

Ladbury, J.E., Lemmon, M.A., Zhou, M., Green, J., Botfield, M.C. and Schlessinger, J. (1995) Measurement of the binding of tyrosyl phosphopeptides to SH2 domains: a reappraisal. Proc Natl Acad Sci U S A, 92, 3199-3203.

Lavin, M.F. and Shiloh, Y. (1997) The genetic defect in ataxia-telangiectasia. Annu Rev Immunol, 15, 177-202.

Lee, J.S., Collins, K.M., Brown, A.L., Lee, C.H. and Chung, J.H. (2000) hCds1-mediated phosphorylation of BRCA1 regulates the DNA damage response. Nature, 404, 201-204.

Lee, J., Kumagai, A. and Dunphy, W.G. (2001) Positive regulation of Weel by Chk1 and 14-3-3 proteins. Mol Biol Cell, 12, 551-563.

Leu, J.Y. and Roeder, G.S. (1999) The pachytene checkpoint in S. cerevisiae depends on Swel-mediated phosphorylation of the cyclin-dependent kinase Cdc28. Mol Cell, 4, 805814.

Li, J., Smith, G.P. and Walker, J.C. (1999) Kinase interaction domain of kinaseassociated protein phosphatase, a phosphoprotein-binding domain. Proc Natl Acad Sci U $S A, 96,7821-7826$.

Li, S., Ting, N.S., Zheng, L., Chen, P.L., Ziv, Y., Shiloh, Y., Lee, E.Y. and Lee, W.H. (2000) Functional link of BRCA1 and ataxia telangiectasia gene product in DNA damage response. Nature, 406, 210-215.

Liao, H., Byeon, I.J. and Tsai, M.D. (1999) Structure and function of a new 
phosphopeptide-binding domain containing the FHA2 of Rad53. J Mol Biol, 294, 10411049.

Liao, H., Yuan, C., Su, M.I., Yongkiettrakul, S., Qin, D., Li, H., Byeon, I.J., Pei, D. and Tsai, M.D. (2000) Structure of the FHA1 domain of yeast Rad53 and identification of binding sites for both FHAl and its target protein Rad9. J Mol Biol, 304, 941-951.

Lim, D.S., Kim, S.T., Xu, B., Maser, R.S., Lin, J., Petrini, J.H. and Kastan, M.B. (2000) ATM phosphorylates p95/nbs1 in an S-phase checkpoint pathway. Nature, 404, 613-617.

Lindsay, H.D., Griffiths, D.J., Edwards, R.J., Christensen, P.U., Murray, J.M., Osman, F., Walworth, N. and Carr, A.M. (1998) S-phase-specific activation of Cds 1 kinase defines a subpathway of the checkpoint response in Schizosaccharomyces pombe. Genes Dev, 12 , 382-395.

Lindsey-Boltz, L.A., Bermudez, V.P., Hurwitz, J. and Sancar, A. (2001) Purification and characterization of human DNA damage checkpoint Rad complexes. Proc Natl Acad Sci $U S A, 98,11236-11241$.

Liu, F., Rothblum-Oviatt, C., Ryan, C.E. and Piwnica-Worms, H. (1999) Overproduction of human Myt1 kinase induces a G2 cell cycle delay by interfering with the intracellular trafficking of Cdc2-cyclin B1 complexes. Mol Cell Biol, 19, 5113-5123.

Loeb, L.A. (1991) Mutator phenotype may be required for multistage carcinogenesis. Cancer Res, 51, 3075-3079.

Longhese, M.P., Neecke, H., Paciotti, V., Lucchini, G. and Plevani, P. (1996) The 70 kDa subunit of replication protein $A$ is required for the G1/S and intra-S DNA damage checkpoints in budding yeast. Nucleic Acids Res, 24, 3533-3537.

Longhese, M.P., Paciotti, V., Neecke, H. and Lucchini, G. (2000) Checkpoint proteins influence telomeric silencing and length maintenance in budding yeast. Genetics, 155, 1577-1591.

Longtine, M.S., McKenzie, A., 3rd, Demarini, D.J., Shah, N.G., Wach, A., Brachat, A., Philippsen, P. and Pringle, J.R. (1998) Additional modules for versatile and economical PCR-based gene deletion and modification in Saccharomyces cerevisiae. Yeast, 14, 953961.

Lopes, M., Cotta-Ramusino, C., Pellicioli, A., Liberi, G., Plevani, P., Muzi-Falconi, M., Newlon, C.S. and Foiani, M. (2001) The DNA replication checkpoint response stabilizes stalled replication forks. Nature, 412, 557-561.

Lopez-Girona, A., Kanoh, J. and Russell, P. (2001) Nuclear exclusion of Cdc25 is not 
required for the DNA damage checkpoint in fission yeast. Curr Biol, 11, 50-54.

Lydall, D. and Weinert, T. (1995) Yeast checkpoint genes in DNA damage processing: implications for repair and arrest. Science, 270, 1488-1491.

Lydall, D., Nikolsky, Y., Bishop, D. K., and Weinert, T. (1996) A meiotic recombination checkpoint controlled by mitotic checkpoint genes. Nature, 383, 840-843.

Mailand, N., Falck, J., Lukas, C., Syljuasen, R.G., Welcker, M., Bartek, J. and Lukas, J. (2000) Rapid destruction of human Cdc25A in response to DNA damage. Science, 288, 1425-1429.

Makiniemi, M., Hillukkala, T., Tuusa, J., Reini, K., Vaara, M., Huang, D., Pospiech, H., Majuri, I., Westerling, T., Makela, T.P. and Syvaoja, J.E. (2001) BRCT domaincontaining protein TopBP1 functions in DNA replication and damage response. $J$ Biol Chem, 276, 30399-30406.

Malkin, D., Li, F.P., Strong, L.C., Fraumeni, J.F., Jr., Nelson, C.E., Kim, D.H., Kassel, J., Gryka, M.A., Bischoff, F.Z., Tainsky, M.A. and et al. (1990) Germ line p53 mutations in a familial syndrome of breast cancer, sarcomas, and other neoplasms. Science, 250, 1233 1238.

Maringele, L. and Lydall, D. (2002) EXO1-dependent single-stranded DNA at telomeres activates subsets of DNA damage and spindle checkpoint pathways in budding yeast yku70Delta mutants. Genes Dev, 16, 1919-1933.

Martinho, R.G., Lindsay, H.D., Flaggs, G., DeMaggio, A.J., Hoekstra, M.F., Carr, A.M. and Bentley, N.J. (1998) Analysis of Rad3 and Chk1 protein kinases defines different checkpoint responses. Embo J, 17, 7239-7249.

Maru, Y., Afar, D.E., Witte, O.N. and Shibuya, M. (1996) The dimerization property of glutathione S-transferase partially reactivates Bcr-Abl lacking the oligomerization domain. J Biol Chem, 271, 15353-15357.

Maru, Y. (2000) Use of glutathione S-transferase and break point cluster region protein as artificial dimerization domains to activate tyrosine kinases. Methods Enzymol, 327, 429-440.

Maser, R.S., Monsen, K.J., Nelms, B.E. and Petrini, J.H. (1997) hMre 11 and hRad50 nuclear foci are induced during the normal cellular response to DNA double-strand breaks. Mol Cell Biol, 17, 6087-6096.

Masson, M., Niedergang, C., Schreiber, V., Muller, S., Menissier-de Murcia, J. and de Murcia, G. (1998) XRCC1 is specifically associated with poly(ADP-ribose) polymerase 
and negatively regulates its activity following DNA damage. Mol Cell Biol, 18, 35633571 .

Matsuoka, S., Huang, M. and Elledge, S.J. (1998) Linkage of ATM to cell cycle regulation by the Chk2 protein kinase. Science, 282, 1893-1897.

Matsuura, A., Naito, T. and Ishikawa, F. (1999) Genetic control of telomere integrity in Schizosaccharomyces pombe: $\operatorname{rad} 3(+)$ and tell $(+)$ are parts of two regulatory networks independent of the downstream protein kinases chkl(+) and cdsl( + . Genetics, 152, 1501-1512.

McAinsh, A.D., Scott-Drew, S., Murray, J.A. and Jackson, S.P. (1999) DNA damage triggers disruption of telomeric silencing and Meclp-dependent relocation of Sir3p. Curr Biol, 9, 963-966.

Melo, J.A., Cohen, J. and Toczyski, D.P. (2001) Two checkpoint complexes are independently recruited to sites of DNA damage in vivo. Genes Dev, 15, 2809-2821.

Mills, K.D., Sinclair, D.A. and Guarente, L. (1999) MEC1-dependent redistribution of the Sir3 silencing protein from telomeres to DNA double-strand breaks. Cell, 97, 609620.

Morrison, C., Sonoda, E., Takao, N., Shinohara, A., Yamamoto, K. and Takeda, S. (2000) The controlling role of ATM in homologous recombinational repair of DNA damage. Embo J, 19, 463-471.

Moynahan, M.E., Chiu, J.W., Koller, B.H. and Jasin, M. (1999) Brcal controls homology-directed DNA repair. Mol Cell, 4, 511-518.

Naiki, T., Shimomura, T., Kondo, T., Matsumoto, K. and Sugimoto, K. (2000) Rfc5, in cooperation with rad24, controls DNA damage checkpoints throughout the cell cycle in Saccharomyces cerevisiae. Mol Cell Biol, 20, 5888-5896.

Nash, R.A., Caldecott, K.W., Barnes, D.E. and Lindahl, T. (1997) XRCC1 protein interacts with one of two distinct forms of DNA ligase III. Biochemistry, 36, 5207-5211.

Navas, T.A., Sanchez, Y. and Elledge, S.J. (1996) RAD9 and DNA polymerase epsilon form parallel sensory branches for transducing the DNA damage checkpoint signal in Saccharomyces cerevisiae. Genes Dev, 10, 2632-2643.

Noskov, V.N., Araki, H. and Sugino, A. (1998) The RFC2 gene, encoding the thirdlargest subunit of the replication factor $\mathrm{C}$ complex, is required for an S-phase checkpoint in Saccharomyces cerevisiae. Mol Cell Biol, 18, 4914-4923. 
Nugent, C.I., Hughes, T.R., Lue, N.F. and Lundblad, V. (1996) Cdc13p: a single-strand telomeric DNA-binding protein with a dual role in yeast telomere maintenance. Science, 274, 249-252.

Oakley, G.G., Loberg, L.I., Yao, J., Risinger, M.A., Yunker, R.L., Zernik-Kobak, M., Khanna, K.K., Lavin, M.F., Carty, M.P. and Dixon, K. (2001) UV-induced hyperphosphorylation of replication protein a depends on DNA replication and expression of ATM protein. Mol Biol Cell, 12, 1199-1213.

Okumura, E., Fukuhara, T., Yoshida, H., Hanada Si, S., Kozutsumi, R., Mori, M., Tachibana, K. and Kishimoto, T. (2002) Akt inhibits Mytl in the signalling pathway that leads to meiotic G2/M-phase transition. Nat Cell Biol, 4, 111-116.

Paciotti, V., Clerici, M., Lucchini, G. and Longhese, M.P. (2000) The checkpoint protein $\mathrm{Ddc} 2$, functionally related to $\mathrm{S}$. pombe Rad26, interacts with $\mathrm{Mec1}$ and is regulated by Mec1-dependent phosphorylation in budding yeast. Genes Dev, 14, 2046-2059.

Paciotti, V., Clerici, M., Scotti, M., Lucchini, G. and Longhese, M.P. (2001) Characterization of mec1 kinase-deficient mutants and of new hypomorphic mec1 alleles impairing subsets of the DNA damage response pathway. Mol Cell Biol, 21, 3913-3925.

Pandita, T.K. (2002) ATM function and telomere stability. Oncogene, 21, 611-618.

Park, H. and Sternglanz, R. (1999) Identification and characterization of the genes for two topoisomerase I-interacting proteins from Saccharomyces cerevisiae. Yeast, 15, 3541.

Parker, M.W., Lo Bello, M. and Federici, G. (1990) Crystallization of glutathione Stransferase from human placenta. J Mol Biol, 213, 221-222.

Parker, A.E., Van de Weyer, I., Laus, M.C., Oostveen, I., Yon, J., Verhasselt, P. and Luyten, W.H. (1998) A human homologue of the Schizosaccharomyces pombe rad1+ checkpoint gene encodes an exonuclease. J Biol Chem, 273, 18332-18339.

Paull, T.T., Cortez, D., Bowers, B., Elledge, S.J. and Gellert, M. (2001) From the Cover: Direct DNA binding by Brcal. Proc Natl Acad Sci U S A, 98, 6086-6091.

Paulovich, A.G. and Hartwell, L.H. (1995) A checkpoint regulates the rate of progression through S phase in S. cerevisiae in response to DNA damage. Cell, 82, 841-847.

Paulovich, A.G., Toczyski, D.P. and Hartwell, L.H. (1997) When checkpoints fail. Cell, 88, 315-321.

Paulovich, A.G., Armour, C.D. and Hartwell, L.H. (1998) The Saccharomyces cerevisiae 
RAD9, RAD17, RAD24 and MEC3 genes are required for tolerating irreparable, ultraviolet-induced DNA damage. Genetics, 150, 75-93.

Pellicioli, A., Lee, S.E., Lucca, C., Foiani, M. and Haber, J.E. (2001) Regulation of Saccharomyces Rad53 checkpoint kinase during adaptation from DNA damage-induced G2/M arrest. Mol Cell, 7, 293-300.

Peng, C.Y., Graves, P.R., Thoma, R.S., Wu, Z., Shaw, A.S. and Piwnica-Worms, H. (1997) Mitotic and G2 checkpoint control: regulation of 14-3-3 protein binding by phosphorylation of Cdc25C on serine-216. Science, 277, 1501-1505.

Perrault, R., Cheong, N., Wang, H. and Iliakis, G. (2001) RPA facilitates rejoining of DNA double-strand breaks in an in vitro assay utilizing genomic DNA as substrate. Int $J$ Radiat Biol, 77, 593-607.

Raghuraman, M.K., Winzeler, E.A., Collingwood, D., Hunt, S., Wodicka, L., Conway, A., Lockhart, D.J., Davis, R.W., Brewer, B.J. and Fangman, W.L. (2001) Replication dynamics of the yeast genome. Science, 294, 115-121.

Ren, B., Cam, H., Takahashi, Y., Volkert, T., Terragni, J., Young, R.A. and Dynlacht, B.D. (2002) E2F integrates cell cycle progression with DNA repair, replication, and G(2)/M checkpoints. Genes Dev, 16, 245-256.

Rhind, N. and Russell, P. (2001) Roles of the mitotic inhibitors Weel and Mik1 in the G(2) DNA damage and replication checkpoints. Mol Cell Biol, 21, 1499-1508.

Riley, L.G., Ralston, G.B. and Weiss, A.S. (1996) Multimer formation as a consequence of separate homodimerization domains: the human c-Jun leucine zipper is a transplantable dimerization module. Protein Eng, 9, 223-230.

Ritchie, K.B., Mallory, J.C. and Petes, T.D. (1999) Interactions of TLC1 (which encodes the RNA subunit of telomerase), TEL1, and MEC1 in regulating telomere length in the yeast Saccharomyces cerevisiae. Mol Cell Biol, 19, 6065-6075.

Rotman, G. and Shiloh, Y. (1998) ATM: from gene to function. Hum Mol Genet, 7, 1555-1563.

Rotman, G. and Shiloh, Y. (1999) ATM: a mediator of multiple responses to genotoxic stress. Oncogene, 18, 6135-6144.

Rouse, J. and Jackson, S.P. (2000) LCD1: an essential gene involved in checkpoint control and regulation of the MEC1 signalling pathway in Saccharomyces cerevisiae. Embo J, 19, 5801-5812. 
Saka, Y., Esashi, F., Matsusaka, T., Mochida, S. and Yanagida, M. (1997) Damage and replication checkpoint control in fission yeast is ensured by interactions of $\mathrm{Crb} 2$, a protein with BRCT motif, with Cut5 and Chk1. Genes Dev, 11, 3387-3400.

Sanchez, Y., Wong, C., Thoma, R.S., Richman, R., Wu, Z., Piwnica-Worms, H. and Elledge, S.J. (1997) Conservation of the Chkl checkpoint pathway in mammals: linkage of DNA damage to Cdk regulation through Cdc25. Science, 277, 1497-1501.

Sanchez, Y., Bachant, J., Wang, H., Hu, F., Liu, D., Tetzlaff, M. and Elledge, S.J. (1999) Control of the DNA damage checkpoint by chkl and rad53 protein kinases through distinct mechanisms. Science, 286, 1166-1171.

Sandell, L.L. and Zakian, V.A. (1993) Loss of a yeast telomere: arrest, recovery, and chromosome loss. Cell, 75, 729-739.

Santocanale, C., Neecke, H., Longhese, M.P., Lucchini, G. and Plevani, P. (1995) Mutations in the gene encoding the $34 \mathrm{kDa}$ subunit of yeast replication protein A cause defective S phase progression. J Mol Biol, 254, 595-607.

Santocanale, C. and Diffley, J.F. (1998) A Mecl- and Rad53-dependent checkpoint controls late-firing origins of DNA replication. Nature, 395, 615-618.

Savitsky, K., Bar-Shira, A., Gilad, S., Rotman, G., Ziv, Y., Vanagaite, L., Tagle, D.A., Smith, S., Uziel, T., Sfez, S. and et al. (1995) A single ataxia telangiectasia gene with a product similar to PI-3 kinase. Science, 268, 1749-1753.

Schramke, V., Neecke, H., Brevet, V., Corda, Y., Lucchini, G., Longhese, M.P., Gilson, E. and Geli, V. (2001) The set1Delta mutation unveils a novel signaling pathway relayed by the Rad53-dependent hyperphosphorylation of replication protein A that leads to transcriptional activation of repair genes. Genes Dev, 15, 1845-1858.

Schwartz, M.F., Duong, J.K., Sun, Z., Morrow, J.S., Pradhan, D. and Stern, D.F. (2002) Rad9 phosphorylation sites couple Rad53 to the Saccharomyces cerevisiae DNA damage checkpoint. Mol Cell, 9, 1055-1065.

Scolnick, D.M. and Halazonetis, T.D. (2000) Chfr defines a mitotic stress checkpoint that delays entry into metaphase. Nature, 406, 430-435.

Scully, R., Chen, J., Plug, A., Xiao, Y., Weaver, D., Feunteun, J., Ashley, T. and Livingston, D.M. (1997) Association of BRCA1 with Rad51 in mitotic and meiotic cells. Cell, 88, 265-275.

Scully, R. and Livingston, D.M. (2000) In search of the tumour-suppressor functions of BRCA1 and BRCA2. Nature, 408, 429-432. 
Sherr, C.J. and Roberts, J.M. (1999) CDK inhibitors: positive and negative regulators of G1-phase progression. Genes Dev, 13, 1501-1512.

Shieh, S.Y., Ahn, J., Tamai, K., Taya, Y. and Prives, C. (2000) The human homologs of checkpoint kinases Chk1 and Cds1 (Chk2) phosphorylate p53 at multiple DNA damageinducible sites. Genes Dev, 14, 289-300.

Shiloh, Y. (1997) Ataxia-telangiectasia and the Nijmegen breakage syndrome: related disorders but genes apart. Annu Rev Genet, 31, 635-662.

Shiloh, Y. (2001) ATM and ATR: networking cellular responses to DNA damage. Curr Opin Genet Dev, 11, 71-77.

Shimada, M., Okuzaki, D., Tanaka, S., Tougan, T., Tamai, K.K., Shimoda, C. and Nojima, H. (1999) Replication factor C3 of Schizosaccharomyces pombe, a small subunit of replication factor $\mathrm{C}$ complex, plays a role in both replication and damage checkpoints. Mol Biol Cell, 10, 3991-4003.

Shortle, D., Novick, P. and Botstein, D. (1984) Construction and genetic characterization of temperature-sensitive mutant alleles of the yeast actin gene. Proc Natl Acad Sci US A, 81, 4889-4893.

Sidorova, J.M. and Breeden, L.L. (1997) Rad53-dependent phosphorylation of Swi6 and down-regulation of CLN1 and CLN2 transcription occur in response to DNA damage in Saccharomyces cerevisiae. Genes Dev, 11, 3032-3045.

Siede, W., Friedberg, A.S., Dianova, I. and Friedberg, E.C. (1994) Characterization of G1 checkpoint control in the yeast Saccharomyces cerevisiae following exposure to DNAdamaging agents. Genetics, 138, 271-281.

Smith, G.C. and Jackson, S.P. (1999) The DNA-dependent protein kinase. Genes Dev, 13, 916-934.

Smith, G.C., Cary, R.B., Lakin, N.D., Hann, B.C., Teo, S.H., Chen, D.J. and Jackson, S.P. (1999) Purification and DNA binding properties of the ataxia-telangiectasia gene product ATM. Proc Natl Acad Sci US A, 96, 11134-11139.

Smits, V.A., Klompmaker, R., Arnaud, L., Rijksen, G., Nigg, E.A. and Medema, R.H. (2000) Polo-like kinase-1 is a target of the DNA damage checkpoint. Nat Cell Biol, 2, 672-676.

Snouwaert, J.N., Gowen, L.C., Latour, A.M., Mohn, A.R., Xiao, A., DiBiase, L. and Koller, B.H. (1999) BRCA1 deficient embryonic stem cells display a decreased 
homologous recombination frequency and an increased frequency of non-homologous recombination that is corrected by expression of a brcal transgene. Oncogene, 18, 79007907.

Soulier, J. and Lowndes, N.F. (1999) The BRCT domain of the S. cerevisiae checkpoint protein Rad9 mediates a Rad9-Rad9 interaction after DNA damage. Curr Biol, 9, 551554.

St Onge, R.P., Udell, C.M., Casselman, R. and Davey, S. (1999) The human G2 checkpoint control protein hRAD9 is a nuclear phosphoprotein that forms complexes with hRAD1 and hHUS1. Mol Biol Cell, 10, 1985-1995.

Stewart, G.S., Maser, R.S., Stankovic, T., Bressan, D.A., Kaplan, M.I., Jaspers, N.G., Raams, A., Byrd, P.J., Petrini, J.H. and Taylor, A.M. (1999) The DNA double-strand break repair gene hMRE11 is mutated in individuals with an ataxia-telangiectasia-like disorder. Cell, 99, 577-587.

Sugawara, N. and Haber, J.E. (1992) Characterization of double-strand break-induced recombination: homology requirements and single-stranded DNA formation. Mol Cell Biol, 12, 563-575.

Sun, Z., Hsiao, J., Fay, D.S. and Stern, D.F. (1998) Rad53 FHA domain associated with phosphorylated Rad9 in the DNA damage checkpoint. Science, 281, 272-274.

Takekawa, M., Adachi, M., Nakahata, A., Nakayama, I., Itoh, F., Tsukuda, H., Taya, Y. and Imai, K. (2000) p53-inducible wipl phosphatase mediates a negative feedback regulation of p38 MAPK-p53 signaling in response to UV radiation. Embo J, 19, 65176526.

Tanaka, H., Arakawa, H., Yamaguchi, T., Shiraishi, K., Fukuda, S., Matsui, K., Takei, Y. and Nakamura, Y. (2000) A ribonucleotide reductase gene involved in a p53-dependent cell-cycle checkpoint for DNA damage. Nature, 404, 42-49.

Tanaka, K. and Russell, P. (2001) Mrcl channels the DNA replication arrest signal to checkpoint kinase Cds1. Nat Cell Biol, 3, 966-972.

Taylor, R.M., Wickstead, B., Cronin, S. and Caldecott, K.W. (1998) Role of a BRCT domain in the interaction of DNA ligase III-alpha with the DNA repair protein XRCC1. Curr Biol, 8, 877-880.

Taylor, W.R., DePrimo, S.E., Agarwal, A., Agarwal, M.L., Schonthal, A.H., Katula, K.S. and Stark, G.R. (1999) Mechanisms of G2 arrest in response to overexpression of p53. Mol Biol Cell, 10, 3607-3622. 
Taylor, W.R. and Stark, G.R. (2001) Regulation of the G2/M transition by p53.

Oncogene, 20, 1803-1815.

Taylor, R.M., Thistlethwaite, A. and Caldecott, K.W. (2002) Central role for the XRCC1 BRCT I domain in mammalian DNA single-strand break repair. Mol Cell Biol, 22, 25562563.

Terada, Y., Tatsuka, M., Jinno, S. and Okayama, H. (1995) Requirement for tyrosine phosphorylation of Cdk4 in G1 arrest induced by ultraviolet irradiation. Nature, 376, 358-362.

Tercero, J.A. and Diffley, J.F. (2001) Regulation of DNA replication fork progression through damaged DNA by the Mec1/Rad53 checkpoint. Nature, 412, 553-557.

Thompson, D.A. and Stahl, F.W. (1999) Genetic control of recombination partner preference in yeast meiosis. Isolation and characterization of mutants elevated for meiotic unequal sister-chromatid recombination. Genetics, 153, 621-641.

Tibbetts, R.S., Cortez, D., Brumbaugh, K.M., Scully, R., Livingston, D., Elledge, S.J. and Abraham, R.T. (2000) Functional interactions between BRCA1 and the checkpoint kinase ATR during genotoxic stress. Genes Dev, 14, 2989-3002.

Tinker-Kulberg, R.L. and Morgan, D.O. (1999) Pds 1 and Espl control both anaphase and mitotic exit in normal cells and after DNA damage. Genes Dev, 13, 1936-1949.

Toyoshima, F., Moriguchi, T., Wada, A., Fukuda, M. and Nishida, E. (1998) Nuclear export of cyclin B1 and its possible role in the DNA damage-induced G2 checkpoint. Embo J, 17, 2728-2735.

Tsurimoto, T. and Stillman, B. (1991) Replication factors required for SV40 DNA replication in vitro. II. Switching of DNA polymerase alpha and delta during initiation of leading and lagging strand synthesis. J Biol Chem, 266, 1961-1968.

Tsuzuki, T., Fujii, Y., Sakumi, K., Tominaga, Y., Nakao, K., Sekiguchi, M., Matsushiro, A., Yoshimura, Y. and MoritaT. (1996) Targeted disruption of the Rad51 gene leads to lethality in embryonic mice. Proc Natl Acad Sci U S A, 93, 6236-6240.

Tyler, J.K., Adams, C.R., Chen, S.R., Kobayashi, R., Kamakaka, R.T. and Kadonaga, J.T. (1999) The RCAF complex mediates chromatin assembly during DNA replication and repair. Nature, 402, 555-560.

Usui, T., Ogawa, H. and Petrini, J.H. (2001) A DNA damage response pathway controlled by Tel1 and the Mre11 complex. Mol Cell, 7, 1255-1266. 
van Brabant, A.J., Buchanan, C.D., Charboneau, E., Fangman, W.L. and Brewer, B.J. (2001) An origin-deficient yeast artificial chromosome triggers a cell cycle checkpoint. Mol Cell, 7, 705-713.

van Vugt, M.A., Smits, V.A., Klompmaker, R. and Medema, R.H. (2001) Inhibition of Polo-like kinase-1 by DNA damage occurs in an ATM- or ATR-dependent fashion. $J$ Biol Chem, 276, 41656-41660.

Venclovas, C. and Thelen, M.P. (2000) Structure-based predictions of Rad1, Rad9, Hus1 and Rad 17 participation in sliding clamp and clamp-loading complexes. Nucleic Acids Res, 28, 2481-2493.

Verkade, H.M. and O'Connell, M.J. (1998) Cut5 is a component of the UV-responsive DNA damage checkpoint in fission yeast. Mol Gen Genet, 260, 426-433.

Vialard, J.E., Gilbert, C.S., Green, C.M. and Lowndes, N.F. (1998) The budding yeast Rad9 checkpoint protein is subjected to Mec 1/Tell-dependent hyperphosphorylation and interacts with Rad53 after DNA damage. Embo J, 17, 5679-5688.

Volkmer, E. and Karnitz, L.M. (1999) Human homologs of Schizosaccharomyces pombe rad1, hus 1, and rad9 form a DNA damage-responsive protein complex. J Biol Chem, 274, 567-570.

Wakayama, T., Kondo, T., Ando, S., Matsumoto, K. and Sugimoto, K. (2001) Pie1, a protein interacting with $\mathrm{Mec} 1$, controls cell growth and checkpoint responses in Saccharomyces cerevisiae. Mol Cell Biol, 21, 755-764.

Walker, J., Crowley, P., Moreman, A.D. and Barrett, J. (1993) Biochemical properties of cloned glutathione S-transferases from Schistosoma mansoni and Schistosoma japonicum. Mol Biochem Parasitol, 61, 255-264.

Wang, H. and Elledge, S.J. (1999) DRC1, DNA replication and checkpoint protein 1, functions with DPB 11 to control DNA replication and the S-phase checkpoint in Saccharomyces cerevisiae. Proc Natl Acad Sci U S A, 96, 3824-3829.

Wang, Y., Cortez, D., Yazdi, P., Neff, N., Elledge, S.J. and Qin, J. (2000) BASC, a super complex of BRCA1-associated proteins involved in the recognition and repair of aberrant DNA structures. Genes Dev, 14, 927-939.

Wang, P., Byeon, I.J., Liao, H., Beebe, K.D., Yongkiettrakul, S., Pei, D. and Tsai, M.D. (2000) II. Structure and specificity of the interaction between the FHA2 domain of Rad53 and phosphotyrosyl peptides. $J$ Mol Biol, 302, 927-940.

Wang, H., Zeng, Z.C., Bui, T.A., DiBiase, S.J., Qin, W., Xia, F., Powell, S.N. and Iliakis, 
G. (2001) Nonhomologous end-joining of ionizing radiation-induced DNA doublestranded breaks in human tumor cells deficient in BRCA1 or BRCA2. Cancer Res, 61, 270-277.

Wang, H., Guan, J., Perrault, A.R., Wang, Y. and Iliakis, G. (2001) Replication protein A2 phosphorylation after DNA damage by the coordinated action of ataxia telangiectasiamutated and DNA-dependent protein kinase. Cancer Res, 61, 8554-8563.

Ward, J.F. (1985) Biochemistry of DNA lesions. Radiat Res Suppl, 8, S103-111.

Ward, I.M. and Chen, J. (2001) Histone H2AX is phosphorylated in an ATR-dependent manner in response to replicational stress. J Biol Chem, 276, 47759-47762.

Weinert, T.A. and Hartwell, L.H. (1988) The RAD9 gene controls the cell cycle response to DNA damage in Saccharomyces cerevisiae. Science, 241, 317-322.

Weinert, T.A. and Hartwell, L.H. (1990) Characterization of RAD9 of Saccharomyces cerevisiae and evidence that its function acts posttranslationally in cell cycle arrest after DNA damage. Mol Cell Biol, 10, 6554-6564.

Weinert, T.A., Kiser, G.L. and Hartwell, L.H. (1994) Mitotic checkpoint genes in budding yeast and the dependence of mitosis on DNA replication and repair. Genes Dev, 8, 652-665.

Weinert, T. (1997) Yeast checkpoint controls and relevance to cancer. Cancer Surv, 29, 109-132.

Weinert, T. (1998) DNA damage checkpoints update: getting molecular. Curr Opin Genet Dev, 8, 185-193.

Weinreich, M. and Stillman, B. (1999) Cdc7p-Dbf4p kinase binds to chromatin during S phase and is regulated by both the APC and the RAD53 checkpoint pathway. Embo J, 18, 5334-5346.

Willson, J., Wilson, S., Warr, N. and Watts, F.Z. (1997) Isolation and characterization of the Schizosaccharomyces pombe rhp9 gene: a gene required for the DNA damage checkpoint but not the replication checkpoint. Nucleic Acids Res, 25, 2138-2146.

Wolkow, T.D. and Enoch, T. (2002) Fission yeast rad26 is a regulatory subunit of the rad3 checkpoint kinase. Mol Biol Cell, 13, 480-492.

Wright, J.A., Keegan, K.S., Herendeen, D.R., Bentley, N.J., Carr, A.M., Hoekstra, M.F. and Concannon, P. (1998) Protein kinase mutants of human ATR increase sensitivity to UV and ionizing radiation and abrogate cell cycle checkpoint control. Proc Natl Acad Sci 
$U S A, 95,7445-7450$.

Wu, J.R. and Gilbert, D.M. (1995) Rapid DNA preparation for 2D gel analysis of replication intermediates. Nucleic Acids Res, 23, 3997-3998.

Xie, S., Wu, H., Wang, Q., Cogswell, J.P., Husain, I., Conn, C., Stambrook, P., JhanwarUniyal, M. and Dai, W. (2001) Plk3 functionally links DNA damage to cell cycle arrest and apoptosis at least in part via the p53 pathway. J Biol Chem, 276, 43305-43312.

Xiong, Y., Zhang, H. and Beach, D. (1992) D type cyclins associate with multiple protein kinases and the DNA replication and repair factor PCNA. Cell, 71, 505-514.

$\mathrm{Xu}, \mathrm{Y}$. and Baltimore, D. (1996) Dual roles of ATM in the cellular response to radiation and in cell growth control. Genes Dev, 10, 2401-2410.

Xu, B., Kim, S. and Kastan, M.B. (2001) Involvement of Brcal in S-phase and G(2)phase checkpoints after ionizing irradiation. Mol Cell Biol, 21, 3445-3450.

Yamane, K. and Tsuruo, T. (1999) Conserved BRCT regions of TopBP1 and of the tumor suppressor BRCA1 bind strand breaks and termini of DNA. Oncogene, 18, 5194-5203.

Yamane, K., Katayama, E. and Tsuruo, T. (2000) The BRCT regions of tumor suppressor BRCA1 and of XRCCl show DNA end binding activity with a multimerizing feature. Biochem Biophys Res Commun, 279, 678-684.

Yamane, K., Wu, X. and Chen, J. (2002) A DNA damage-regulated BRCT-containing protein, TopBP1, is required for cell survival. Mol Cell Biol, 22, 555-566.

Yamazaki, V., Wegner, R.D. and Kirchgessner, C.U. (1998) Characterization of cell cycle checkpoint responses after ionizing radiation in Nijmegen breakage syndrome cells. Cancer Res, 58, 2316-2322.

Yang, S.S., Yeh, E., Salmon, E.D. and Bloom, K. (1997) Identification of a mid-anaphase checkpoint in budding yeast. $J$ Cell Biol, 136, 345-354.

Yarden, R.I. and Brody, L.C. (1999) BRCA1 interacts with components of the histone deacetylase complex. Proc Natl Acad Sci U S A, 96, 4983-4988.

Yarden, R.I., Pardo-Reoyo, S., Sgagias, M., Cowan, K.H. and Brody, L.C. (2002)

BRCA1 regulates the $\mathrm{G} 2 / \mathrm{M}$ checkpoint by activating Chk1 kinase upon DNA damage. Nat Genet, 30, 285-289.

You, Z., Kong, L. and Newport, J. (2002) The role of single-stranded DNA and Pol a in establishing the ATR, Hus1 DNA replication checkpoint. J Biol Chem, 277, 27088-93. 
Zhang, X., Morera, S., Bates, P.A., Whitehead, P.C., Coffer, A.I., Hainbucher, K., Nash, R.A., Sternberg, M.J., Lindahl, T. and Freemont, P.S. (1998) Structure of an XRCC1 BRCT domain: a new protein-protein interaction module. Embo J, 17, 6404-6411.

Zhao, X., Muller, E.G. and Rothstein, R. (1998) A suppressor of two essential checkpoint genes identifies a novel protein that negatively affects dNTP pools. Mol Cell, 2, 329-340.

Zhao, S., Weng, Y.C., Yuan, S.S., Lin, Y.T., Hsu, H.C., Lin, S.C., Gerbino, E., Song, M.H., Zdzienicka, M.Z., Gatti, R.A., Shay, J.W., Ziv, Y., Shiloh, Y. and Lee, E.Y. (2000) Functional link between ataxia-telangiectasia and Nijmegen breakage syndrome gene products. Nature, 405, 473-477.

Zhao, X., Chabes, A., Domkin, V., Thelander, L. and Rothstein, R. (2001) The ribonucleotide reductase inhibitor Smll is a new target of the Mec1/Rad53 kinase cascade during growth and in response to DNA damage. Embo J, 20, 3544-3553.

Zhao, X. and Rothstein, R. (2002) The Dun1 checkpoint kinase phosphorylates and regulates the ribonucleotide reductase inhibitor Sml1. Proc Natl Acad Sci USA, 99, 3746-3751.

Zhong, Q., Chen, C.F., Li, S., Chen, Y., Wang, C.C., Xiao, J., Chen, P.L., Sharp, Z.D. and Lee, W.H. (1999) Association of BRCA1 with the hRad50-hMre11-p95 complex and the DNA damage response. Science, 285, 747-750.

Zhou, B.B., Chaturvedi, P., Spring, K., Scott, S.P., Johanson, R.A., Mishra, R., Mattern, M.R., Winkler, J.D. and Khanna, K.K. (2000) Caffeine abolishes the mammalian G(2)/M DNA damage checkpoint by inhibiting ataxia-telangiectasia-mutated kinase activity. $J$ Biol Chem, 275, 10342-10348.

Zhu, X.D., Kuster, B., Mann, M., Petrini, J.H. and Lange, T. (2000) Cell-cycle-regulated association of RAD50/MRE11/NBS1 with TRF2 and human telomeres. Nat Genet, 25, 347-352.

Zou, L., Cortez, D. and Elledge, S.J. (2002) Regulation of ATR substrate selection by Rad17-dependent loading of Rad9 complexes onto chromatin. Genes Dev, 16, 198-208. 